\title{
The Evolution of Cluster Elliptical Galaxies
}

by

Luis Felipe Barrientos

A Thesis submitted in conformity with the requirements for the degree of Doctor of Philosophy Graduate Department of Astronomy University of Toronto

(C) Copyright by Luis Felipe Barrientos 1999 
Acquisitions and Bibliographic Services

395 Wellington Street Ottawa ON K1A ON4 Canada

\section{du Canada}

Acquisitions et services bibliographiques

395, rue Wellington

Ottawa ON K1A ON4

Canada
The author has granted a nonexclusive licence allowing the National Library of Canada to reproduce, loan, distribute or sell copies of this thesis in microform, paper or electronic formats.

The author retains ownership of the copyright in this thesis. Neither the thesis nor substantial extracts from it may be printed or otherwise reproduced without the author's permission.
L'auteur a accordé une licence non exclusive permettant à la Bibliothèque nationale du Canada de reproduire, prêter, distribuer ou vendre des copies de cette thèse sous la forme de microfiche/film, de reproduction sur papier ou sur format électronique.

L'auteur conserve la propriété du droit d'auteur qui protège cette thèse. $\mathrm{Ni}$ la thèse ni des extraits substantiels de celle-ci ne doivent être imprimés ou autrement reproduits sans son autorisation.

\section{Canadä}




\title{
The Evolution of Cluster Elliptical Galaxies
}

\author{
Luis Felipe Barrientos \\ Doctor of Philosophy, 1999 \\ Department of Astronomy, University of Toronto
}

\begin{abstract}
This thesis work focuses on the understanding of the formation and subsequent evolution of the population of elliptical galaxies in rich galaxy clusters. Elliptical galaxies define a unique and simple laboratory to study the process of galaxy formation and the evolution of stellar populations. Observational data, including a sample of eight clusters observed at Cerro Tololo Inter-American Observatory (CTIO) and ten clusters observed with the Hubble Space Telescope (HST), have been used to conduct several related studies.

A surface brightness analysis of the ellipticals in the cores of ten galaxy clusters observed with HST shows that elliptical galaxies are well-represented by an $r^{1 / 4}$-law light profile and follow a well-defined relation in the magnitude/size plane, up to $z=$ 1.2. This relation shifts, on average, towards higher luminosities (or equivalently, to smaller sizes) with increasing redshift. If we assume that the size of these galaxies are fixed during the period from $z=1.2$ to the present, then these galaxies are evolving monotonically in luminosity. This luminosity evolution amounts to $\Delta M_{B}=-1.2$ at $z=1.2$, when compared to the galaxies in local clusters, and is consistent with that expected for an old and passively evolving stellar population.

The luminosity functions for the red galaxies from the eight clusters in the CTIO sample show that are well-represented by a Schechter function, consistent with a single value for their characteristic magnitude $\left(\mathrm{M}^{*}\right)$. When compared to local cluster luminosity functions, there is evidence for a brightening of a few tenths of a magnitude in $M^{*}$ (the exact value depends on the cosmology). For a low density universe, the brightening observed in the luminosity function, up to $z=0.45$, is consistent with that observed from the surface brightness analysis.
\end{abstract}


The cluster elliptical galaxies from the CTIO sample, selected morphologically using a galaxy light profile fitting technique, define a very tight sequence in the colour/magnitude plane. All the clusters are consistent with having a single value for their intrinsic colour dispersions, $\delta=0.06 \pm 0.02$, in the restframe $(U-V) / V$ colour relation. These small colour dispersions imply the stars in these galaxies have a typical age dispersion from galaxy to galaxy of $\sim 20 \%$ of their age at $z=0.45$. Similarly, when comparing the mean colour for the different clusters, it was found that they are consistent with having a single mean colour, $(V-I)=2.055 \pm 0.028$. The small dispersion in the mean colours is completely accounted for by sampling uncertainties.

With the addition of $K$-band photometry, and data for local ellipticals, it has been possible to determine the evolutionary vector in the restframe $(U-V) /(V-H)$ colour plane from $z=0.45$ to $z=0$. Predictions of current models have been tested against this observed vector. Most of the more popular models have failed to account for this colour change, and only a more sophisticated model -including infall of primordial gas and chemical enrichment during the assembly of the stellar population- was able to reproduce the observed evolution.

The observations in this work strongly suggest that these galaxies are old systems formed at high redshift. However, given the quantitative uncertainties in the observations, population synthesis models, cosmology, and uncertainties in how much synchronicity in formation is reasonable, the observations do not rule out scenarios involving formation at lower redshifts. 


\section{A mi familia}




\section{Acknowledgements}

Many people have contributed and helped me in the successful completion of this thesis, I am grateful to all of them. In particular, I want to thank:

Daniela and Javier for their constant support, love and understanding.

Simon Lilly for his invaluable advice, judgment, comments, ideas and financial support.

David Schade for his friendship, ideas, software and computing resources. All the galaxy fits were performed by David at DAO.

Bob Garrison for helping me to come to Toronto. I appreciate his help, particularly during my first year in Toronto.

My friends at the Department of Astronomy, especially Omar López-Cruz, Marcin Sawicki, Tracy Clarke, James Wadsley, James Brown and Gabriela Mallén-Ornelas for their constant support and numerous lunches together.

Lillian Lanca and Toni Young for their help and good sense of humor.

And finally, to all my friends in the astronomy department and in the Flamengo and Pagaea soccer teams. 


\section{Contents}

1 Introduction 1

1.1 Background .......................... 1

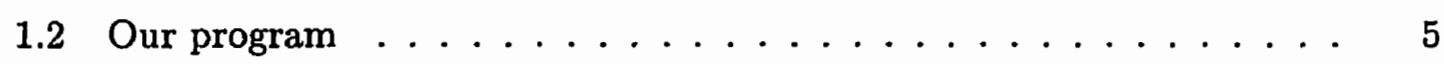

1.2 .1 Aims ......................... 5

1.2 .2 Observational approach . .................. 7

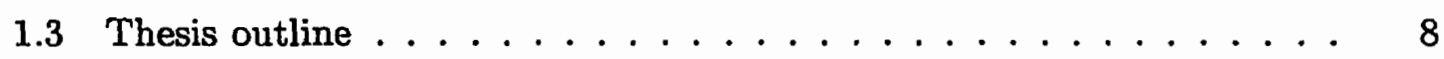

2 Observations $\quad 10$

2.1 Sample selection . . . . . . . . . . . . . . 10

2.2 Optical data . . . . . . . . . . . . . . . . . . 12

2.2 .1 Observing strategy ............................. 13

2.2.2 Generation of superflats .............. 15

2.2 .3 Image distortions . . . . . . . . . . . . . 17

2.2.4 Photometric calibration ................. 17

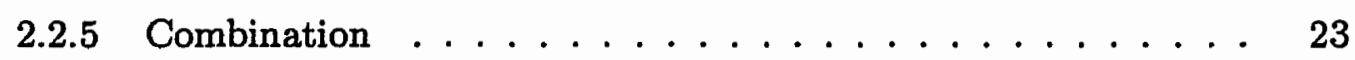

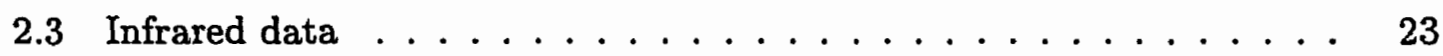

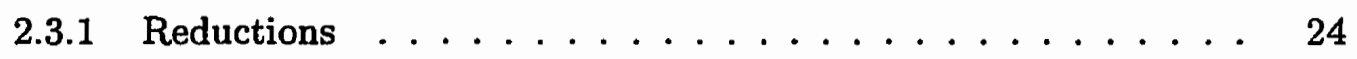

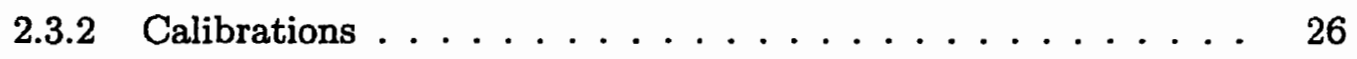

2.4 Photometric catalogs . . . . . . . . . . . . . 27

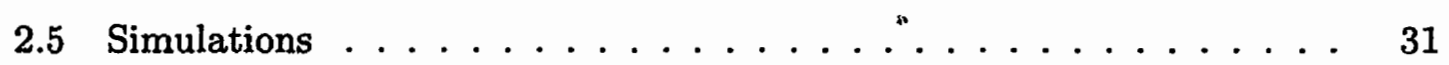

2.6 Cluster images $\ldots \ldots \ldots \ldots \ldots \ldots \ldots \ldots$ 
3 Evolution in surface brightness: study of clusters using HST 43

3.1 Observations ..................... 43

3.2 Local calibration ..................... 46

3.3 Results . . . . . . . . . . . . . . . . . 48

3.4 Subsequent confirmation of this result . . . . . . . . . . 51

3.5 Summary .............................. 51

4 Evolution in the luminosity function from the CTIO sample 53

4.1 Background correction .................... 54

4.1.1 Field galaxy counts . . . . . . . . . . . . . 56

4.2 Count distributions of red cluster galaxies . . . . . . . . . 57

4.3 Fitting procedure ........................... 59

4.4 Results ............................ 63

4.5 Constraints to $q_{0} \ldots \ldots \ldots \ldots$

4.6 Summary ............................ 70

5 Colour Dispersions $\quad 71$

5.1 Sample selection . . . . . . . . . . . . . . . 72

5.1.1 Spectroscopic redshift sample ............... 72

5.1 .2 Morphological sample . . . . . . . . . . . 76

5.1.3 Colour sample.................. 80

5.2 Dispersion within clusters ..................... 83

5.2.1 Measurement of colours .............. 84

5.2.2 Estimate of dispersion and mean colour . . . . . . . . 85

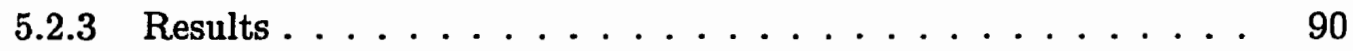

5.3 Dispersion from cluster to cluster ............... 91

5.3.1 Corrections to the mean colours ............. 91

5.3.2 Estimate of dispersion between clusters . . . . . . . . . 93

5.4 Discussion - light-weighted ages . . . . . . . . . . 95

5.5 Interpretation . . . . . . . . . . . . . 98

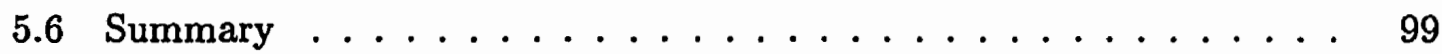


6 Colour Evolution $\quad 101$

6.1 Galaxy colours at $z=0.45 \ldots \ldots . \ldots 10 . \ldots \ldots$

6.2 Local ellipticals . . . . . . . . . . . . . . . . . . . 104

6.3 Observed colour evolution ................. 105

6.4 Spectral synthesis population models . . . . . . . . . . 107

6.5 Ages for ellipticals . . . . . . . . . . . . . 116

6.5.1 Consequence of assuming very early formation . . . . . . . . 117

6.5 .2 Consequence of adopting low $\Omega \ldots \ldots \ldots$

6.6 Summary . . . . . . . . . . . . . . . . 120

7 Summary and Conclusions $\quad 121$

7.1 Summary . . . . . . . . . . . . . . . . 121

7.2 Evolutionary history of cluster ellipticals . . . . . . . . . . 122

7.3 Bottom line . . . . . . . . . . . . . . . . 124

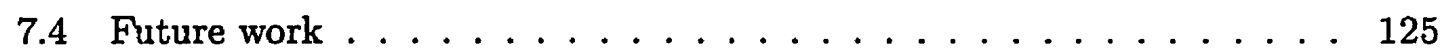

$\begin{array}{lr}\text { A Catalogs } & 127\end{array}$ 


\section{List of Figures}

2.1 Transmition curves for the $V$ and $I$ filters . . . . . . . . . . . . 14

2.2 Exposure delay image for the optical images . . . . . . . . . . 18

2.3 Standard stars calibration for March $1996 \ldots \ldots 21$

2.4 Standard stars calibration for December $1996 \ldots \ldots 22$

2.5 Transmition curve for the $K$-band filter . . . . . . . . . . . . 24

2.6 Stellarity index measured by sextractor . . . . . . . . . . . . 29

2.7 Colour-colour diagram for the objects in the cluster . . . . . . . 30

2.8 Simulations to obtain the detection rate and estimate the photometric accuracy of sextractor in the field of Cluster $4 \ldots \ldots 32$

2.9 Simulations to obtain the detection rate and estimate the photometric accuracy of sextractor in the field of Cluster $15 \ldots \ldots 33$

$2.10 \mathrm{a} I$-band image of the cluster J1275.15TR . . . . . . . . . . 35

2.10bI-band image of the cluster J1556.15BL . . . . . . . . . . 36

$2.10 \mathrm{c} I$-band image of the cluster F1652.20CR . . . . . . . . . 37

$2.10 \mathrm{~d} I$-band image of the cluster $\mathrm{J} 2001.21 \mathrm{C} \ldots \ldots . \ldots 38$

$2.10 \mathrm{e} I$-band image of the cluster J1834.8BL $\ldots \ldots \ldots$

2.10f $I$-band image of the cluster F1835.22CR . . . . . . . . . . . . 40

$2.10 \mathrm{~g} I$-band image of the cluster $\mathrm{RXJ1347.5-11.45} \ldots \ldots$. . . . . . . 41

2.10hI-band image of the cluster F1637.23TL . . . . . . . . . . . 42

3.1 Relation between $M_{A B}(B)$ and $\log R_{e}$ for elliptical galaxies in moderate and high-redshift clusters . . . . . . . . . . . . . 47 
3.2 Luminosity shift $\Delta M_{B}$ for the HST clusters with respect to the local

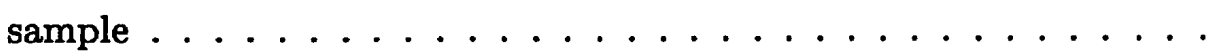

4.1 Field correction for the clusters in the CTIO sample . . . . . . . . . . 55

4.2 Composite colour-magnitude diagram for all the galaxies in the cluster fields . . . . . . . . . . . . . . . . . .

4.3 Luminosity functions for the red galaxies in the clusters of the CTIO sample . . . . . . . . . . . . . . . . .

4.4 Combination of the observed luminosity functions for all the clusters

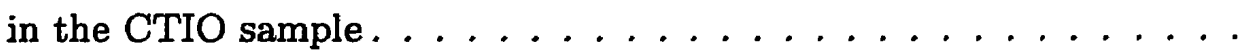

4.5 Best fit parameters for the Schechter functions for the luminosity functions of the CTIO sample . . . . . . . . . . . . . .

4.6 $I^{*}$ value from the Schechter function fits as a function of the redshift of the cluster .......................... 65

4.7 Cosmology constraint from the luminosity evolution . . . . . . . . 69

5.1 Optical colour-magnitude diagram for all the galaxies confirmed as cluster members.

5.2 Infrared colour-magnitude diagram for all the galaxies confirmed as cluster members.

5.3 Example of the procedure to classify morphologically the galaxies in these clusters ...................... . 79

5.4 Expected colour-colour tracks for galaxies with different SEDs . . . 81

5.5 Infrared colour-magnitude diagram for all the elliptical galaxies in the fields of the eight clusters of the sample . . . . . . . . . . . . . . . . 82

5.6 Optical colour-magnitude diagram for the elliptical galaxies in the central $1 \mathrm{Mpc}$ of the cluster fields . . . . . . . . . . . . 87

5.7 Colour corrections . . . . . . . . . . . . . . . . 92

5.8 Mean $(V-I)$ colour and intrinsic dispersion for the galaxies in each of the clusters of the sample . . . . . . . . . . . . . . . 94

5.9 Colour evolution of a simple stellar population . . . . . . . . . . 96 
$6.1(V-H) /(U-V)$ colour evolution predicted for different models . . . 106

$6.2(H-K)$ colour evolution of a simple stellar system predicted by dif-

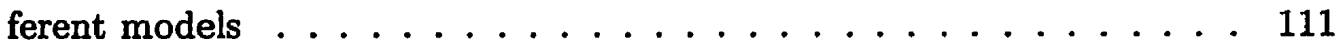

6.3 Colour evolution for a solar metallicity galaxy . . . . . . . . . 113

6.4 Colour evolution for a low metallicity galaxy . . . . . . . . . . . . 114

6.5 Colour evolution for a high metallicity galaxy $\ldots \ldots . . . \ldots 115$

6.6 Constraint on the ages of these galaxies using the absolute colours . . 119 


\section{List of Tables}

2.1 Cluster sample observed in this thesis work . . . . . . . . . . . . 12

2.2 Details of the optical observations for the clusters in this work . . . 13

2.3 Summary of the transformation coefficients for the IR data . . . . . 26

2.4 Transformation coefficients used in the IR corrections. . . . . . . . . 27

3.1 Clusters selected from the HST archive . . . . . . . . . . . . 44

3.2 Subsequent determinations of luminosity evolution for elliptical galaxies 51

4.1 Fits to the field counts. . . . . . . . . . . . . . 56

4.2 $I^{*}$ and $N^{*}$ values for the best Schechter function fit for the clusters of the CTIO sample .................... 63

5.1 Observed cluster mean colour and colour dispersions . . . . . . . 88

5.2 Mean cluster $(V-I)$ colour and intrinsic colour dispersion for the elliptical galaxies for the clusters in the sample . . . . . . . . . . 93

6.1 Summary of the restframe colours for the E/S0 galaxies in this study 105

6.2 Colour evolution of elliptical galaxies from the literature and this work 107

6.3 Summary of input parameters for the evolutionary models in this study 109

6.4 Elapsed time between $z=0.45$ and $z=0.00$ and age of the universe for different cosmological models . . . . . . . . . . . . . . 112

6.5 Predicted $(U-V)$ colour evolution from the models . . . . . . . . 117 


\section{Chapter 1}

\section{Introduction}

\subsection{Background}

Present day galaxies show a wide range of morphologies, colours and properties. Behind the present appearance, each galaxy has a history which depends on the conditions of its formation and the environment where it has developed. The understanding of the galaxy formation process and the subsequent evolution of galaxies over a significant fraction of the age of the universe are two of the main goals in astronomy today.

Noticeable evolutionary changes in galaxies generally have a timescale much longer than a human life time (or of astronomical observations) and therefore it is not possible to follow the evolution of individual objects. This requires a different approach to study the galaxy evolution. Characterizing field galaxies at high redshift through redshift surveys is one way of setting constraints for the evolution of galaxies. These redshift surveys, however, share the same problem that most of the evolutionary studies suffer. Since it is not easy to relate high redshift objects to the local ones. It means that only conclusions about the whole population, or subsamples, of galaxies can be drawn. For instance, these field surveys have detected a change in the luminosity function of the general galaxy population up to $z \sim 1$ (e.g., Lilly et al. 1995).

Complementary to field surveys are the cluster surveys. This approach has the 
advantages of: 1) being much cheaper in telescope time due to the large number of galaxies concentrated in a small volume, 2) having all the galaxies essentially the same redshift, and 3) hosting mostly ellipticals, which are the simplest stellar systems on galactic scales. In addition, elliptical galaxies in high redshift clusters can be easily linked to ellipticals in local clusters, i.e. it is possible to track the evolution of this subset of the general galaxy population because clusters are longlived entities. On the other hand, using cluster galaxies for studying the evolution of galaxies requires careful consideration of the following: 1) just a small fraction of the population of galaxies in the universe is in clusters, 2) although clusters are longlived entities, the number may be changing as new clusters are formed, so clusters at high redshift may not be representative progenitors of present-day clusters, and 3) the environmental conditions in clusters are extreme when compared to the field conditions. Therefore, it is reasonable to expect important differences between the histories of field galaxies and those in clusters. As an example, the ratio between Ellipticals/S0/Spirals (E:S0:Sp) varies from 10:20:70 to 20:40:40 between the field and a cluster (Oemler 1992), respectively. Early-type galaxies dominate the local cluster population.

E/SO galaxies at the present epoch form a very homogeneous population. Bower et al. (1992a) performed high precision $U V J K$ photometry for 94 early-type galaxies in the Coma and Virgo galaxy clusters. They found a small intrinsic scatter of about $0.04 \mathrm{mag}$ in the $(U-V) / V$ colour-magnitude relation for the $\mathrm{E} / \mathrm{S} 0$ galaxies in Coma. This scatter vanishes when the S0 galaxies are excluded from the sample. They also found that there is no significant difference between the colour-magnitude relation for early-type galaxies observed in Coma and the same relation observed in Virgo, supporting the universality of the colour-magnitude relation in clusters. This is quite remarkable because Coma and Virgo have very different morphologies, richnesses, galaxy content, central concentrations, subclustering, etc. which would suggests that these do not influence the colours of the E/S0 population. Similar work by LópezCruz (1997) found a well defined red sequence in $(B-R) / R$, with small observed scatter $\left(\sigma_{(B-R)} \sim 0.1 \mathrm{mag}\right)$, in the central regions of 45 Abell clusters. 
Besides their colours, ellipticals are also homogeneous in other respects. Djorgovski and Davis (1987) and Dressler et al. (1987), independently and almost simultaneously, found that elliptical galaxies follow extremely tight relations between their observables: velocity dispersion, mean central surface brightness and effective radius. These quantities define the fundamental plane whose small thickness (i.e. dispersion) can be completely accounted by observational errors.

Recent photometric studies of cluster galaxies at intermediate and high redshifts (Lilly 1987; Aragón-Salamanca et al. 1993; Rakos and Schombert 1995; Stanford et al. 1995; Ellis et al. 1997; Standford et al. 1998) have shown that E/S0 galaxies have bluer colours than their counterparts at lower redshifts. The observed evolution in colour of the red envelope is broadly consistent with current models that include the effects of passive evolution, i.e. the dimming of the constituent stars after an initial single burst of star formation at a given earlier epoch. Aragón-Salamanca et al. (1993) used the change in colour and spectral synthesis model predictions to set the initial galaxy formation time for these galaxies in the range $2<z<5$. The small difference in time ( $~ 1-3 \mathrm{Gyr}$, depending on the cosmology) for the galaxy formation process implies some coordination in the formation of galaxies. Rakos and Schombert (1995), on the other hand, used different photometry and models and found that the mean epoch of galaxy formation is at $z \sim 5$, but the reddest objects require formation at $z \sim 10$. Using infrared and optical photometry (with $\lambda>4000 \AA$ at restframe) Stanford et al. (1995) found that the colour-magnitude relation for two moderate redshifts clusters at $(z \sim 0.4)$ is similar to that in Coma, supporting the idea of a similar time for the galaxy formation. Following the same philosophy as Aragón-Salamanca et al. (1993), Standford et al. (1998) used better instrumentation - particularly in the IR-, to show that the red sequence is present up to $z=0.9$ in their sample of 19 galaxy clusters. Their observed dispersion in the colour-magnitude relation is consistent with having a single low value all through the sample. This small dispersion has also been detected at high/moderate redshifts by Ellis et al. (1997) through HST observations of three rich clusters at $z=0.54$.

The studies presented above refer to galaxy formation in the context of their 
constituent stellar populations and not necessarily to the times or process by which material was assembled in galaxies. In this respect, there are two main views for the formation of giant elliptical galaxies: 1) a protogalactic monolithic collapse with dissipational star formation, and 2) a product of mergers in a hierarchical scenario of structure formation. The first view for the formation of galaxies explains naturally the colour-magnitude relation and its small dispersion as the product of a metallicity difference between the more massive and luminous galaxies, with respect to those less massive and fainter. Galactic winds would occur progressively later in more massive galaxies allowing for a longer period of metal enrichment of their stellar populations, and thus producing slightly redder colours (Arimoto and Yoshii 1987; Bressan et al. 1994; Tantalo et al. 1996; Kodama and Arimoto 1997). Hierarchical models of structure formation, on the other hand, produce massive galaxies by combining smaller units over an extended period (Baugh et al. 1996), making these massive galaxies relatively recent events. In spite of their relatively short assembly ages, these models can reproduce the tightness of the colour-magnitude relation (Kauffmann 1996) and the slope of the relation as a metallicity difference (Kauffmann and Charlot 1998). This requires that most of the stellar content in the final galaxy has already been formed in the smaller units and thus the light-weighted age corresponds to that of the stars in these smaller units. In this scenario, more massive ellipticals are made out of more massive fragments. In this model the homogeneity of this galaxy population at high redshift is understood as a bias effect. Clusters observed at high redshift are rare and massive, and therefore they have "turned around" (collapsed) much earlier than local clusters. While the colour-magnitude relation and its small intrinsic dispersion is a natural consequence of single monolithic collapse models, hierarchical models are less readily consistent with the small dispersion but can be made so. To resolve between these two possibilities, it is necessary to study a large complete sample of clusters at high redshift, and/or study the field ellipticals that should not be affected by extreme conditions of galaxy clusters.

While most of the galaxies in the universe show traces of recent star formation diluting the signature of the formation process of the bulk of their stars, early-type 
galaxies, ellipticals in particular, show no sign of recent star formation. This fact, together with the evidence presented above distinguish the population of $\mathrm{E} / \mathrm{S} 0 \mathrm{~s}$ in galaxy clusters as a simple and unique laboratory to study galaxy formation and evolution. This makes them the natural stepping-stone into galactic scales from the well-studied globular clusters in the Galaxy.

Spectral population synthesis models summarize most of our knowledge of stellar evolution, through the use of evolutionary tracks, stellar atmospheres, the initial mass function, etc. These models are currently being widely used to draw conclusions about the universe, and therefore, problems in the models would certainly have an impact on our perception of the universe. For example, some techniques to determine $q_{0}$ depend on the exact knowledge of the luminosity evolution that "standard candles" have experienced at cosmological distances; and some photometric redshift techniques compare the colours of the galaxies to those of the models to estimate the galaxy redshifts. These spectral synthesis models have to be, therefore, thoroughly tested to validate any conclusions drawn by their use. It is possible to obtain colours of elliptical galaxies in clusters over a wide baseline in time. These colours provide a test for these spectral synthesis models. At this stage, colours of ellipticals at different times in their evolution seem to be well represented by simple stellar population synthesis models. However, high quality data is required in order to carefully test these models.

\subsection{Our program}

\subsubsection{Aims}

Motivated by the results of the high precision photometry work of Bower et al. (1992a) for the galaxies in the Coma and Virgo clusters, we decided to extend this work to higher redshifts. The smail intrinsic dispersion in the colour-magnitude relation they find implies that the ratio $(\delta t / t)$ is small, where $\delta t$ is the light-weighted age spread for the formation of the galaxies, and $t$ is the typical light-weighted age of these galaxies. The small $(\delta t / t)$ measured for the $\mathrm{E} / \mathrm{S} 0$ galaxies in Coma implies 
a very synchronized process of formation, and/or an old age for these objects. By extending the work to higher redshifts, we can study these galaxies when they are at a much younger age than their equivalent galaxies in local clusters, and thus increase our sensitivity of the measurement of the $(\delta t / t)$ ratio. The dispersion in the mean colours for several clusters, similarly to the dispersion in the colour-magnitude for the galaxies within each cluster, provides information about the dispersion in the mean galaxy formation epoch in the clusters.

Luminosity evolution is a natural consequence of an aging stellar population that it believed to make up the elliptical galaxies in clusters. The integrated light in these galaxies is dominated by the stars on their main sequence and in their giant branch. Luminosity evolution depends strongly on the initial mass function as it is governed by the change in the absolute number of stars on the main sequence and in the giant branch. Therefore, determining the evolution in luminosity helps us to constrain the initial mass function in elliptical galaxies.

Colour evolution for these galaxies is relatively mild and depends mainly on the colour change of the turnoff point. As the stellar population gets older, the brighter and bluer stars are removed from the main sequence, thus decreasing the effective temperature of the turnoff point. The number of stars in the giant branch corresponds approximately to the number of stars leaving the main sequence, because the time the stars spend in the giant branch in very small compared to that in the main sequence. Therefore, given that the colour evolution depends on the ratio of the number of stars in the main sequence to those in the giant branch and not on their absolute numbers, the colour evolution is mild and nearly independent of the initial mass function.

Therefore, to characterize the evolution of the elliptical galaxies in clusters, as described above, we need to observationally determine the following quantities:

1. The luminosity evolution of ellipticals in a timescale comparable to an important fraction of the age of universe.

2. The galaxy luminosity functions for clusters at moderate/high redshifts.

3. The intrinsic dispersions in the colour-magnitude relation for elliptical galaxies 
in moderate/high redshift clusters.

4. The dispersion in the mean galaxy colours for a set of different clusters at intermediate/high redshift.

5. The evolutionary colour vector between clusters at intermediate/high redshift and local clusters.

These observational numbers are highlighted in the text when they are first determined.

\subsubsection{Observational approach}

We decided to obtain high precision photometry for the red galaxies in a sample of $\sim 10$ clusters of galaxies at the highest possible redshifts which were accessible from the southern skies. This additional constraint was included in order to make follow up observations from the observing facilities available in Chile. These constraints defined the redshift of the sample to be $z \sim 0.4-0.5$. The final sample contained 8 clusters.

In order to be sensitive to small amounts of star formation that could affect the dispersion and evolution of the colour-magnitude relation, we require one of the photometric bands to be at restframe wavelength shorter than the $4000 \AA$ break. We included an infrared colour to study the colour evolution over a wide baseline in the wavelength allowing us to sample stellar populations of different effective temperature. Therefore, our data consist of deep $V, I$ and $K$-band imaging of the central regions in the fields of 8 clusters at $0.39<z<0.48$.

We require about $2 \%$ photometric uncertainties in the restframe $(U-V)$ colours of the galaxies down to 2 magnitudes below an unevolving $L^{*}$ galaxy at these redshifts. These photometric goals could be met in about 3 hours exposure time in the $V$-band and 1 hour exposure time in the $I$-band, using a $4 \mathrm{~m}$ class telescope.

To make the best use of the data we decided to included a novel approach to the photometry. The colours are obtained in a small fixed aperture and also from 
a 2-dimensional galaxy profile fitting procedure that takes into account the effects of seeing, and parallels that of DAOPHOT (Stetson 1987) for stars. This code has been developed by David Schade and modified by myself to be used within the IRAF environment. This code not only allows us to obtain accurate galaxy colours, but also provides morphological information on the galaxies by identifying different light profiles with galaxy morphologies. Gaining morphological information for galaxies at $z \sim 0.4-0.5$ from ground based observations under 1 arcsec seeing conditions is challenging.

Our observed $(V-I)$ and $(I-K)$ colours for the galaxies at $z \sim 0.45$ closely correspond to the restframe colours $(U-V)$ and $(V-H)$. These two colours are sensitive to different stellar populations in the galaxies. While $(U-V)$ is more sensitive to the main sequence stars, $(V-H)$ is dominated by the stars in the red giant branch. Assuming that the ellipticals in the clusters at $z \sim 0.45$ evolve into the ellipticals seen in local clusters today, it is possible to have two observations in the global evolutionary histories of these galaxies. We can, therefore, construct the evolutionary vector in the $(U-V) /(V-H)$ colours between the epoch at $z \sim 0.45$ and the present. As these two colours are dominated by different stellar populations, they provide a powerful test for spectral synthesis models of elliptical galaxies.

To detect the luminosity evolution, we take two different approaches using the two datasets available from this work. First, by using the 2-dimensional galaxy profile fitting code introduced above for galaxies in the fields of rich HST clusters, we can study the evolution of the size-magnitude relation for elliptical galaxies (Kormendy 1977). Second, by computing directly the galaxy luminosity function for the red galaxies in the sample of clusters at $z \sim 0.45$, we can compare the characteristic luminosity with local determinations.

\subsection{Thesis outline}

In this thesis I present the results from a comprehensive study of the elliptical galaxies in the fields of eight clusters of galaxies at $0.39<z<0.48$. The observations 
have been taken in the optical and infrared, using the CTIO $4 \mathrm{~m}$ telescope. This ground based dataset has been supplemented with 10 clusters of galaxies at $0.2<$ $z<1.2$, for which archival HST imaging was available. The description of the ground based observations, reduction, photometric techniques and images for the cluster fields is presented in Chapter 2. The analysis of the morphology and its application to the study of the surface brightness evolution of the ellipticals in the archival HST imaging dataset is presented in Chapter 3. The luminosity function for the red galaxies in the fields of the CTIO clusters is presented in Chapter 4, and it is compared to local determinations to verify the luminosity evolution detected using the surface brightness analysis. In Chapter 5, I discuss the dispersion (within and among clusters) in the colour-magnitude relation for the clusters at $z \sim 0.45$. This analysis sets constraints on the dispersion in the formation of these galaxies when compared to their mean light-weighted age. In Chapter 6, the restframe $(U-V) /(V-H)$ evolutionary vector is constructed from our observations and published photometry for the galaxies in Coma. This vector is used to test the predictions of several evolutionary spectral synthesis models. In Chapter $7, \mathrm{I}$ present a summary and discuss the observations in the context of our current knowledge of galaxy formation. In this work we assume $q_{0}=0.5$ and $H_{0}=50 \mathrm{~km} \mathrm{~s}^{-1} \mathrm{Mpc}^{-1}$, unless otherwise stated. 


\section{Chapter 2}

\section{Observations}

In this chapter I describe the steps involved in the creation of a photometric catalog for galaxies in the field of eight clusters of galaxies. I describe the cluster sample and its selection criteria, the reduction and calibration of optical and IR data, the determination of magnitudes and colours and finally I show the $I$-band images centered on the clusters in this study.

\subsection{Sample selection}

The observations for this thesis work have been designed to characterize the earlytype galaxy population in clusters at a significant look-back time. This population can then be compared with their local counterparts.

We chose to observe rich clusters of galaxies located in the southern hemisphere in order to build a database that could be used for follow up observations in the future using all the facilities available in Chile, including the VLT. Unfortunately, this constraint severely limits the number of moderate/high redshift clusters that could be included in this work. Most of the high redshift cluster surveys have been done (at least until the moment of the observations in this thesis) in the northern sky (e.g., Gunn et al. 1986). Therefore, building a sample of moderate/high redshift clusters imposed an extra challenge for this thesis.

The availability of clusters in the southern skies defined several of the criteria to 
create the sample. The clusters in this work were then compiled according to the criteria (in order of increasing importance) what they must:

1. Have a high surface density contrast of galaxies.

2. Have at least two galaxies of known redshift in the cluster.

3. Have an X-ray measurement to confirm there is a real association of galaxies and not only a line-of-sight superposition.

4. Have $0.40<z<0.50$. This redshift range was selected, as mentioned before, because:

- It is the highest redshift with a large sample $(N>10)$ of clusters (at least at the moment of the observations).

- The small redshift range minimizes the relative $k$-corrections between clusters, making the comparison easier.

- The $U V$-flux shifts into the high gain region of the CCD, and $V I K$ is well matched to $U V K$.

- The size of the clusters matches nicely the size of the CCD (1 Mpc at $z=0.45$ is $\sim 150$ arcsec, $1 / 5$ of the chip on a side).

We had initially selected a sample of 19 clusters that met the above requirements. From this pre-selected sample we actually observed 8 galaxy clusters which are the subject of the present study. This sample is presented in Table 2.1. All the clusters, except number 15, were selected from Couch et al. (1991). Cluster 15 was selected from Schindler et al. (1995).

The redshift, position and over-density contrast $(\sigma)$ were taken from Couch et al. (1991) for the clusters selected from that work. Their X-ray luminosities are from Bower et al. (1994). For cluster number 15 (c15), the position and redshift are taken from Schindler et al. (1995) and the X-ray luminosity from Schindler et al. (1997). 
Table 2.1: Cluster sample observed in this thesis work

\begin{tabular}{clcccccc}
\hline \hline $\mathrm{N}$ & Name & $z$ & $\mathrm{RA}(1950)$ & $\operatorname{Dec}(1950)$ & $\sigma$ & $\mathrm{N}_{z}$ & $\mathrm{~L}_{\mathrm{X}}$ \\
& & & & & & & $10^{44}$ \\
\hline 4 & J2175.15TR & 0.396 & $03: 32: 29.7$ & $-39: 03: 58$ & 5.1 & 12 & 0.648 \\
5 & J1556.15BL & 0.457 & $03: 37: 31.0$ & $-35: 38: 56$ & 5.6 & 3 & $(0.473)$ \\
6 & F1652.20CR & 0.411 & $04: 45: 52.4$ & $-20: 42: 50$ & $4+$ & 12 & 0.396 \\
8 & J2001.21C & 0.413 & $05: 12: 14.4$ & $-48: 21: 54$ & 4.7 & 3 & $(0.142)$ \\
11 & J1834.8BL & 0.445 & $10: 43: 18.6$ & $+00: 17: 31$ & 4.4 & 2 & 0.807 \\
12 & F1835.22CR & 0.471 & $10: 44: 28.4$ & $-00: 11: 17$ & 4.9 & 9 & 0.228 \\
15 & RX1347.5-11.45 & 0.451 & $13: 44: 50.6$ & $-11: 30: 12$ & & 2 & 73.0 \\
19 & F1637.23TL & 0.476 & $23: 56: 42.5$ & $-32: 34: 02$ & 5.2 & 10 & 0.496 \\
\hline
\end{tabular}

$\mathrm{N}$ is the reference number given in this thesis work.

$\sigma$ is the density contrast as defined in Couch et al. (1991).

$N_{z}$ is the number of redshifts in the cluster.

The X-ray luminosity, in the 0.1-2.4 keV band, given in units of $10^{44} \mathrm{erg} \mathrm{s}^{-1}$.

\subsection{Optical data}

For the completion of this program a total of 14 nights were awarded at the Blanco 4-m telescope at Cerro Tololo Inter-American Observatory (CTIO) between 1995 and 1996. Of these 14 nights, 8 corresponded to the optical part of the program. The summary of the observations is given in Table 2.2.

The optical data was obtained using the $2 \mathrm{k} \times 2 \mathrm{k}$ CCD detector Tektronix \#4 at the prime focus of the CTIO $4 \mathrm{~m}(\mathrm{f} / 2.85)$. This setup produces a $0.43 \mathrm{arcsec} / \mathrm{pix}$ plate scale, or equivalently a field of view of $14.7^{\prime} \times 14.7^{\prime}$. The CCD has good response throughout the whole optical region, as well an excellent cosmetic quality. We used the instrument with a gain setting of 2 , which produces an effective gain of $2.9 \mathrm{e}^{-} / \mathrm{ADU}$, and a readout noise of $4.2 \mathrm{e}^{-}$. The CCD was read in "quad mode" in order to take advantage of the four amplifiers and keep the readout time as low as $\sim 30 \mathrm{sec}$ for each image.

The images were obtained in $V$-band and $I$-band using the respective filters from the Harris and Kron-Cousins sets available at CTIO. The transmition curves for these two filters are shown in Figure 2.1. 
Table 2.2: Details of the optical observations for the clusters in this work.

\begin{tabular}{|c|c|c|c|c|c|c|c|c|c|}
\hline \multirow[b]{2}{*}{ cluster } & \multicolumn{3}{|c|}{ Nov95 } & \multicolumn{3}{|c|}{ Mar96 } & \multicolumn{2}{|c|}{ Dec96 } & \multirow[b]{2}{*}{ total } \\
\hline & $\mathrm{n} 1$ & $\mathrm{n} 2$ & n3 & n1 & $\mathrm{n} 2$ & n3 & n1 & $\mathrm{n} 2$ & \\
\hline \multirow[t]{2}{*}{$\mathrm{c} 4$} & 35 & $\overline{4}$ & 12 & - & - & - & 16 & - & 67 \\
\hline & 11 & 4 & 1 & - & - & - & - & 16 & 32 \\
\hline \multirow[t]{2}{*}{ c5 } & - & 4 & 29 & - & 3 & - & 16 & - & 52 \\
\hline & - & 12 & 1 & - & 3 & - & - & 16 & 32 \\
\hline \multirow[t]{2}{*}{ c6 } & - & - & - & - & - & - & - & 36 & 36 \\
\hline & - & - & - & - & - & - & 32 & - & 32 \\
\hline \multirow[t]{2}{*}{ c8 } & - & - & - & - & 8 & 24 & - & 4 & 36 \\
\hline & - & - & - & 16 & 16 & - & 4 & - & 36 \\
\hline \multirow[t]{2}{*}{ c11 } & - & - & - & 16 & 32 & - & - & - & 48 \\
\hline & - & - & - & 16 & 1 & 16 & - & - & 33 \\
\hline \multirow[t]{2}{*}{ c12 } & - & - & - & 16 & 15 & 8 & - & - & 39 \\
\hline & - & - & - & - & 8 & 24 & - & - & 32 \\
\hline \multirow[t]{2}{*}{ c15 } & - & - & - & 27 & 1 & 16 & - & - & 44 \\
\hline & - & - & - & - & 15 & 16 & - & - & 31 \\
\hline \multirow[t]{2}{*}{ c19 } & - & - & 40 & - & - & - & 16 & - & 56 \\
\hline & 15 & - & 1 & - & - & - & - & 16 & 32 \\
\hline
\end{tabular}

This table shows the number of images obtained for each cluster, in the $I$ and $V$-band, respectively. n1,n2, and n3, correspond to the first, second and third night during the observing runs in November 1995, March 1996, and December 1996. The last column gives the total number of images acquired during these three runs. The integration time for each image is 180 seconds.

\subsubsection{Observing strategy}

Obtaining high precision photometry for the galaxies in these clusters imposes a strong constraint on the observing strategy and the treatment of the systematics introduced by the observing procedure. We decided to aim for a few percent ( 23) photometric errors in red galaxies that are 2 magnitudes fainter than $M^{*}$ at the cluster redshift. This goal is achieved by exposure times of $\sim 8600 \mathrm{sec}$ in $V$-band and $\sim 5700 \mathrm{sec}$ in $I$-band, and a careful treatment of the response of the CCD, through flatfielding.

We used the shift-and-stare technique (Tyson 1986) to determine the response of the CCD, by creating superflats from the object images themselves. This technique allows to flatfield the sky to great accuracy, minimizing the uncertainties in the sky variations and thus minimizing the main source of errors in faint galaxy photometry. 


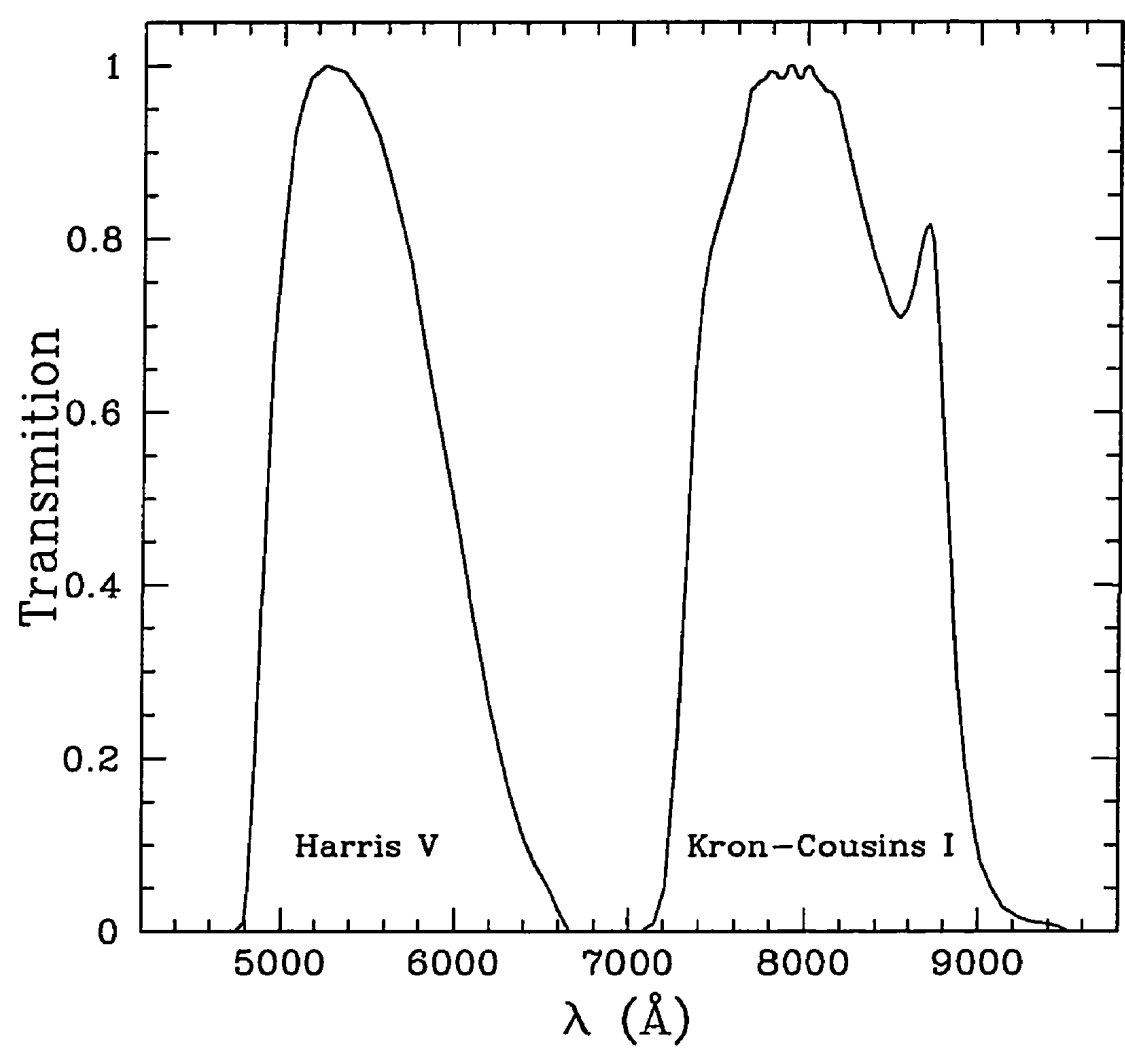

Figure 2.1: Transmition curves for the $V$ and $I$ filters used in the work. These filters correspond to the Harris and Kron-Cousins sets, respectively.

The superflats are created from the response to sky light, avoiding the mismatch in wavelength that a domeflat would introduce. The superflat technique works well as long as the spatial shifts are larger than the objects of interest and there are enough images to create the superflats. For this purpose we used blocks of 16 images, $180 \mathrm{sec}$ exposure time for each image, to observed each cluster in a given night. The images were spatially shifted in steps of 20 arcsec within each of these 16-image blocks. This procedure produces a well sampled region of $\sim 12^{\prime} \times 12^{\prime}$ for each cluster target. We normally obtained, two of these 16-image blocks in the $I$-band, and three in the $V$-band. Some of the clusters were observed in several runs using shorter blocks, and are used to compare the photometric calibrations. 


\subsubsection{Generation of superflats}

All the optical images were reduced in two stages using $\operatorname{IRAF}^{1}$ v2.10.4: 1) a simple standard reduction, and 2) the creation of and division by individual superflats. The standard reduction consists of the removal of the overscan and bias image, and the division by their corresponding domeflat. The second stage consists of the creation of individual superflats to be applied to the images. This later procedure becomes effectively a small but important correction.

To create the superflats for each of the cluster images we simply median combine the images in each of the 16-image blocks, taking the precaution of excluding the image for which we are creating the superflat. These superflats are then normalized and applied to the images. Bright objects in the images bias the median combined images, and we therefore mask these objects in order to minimize their impact on the superflat. Thus, in detail we implemented the following procedure to create the superflats:

1. Create a temporary cluster image by registering and combining all the available images for each cluster, in a given observing run. Registration was done in shifts of integer values and the image combination with an average including a 3- $\sigma$ clipping algorithm to eliminate cosmic rays.

2. Create an exposure-time map for this temporary cluster image to be used by the object detection algorithm. The center region in this combined cluster image is exposed the most, while the exposure time declines toward the edges of the image. This is produced because the overlap region between the different images corresponds to the center of the combined image.

3. Determine the sky and noise level in the well sampled region at the center of the image, and scale it to the different areas in the temporary cluster image. Then run an object detection algorithm, pixel by pixel, at a 3- $\sigma$ level above the

\footnotetext{
${ }^{1}$ Image Reduction and Analysis Facility (IRAF) is written and supported by the IRAF programming group at the National Optical Astronomy Observatories (NOAO) in Tucson, Arizona
} 
sky value. This procedure then creates another temporary image with pixels having values 1 or 0 , depending on whether the pixel in the cluster image had a value greater than the 3- $\sigma$ level above the sky or not. This image flags the positions were there is an object detected at the 3- $\sigma$ level.

4. We then create a third temporary image by running a moving average filter of size $10 \times 10$ pixels on the image that flags the position of the objects at the $3-\sigma$ level. This procedure is used to smooth the detection image.

5. Next we run a second object detection algorithm on the smoothed image, flagging the pixels above a threshold level that varies between 0.08 to 0.10 (recall that the detection image created above has pixel values that range between 0 and 1). The image created here is then used as a mask, where all the stars and galaxies, including their faint wings, have been detected and flagged. This procedure flags typically $10-15 \%$ of the pixels in each of the images.

6. The mask image for each cluster is then used to create individual mask images associated with each of the cluster images. These are just made by deregistering the main mask image appropriately.

7. The superflat image for each of the images, is then created by median combining the images in a block of 16 images, including their corresponding masks and excluding the image for which the superflat is being created. It is necessary to exclude the image from its own superflat, otherwise a fraction $1 / N$ pixels (where $N$ is the number of images being combined) will come from every image. And therefore, $1 / N$ pixels will be divided by themselves, introducing a bias on those pixels. These superflats images are normalized to unity.

8. We finally divide all the images by their corresponding superflats, to obtain the images free of systematics.

Once all the images in both bands have gone through this process they are ready for the next step in their reduction, the correction for image distortions. 


\subsubsection{Image distortions}

It was necessary to apply geometric corrections to the single images due to two effects: (a) a simple translation would produce offsets of 1-2 pixels at the edges of the field, which would introduce photometric biases when the seeing is only 2.5 pixels; (b) images from the November 1995 run have a slightly different scale $(0.6 \%)$ than the images taken in March 1995 and December 1996.

We used geomap (available in immatch in IRAF) and 120 stars in the common area of the images for each cluster to determine the distortions in each of the images. The distortions were characterized by a second order polynomial where the coefficients could be identified with a linear shift, a rotation, and a change of scale. The rms for these fits was typically 0.2 pixels. We used one of the $I$-band images for each cluster as a reference for all the rest of the images, including the $V$-band images.

After the geometric corrections were computed, we used geotran to correct the images to a common spatial system. We constrained the algorithm to conserve flux in order to keep it the same as in the original image.

\subsubsection{Photometric calibration}

Up to this point all the images have been treated to remove the sensitivity variations from pixel to pixel and also the geometric effects introduced by the optical system. The next step consists of calibrating these images in order to be able to combine them and produce the final images for each cluster field.

To determine the calibration for our images, we observed standard stars from Landolt (1992). We selected faint stars in order to avoid saturation of the CCD, and we also required red stars to be in the fields so that the colour calibration could be determined reliablely for the red galaxies in our observing program. The standard fields we observed contain typically between 5 and 30 standard stars in the $15^{\prime} \times 15^{\prime}$ covered by the CCD. As the stars in these fields have a wide range in brightness, we decided to observe them (for both bands) using three different exposure times, normally 2,4 and 8 seconds. 


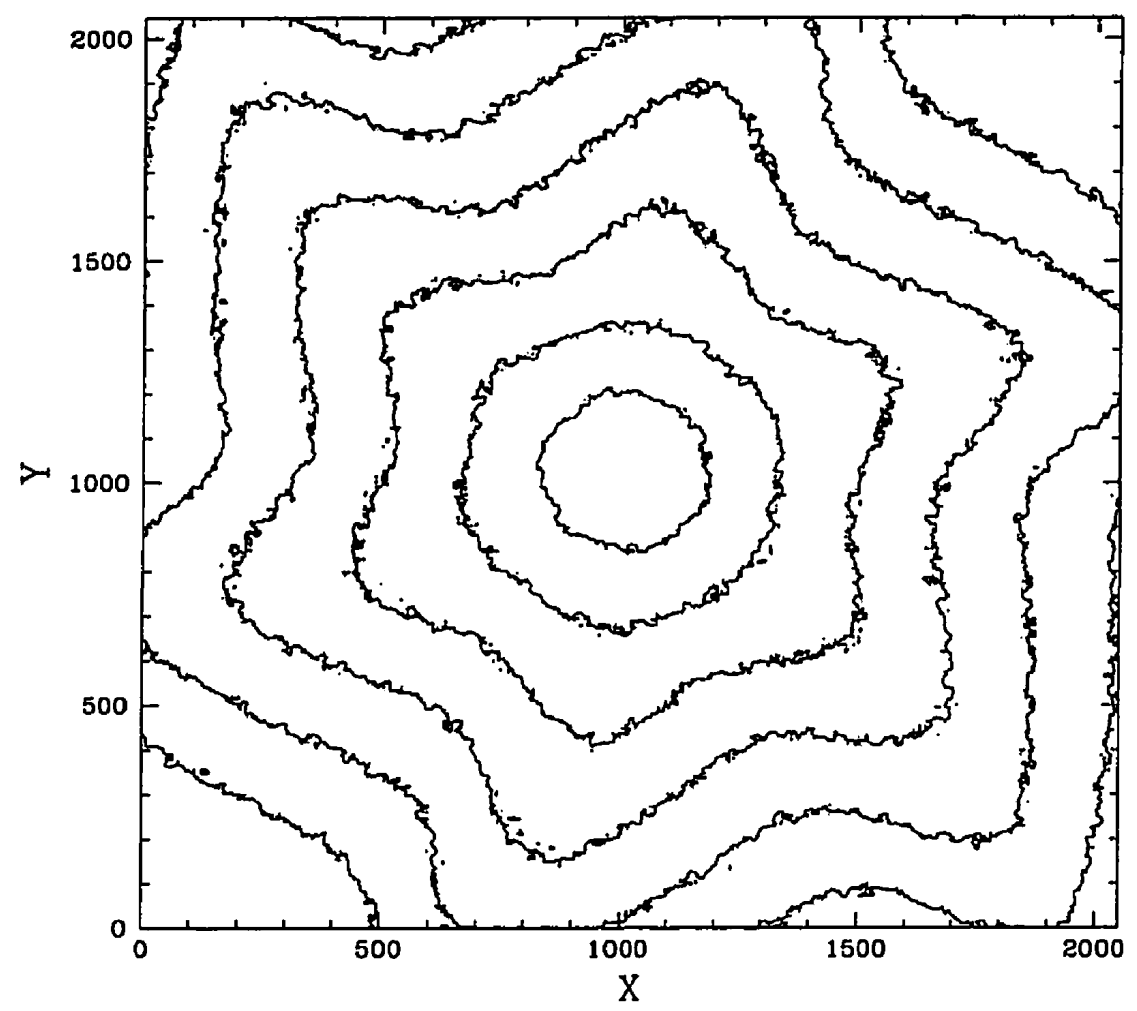

Figure 2.2: Exposure delay image for the optical images in this work. The field of view is unevenly exposed because the shutter opens from the center and takes a finite time to close. The contours show a time delay of 10, 20,30,40,50 and $60 \mathrm{~ms}$ at the center, i.e. the exposure time in the center is $\sim 60 \mathrm{~ms}$ longer than at the edges. This image also shows the shape the shutter blades take when they are activated.

We observed the standard stars three to four times during each of the observing nights, sampling a wide range in colour, brightness and airmass. The standard stars were selected by trying to match the colour of the ellipticals at $z \sim 0.45$, i.e. $(V-I) \sim$ 2. These observations produced typically about 300 reference star observations per night to determine the extinction and transformation coefficients. Some of the stars were observed several times.

As the exposure time for the observations of the standard stars was only a few seconds, and the shutter had to move over a large aperture, these observations could be subject to an uneven illumination that affects the photometry of the standard 
stars. This effect arises because the center of the chip is exposed to sky longer than the edges. To estimate the amount of this effect we obtained a $n$ second-exposure domeflat $(n=20)$ and we compare it with an image that has been exposed $n$ times during 1 second (without reading the CCD). Using the ratio of these two images, it is possible to produce an exposure map $\left(\delta_{i j}=n\left(R_{i j}-1\right) /\left(n-R_{i j}\right)\right)$ showing the shutter delay (i.e. the effective amount of time the shutter remains open above the requested time) at any point in the image. We found that the center of the CCD remains open for $60 \mathrm{~ms}$ longer than the edges, as can be seen in Figure 2.2. This effect could produce a magnitude difference of up to $3 \%$ (for a 2 seconds exposure time image) if the stars are near the center of the field.

To correct for this differential exposure time we smoothed the exposure map image and created an exposure correction image for each of the standard star images. The pixel $(i, j)$ in the corrected image takes the value $c_{i j}=t /\left(t+d_{i j}\right)$, where $t$ is the exposure time (in seconds) of the image being corrected and $d_{i j}$ is the pixel $(i, j)$ in the exposure map image.

Once the images for the standard stars have been corrected for the shutter delay image, we are in a position to obtain photometry for these stars. In order to avoid saturation for these relatively bright stars we observe them with the telescope slightly out of focus. This implies that we need to determine the magnitude in a larger aperture than measured in Landolt (1992). We determined a 20" aperture magnitude for the standard stars by computing a $12^{\prime \prime}$ aperture magnitude corrected using the light profile of a few bright isolated reference stars. We also measured the sky value in a 30-50" ring centered at the position of each star.

With the standard observations we determine the response of our system and we characterize it by using Equations 2.1 and 2.2, where $v_{1}$ and $i_{1}$ are the photometric zero points, $v_{2}$ and $i_{2}$ are the extinction coefficients, and $v_{3}$ and $i_{3}$ are the colour terms. To determine the extinction coefficients we used the cluster observations when a wide range in extinction is present, otherwise we use the standard stars observed at different airmasses. We solve the equations using photcal available in IRAF. 


$$
\begin{aligned}
& m_{V}=V+v_{1}+v_{2} X_{V}+v_{3}(V-I) \\
& m_{I}=I+i_{1}+i_{2} X_{V}+i_{3}(V-I)
\end{aligned}
$$

Solving these equations independently for each night of the three observing runs allows us to assess the photometric quality of the nights. We found that the runs of March and December 1996 were of excellent photometric quality, in contrast to our first run (November 1995) that was of variable quality. We therefore decided to calibrate the images taken in the November 1995 run using the observations of December 1996 as a reference -all the clusters of the November run were also observed in the December run, see Table 2.2-. We also had observations for two clusters in common between the March and December runs. When comparing the photometry for bright non-saturated stars in the fields of these two clusters for the two runs, we found that the difference in the photometric zero points amount to $0.2 \%$ and $-3.8 \%$ in the $V$-band and $I$-band, respectively. This effect was traced to the fact that the observations of the standard stars were done with the telescope in an out of focus position, with a bigger effect in the $I$-band. We decided to correct the $I$-band images from the March run in order to take them to the same level as the December run (already used as a reference to calibrate the November run). This procedure ensures that all the photometry is self consistent.

The quality of the photometric calibration is shown in Figures 2.3 and 2.4, where the observations for the standard stars during the photometric nights of the March and December 1996 runs are plotted. These graphs show the difference in the observed $V$ magnitude for the standard stars with respect to the standard magnitude from Landolt (1992). The figures on the right show the range in the colour where the calibrations were computed (ellipticals at $z \sim 0.45$ should have $V-I \sim 2$ ), and also the measured $(V-I)$ for the stars when compared to their standard value.

The photometric zero points, extinction and colour terms determined for the December 1996 run are: $v_{1}=4.345 \pm 0.008, i_{1}=5.291 \pm 0.013, v_{2}(\mathrm{n} 1)=0.130 \pm 0.003$, 

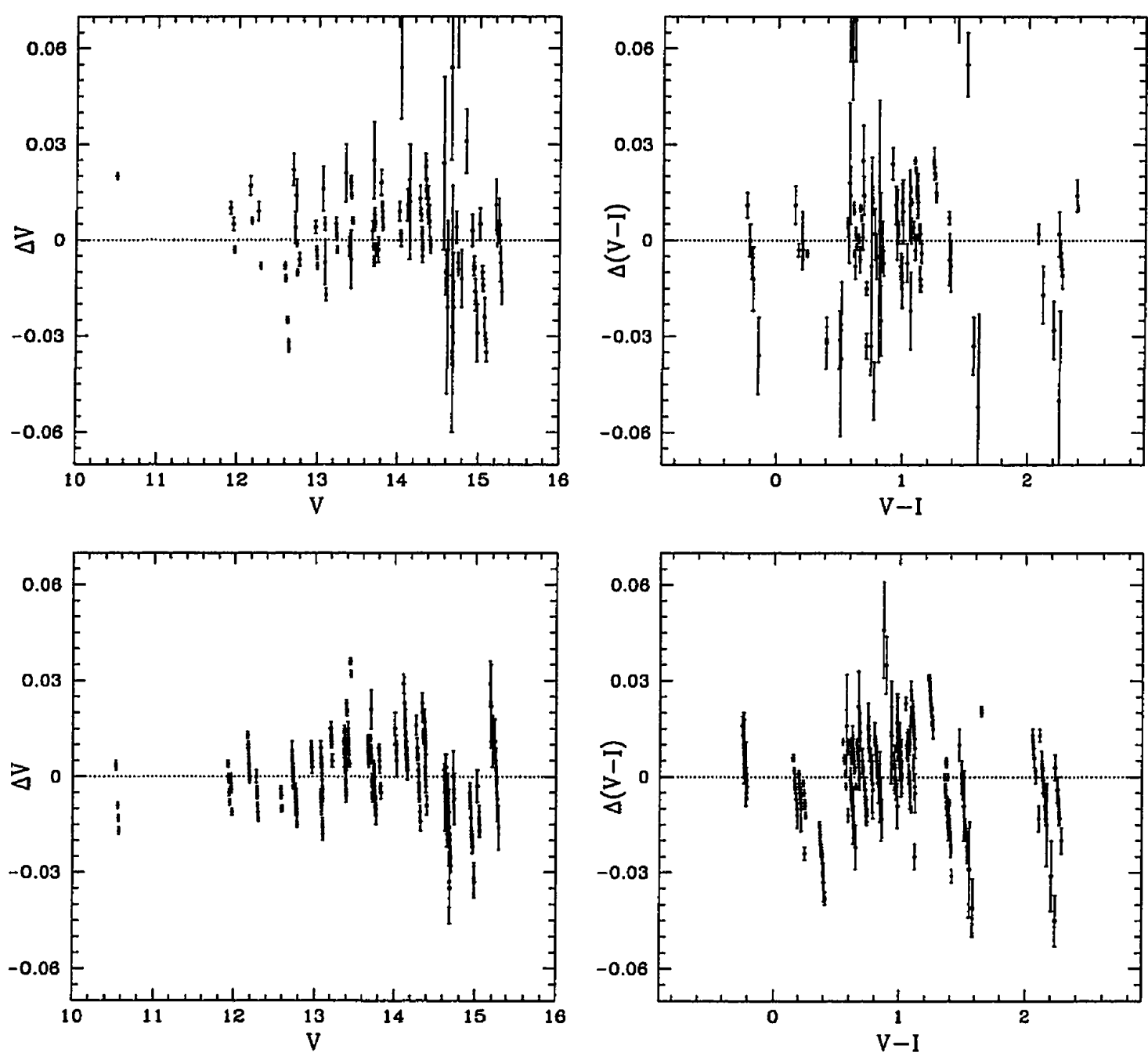

Figure 2.3: These figures show the quality of the optical calibration for the second (upper panel) and third nights (lower panel) during the March 1996 run. The first night of this run was not photometric. The left panels show the difference between the observed $V$ magnitudes in the images and the values from the catalog. The graphs at the right show the calibration in the $(V-I)$ colour for the same stars. There are $\sim 600$ observations of standard stars between these two nights, all these are shown here. These graphs also show there are no systematic errors, and the range where the calibrations were determined. 

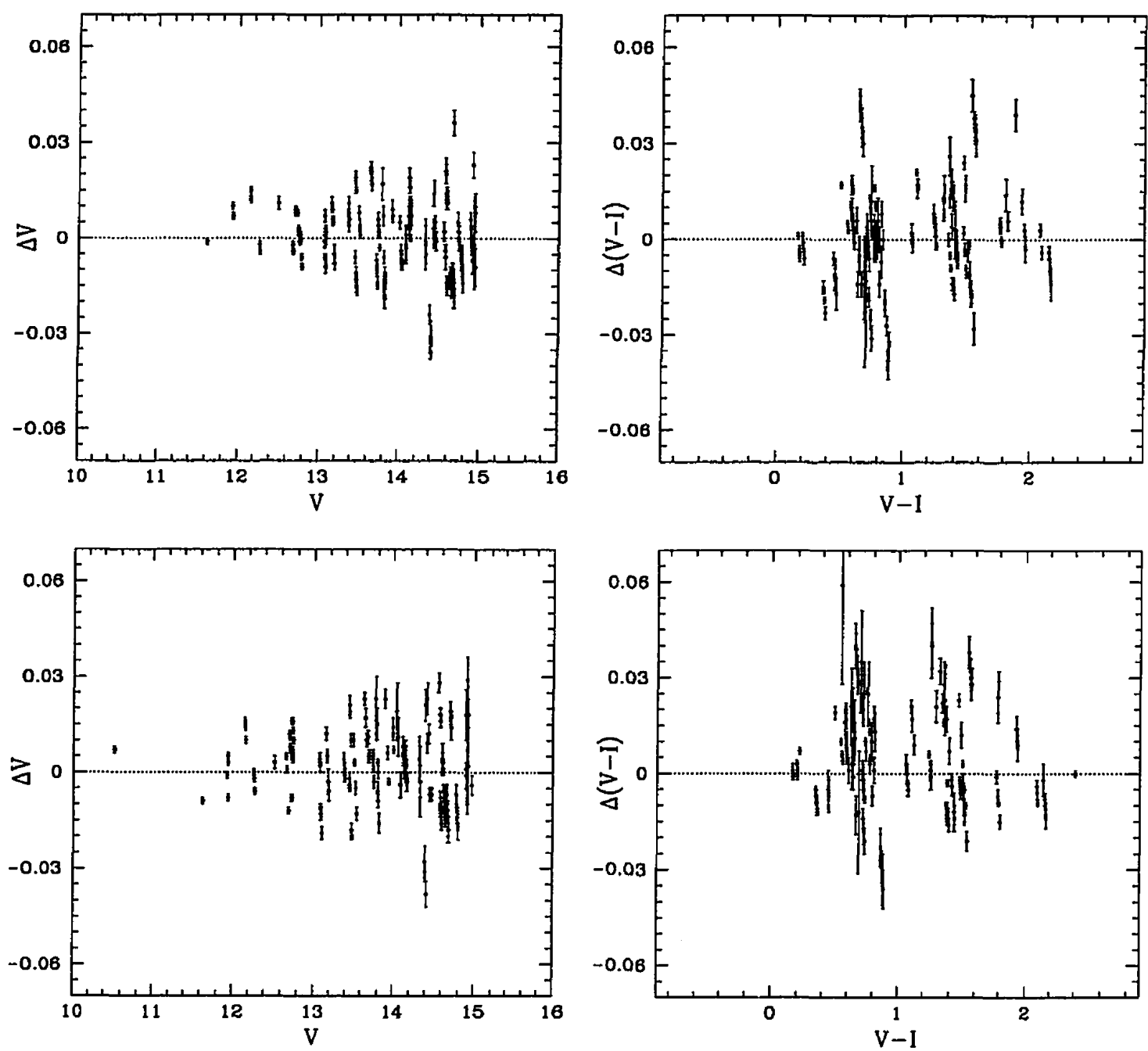

Figure 2.4: These figures show the quality of the optical calibration for the 1st (upper panel) and second nights (lower panel) during the December 1996 run. The left panels show the difference between the observed $V$ magnitudes in the images and the values from the catalog. The graphs at the right show the calibration in the $(V-I)$ colour for the same stars. There are $\sim 560$ observations of standard stars between these two nights. These graphs also show there are no systematic errors, and the range where the calibrations were determined. 
$v_{2}(\mathrm{n} 2)=0.131 \pm 0.003, i_{2}(\mathrm{n} 1)=0.044 \pm 0.003, i_{2}(\mathrm{n} 2)=0.049 \pm 0.004, v_{3}=$ $-0.020 \pm 0.002$, and $i_{3}=-0.012 \pm 0.002$.

\subsubsection{Combination}

After the images have been properly flatfielded, geometrically corrected, and photometrically calibrated they are ready for their combination into a single $V$-band and $I$-band image for each of the clusters.

We combined the images using an average of the images, including a sigma clipping algorithm for rejecting pixels with values deviating more than $3 \sigma$ from the median in the stack of images. We also weighted by the inverse of the rms in the sky of the images to increase the signal-to-noise ratio of the combined image.

\subsection{Infrared data}

The optical data in this program was supplemented with infrared imaging centered on the cluster fields. These data were taken in $K \mathrm{~s}$-band (transmition shown in Figure 2.5) using the CTIO $4 \mathrm{~m}$ and the CTIO InfraRed Imager (CIRIM). This instrument uses a $256 \times 256$ pixels $\mathrm{HgCdTe}$ NICMOS 3 detector, read using a double correlated sampling technique. The gain is $9 \mathrm{e}^{-} / \mathrm{ADU}$ and its readout noise $37 \mathrm{e}^{-}$. We used the instrument with a scale of $0.4 \mathrm{arcsec} / \mathrm{pix}(\mathrm{f} / 7.5)$, obtaining $102^{\prime \prime} \times 102^{\prime \prime} \mathrm{FOV}-\sim 700$ kpc at $z=0.45$. The data were taken during two runs, in March and November 1996.

The detector has a non-linear response that is characterized using Equation 2.3, with the values for the coefficients being: $c_{1}=0.99893, c_{2}=8.7807 \times 10^{-7}$ and $c_{3}=2.168 \times 10^{-11}$. The data can therefore be linearized by applying Equation 2.3 using the task irlincor in the CTIO package available in IRAF. This is the first correction to apply to the images, after the subtraction of the darks.

$$
\frac{I_{\text {corr }}}{I_{\text {obs }}}=c_{1}+c_{2} I_{o b s}+c_{3} I_{o b s}^{2}
$$

As the size of the detector was not large enough to cover the entire region of interest 


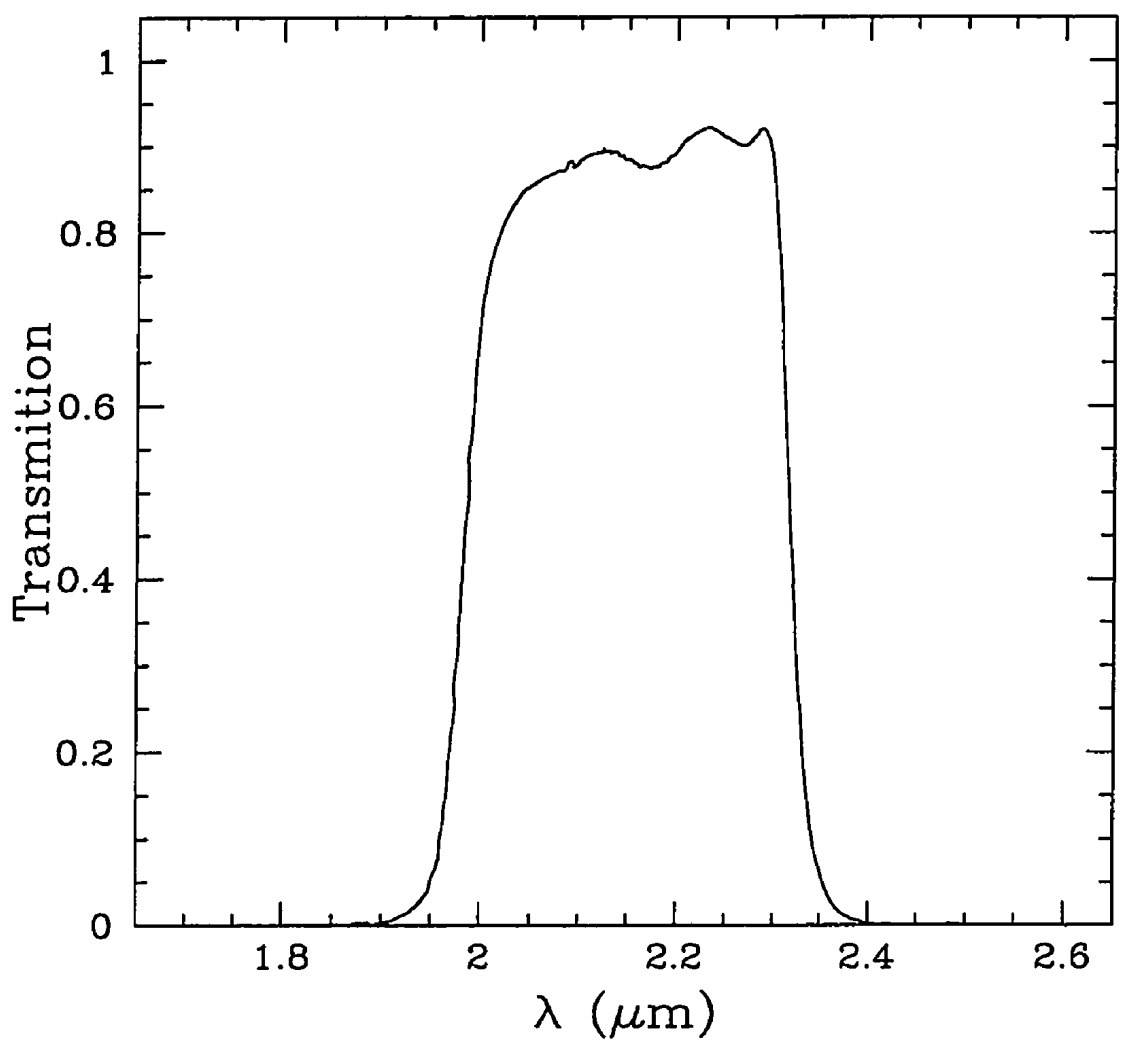

Figure 2.5: Transmition curve for the $K$-band filter used in the work. This filter corresponds to the $K$ s filter available at CTIO.

around the clusters, it was necessary to observed the targets using two superimposed mosaic patterns. To optimize the sampling for each cluster we observed 3-5 pointings each, covering the central 9 to 15 square arcmin of the clusters, with each pointing consisting of a smaller mosaic of $3 \times 3$ images with internal offsets of 10 arcsec. Each of these small mosaic patterns was repeated 6 times. The exposure time for each of the individual images was 60 seconds - 6 coadds of 10 seconds each-adding up to $\sim 1$ hour total exposure time for each of the pointings.

\subsubsection{Reductions}

The reduction of the IR data, as in the case of the optical data in this work, uses 
the data itself to remove their systematics ${ }^{2}$. Here I describe the steps involved in the reduction of this particular set of images.

1. Apply the linearity correction described above.

2. Create a sky image for all the images by median combining the 8 closest images in the sequence. The images are scaled to a common sky level.

3. Scale the sky image to the sky level of the target image and subtract the sky image from the target image.

4. Register all the images and combine them into each of the cluster mosaics. There was usually one or two objects to be used as reference to compute their spatial offsets.

5. Create an exposure map image in a similar way to the optical data. This allows us to scale the dispersion in the sky to all the regions in the combined image.

6. Compute the rms in the sky of the combined image and create a mask in the same way as for the optical data.

7. Create an individual mask for all the images based on the combined mask image.

8. Repeat the creation and subtraction of the sky image (steps 2 and 3), but now including the mask images in the combination of the data.

9. Apply the dome flat correction to all the images. Tests performed to the standard stars suggest that applying the domeflat image improves the photometric accuracy of the photometry by $2 \%$.

10. Apply the photometric correction computed from the standard stars (explained below).

11. Combine the sky subtracted and calibrated images to produce the final $K$-band image for each cluster.

\footnotetext{
${ }^{2}$ A good discussion about the treatment of IR data can be found in Joyce (1992)
} 
Table 2.3: Summary of the transformation coefficients for the IR data

\begin{tabular}{ccccccc}
\hline \hline & \multicolumn{3}{c}{ Mar96 } & \multicolumn{3}{c}{ Nov96 } \\
& n1 & n2 & n3 & n1 & n2 & n3 \\
\hline$k_{1}$ & 7.934 & 7.947 & 7.916 & 7.909 & 7.964 & 7.972 \\
$\sigma_{k_{1}}$ & 0.028 & 0.006 & 0.005 & 0.048 & 0.028 & 0.052 \\
$k_{2}$ & 0.129 & 0.094 & 0.124 & 0.092 & 0.071 & 0.071 \\
$\sigma_{k_{2}}$ & 0.020 & 0.000 & 0.005 & 0.040 & 0.021 & 0.040 \\
\hline
\end{tabular}

Zero points and extinction for the infrared observations during the different nights (n1, n2, n3) in the March 1996 and November 1996 runs. The colour term was found to be zero (within the errors), and it was set to zero for computing the zero point and extinction.

\subsubsection{Calibrations}

After correcting for the systematics in the $K$-band images we need to calibrate them to the standard system. For the observations in this work we have used the standard stars from the UKIRT compilation (Casali and Hawarden 1992).

The observations of the standard stars were obtained in three or four blocks during each of the observing nights. All the standard stars were placed in different positions in the detector to minimize the random errors and to estimate the effects of the flatfielding procedure. The magnitudes for these stars were determined in an aperture of $10^{\prime \prime}$ using the task phot in the package apphot available in IRAF.

$$
m_{K_{s}}=K+k_{1}+k_{2} X_{K_{s}}+k_{3}(H-K)
$$

To transform the instrumental magnitudes to standard magnitudes we used equation 2.4, were $m_{K_{s}}$ is the determined instrumental magnitude, $k_{1}$ is the zero point, $k_{2}$ and $k_{3}$ are the extinction and colour term, respectively. We determined the coefficients in this equation by using about 30-50 observations for the standards (per night), and the package photcal in IRAF.

The transformation coefficients can be determined independently for each of the observing nights, as it is shown in Table 2.3. These determinations can be improved by assuming the detector remained stable through the run and therefore we can take an average for the zero point $\left(k_{1}\right)$, as the fitted values suggest. With the average zero 
Table 2.4: Transformation coefficients used in the IR corrections.

\begin{tabular}{lcccc}
\hline \hline & $\begin{array}{c}\left\langle k_{1}\right\rangle \\
\text { error }\end{array}$ & $\begin{array}{c}k_{2} \text { (night 1) } \\
\text { error }\end{array}$ & $\begin{array}{c}k_{2} \text { (night 2) } \\
\text { error }\end{array}$ & $\begin{array}{c}k_{2} \text { (night 3) } \\
\text { error }\end{array}$ \\
\hline Mar96 & & & & \\
& 7.932 & 0.131 & 0.104 & 0.114 \\
& 0.013 & 0.004 & 0.004 & 0.004 \\
& & & & \\
\hline \hline Nov96 & & & & \\
& 7.954 & 0.054 & 0.080 & 0.085 \\
& 0.025 & 0.005 & 0.002 & 0.002 \\
\hline
\end{tabular}

Different night extinctions $\left(k_{2}\right)$ obtained by using an average zero point $\left(k_{1}\right)$ during each of the observing runs (March 1996 and November 1996).

point we recompute the extinctions that can effectively vary from one night to the next. These final values, used in the transformations, are presented in Table 2.4. The colour term was zero within the errors, so we therefore decided to set it to zero for the rest of the computations.

Once the extinction and the transformation coefficients have been determined, we correct all the cluster images to take them to zero airmass and to the standard system.

To test the accuracy of the calibration procedure we compare the photometry for one of the clusters that was observed in both observing runs. Taking the objects with $K<18$ (41 objects) in common in both observations we found an offset in the photometric calibration of $\Delta K=0.00 \pm 0.07$ between the two runs. This value makes us confident that the reduction and calibration procedures are accurate enough for the purposes of this program.

\subsection{Photometric catalogs}

Obtaining high precision photometry for the cluster galaxies in this cluster sample not only imposes a strong constraint in the treatment of the images, but also requires sophisticated software to extract the information we are looking for. For this purpose 
we decided to perform the photometry in two stages: a) using sextractor (Bertin and Arnouts 1996) in a first pass to detect the objects, determine their positions and measure their "total" magnitude, and b) in a second pass use apphot (available in IRAF) to determine the colours in a fixed metric aperture.

Sextractor detects objects in the image which has been previously sky subtracted and convolved with a kernel, in this case with a $3 \times 3$ pixel pyramid. Sextractor detects an object when a predefined number of contiguous pixels are above a certain threshold. For this work we require 5 connected pixels above $1.5 \sigma$ (1.5 of the standard deviation in the sky). Once the objects have been detected, their total magnitudes are estimated using an elliptical aperture. The size of the aperture is computed as 2.5 times the Kron radius (i.e. $2.5 \times r_{1}=2.5 \times \sum r I(r) / \sum I(r)$ ), and within this aperture there should be more than $90 \%$ of the object's flux - for stars and galaxies convolved with a gaussian seeing, nearly independent of the object magnitude- - If there is evidence for bias by a brighter companion, then the magnitude computed using an elliptical aperture is replaced by a seeing corrected isophotal magnitude.

Another important feature of sextractor is that it estimates the probability that the detected object is a star or a galaxy, according to the light profile. Sextractor computes the stellarity index which is proportional to this probability, with stars having a value near one and galaxies having a value near zero. This stellarity index is computed using a neural network that has been trained using the light profiles of more than $10^{6}$ stars and galaxies, simulated under different conditions of seeing, pixel-scale and detection limits. The only control parameter for the algorithm is the seeing FWHM which defines the intrinsic fuzziness of the objects in the image. As an example we show the stellarity index as a function of the observed magnitude for one of the cluster fields in Figure 2.6. As expected, at fainter magnitudes the confusion between a star and a galaxy increases. Using the colour-colour plane (see Figure 2.7) we found a clear segregation between stars and galaxies that agrees with that obtained from the stellarity index. We defined a galaxy as an object with the stellarity index lower than 0.95 , as shown by the broken line in Figure 2.6. At $I>22$ some stars are included in the galaxy sample, but most faint $(I>22)$ objects are 


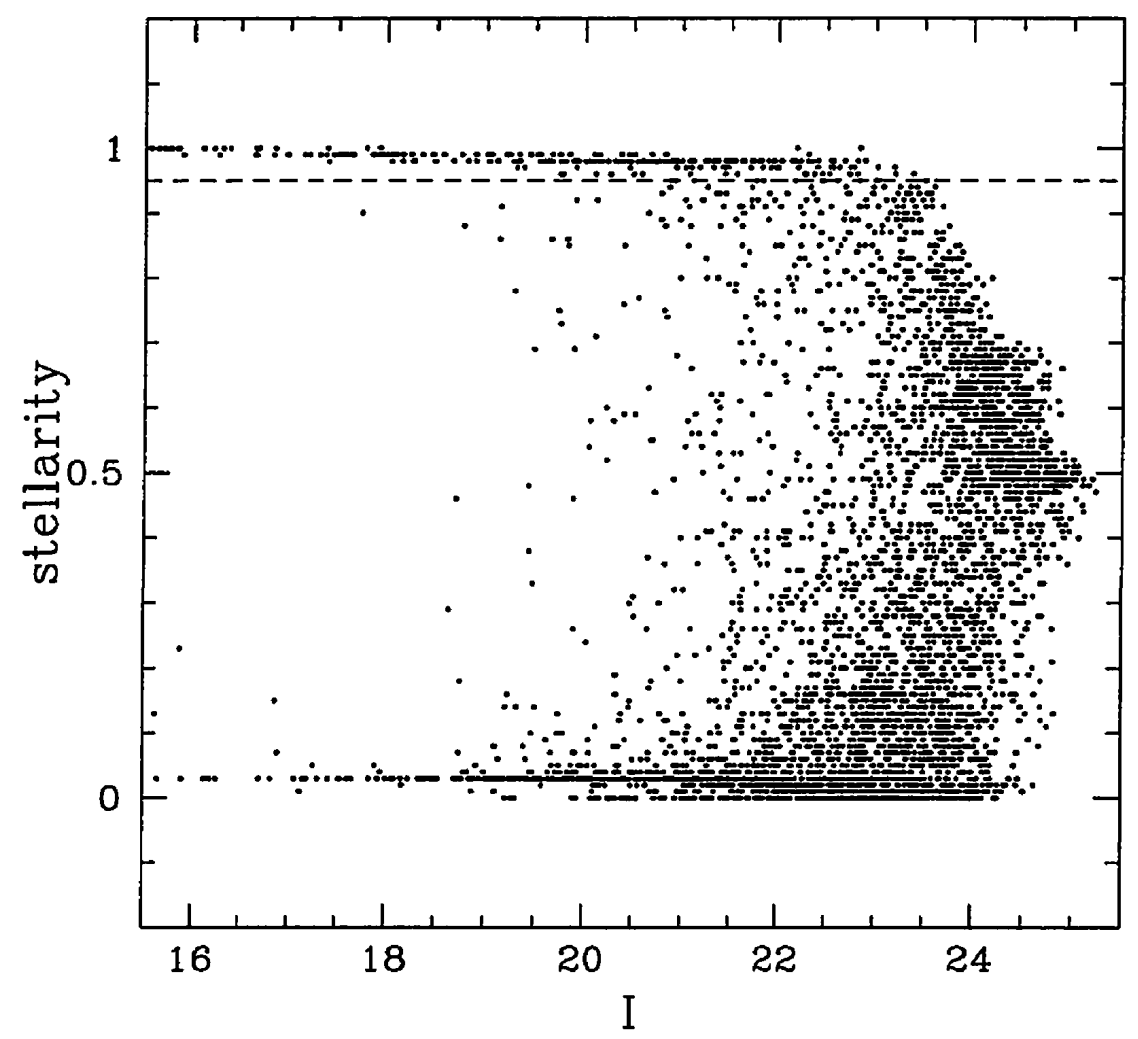

Figure 2.6: Stellarity index measured by sextractor in one of the cluster fields. The broken line at the index 0.95 divides the stars and the galaxies in this work. This boundary was determined from the segregation of stars and galaxies using the colourcolour diagram for the cluster fields

galaxies.

Once the positions and the magnitudes have been determined by sextractor then we use apphot to obtain the colours in a fixed aperture. We use an aperture of $3^{\prime \prime}$ centered in the position provided by sextractor to obtain the flux for each galaxy, and then compute the magnitude as the flux at the galaxy position minus a local value of the sky (which is determined in an annulus centered on the galaxy position). We decided not to recompute the center of the object in the other images because some of the objects could be considerably fainter in the $V$-band, as well as in the $K$-band, providing a poor determination of the position and thus affecting the galaxy colours.

Photometry in a small aperture to determine the colours of faint galaxies could 


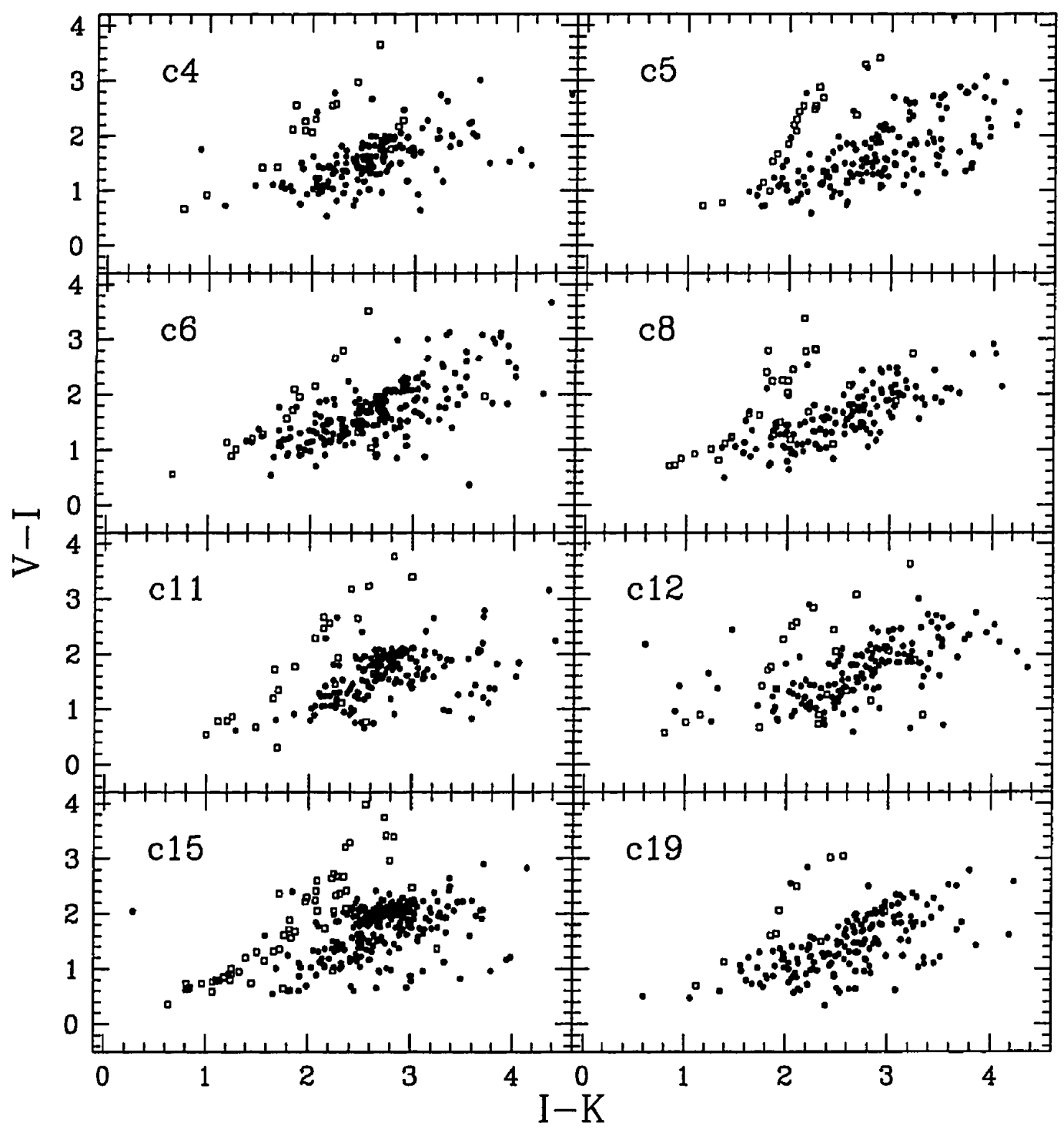

Figure 2.7: Colour-colour diagram for the objects in the cluster fields, down to $I=$ 23. These fields only include the area covered by the infrared observations, which correspond to the central regions of the clusters. Objects selected as stars, according to their light profile, are shown as open squares, and galaxies are shown as filled circles. Stars and galaxies are well separated by sextractor. 
be susceptible to a bias introduced by a mismatch between the point spread functions (PSFs) of the images involved. To eliminate (or reduce) this potential bias, we convolve the images with a gaussian kernel before determining the photometry. The convolution is made in the sense of bringing the best seeing images to the worst seeing image among the three bands, i.e. $V, I$ or $K$-band. The seeing for all the images (in all bands) ranges from $0.9^{\prime \prime}$ to $1.4^{\prime \prime}$. The maximum correction (compared to the non-seeing-corrected photometry) introduced by matching the seeing is 0.05 magnitudes in $(V-I)$ for the cluster $c 6$.

The colour determination not only imposes a strong constraint in the calibration procedure, but also in the correct assessment of the random uncertainties affecting the data. We are particularly interested in obtaining reliable errors in the $(V-I)$ colour because this will be used as a measure of the intrinsic dispersion in the galaxies in and among the clusters. These errors are determined empirically by determining the photometry for all the cataloged objects in two images created from half of the total number of images for each cluster (this is done for the $I$-band images and the $V$-band images). The error in the fixed aperture magnitude for a single galaxy is then half of the difference of the determined magnitudes, and the error in the colour is simply the errors in both bands added in quadrature. On the other hand, the errors in the $I$ magnitude determine by sextractor and the fixed aperture magnitude in the $K$-band were estimated statistically from the noise in the sky and of the flux in the object.

\subsection{Simulations}

To test the reliability of sextractor in finding the objects and the accuracy of the computed "total" magnitudes we performed some simulations by adding objects with known brightness to the cluster images.

We added to the cluster images typically 225 objects of a given light profile and brightness for each of the simulations, down to $I=26.25$ in steps of $\Delta I=0.5$ mag. These objects were created by using an exponential function for the disks and an $\mathrm{r}^{1 / 4}$ 


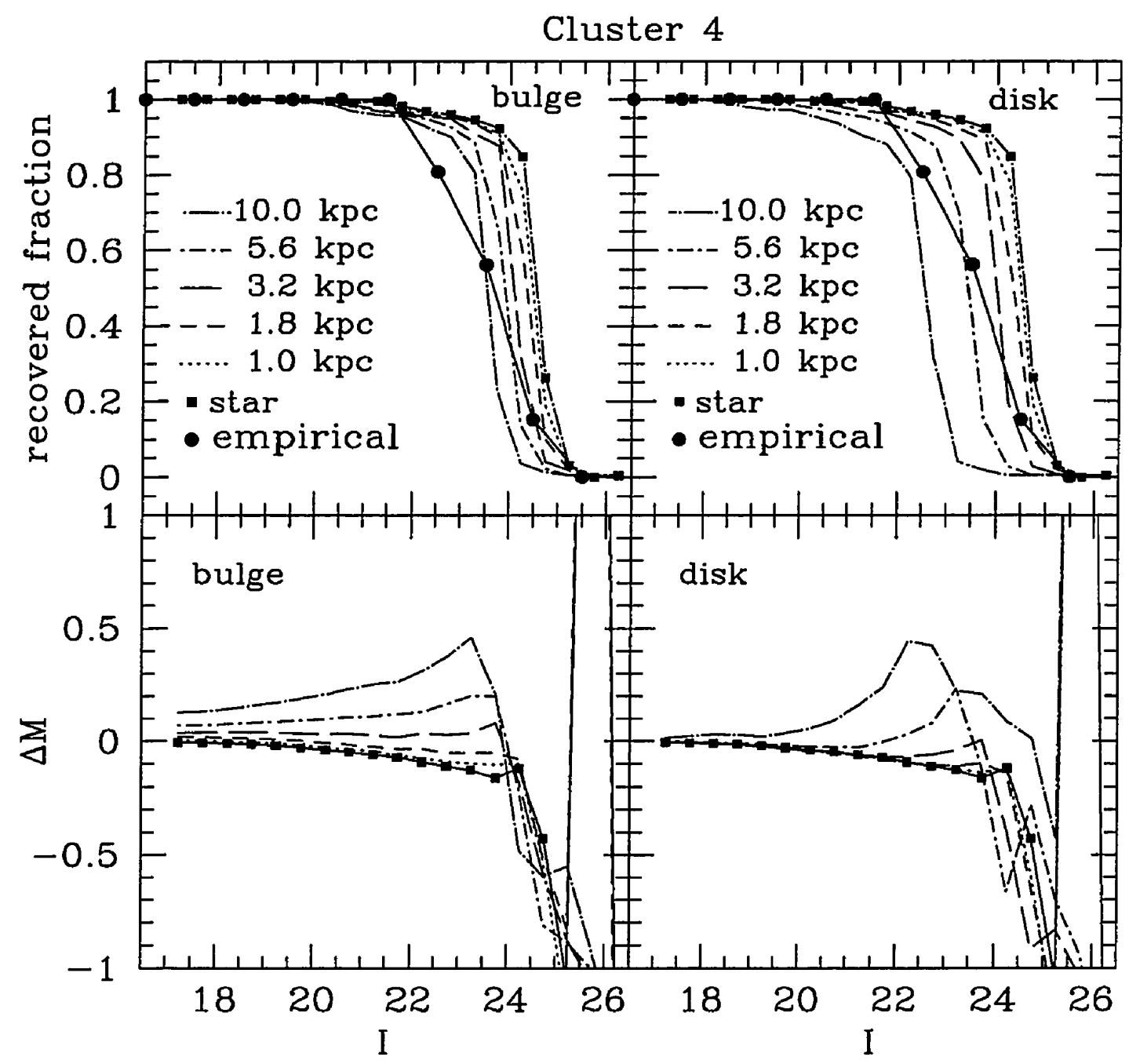

Figure 2.8: Simulations to obtain the detection rate and estimate the photometric accuracy of sextractor in the field of Cluster 4 . The different lines indicate the results for galaxies having different sizes (effective radius for bulges and exponential function for disks) at the redshift of the clusters. The solid line denotes the empirical recovered fraction determined in Chapter 4. 


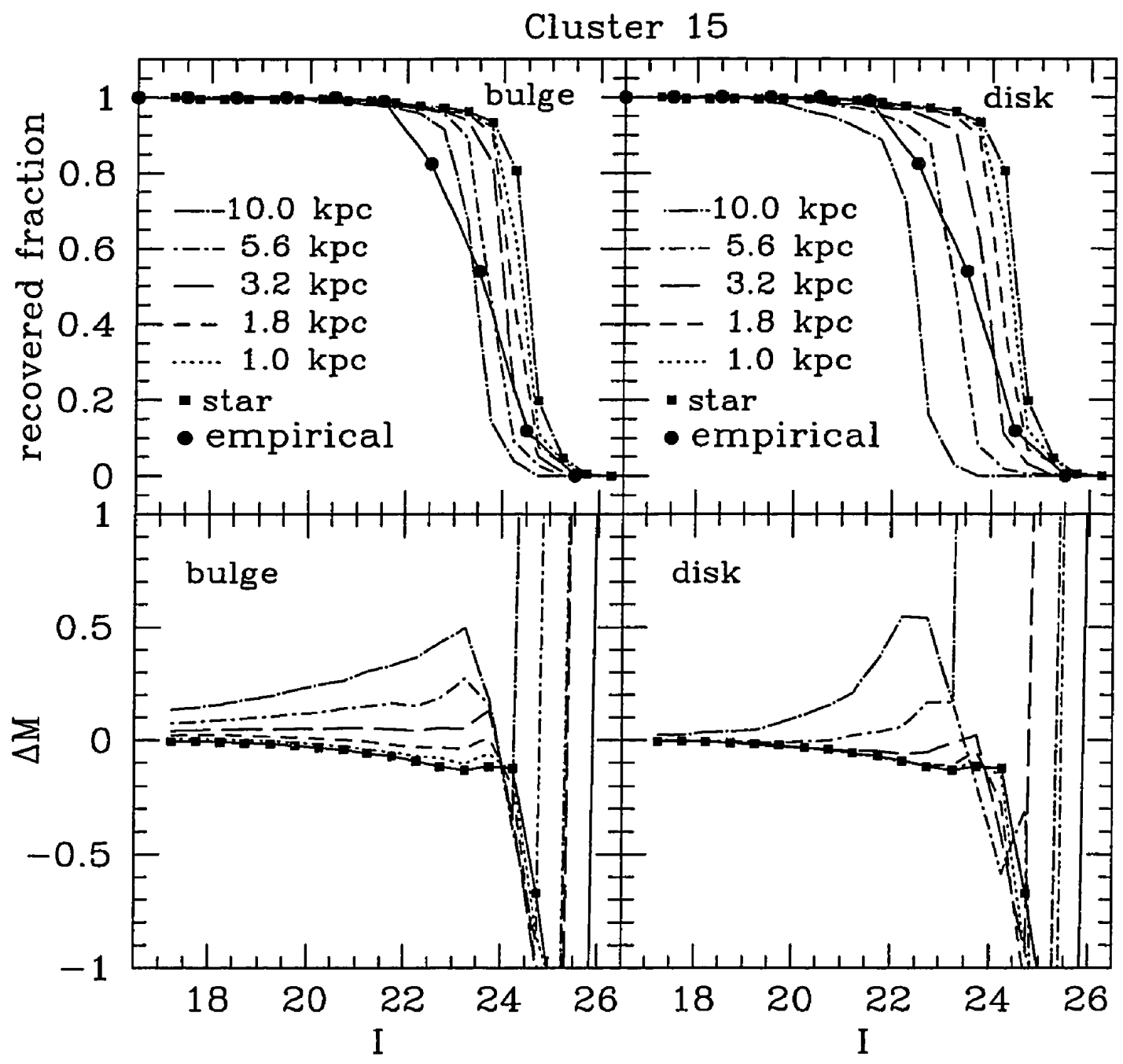

Figure 2.9: Simulations to obtain the detection rate and estimate the photometric accuracy of sextractor in the field of Cluster 15 . The different lines indicate the results for galaxies having different sizes (effective radius for bulges and exponential function for disks) at the redshift of the clusters. The solid line denotes the empirical recovered fraction determined in Chapter 4. 
law for the bulges (including different scale lengths), and then they were convolved with the appropriate seeing. The finding algorithm was then tested by comparing the number of detected objects by sextractor laying within one seeing disk radius centered on the position of the simulated galaxies. The success rate for the finding algorithm, as it is shown in Figures 2.8 and 2.9 for two of the clusters in the sample, depends on the brightness of the object and on their light-profile. As expected, the detection algorithm works best for point-like objects (filled squares).

The accuracy in the magnitudes is shown in the lower panel of Figures 2.8 and 2.9. These show the magnitude difference between the value reported by sextractor and the total known magnitude of the galaxy. The differences are greater for galaxies with low surface brightness. Most of the galaxies in the clusters should have a magnitude error no larger than $0.3 \mathrm{mag}$ down to $I=24$, especially for the bulge dominated galaxies.

\subsection{Cluster images}

The cluster images in the $I$-band for the clusters in this sample are shown in Figures 2.9a-h. These images show the central regions for each of the cluster fields covering $3.7^{\prime} \times 3.7^{\prime}$, corresponding at about $1.5 \mathrm{Mpc}$ on a side. The overdensity in the central regions is clear in most of the images. 
C4: J1275.15TR

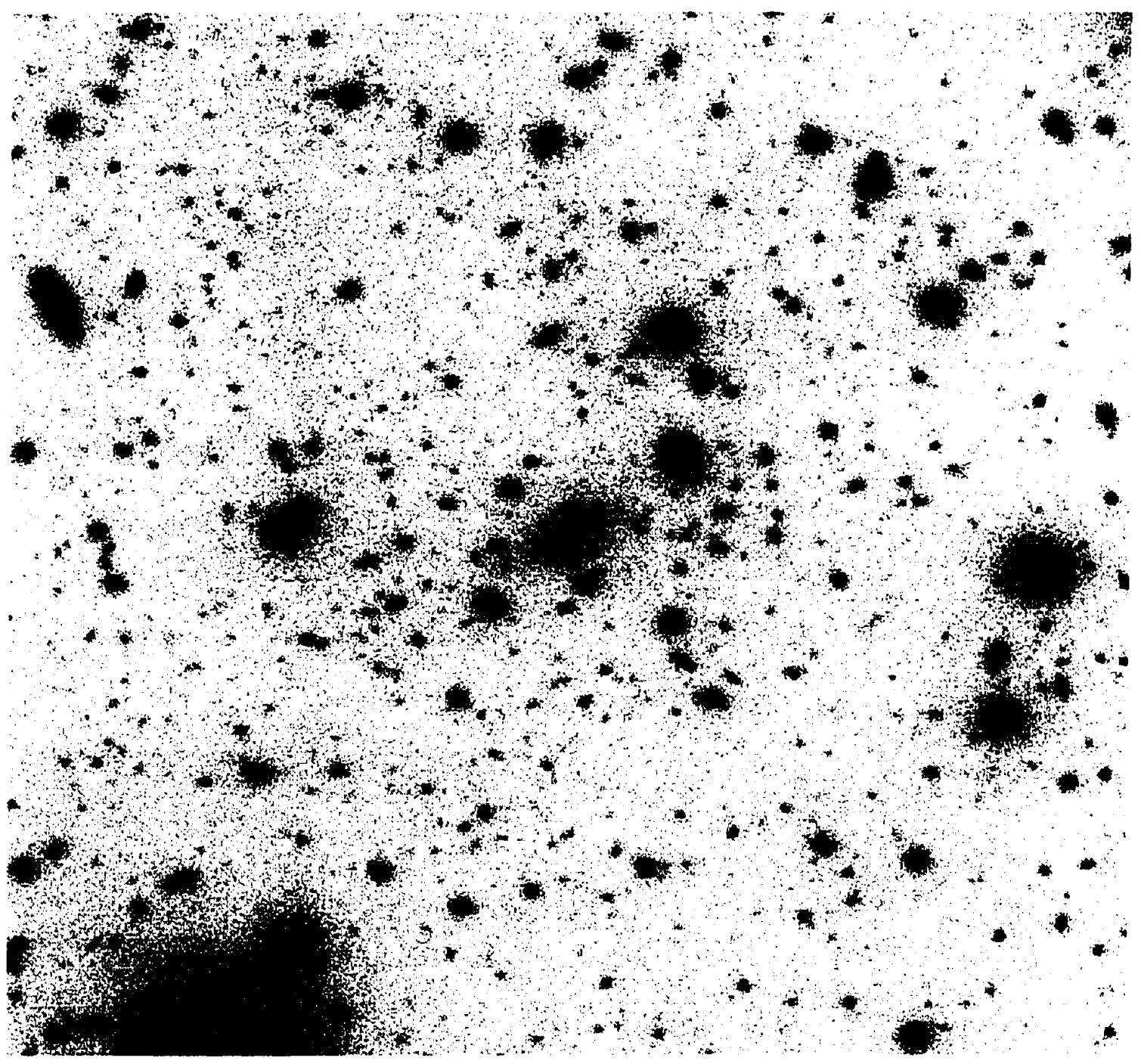

Figure 2.10a: $I$-band image of the cluster J1275.15TR at $z=0.41$. This image is $3.7^{\prime} \times$ 3.7', corresponding at about $1.5 \mathrm{Mpc}$ on a side. North is up and east is at the left. 
C5: J1556.15BL

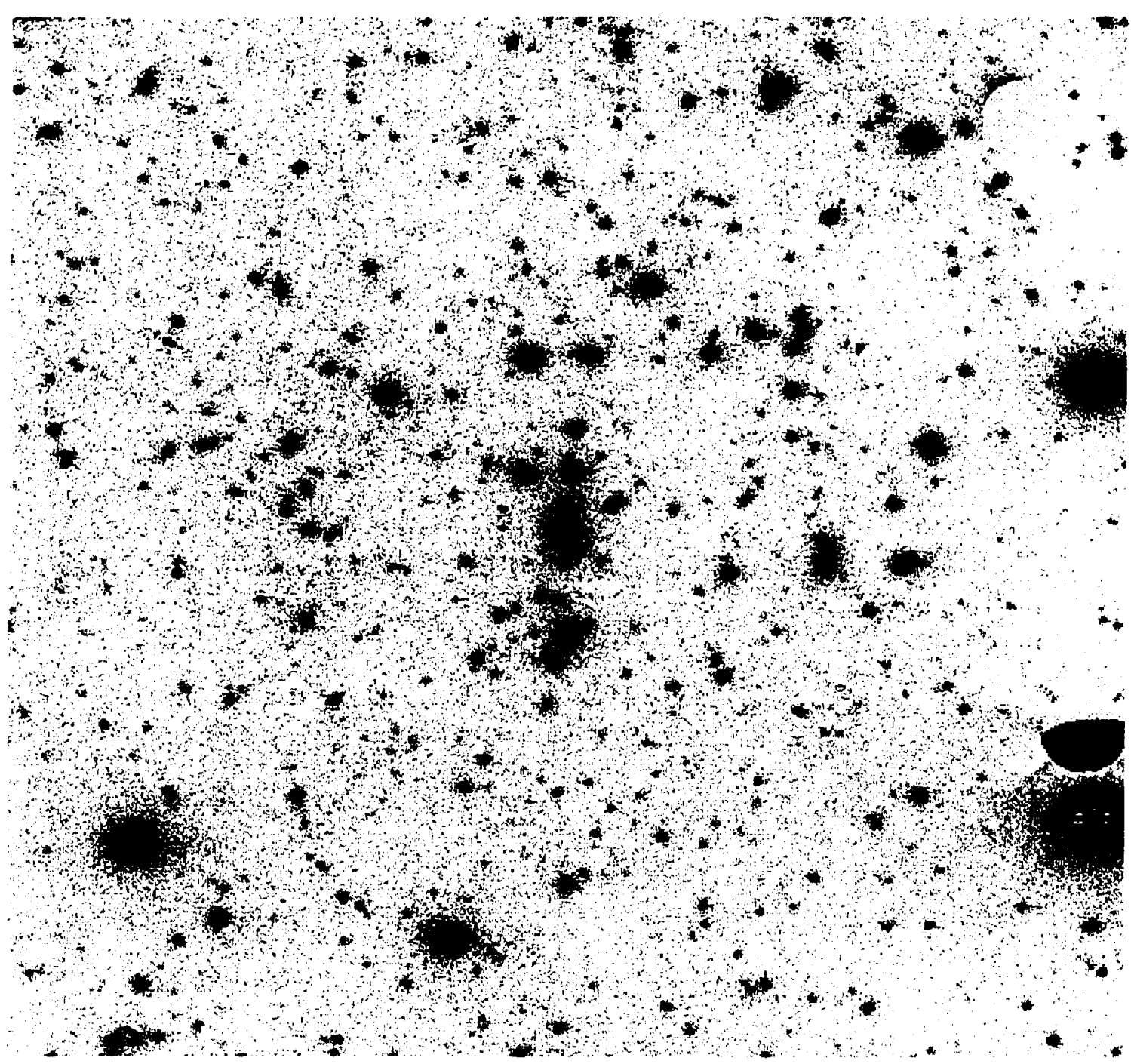

Figure 2.10b: $I$-band image of the cluster J1556.15BL at $z=0.457$. This image is $3.7^{\prime} \times 3.7^{\prime}$, corresponding at about $1.5 \mathrm{Mpc}$ on a side. North is up and east is at the left. 


\section{C6: F1652.20CR}

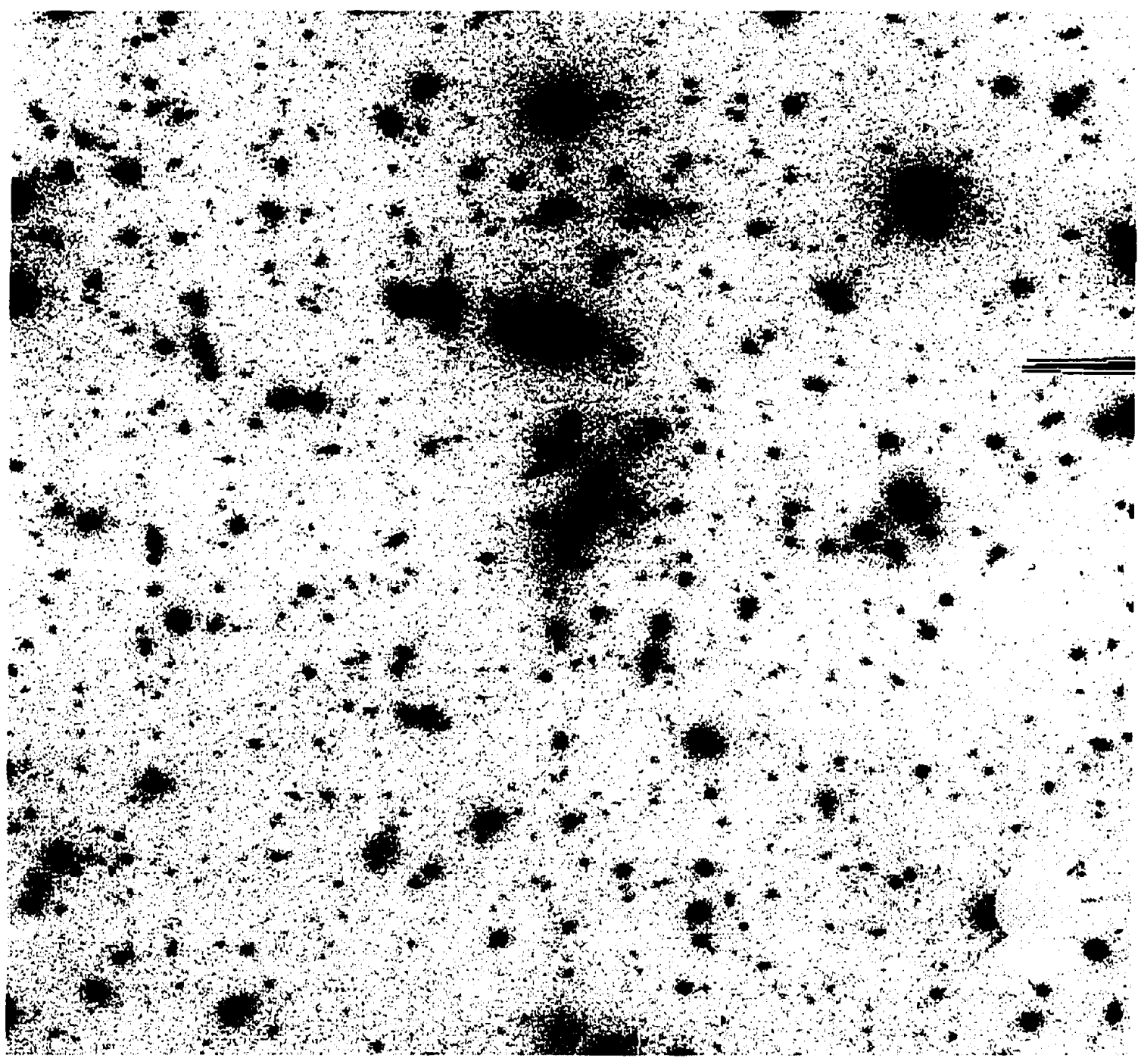

Figure 2.10c: $I$-band image of the cluster F1652.20CR at $z=0.412$. This image is $3.7^{\prime} \times 3.7^{\prime}$, corresponding at about $1.5 \mathrm{Mpc}$ on a side. North is up and east is at the left. 


\section{C8: J2001.21C}

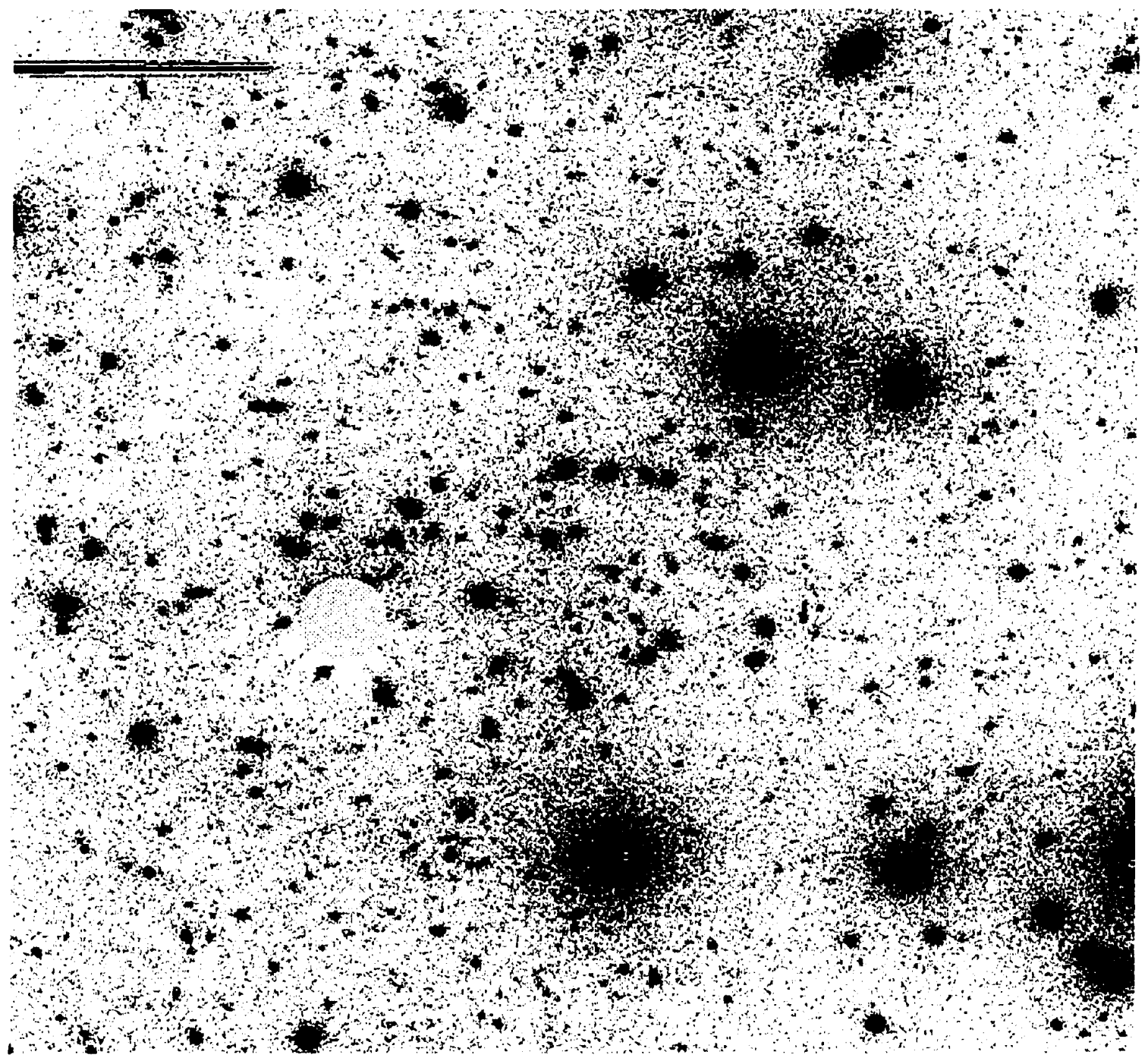

Figure 2.10d: $I$-band image of the cluster J2001.21C at $z=0.413$. This image is $3.7^{\prime} \times$ 3.7', corresponding at about $1.5 \mathrm{Mpc}$ on a side. North is up and east is at the left. 
C11: J1834.8BL

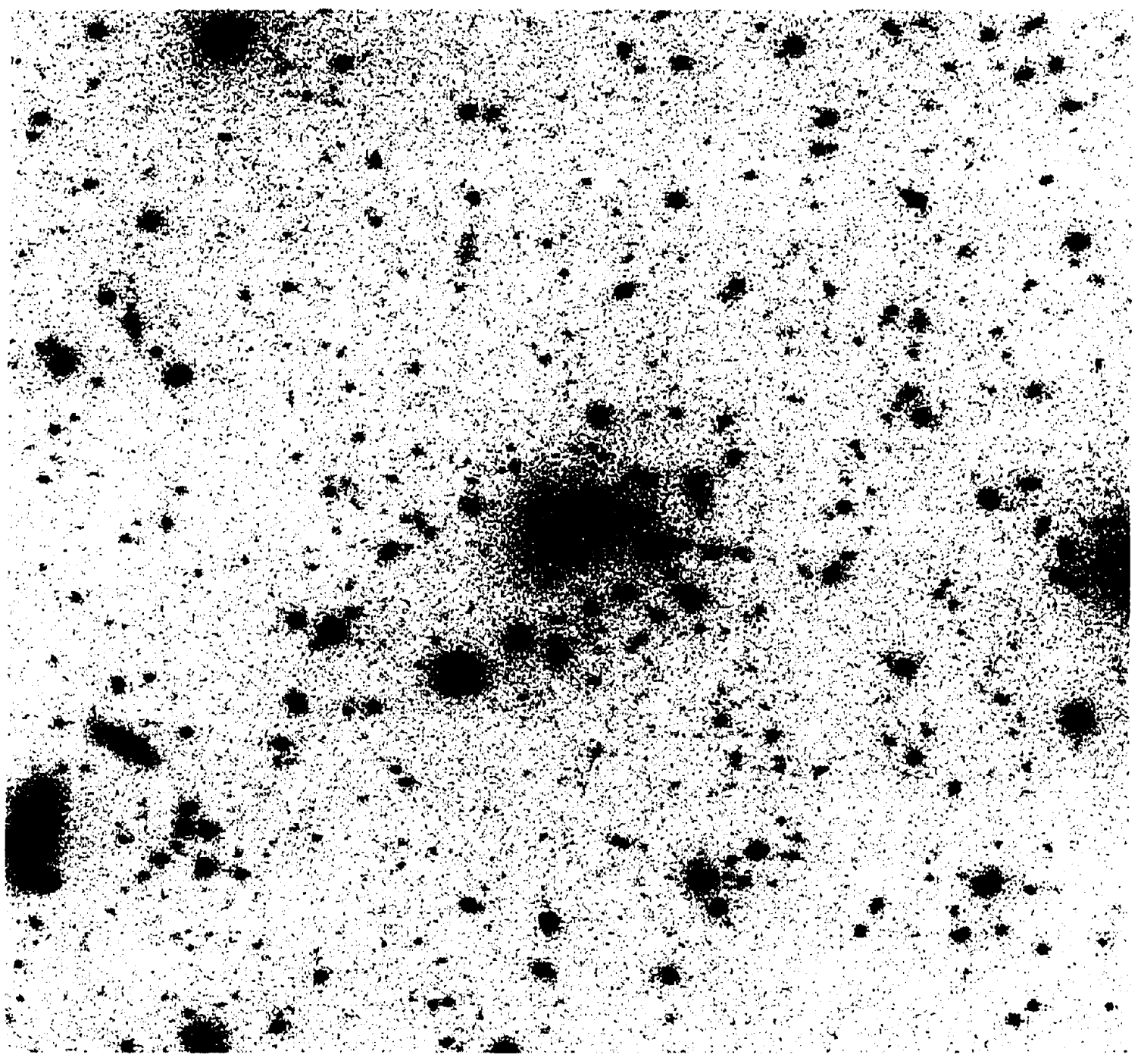

Figure 2.10e: $I$-band image of the cluster JJ1834.8BL at $z=0.445$. This image is $3.7^{\prime} \times 3.7^{\prime}$, corresponding at about $1.5 \mathrm{Mpc}$ on a side. North is up and east is at the left. 


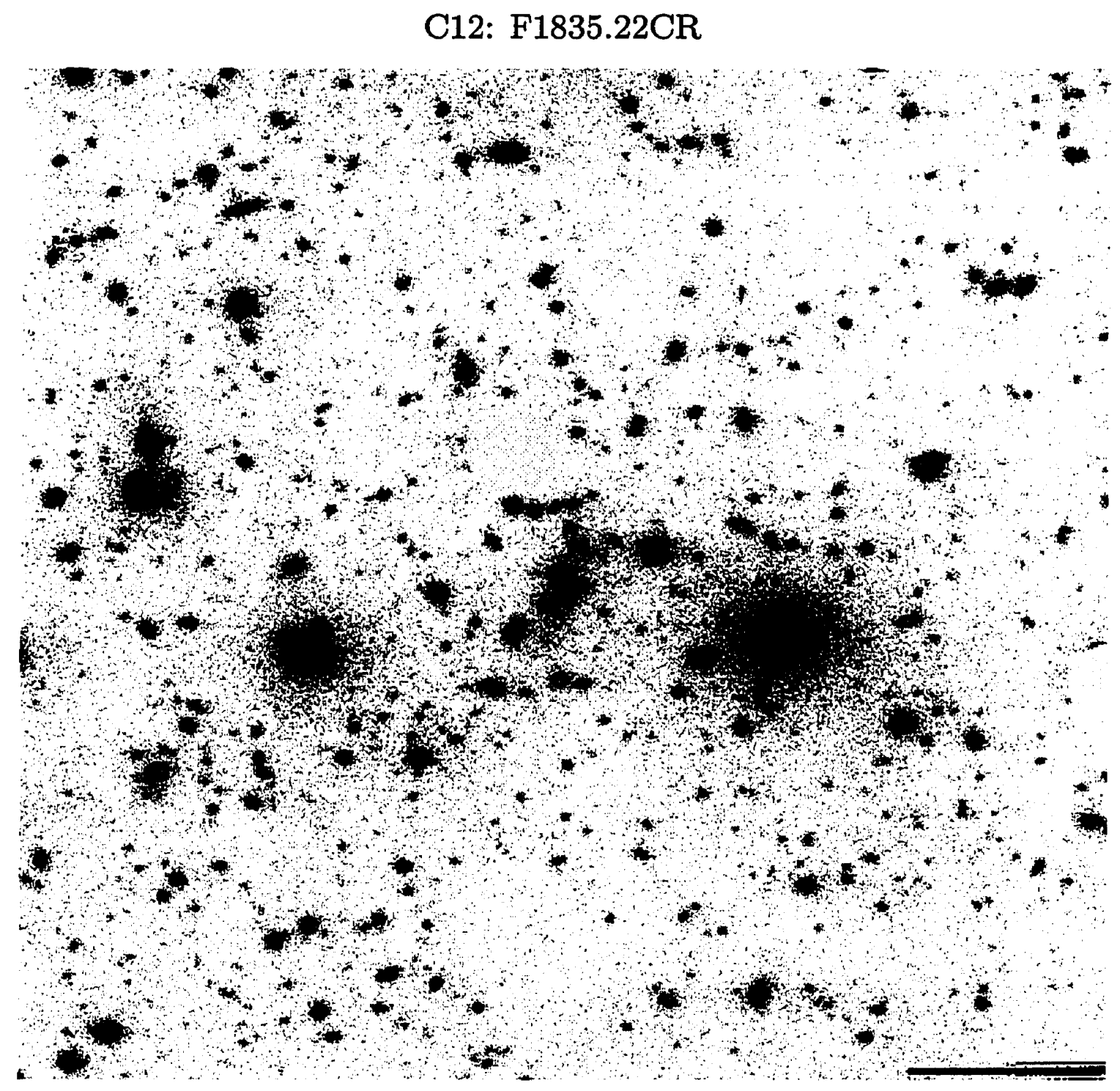

Figure 2.10f: $I$-band image of the cluster F1835.22CR at $z=0.469$. This image is $3.7^{\prime} \times 3.7^{\prime}$, corresponding at about $1.5 \mathrm{Mpc}$ on a side. North is up and east is at the left. 
C15: RXJ1347.5-11.45

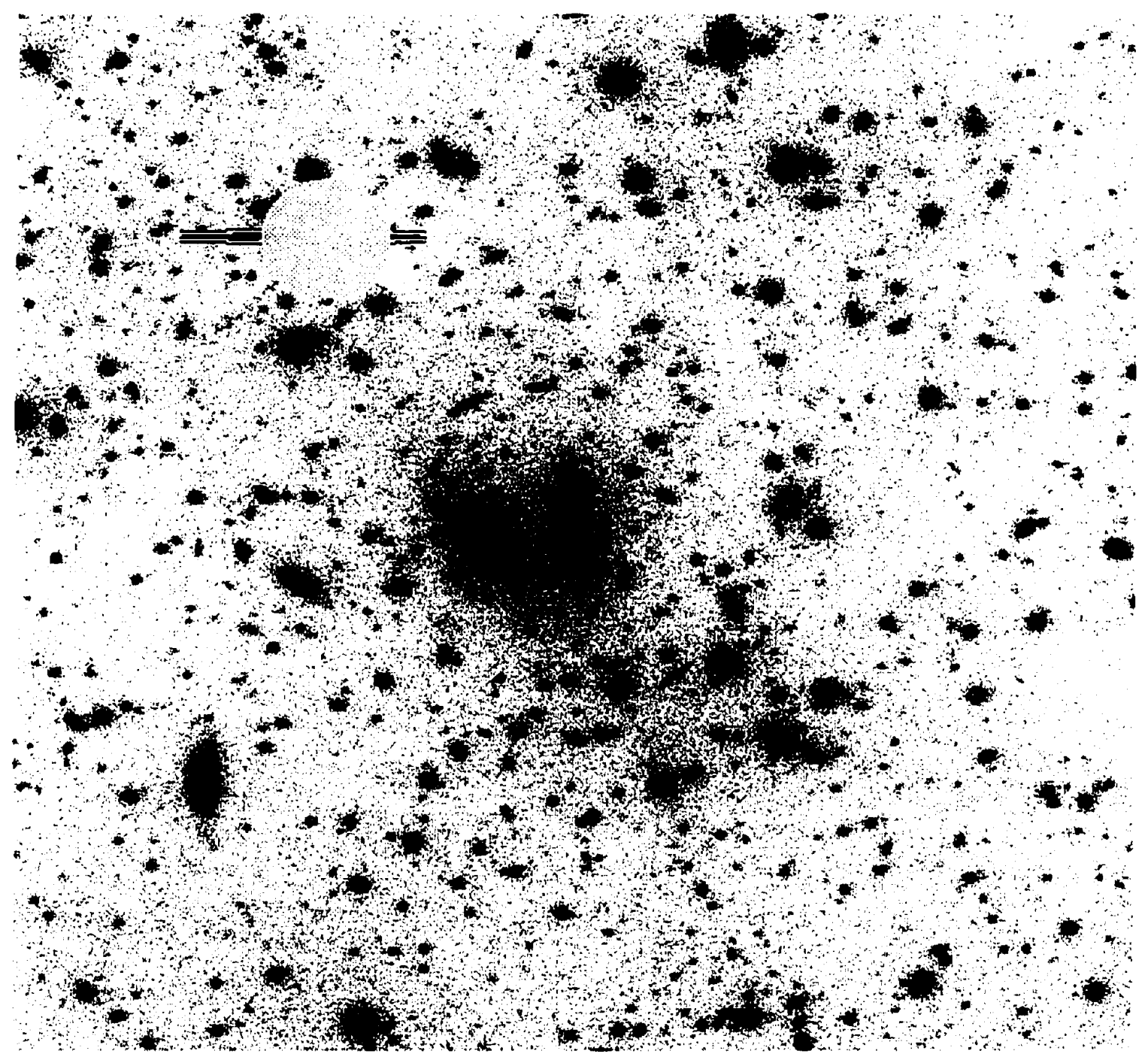

Figure 2.10g: $I$-band image of the cluster RXJ1347.5-11.45 at $z=0.451$. This image is $3.7^{\prime} \times 3.7^{\prime}$, corresponding at about $1.5 \mathrm{Mpc}$ on a side. North is up and east is at the left. 


\section{C19: F1637.23TL}

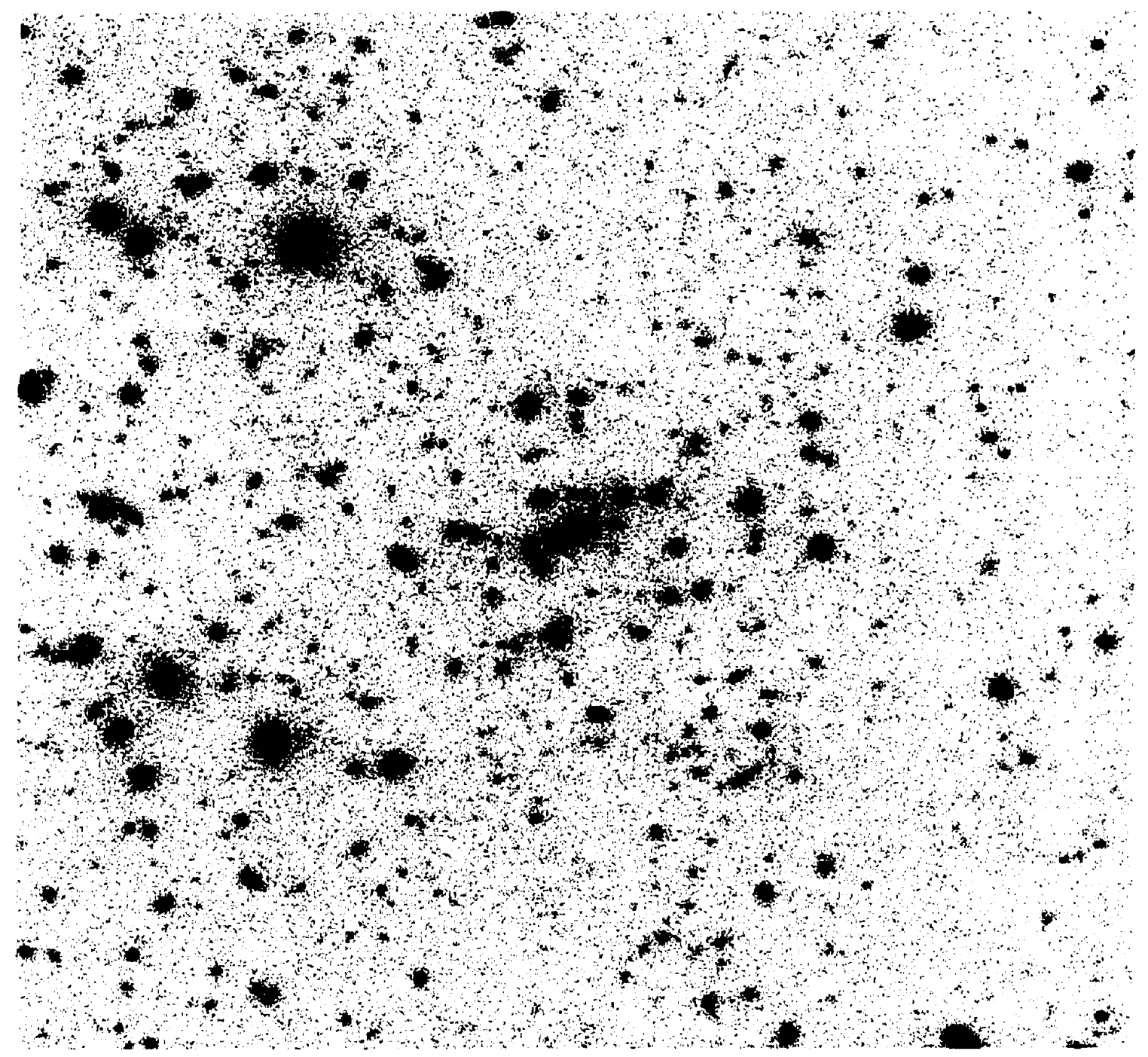

Figure 2.10h: $I$-band image of the cluster F1637.23TL at $z=0.48$. This image is $3.7^{\prime} \times$ $3.7^{\prime}$, corresponding at about $1.5 \mathrm{Mpc}$ on a side. North is up and east is at the left. 


\section{Chapter 3}

\section{Evolution in surface brightness: study of clusters using HST}

Luminosity evolution is an expected consequence of the passive aging of a stellar population such as that believed to make up the bulk of the stars in elliptical galaxies in the central regions of rich clusters. In this chapter I discuss the evidence for such evolution in the cores of clusters of galaxies observed with HST. The work described in this chapter has been done in collaboration with David Schade and Omar LópezCruz, and the results have been published in Barrientos et al. (1996) and Schade et al. (1997).

\subsection{Observations}

A sample of ten clusters having $0.17<z<1.21$ were obtained from the HST archive using the facilities of the Canadian Astronomy Data Centre. These data are processed and calibrated using a pipeline procedure including the latest calibration files available at the moment of requesting the data. Table 3.1 gives the details of the observations for this cluster sample. For all these cluster observations, the data have been subdivided into multiple exposures that have usually been offset, one from each other, by a small amount. This observing procedure has the double purpose of rejecting cosmic ray detections (very abundant in the HST images) and 
Table 3.1: Clusters selected from the HST archive

\begin{tabular}{lccrccrc}
\hline \multicolumn{1}{c}{ Cluster } & $\boldsymbol{z}$ & $\Delta M_{B}$ & $\mathrm{~N}$ & Filter & $\lambda$ (rest) & Time & $A_{B}$ \\
\hline ABELL 2218 & 0.171 & $+0.19 \pm 0.23$ & 5 & F702W & 5960 & 6500 & 0.08 \\
ABELL 2390 & 0.228 & $-0.34 \pm 0.20$ & 10 & F814W & 6530 & 10500 & 0.32 \\
AC118 & 0.310 & $-0.14 \pm 0.25$ & 7 & F702W & 5330 & 6500 & 0.52 \\
ABELL 370 & 0.373 & $-0.22 \pm 0.19$ & 6 & F814W & 5840 & 10500 & 0.07 \\
CL 0959+4711 & 0.407 & $-0.60 \pm 0.19$ & 13 & F702W & 4944 & 10500 & 0.03 \\
CL 140933+52 & 0.460 & $-0.03 \pm 0.22$ & 4 & F702W & 4780 & 12600 & 0.00 \\
CL 001558+16 & 0.547 & $-0.57 \pm 0.13$ & 28 & F814W & 5180 & 16800 & 0.11 \\
CL 1322+3027 & 0.751 & $-0.75 \pm 0.24$ & 6 & F814W & 4576 & 32000 & 0.00 \\
CL 1603+4313 & 0.895 & $-0.96 \pm 0.22$ & 14 & F814W & 4230 & 32000 & 0.01 \\
3C324 & 1.206 & $-1.21 \pm 0.18$ & 14 & F702W & 3160 & 64800 & 0.13 \\
\hline
\end{tabular}

NOTES. - $N$ gives the number of galaxies with $M_{A B}(B)<-20$ that were used to derive the values of $\Delta M_{B}$ given in this table (adopting $q_{0}=0.5$ ). $A_{B}$ is the $B$-band extinction obtained from the NASA Extragalactic Database (NED). $\lambda$ (rest) is the resframe wavelength (in $\AA$ ), and time corresponds to the total exposure time (in sec) for the observations.

also minimizing cosmetic defects in the CCDs. The combination of the individual frames, to produce the final images to be analyzed, was performed using the tools in the STSDAS package available in IRAF.

At the time of this analysis we did not have a good galaxy photometry package to produce the catalogs, so we constructed them by eye for all the clusters. For the $z>0.5$ clusters we additionally applied a matched-template finding algorithm to detect the objects (a very time consuming algorithm). Elliptical galaxy templates with a variety of sizes, axial ratios, and orientations were convolved with the images of the $z>0.5$ clusters, and a detection significance was computed at each pixel of the image. All the detections with a signal-to-noise ratio greater than 30 were selected and subjected to the fitting procedure explained below. Only the objects in the wide field CCDs were included in the later analysis.

All the selected galaxies were subjected to a "symmetrization" process before performing the light profile fitting procedure. The "symmetrization" procedure helps to primarily deal with other close galaxies that could bias the fitted parameters. This procedure consists of rotating the galaxy image by $180^{\circ}$ and self-subtracting it to produce a new image. This asymmetric image is clipped at the $2 \sigma$ level, leaving only 
the positive $2 \sigma$ deviations (this accounts for the asymmetric components assumed to be positive). The symmetric image is then constructed as the result of subtracting this asymmetric image from its original one. The final symmetric image is then free of companions and ready to be modeled.

The light distributions of the symmetric components were fitted using a code developed by David Schade. This code fits a two-dimensional model (exponential disks and $R^{1 / 4}$ laws) to obtain the best representation of the light distribution of the galaxies. In the code, the models are convolved with an empirically-defined pointspread-function (PSF) and integrated over every pixel in the HST image, which in practice is an image of $128 \times 128$ pixels centered on the targeted galaxy. The PSF is determined using the IRAF package DAOPHOT (Stetson 1987). A total of nine free parameters are determined in these fits: size (scale length for a disk, or effective radius for a bulge), axial ratio, position angle, position and the ratio $B / T$ (ratio of bulge luminosity to the total luminosity of the galaxy). The best fit models are found by minimization of $\chi^{2}$ using a modified Levesberg-Marquadt technique described in Press et al. (1992). The sky level is determined in an annulus around the galaxy and independently from the fittings. The sky is kept fixed through all the fitting procedure.

Three different models were computed and evaluated for each galaxy: a) an exponential disk, b) an $r^{1 / 4}$-law for a bulge, and c) a composite bulge+disk model. Visual inspection of the residuals, i.e. the model subtracted to the original galaxy image - not the symmetric image-, done by David Schade and myself was used to determine the best fit for each of the fitted galaxies. Objects best represented by an $r^{1 / 4}-$ law were labeled as ellipticals and were included in the analysis below. No colour or redshift information was included in the selection of the galaxies. A total of more than 1700 objects were fitted in the fields of these clusters, of which only 238 were classified as ellipticals.

The photometric zero points from the WFPC2 image headers were used to convert the observed counts into flux densities (Whitmore 1995) and then into $A B$ magnitudes (Oke and Gunn 1983). The $A B$ magnitudes were converted to rest-frame $M_{A B}(B)$ 
( $\left.B_{A B}=B-0.17\right)$ luminosities using the interpolation of a present-day elliptical galaxy spectral energy distribution (Coleman et al. 1980) as described by Lilly et al. (1995), and using $H_{0}=50 \mathrm{~km} \mathrm{~s}^{-1} \mathrm{Mpc}^{-1}$ and $q_{0}=0.5$.

\subsection{Local calibration}

The results for the HST clusters were compared to those obtained from the analysis of a local sample of four nearby clusters: A2256, A2029, A957 and Coma (López-Cruz 1997). A very attractive feature of this local sample is that it is similar to the HST sample in many respects:

- The angular resolution for the clusters CL0939+4713 and Coma are 0."18 and $1 . " 6$, these translate into a physical resolution of 1.2 and $1.03 \mathrm{kpc}$, respectively.

- The total linear coverage is similar, being 1.04 and $1.39 \mathrm{Mpc}$ for CL0939+4713 and Coma, respectively.

- The integrated signal-to-noise ratio for a $M_{B}=-21$ galaxy in both clusters is $\sim 100$, i.e. probing down to similar depth into the luminosity function.

- The restframe observations for most of the HST clusters is nearly $B$-band, where the local sample has been observed. This minimizes the uncertainties introduced by the $k$-corrections.

The galaxies in the local clusters of this work were analyzed using the same techniques as the HST clusters, making the local and HST samples of galaxies completely comparable. Thus the local sample provides an excellent anchoring point to study the evolution in the HST sample.

Extinctions corrections in the $B$-band of $0.18 \mathrm{mag}$ for A2256 ( $z=0.0601), 0.07$ mag for A2029 ( $z=0.077), 0.05 \mathrm{mag}$ for Coma $(z=0.023)$, and $0.04 \mathrm{mag}$ for A957 $(z=0.045)$ were obtained from the NASA Extragalactic Database (NED), which provides Burstein and Heiles (1982) values with relative errors in $E(B-V)$ estimated at $0.01 \mathrm{mag}$. When including these corrections, the $M_{B}-\log R_{e}$ relations for these four 


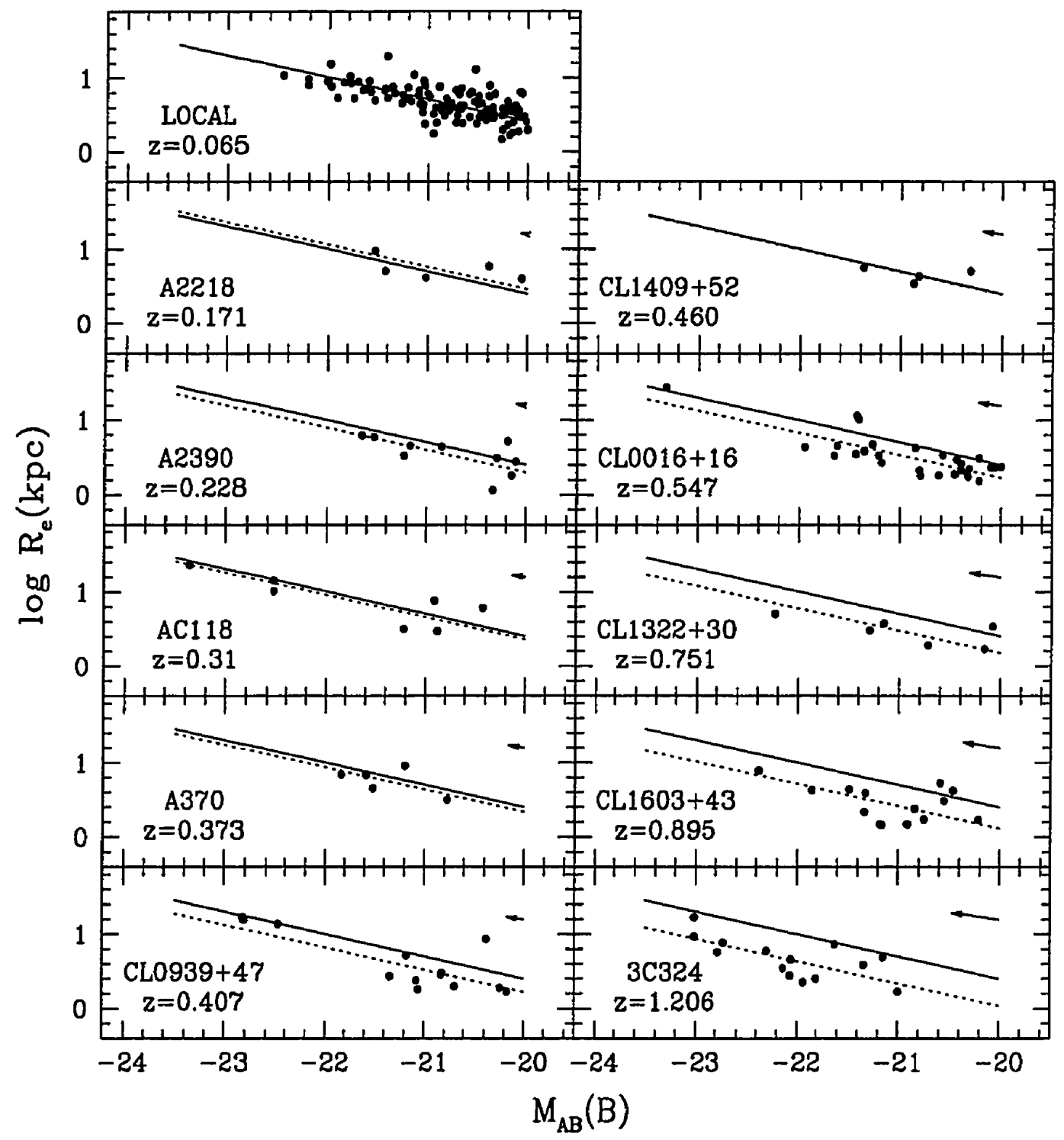

Figure 3.1: Relation between $M_{A B}(B)$ and $\log R_{e}$ for elliptical galaxies in moderate and high-redshift clusters. The solid line in the panels corresponds to the best fit to the local sequence (upper left panel), and the dashed lines indicate the best-fit fixed-slope $\left(\Delta M_{B} / \Delta R_{e}=-3.33\right)$ relation for each cluster. The fits were restricted to those galaxies with $M_{A B}(B)<-20$ in all clusters. The arrow in the upper right corner of the graphs shows the effect of changing $q_{0}=0.5$ to $q_{0}=0.1$. 
clusters are consistent with one another. The offset in luminosity between A2256 and A2029, computed from 38 and 60 elliptical galaxies respectively, is $0.00 \pm 0.10$ mag. These clusters were combined to define the local (size/magnitude) relation for cluster ellipticals. This local relation is shown in the upper left panel of Figure 3.1. The errors for individual galaxies, not shown in this graph, are much smaller than the dispersion in the relation. The solid line, $M_{A B}(B)=-3.33 \log R_{e}-18.65$, corresponds to the adopted local relation obtained from fitting all the ellipticals having $M_{A B}(B)<-20$. This relation is a projection of the fundamental plane (Djorgovski and Davis 1987; Dressler et al. 1987) and it is similar to that of Kormendy (1977), although with the relation in this work having a considerably smaller dispersion.

\subsection{Results}

Figure 3.1 shows the relation between $\log R_{e}$ (half-light radius measured in kpc) and $M_{A B}(B)$ for the elliptical galaxies in the fields of ten HST clusters at $0.17<z<$ 1.21. Luminosities and sizes are calculated assuming that all the elliptical galaxies are cluster members, and using $H_{0}=50 \mathrm{~km} \mathrm{~s}^{-1} \mathrm{Mpc}^{-1}$ and $q_{0}=0.5$. Very little redshift information, if any, is available for the higher redshift clusters.

On average, the $M_{B}-\log R_{e}$ sequences in Figure 3.1 shift toward higher luminosity with increasing redshift, or to smaller sizes if we consider a fixed absolute magnitude value as a reference. These sequences are also consistent with having a single value for their slope, i.e. $\Delta M / \Delta R_{e}=-3.33$ obtained from the local sample. The magnitude shifts (assuming that elliptical galaxies preserve their size and all the change is produced by a change in luminosity), and their corresponding errors were computed using the techniques given in Feigelson and Babu (1992), and also computed according to the robust techniques given by Beers et al. (1990) that are much less sensitive to the presence of outliers. The estimated offsets and errors by using these two different techniques are virtually the same. A change in the slope has a very small effect on the determined offsets, as long as the slope is kept the same for all the clusters. Only those galaxies with $M_{A B}(B)<-20$ were included in the computation 


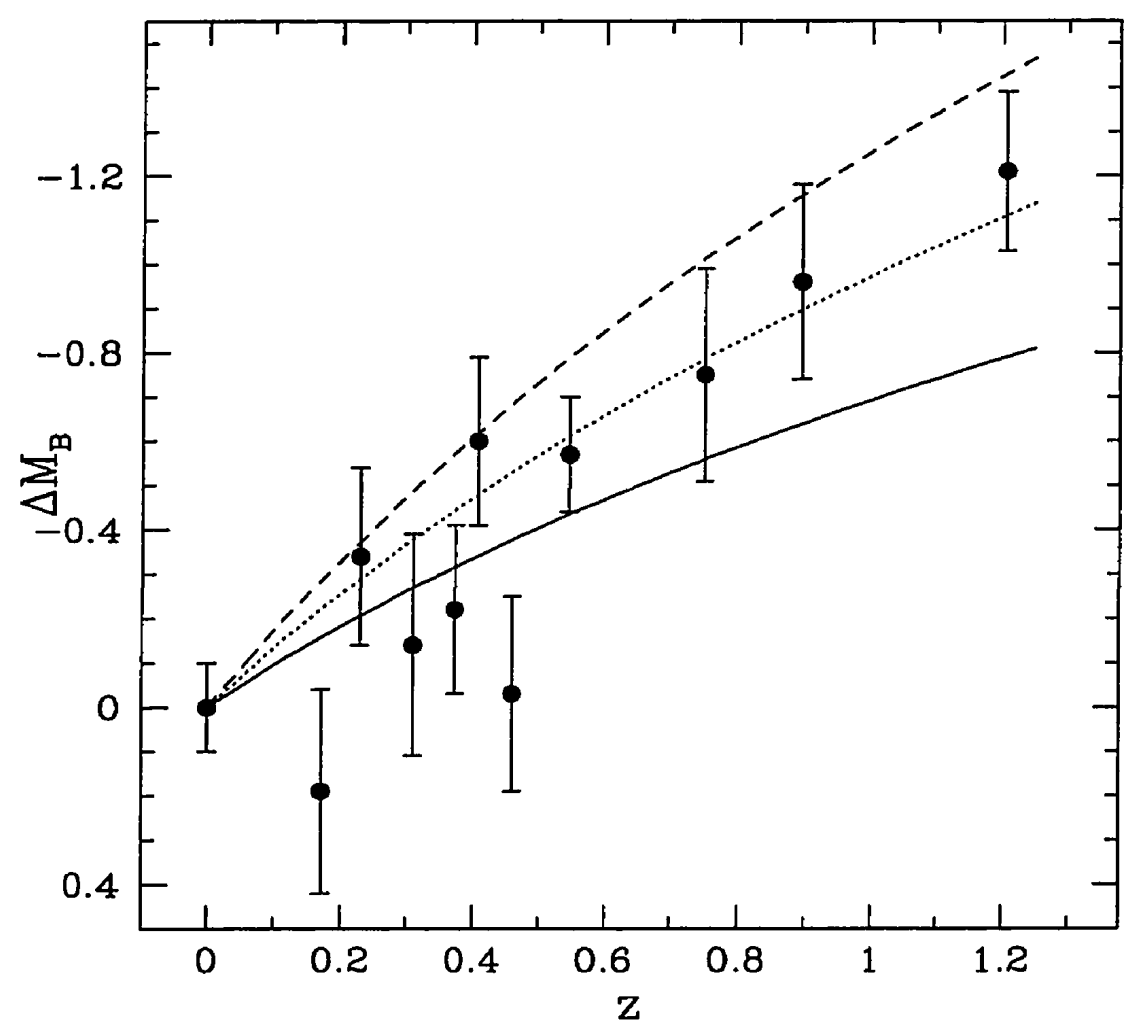

Figure 3.2: Luminosity shift $\Delta M_{B}$ for the HST clusters with respect to the local sample, computed from the $M_{B}-\log R_{e}$ relation. The lines are from the models by Buzzoni (1995) for the IMF power-law indices of $s=3.35$ (solid line), $s=2.35$ (short-dashed line), and $s=1.35$ (long-dashed line).

of $\Delta M$. The computed shifts are presented in Table 3.1, including their estimated errors. These shifts are also plotted in Figure 3.2 as function of the redshift of the cluster.

\section{Observational result \#1}

The observed luminosity evolution in elliptical galaxies from a surface brightness analysis at $z=1.206$ is $\Delta M_{B}=-1.21 \pm 0.18$.

The adoption of a present-day elliptical spectral energy distribution to compute the restframe $B$-band ( $4400 \AA$ ) luminosity results in an underestimate of the $B$-band luminosity of a younger (bluer) stellar population if the observations are at restframe 
longer than $4400 \AA$. For the clusters at $z=0.751$ and $z=0.895$ the observed wavelengths correspond to restframe 4576 and $4230 \AA$, respectively, and therefore the uncertainties in the $k$-corrections are small ( $<0.05 \mathrm{mag}$ ). The situation is more complicated for the cluster 3C324, at $z=1.206$, where the observed wavelength (F702W) corresponds to the ultraviolet (3160 $\AA$ ), making this point much less secure. Dickinson (1995) suggests that these galaxies, although still very red, are $\sim 0.6 \mathrm{mag}$ bluer in $(R-K)$ than a present-day elliptical. The models of Bruzual and Charlot (1993) predict a colour change of only $\sim 0.4 \mathrm{mag}$ from $\tau=5 \mathrm{Gyr}$ to $\tau=15 \mathrm{Gyr}$ (correspondingly roughly to $z=1$ and the present day). Evolutionary corrections would decrease the amount of brightening measured at $z=1.206$.

The absolute magnitudes and the galaxy sizes depend strongly on the adopted values of $q_{0}$ and $\mathrm{H}_{0}$ for the cosmological model, while on the other hand the computed magnitude shifts depend only weakly on them. The effect of changing $q_{0}$ is indicated by the arrows at the upper right corner of the panels in Figure 3.1. These arrows represent the combined changes in size and luminosity that result from changing $q_{0}=0.5$ to $q_{0}=0.1$. The net effect of the change in cosmology, on the computed magnitude shifts, is much reduced by the fact that the total change is almost parallel to the $M_{B}-\log R_{e}$ relation. For $q_{0}=0.1$ stronger evolution would be computed, but only by $-0.06 \mathrm{mag}$ at $z=0.4,-0.09 \mathrm{mag}$ at $z=0.55$, and $-0.17 \mathrm{mag}$ at $z=1.2$. In contrast to the change produced by adopting a different value of $q_{0}$, a change in the Hubble constant has no effect at all in the values of the determined magnitude shifts.

The magnitude shifts, determined from the $M_{B}-\log R_{e}$ relation, are shown in Figure 3.2. These shifts have been computed using the zero point from the local sample from López-Cruz (1997) and assuming that all the observed change in the relation is due to a luminosity effect. In that sense, these magnitude shifts should be understood as the change in luminosity (magnitude) for a galaxy of a given size (half-light radius) when compared to a galaxy of the same size in a local cluster. Superimposed upon Figure 3.2 is the predicted brightening from Buzzoni (1995) for three values of the initial mass function (IMF) and an assumed age of 15 Gyr at the present time. These theoretical tracks correspond to $\Delta M_{V}$ rather than $B$-band 
Table 3.2: Subsequent determinations of luminosity evolution for elliptical galaxies

\begin{tabular}{lcrll}
\hline \hline \multicolumn{1}{c}{ Source } & \multicolumn{1}{c}{ luminosity change } & \multicolumn{1}{c}{ method } \\
\hline Pahre et al. (1996) & 0.41 & $\Delta M_{K}$ & $=-0.36$ & surface brightness \\
Im et al. (1996) & 1.00 & $\Delta M_{B}=-0.5-1.0$ & luminosity function \\
Bender et al. (1996) & 0.37 & $\Delta M_{B}=-0.5$ & fundamental plane \\
Elson et al. (1997) & 0.59 & $\Delta\left(M_{V} / M\right)=-0.44$ & fundamental plane \\
van Dokkum et al. (1998) & 0.83 & $\Delta\left(M_{B} / M^{2}\right)=-0.89$ & fundamental plane \\
Barger et al. (1998) & 0.58 & $\Delta M_{B}=-0.6$ & surface brightness \\
Postman et al. (1998) & 0.90 & $\Delta M_{B}=-1.02$ & BCGs \\
Roche et al. (1998) & 0.90 & $\Delta M_{B}=-0.95$ & surface brightness \\
\hline
\end{tabular}

Different independent determinations of luminosity evolution. This table shows the detected luminosity evolution at the redshift of the study, including the method used.

values, which would be $\sim 0.15 \mathrm{mag}$ larger at $z=1$ (Charlot et al. 1996). If the difference in bands is ignored, then the best-fit IMF slope is $s=2.85 \pm 0.2$ (where the Salpeter value corresponds to $s=2.35$ ).

\subsection{Subsequent confirmation of this result}

The results of this work have been published in Barrientos et al. (1996) and Schade et al. (1997). Other groups using different techniques have confirmed the luminosity evolution presented here, detecting similar evolution in the population of elliptical galaxies in the cores of rich clusters, and in the field ( $\operatorname{Im}$ et al. 1996). Most of these detections have been done using the changes in the fundamental plane when compared to that defined by local ellipticals. These detailed studies have been conducted up to redshift $z=0.9$. A summary of these independent determinations is presented in Table 3.2.

\subsection{Summary}

We have undertaken the study of the elliptical galaxies in the fields of 10 clusters observed by HST, up to $z=1.2$. We modeled the light profile of the galaxies by using an $r^{1 / 4}$-law for bulge dominated systems and exponential disks. Visual classification 
of the residual images, after subtracting the galaxy models, allowed us to segregate the elliptical population for each of the clusters. We found that galaxies up to $z=1.2$ are well represented by an $r^{1 / 4}$-law (just as local ellipticals) and these galaxies follow a well defined relation in the $M_{B}-\log R_{e}$ plane. The relations are all consistent with having a single value for their slopes. These relations shift, on average, towards higher luminosities with increasing redshift (if we assume that the elliptical galaxies in these clusters do not change their sizes from $z=1.2$ to the present), with the galaxies at $z=1.2$ being $\sim 1.2$ mag brighter than their local counterparts, and consistent with models of an old and passively evolving population. The computed magnitude shifts are only weakly dependent on the choice of the cosmological parameters because the effect of changing the cosmology is nearly parallel to the relation in the $M_{B}-\log R_{e}$ plane. The luminosity evolution of the elliptical galaxies detected in this work has been published in Barrientos et al. (1996) and Schade et al. (1997). 


\section{Chapter 4}

\section{Evolution in the luminosity}

\section{function from the CTIO sample}

The luminosity function of galaxies provides a basic description of the galaxy population. It consists in determining the distribution function of the number of galaxies in a given cosmic volume as a function of their luminosity (or magnitude). In the case of the present work, it refers to the galaxies in the central regions of the clusters of galaxies in the CTIO sample.

The galaxy luminosity function can be used to characterize the population of galaxies at two different cosmic epochs, and thus allow for a comparison of their luminosities and/or number density evolution (e.g., Lilly et al. 1995; Sawicki et al. 1997). In the context of this work we will use it to explore the luminosity evolution detected from the surface brightness analysis in Chapter 3. The normalization of the luminosity function depends on the richness of the clusters and therefore cannot be used to study number density evolution of galaxies.

The clusters in the CTIO sample have, in most cases, only a few galaxies with determined redshifts - which usually correspond to the brightest galaxies in the clusters- that have been used to define the redshift of the cluster itself. This lack of membership information for the clusters, and the obvious contamination by field galaxies requires that we apply an statistical method (e.g., López-Cruz 1997) to subtract the contaminants in order to determine their galaxy luminosity functions. This 
statistical method consists of computing the magnitude distribution of galaxies in an aperture centered on the cluster and correcting it by its equivalent field distribution. This correction is essential because the distribution computed on the aperture centered in the cluster includes both the galaxies belonging to the cluster and the field galaxies in the background and foreground of the line of sight to the cluster. The background distribution and the computation of the correction to the magnitude distributions in the clusters fields are explained in detail below.

\subsection{Background correction}

All the clusters in this sample were imaged in $V, I$ and $K$-band, with both optical images covering an area of approximately $5 \times 5 \mathrm{Mpc}$ at the clusters redshifts. To ease the flatfielding procedure (described in Chapter 2) and the estimation of the background contribution, the images were divided into four quadrants placing the centers of each of the clusters in different quadrants (resulting in two per quadrant). This observation strategy increases the areas free of cluster galaxies allowing for an "in field" estimation for the background contamination for all the clusters. We effectively determined the field contamination by including only galaxies that are farther than a predefined radial distance from the center of each cluster. We bin the cluster and field galaxies into 1 magnitude bins in the range $15<I<26$ (in practice we used the data only up to $I=24$ ).

This "in field" background estimation has the advantages of being determined using the same techniques, at the same depth, with the same resolution and not being affected by the observed field-to-field variations in the number of galaxies from different patches in the sky. Although this determination has many advantages, it also has the possible drawback of being contaminated by cluster galaxies that, when subtracted from the cluster counts would produce an over correction to the observed galaxy distribution. This is presumably unlikely to affect the luminosity function. To minimize this effect we need to define the largest area were the cluster galaxies are, without compromising the statistics of the background counts. We found that a good 


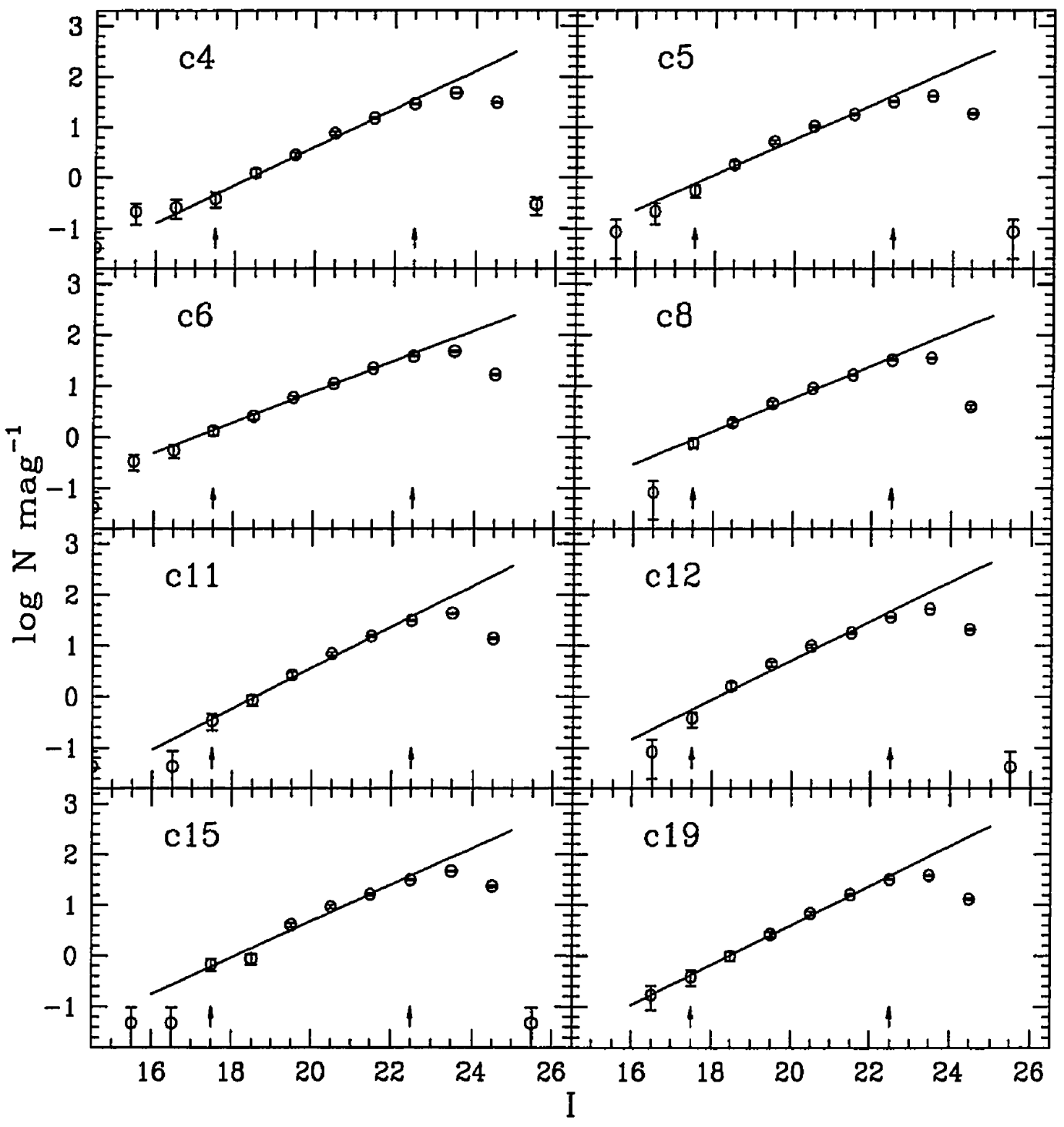

Figure 4.1: Field correction for the clusters in the CTIO sample. This distribution has been computed using all the galaxies in each of the cluster field images, excluding the objects in a circular aperture of $3 \mathrm{Mpc}$ centered on each of the clusters. The numbers of galaxies have been normalized to a $1 \mathrm{Mpc}$ aperture and corrected for incompleteness. The error bars show the gaussian uncertainties for the field counts. The arrows show the range used to compute an "idealized" distribution (shown as a solid line), for each of the cluster fields. 
Table 4.1: Fits to the field counts.

\begin{tabular}{ccccc}
\hline \hline cluster & $m_{I}$ & $z_{p}$ & $\begin{array}{c}\text { total } \\
\text { (uncorrected) }\end{array}$ & $\begin{array}{c}\text { total } \\
\text { (corrected) }\end{array}$ \\
\hline c4 & $0.38 \pm 0.02$ & $-6.95 \pm 0.36$ & 104.9 & 163.6 \\
c5 & $0.35 \pm 0.03$ & $-6.24 \pm 0.56$ & 109.7 & 174.3 \\
c6 & $0.30 \pm 0.01$ & $-5.11 \pm 0.17$ & 130.6 & 173.9 \\
c8 & $0.32 \pm 0.01$ & $-5.71 \pm 0.26$ & 101.7 & 123.5 \\
c11 & $0.40 \pm 0.01$ & $-7.45 \pm 0.29$ & 100.6 & 148.0 \\
c12 & $0.39 \pm 0.03$ & $-6.99 \pm 0.63$ & 123.8 & 252.3 \\
c15 & $0.36 \pm 0.03$ & $-6.51 \pm 0.65$ & 109.6 & 158.0 \\
c19 & $0.39 \pm 0.01$ & $-7.23 \pm 0.23$ & 98.0 & 145.2 \\
\hline
\end{tabular}

$m_{I}$ is the slope of the linear fit for the field counts.

$z_{p}$ is the zero point of the same fit, normalized to an aperture of $1 \mathrm{Mpc}$ diameter at the cluster redshifts.

The last two columns corresponds to the total "contamination" by field galaxies in the cluster aperture in the magnitude range $16<I<24$. These include the raw numbers and those corrected by incompleteness using power-law fit to the number counts. The field contribution mean for all the clusters are $110 \pm 12$ and $167 \pm 38$, for the uncorrected and corrected counts respectively.

compromise to determine the field distribution for these images was to include only those galaxies outside the central $3 \mathrm{Mpc}$ of the cluster images (i.e. a circular aperture of $1.5 \mathrm{Mpc}$ radius). To test if this segregation of field galaxies is sensible we compare the faint galaxy counts with previous determinations.

\subsubsection{Field galaxy counts}

The field galaxy distributions for all the clusters in the CTIO sample are shown in Figure 4.1. These distributions include all the galaxies outside a $3 \mathrm{Mpc}$ aperture centered on the clusters, with no regard for colour information. The counts have been normalized to a circular aperture area of $1 \mathrm{Mpc}$ diameter and have been corrected by incompleteness using the simulations for stars explained in Chapter 2. The errors correspond to the square root of the number of objects in each bin.

We have fitted the field galaxy magnitude distributions by a power law, i.e. a linear fit in the $\log N-I$ diagram, using a least squares technique in the magnitude range $17<I<23$ (delimited by two arrows in Figure 4.1) where the incompleteness 
correction is small. The fits to these distributions are presented in Table 4.1.

The slope of the field counts in the $I$-band ranges from $0.30<m_{I}<0.40$, with a mean value of $0.36 \pm 0.04$ for all the clusters in the sample. These values are slightly higher than the $0.31 \pm 0.01$ and $0.34 \pm 0.01$ values obtained by Steidel and Hamilton (1993) in the $\Re$-band for two areas in the sky. The mean value we found is also higher when compared to the value 0.32 (no errors given) found by Lilly et al. (1991) in the $I$-band, or the $0.331 \pm 0.018$ of Wilson et al. (1997). Although these values are higher when compared to the previous determinations they are not too different either. Two possible explanations for the higher slope observed here could be that: a) the determinations are not completely equivalent because they are not determined in the same magnitude range, Steidel and Hamilton (1993) used $22<\Re_{A B}<26$ and Lilly et al. (1991) at $I_{A B}>23.5$; and b) clusters of galaxies are associated with much larger structures that enhance the number of galaxies at a given redshift, implying that the counts would be higher near the cluster fields. Remember that the field galaxies have been selected from the same images as the cluster galaxies, and that at this stage the only difference between a field and a cluster galaxy is the distance from the cluster center.

\subsection{Count distributions of red cluster galaxies}

The count distributions for the cluster galaxies are built in the same way as the field galaxy count distributions described above, although it is necessary to subtract the estimation for the field contamination to keep only the cluster component.

The total contribution to the cluster counts by field galaxies down to $I=24$ is presented in Table 4.1. For the raw counts this amounts to $110 \pm 12$ galaxies. The total number of galaxies, on the other hand, within a $1 \mathrm{Mpc}$ aperture centered on the clusters is $183 \pm 27$. This indicates that there is $\sim 66 \%( \pm 31 \%)$ contamination by field galaxies in this aperture. This fact implies that the luminosity function determinations would be very uncertain, in spite of the good quality of the data judged from the agreement with other faint galaxy counts. Therefore, we have to 

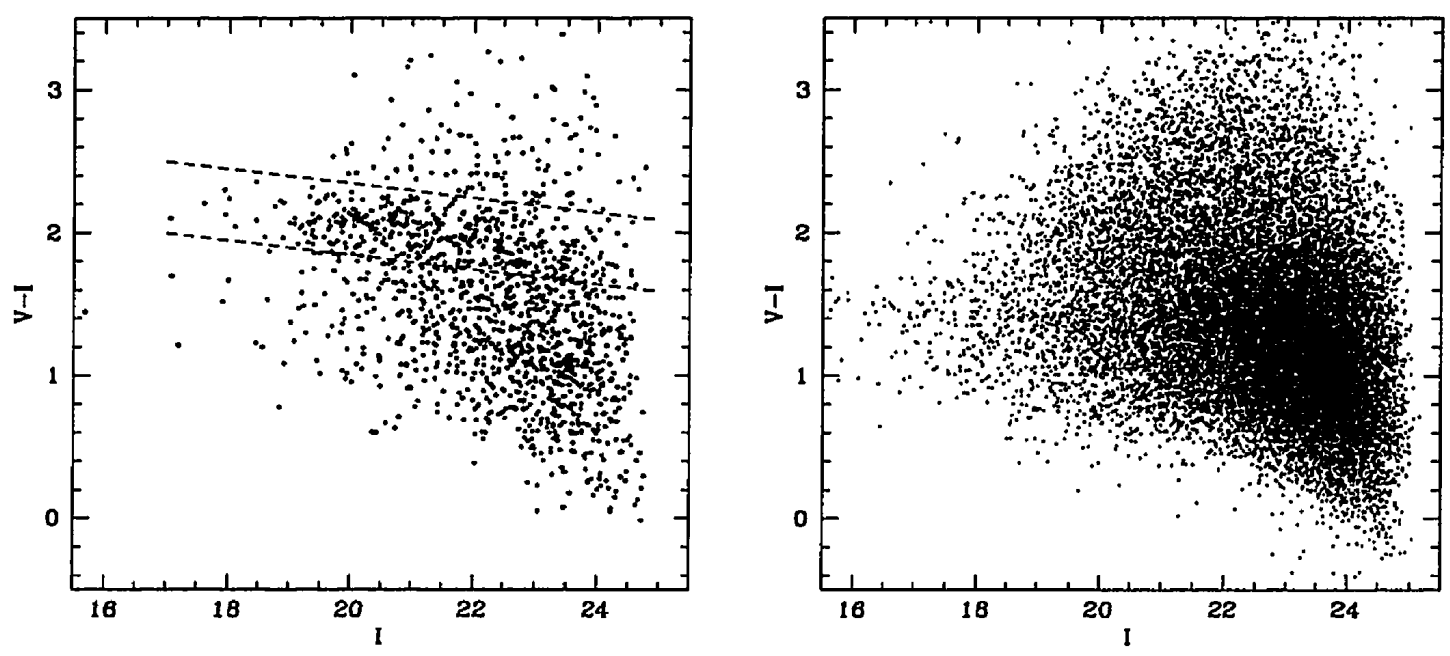

Figure 4.2: Composite colour-magnitude diagram for all the galaxies in the cluster fields. At the left are the galaxies within a $1 \mathrm{Mpc}$ aperture centered on the clusters. The red sequence for the early-type galaxies $(\mathrm{E} / \mathrm{S} 0)$ is at $(V-I) \sim 2$. At the right there are all the field galaxies in all the cluster images. These have been selected by excluding the objects in a $3 \mathrm{Mpc}$ circular aperture centered in each of the clusters.

use an additional selection criteria to enhance the contrast between cluster and field galaxies.

We decided to include only the red galaxies to build the luminosity functions for the clusters in this sample. The colour criteria we used is shown in Figure 4.2, where it is clear that the colour distributions of the red galaxies in these clusters are different than the colours of field galaxies, generally much bluer. The cluster luminosity functions for the red galaxies in these clusters are then less affected by the subtraction of the field component. We included all the galaxies within a band of 0.5 mag in $(V-I)$ centered on the red sequence defined by the early-type galaxies for each of the clusters.

The luminosity functions for these clusters are computed as the difference of the raw binned distributions of the cluster and field galaxies, selected using the same colour criteria, and then corrected by the incompleteness of our catalogs (multiplicative correction). These luminosity functions are shown in Figure 4.3. Each cluster is corrected using the knowledge of the field galaxy distributions in the corresponding 
cluster image. The incompleteness correction applied to these count distributions is determined by assuming that the field faint galaxy counts in each of the images should follow a power-law distribution (the power-law fits computed in the previous section and presented in Table 4.1). This means that the correction applied to the cluster galaxies is the same as the correction necessary to take the observed counts into a power-law for the distribution of the field galaxies for each of the cluster fields. The effect produced by this correction is also shown in Figure 4.3, where the corrected (solid symbols) and uncorrected (open symbols) counts are shown. As expected, the correction only modifies the faint end of the distributions and the change in the counts is only moderate.

One important consideration is that the incompleteness correction might be linked to the light profile of the galaxies that make up the sample. As we have seen in the simulations in Chapter 2, the object detection rate depends on the brightness of the galaxies as well as on the light profile of the objects (such that low surface galaxies are more difficult to detect than point sources for a similar brightness), and therefore the incompleteness correction computed above depends on the morphological mix of the galaxies being considered. As the morphological mix for field galaxies, where the correction is computed, is different than those of the cluster environments applying this correction to the clusters is not strictly correct. This implies that the correction to the cluster counts, obtained from the field counts, should be considered only as a first order correction, i.e. a "better than nothing" correction. A proper correction would require us to have exact knowledge of the light profile distribution of the cluster galaxies.

\subsection{Fitting procedure}

We used the parametric form introduced by Schechter (1976) to model the galaxy luminosity functions for the CTIO sample of clusters. The Schechter function has proven to be a useful empirical description of the observed luminosity function in different environments, while having only a small number of parımeters. The differ- 


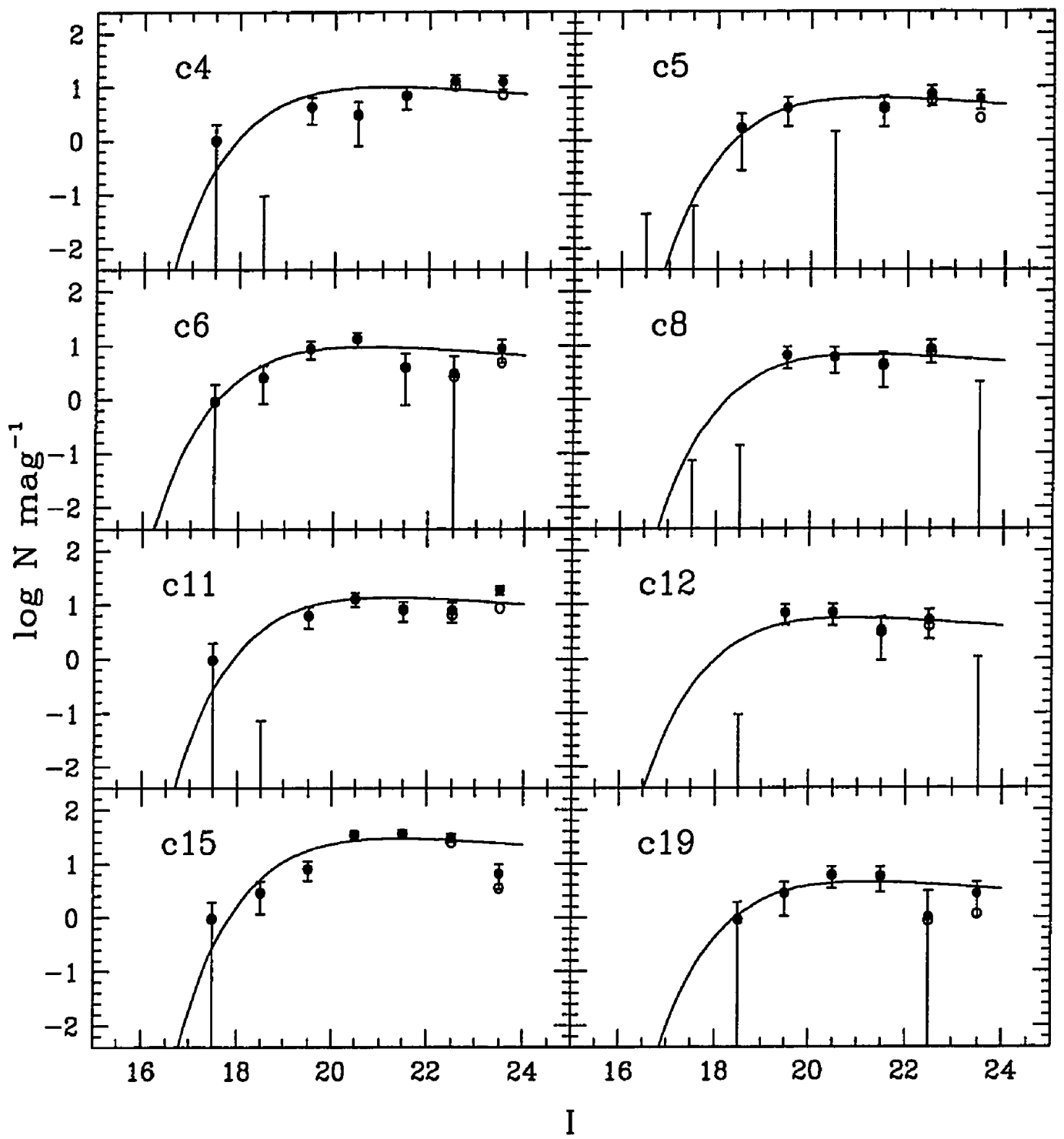

Figure 4.3: Luminosity functions for the red galaxies in the clusters of the CTIO sample. These luminosity functions have been computed in an aperture of $1 \mathrm{Mpc}$ centered on the brightest galaxy in each of the clusters. The red galaxies are defined as the galaxies in a band of 0.5 mag width, in the $(V-I) / I$ colour-magnitude diagram, centered on the red sequence. The open circles show the uncorrected counts, while the filled symbols show the counts corrected by incompleteness. The solid lines show the best fits of a Schechter function, while keeping the faint slope fixed to $\alpha=-0.82$. The error bars are gaussian errors including the uncertainties in the cluster counts and in the background determination. 


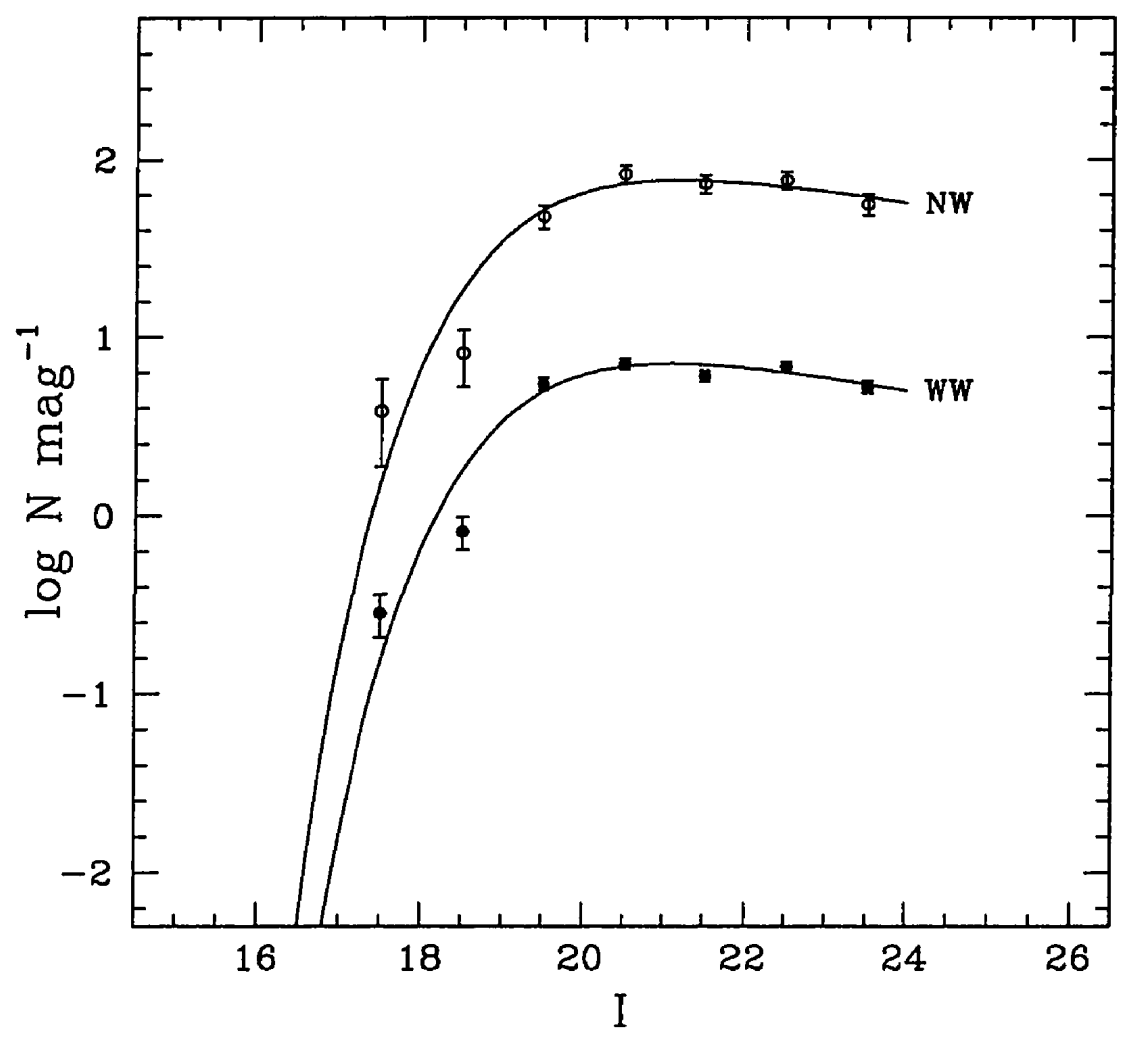

Figure 4.4: Combination of the observed luminosity functions for all the clusters in the CTIO sample. Two different ways of combining the data are shown here: a) a simple addition (labeled as NW; no weighting), and b) a weighted combination, using the inverse of the total number of galaxies as weights (labeled as WW; with weighting).

ential form of the Schechter function, which provides the number of galaxies in the luminosity interval $L$ and $L+d L$, is given by:

$$
n(L) d L=N^{*}\left(\frac{L}{L^{*}}\right)^{\alpha} \exp \left(-\frac{L}{L^{*}}\right) d\left(\frac{L}{L^{*}}\right)
$$

where $L^{*}$ is the characteristic luminosity, $\alpha$ is the faint end slope, and $N^{*}$ is the normalization.

The Schechter function can also be expressed in terms of magnitudes, instead of luminosities, giving now the number of galaxies in the magnitude interval $M$ and $M+d M$. In this case the function takes the form: 


$$
N(M) d M=k N^{*} e^{\left(k\left(M^{*}-M\right)(\alpha+1)\right)} e^{\left(-e^{k\left(M^{*}-M\right)}\right)} d M
$$

where $k=\frac{\ln (10)}{2.5}$, and $M^{*}$ is the characteristic magnitude.

For each cluster luminosity function there are three free parameters to be determined, i.e. $M^{*}, N^{*}$, and $\alpha$. These parameters, providing the model represents the data, define completely the galaxy luminosity function in the observed magnitude range. The number of data points for each cluster in the present sample, however, does not provide enough information to reliably determine these three parameters in an independent way. Therefore, to fit the functional form to the observed luminosity functions we decided to fix the value of the slope at the faint end, and fit for the characteristic magnitude and normalization.

To obtain the best value of the faint end slope we combine all the observed luminosity functions, and then fit all the three parameters. We used two different approaches to construct the combined luminosity function: a) a simple addition of all the individual distributions, and b) an average weighted by the inverse of the total number of galaxies in each of the cluster luminosity functions. The later approach has the purpose of minimizing the effect of the richer clusters in the shape of the final distribution. These two combined luminosity functions, and their respective fits, are shown in Figure 4.4. The determined values for the slope are: $\alpha=-0.82$ and $\alpha=-0.80$ for the unweighted and weighted combinations, respectively.

For this fit, and the following individual fits, we minimize the $\chi^{2}$ statistic as is shown in Equation 4.1. In this equation, $N_{o i}$ and $N_{e i}$ are the observed and expected number of galaxies in the $i$ bin, while $\sigma_{i}$ corresponds to the estimated error in the bin. The expected number of galaxies $\left(N_{e i}\right)$ is computed by integrating numerically the Schechter function in the magnitude range for the corresponding $i$ bin. The error is computed as the square root of the number of cluster galaxies in the $i$ bin (estimated from the Schechter function being fitted) and the square root of the number of background galaxies, added in quadrature. To find the minimum of the $\chi^{2}$ function 
Table 4.2: $I^{*}$ and $N^{*}$ values for the best Schechter function for the clusters of the CTIO sample. These values were obtained keeping $\alpha=-0.82$ fixed through the fitting procedure.

\begin{tabular}{cllllllr}
\hline \hline cluster & $I^{*}$ & $\sigma_{I^{*}}^{-}$ & $\sigma_{I}^{+}$ & $N^{*}$ & $\sigma_{N}^{-}$ & $\sigma_{N}^{+}$ & $\chi^{2}$ \\
\hline c4 & 19.2 & 0.2 & 0.1 & 18 & 2 & 3 & 15.61 \\
c5 & 19.4 & 0.4 & 0.3 & 11 & 2 & 1 & 7.17 \\
c6 & 18.8 & 0.3 & 0.3 & 17 & 2 & 2 & 8.74 \\
c8 & 19.3 & 0.5 & 0.4 & 12 & 2 & 2 & 9.27 \\
c11 & 19.3 & 0.2 & 0.1 & 24 & 2 & 3 & 15.49 \\
c12 & 19.0 & 0.5 & 0.4 & 10 & 1 & 2 & 9.16 \\
c15 & 19.5 & 0.1 & 0.1 & 52 & 4 & 5 & 24.17 \\
c19 & 19.3 & 0.5 & 0.4 & 8 & 1 & 2 & 3.71 \\
\hline
\end{tabular}

$I^{*}$ and $N^{*}$ values for each of the clusters. The errors in each of the parameters have been computed by requiring $\Delta \chi^{2}=1$.

we evaluate it spanning all the possible values of $I^{*}$ and $N^{*}$ (and $\alpha$ for the combined luminosity functions).

$$
\chi^{2}=\sum_{i}\left(\frac{N_{o i}-N_{e i}}{\sigma_{i}}\right)^{2}
$$

This procedure allows us not to only determine the best values for $I^{*}$ and $N^{*}$, but also to define the $1 \sigma$ regions and the confidence intervals for each of the parameters.

\subsection{Results}

The $I^{*}$ and $N^{*}$ values obtained from the $\chi^{2}$ minimization procedure, for all the clusters in the CTIO sample, are presented in Table 4.2. The best $I^{*}$ and $N^{*}$ values and their corresponding $1 \sigma$ regions defined as $\Delta \chi^{2}=2.3$ in the $\left(I^{*}, N^{*}\right)$ plane are shown in Figure 4.5.

As it is expected, richer clusters such as c11 and c15 have very well constrained values for the fitted parameters, while poorer clusters have large uncertainties (particularly in the value of $I^{*}$ ). The best Schechter function fits are also shown in Figure 4.3. This functional form provides a good representation of the observed luminosity functions. 


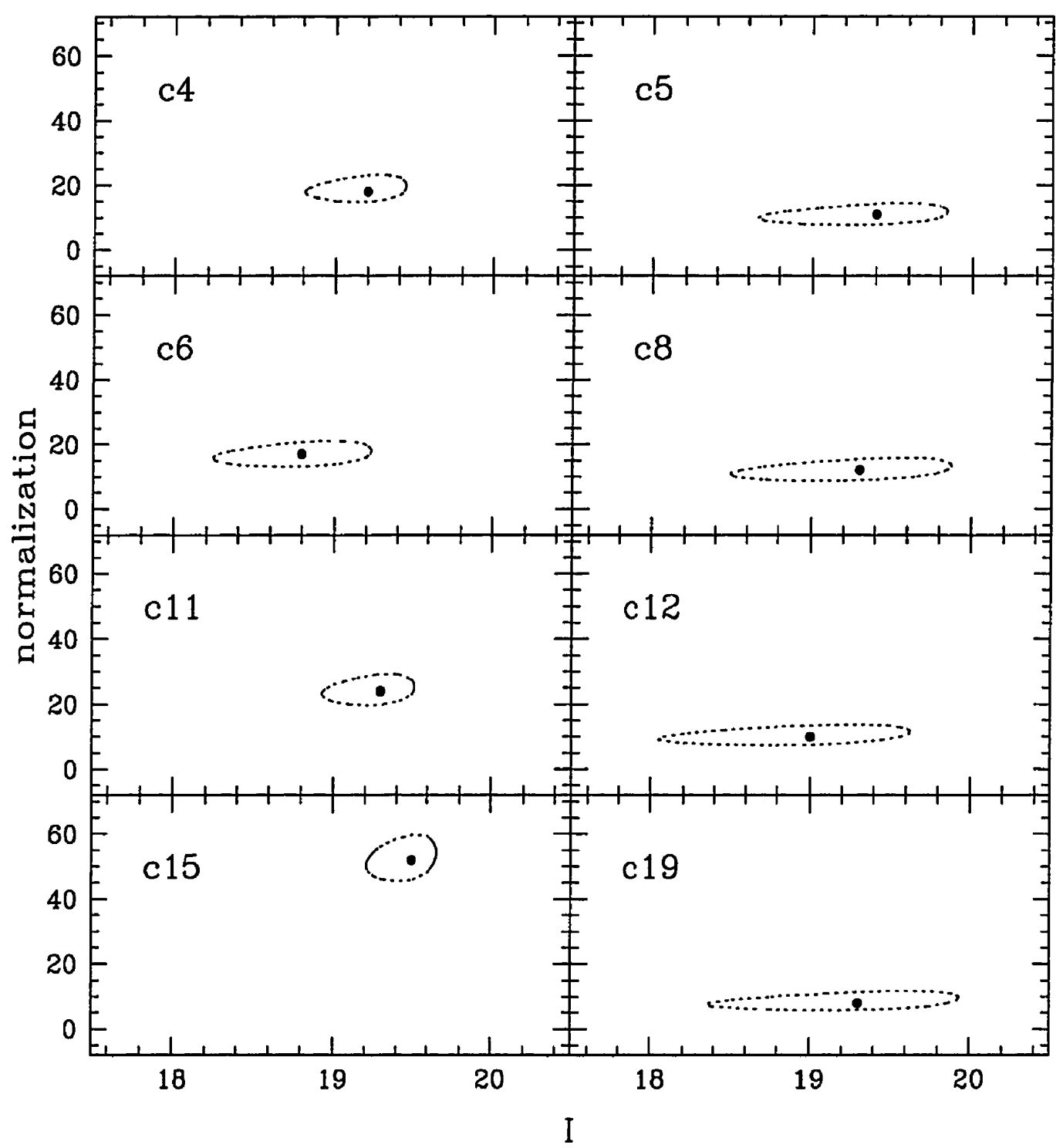

Figure 4.5: Best fit parameters for the Schechter functions for the luminosity functions of the CTIO sample. These fits have been done keeping the faint slope fixed with a value of $\alpha=-0.82$, corresponding the value obtained from the no weighting procedure. The best values for the normalization and the observed $I^{*}$ are showed as a solid dot. The $68 \%$ probability contour is shown as a dotted line. Richer clusters have much lower uncertainty in the value of $I^{*}$. 


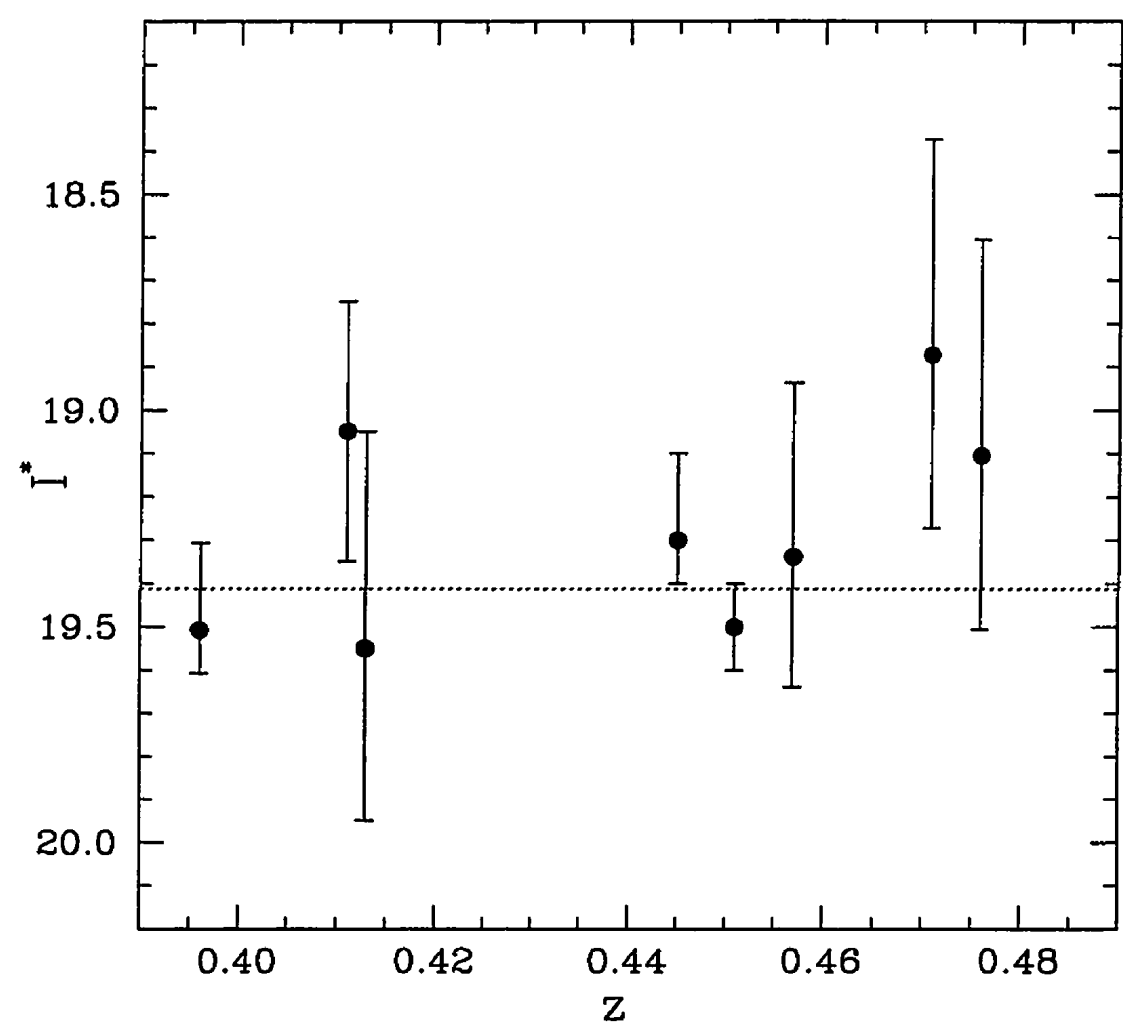

Figure 4.6: $I^{*}$ value from the Schechter function fits as a function of the redshift of the cluster. All the magnitudes have been $k$-corrected to $z=0.45$. The mean weighted average (by the errors) is $I=19.41 \pm 0.19 \mathrm{mag}$, and it is denoted by the broken line. All the data is consistent with having this mean as a single value for $I^{*}$. The error bars denote the $1 \sigma$ uncertainties.

The results of these Schechter functions fits are also presented in Figure 4.6 as a function of the redshift of the clusters. All the $I$-band magnitudes have been $k$ corrected to the value they would have had if all the clusters were at $z=0.45$. These $k$-corrections have been computed by integrating the transmition curve of the $I$-band filter combined with the spectral energy distribution of an elliptical galaxy as given by Coleman et al. (1980), and also including the appropriate bandwidth stretching term. These corrections amount to $\Delta I=0.3 \mathrm{mag}$ at $z=0.40$ and $\Delta I=-0.2 \mathrm{mag}$ at $z=0.48$.

The fits for all the clusters in the sample are consistent with having a single value 
of $I^{*}$. We obtain this value by computing a weighted average of all the determined $I^{*}$ values for the clusters in this sample, obtaining $\left\langle I^{*}\right\rangle=19.41 \pm 0.19$.

In the previous chapter we saw that there is an offset in the size-magnitude plane for the sequence of giant ellipticals in the core of rich clusters observed with HST. We interpreted this change as the result of the luminosity evolution of a passively evolving old population. An interesting question is thus how the computed value of the $I^{*}$ compares to local determinations, and how does this change compare with the luminosity evolution from the surface brightness study?

To determine any luminosity change we must first understand that our $I$-band observations for the clusters at $z=0.45$ are really sampling restframe $V$-band, and therefore we should compare the local determinations to those obtained in the $V$ band. To transform our observed $I^{*}$ into $M_{V}^{*}$ we use the transmition curves of the filters and the spectral energy distribution of an elliptical galaxy (Coleman et al. 1980), taking the precaution that when we transform magnitudes between bands based on the $\alpha$-Lyrae system (with bands having different zero points) we need to transform them first into the $A B$ system (Oke and Gunn 1983). Then, our observed value transforms as:

$$
M_{V}=I_{0.45}+\left(V-I_{0.45}\right)_{A B}+0.46+2.5 \log (1+z)-d M
$$

where $\left(V-I_{0.45}\right)_{A B}=0.04$, and $I_{A B}=I+0.46$ (Fukugita et al. 1995). The bolometric distance modulus from $z=0.45$ to $z=0$ is $d M=42.54$ for $q_{0}=0.1$ and $d M=42.35$ for $q_{0}=0.5$, using a value of $H_{0}=50 \mathrm{~km} \mathrm{~s}^{-1} \mathrm{Mpc}^{-1}$. Although, the choice of the Hubble constant is irrelevant for determining the change in magnitude in the characteristic magnitude, as long it is the same in all studies.

After applying all the transformations, we find $M_{V}^{*}=-22.23 \pm 0.19\left(q_{0}=0.1\right)$ and $M_{V}^{*}=-22.04 \pm 0.19\left(q_{0}=0.5\right)$, for $H_{0}=50 \mathrm{~km} \mathrm{~s}^{-1} \mathrm{Mpc}^{-1}$.

López-Cruz (1997) has determined the luminosity function in the $R$-band using CCD photometry for a sample of 45 relatively local clusters $(0.04 \leq z \leq 0.18)$. Finding a mean value for the characteristic magnitude of $\left\langle M_{R}^{*}\right\rangle=-22.26 \pm 0.29$ 
for his cluster sample, and therefore, we are in position to compare our determination with this value. In order to avoid including extra transformations and their associated errors, we transform our observed $I^{*}$ value at $z=0.45$ directly into restframe $M_{R}^{*}$ using the procedure outlined above.

To correct the observed $I$-band at $z=0.45$ into $M_{R}$ at $z=0$ we use the transmition curves of the filters and the spectral energy distribution of an elliptical galaxy (Coleman et al. 1980). The $k$-correction for the observed $I$-band into local $R$-band is relatively small and well constrained because, as mentioned above, the observed $I$-band corresponds approximately to restframe $V$-band, and therefore the colour correction is small. The total correction is then computed as follows:

$$
M_{R}=I_{0.45}+\left(R-I_{0.45}\right)_{A B}+0.46-0.20+2.5 \log (1+z)-d M
$$

where we use that $R_{A B}=R+0.20$ and $I_{A B}=I+0.46$ (Fukugita et al. 1995). For the spectral energy distribution of the elliptical galaxy (Coleman et al. 1980) we find $\left(R-I_{0.45}\right)_{A B}=-0.36$, where $I_{0.45}$ is the AB magnitude of the galaxy in the $I$-band when it is observed at $z=0.45$.

With these transformations our $I^{*}$ value translates into $M_{R}^{*}=-22.83$ for $q_{0}=0.1$ and $M_{R}^{*}=-22.64$ for $q_{0}=0.5$. These values when compared to the local clusters from López-Cruz (1997) produce a magnitude difference of $\Delta M_{R}^{*}=-0.57 \pm 0.35\left(q_{0}=0.1\right)$ and $\Delta M_{R}^{*}=-0.38 \pm 0.35\left(q_{0}=0.5\right)$. These values are consistent with the amount of evolution detected from the surface brightness analysis in Chapter 3. Here, we have to note that the photometric procedures used in this reference and in ours are both aimed to determine the total magnitude for the galaxies, correcting the effects of seeing and nearby companions. The galaxy magnitudes in both studies should be, in principle, comparable.

\section{Observational result \#2}

The luminosity evolution between $z=0.45$ and $z=0$ from the luminosity function is $\Delta M_{R}^{*}=-0.57 \pm 0.35\left(q_{0}=0.1\right)$ or $\Delta M_{R}^{*}=-0.38 \pm 0.35$ $\left(q_{0}=0.5\right)$. 
In addition to the comparison with local cluster galaxies from López-Cruz (1997) we can compare our value of $I^{*}$ with other local determinations. Marzke et al. (1998) have obtained the luminosity function, by morphological type, for local field galaxies $(z \leq 0.05)$. They have found $M_{B}^{*}=-19.37 \pm 0.10\left(H_{0}=100 \mathrm{~km} \mathrm{~s}^{-1} \mathrm{Mpc}^{-1}\right)$, or $M_{B}^{*}=-20.88 \pm 0.10$ for $H_{0}=50 \mathrm{~km} \mathrm{~s}^{-1} \mathrm{Mpc}^{-1}$, for the early-type galaxies (E/S0) in their sample. To compare with this value we transform our observed $I$-band magnitudes by using:

$$
M_{B}=I_{0.45}+\left(B-I_{0.45}\right)_{A B}+0.46+0.11+2.5 \log (1+z)-d M
$$

where we are using $B_{A B}=B-0.11, I_{A B}=I+0.46$, and the colour $\left(B-I_{0.45}\right)_{A B}=0.97$ for these elliptical galaxies.

These transformations yield $M_{B}^{*}=-21.19 \pm 0.19$ for $q_{0}=0.1$ and $M_{B}^{*}=-21.00 \pm$ 0.19 for $q_{0}=0.5$. These values when compared to the local determination by Marzke et al. (1998) give a magnitude difference of $\Delta M_{B}=-0.31 \pm 0.21\left(q_{0}=0.1\right)$ and $\Delta M_{B}=-0.12 \pm 0.21\left(q_{0}=0.5\right)$. Although these values are slightly lower than those obtained when compared to local cluster galaxies, they are still consistent with the results from Chapter 3. Another point to keep in mind is that the $I$-band at $z=0.45$ is sampling mainly the restframe $V$-band, and because we are using an old local elliptical to compute the $\left(B-I_{0.45}\right)$ colour we are actually defining an upper limit on the change in luminosity. If the galaxies at $z=0.45$ are bluer than their local counterparts (as we will see in Chapter 6), then they should be brighter (in the $B$-band) than the values we have computed.

Another important consideration is that the two local determinations of the characteristic magnitude used as a reference here, have been determined using a faint end slope $\alpha=-1.0$, slightly different from our $\alpha=-0.82$. Using $\alpha=-1.0$ in the determination of the characteristic magnitude in the clusters of the CTIO sample has the effects of making the $I^{*}$ values a few tenths of a magnitude brighter and increasing their uncertainties. Therefore, changing the faint end slope to $\alpha=-1.0$ would make the evolution slightly larger, but it would also increase its uncertainty. 


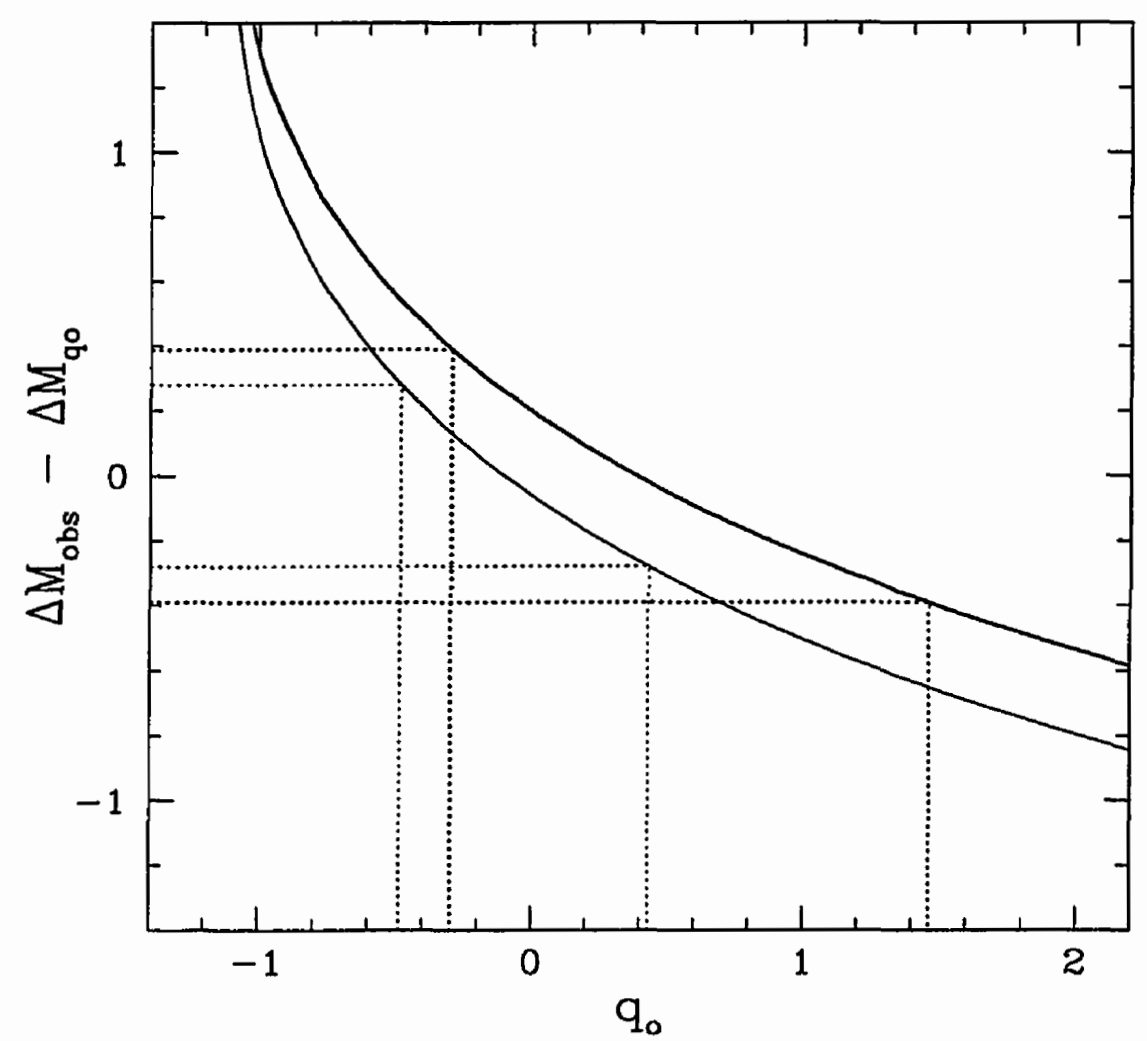

Figure 4.7: Luminosity evolution difference between the surface brightness analysis and the luminosity functions, as a function of $q_{0}$. The luminosity evolution from the luminosity function is computed using as a reference: a) the value for local cluster ellipticals (heavy line, López-Cruz 1997) and b) local field E/S0 galaxies (normal weight line, Marzke et al. 1998). The dotted lines show the range of luminosity evolution and its implication for the range in $q_{0}$.

\subsection{Constraints to $q_{0}$}

From the two independent studies of the luminosity evolution we can set constraints in the value of $q_{0}$. As mentioned before, the evolution obtained from the surface brightness is almost independent of the cosmology because its effect is nearly parallel to the relation we are using to determine the evolution. The magnitude difference between the characteristic magnitude in the luminosity function, on the other hand, depends on the distance modulus, and therefore on the cosmology. Requiring that the difference in luminosity seen in the luminosity function matches to 
that observed from the surface brightness analysis, up to $z=0.45$, gives an estimate of $q_{0}$. This comparison is shown in Figure 4.7, where the uncertainties in the evolution gives us the allowed range in the value of $q_{0}$. From this analysis we obtain $q_{0}=0.38_{-0.68}^{+1.08}$ when using the local value for cluster ellipticals (López-Cruz 1997), and $q_{0}=-0.10_{-0.38}^{+0.53}$ when compared to local field E/S0 galaxies (Marzke et al. 1998).

In the computation above we compare the observed evolution in the surface brightness directly to that of the luminosity function. This is not strictly correct because the evolution from the surface brightness analysis has been measured in the restframe $B$-band, while the luminosity functions have been determined in restframe $V$-band. Using the correction $\Delta(B-V)=0.06 \mathrm{mag}$ (Bruzual and Charlot 1996), we obtain an evolution $\Delta M_{V}=-0.37 \pm 0.18$, implying $q_{0}=0.52_{-0.73}^{+1.15}$ when compared to López-Cruz (1997), and $q_{0}=0.00_{-0.41}^{+0.57}$ when compared to Marzke et al. (1998).

Although the constraint to the cosmology computed above is not very restrictive, it is in agreement with recent determinations.

\subsection{Summary}

In this chapter we have computed the galaxy luminosity functions of the clusters in the CTIO sample. We have found that the faint galaxy counts of field galaxies agree reasonably well with previous determinations. In order to minimize the effect of field contamination we computed the luminosity function including only the red galaxies in the clusters. These luminosity functions are well represented by a Schechter function with a faint end slope of $\alpha=-0.82$. The characteristic magnitude for all the clusters is consistent with a single value $I^{*}=19.41 \pm 0.19$, corresponding to $M_{V}^{*}=$ $-22.23 \pm 0.19\left(q_{0}=0.1\right)$ and $M_{V}^{*}=-22.04 \pm 0.19\left(q_{0}=0.5\right)$. When compared to local determinations, this appears brighter by a few tenths of a magnitude (depending on the cosmology). This brightening - although with large error bars- is consistent with the difference in magnitude observed for elliptical galaxies in the core of rich clusters through the surface brightness analysis presented in Chapter 3. 


\section{Chapter 5}

\section{Colour Dispersions}

Photometric studies of elliptical galaxies in local clusters of galaxies (Sandage and Visvanathan 1978; Bower et al. 1992a; López-Cruz 1997) have found that these galaxies constitute a remarkably homogeneous population. The intrinsic dispersion in the colours of morphologically classified E/S0 galaxies is only $\delta(U-V) \sim 0.04$ mag for the galaxies in the Coma cluster, and when the S0 galaxies are removed from the sample the small dispersion is completely accounted for by observational uncertainties.

Studies of high redshift cluster ellipticals indicate that these small colour dispersions are in place at even much earlier epochs. Ellis et al. (1997), using HST imaging of three rich clusters at $z=0.54$, found a scatter of $\delta(V-I)<0.1$ mag (restframe $U-V)$ for the ellipticals and S0 galaxies in these clusters. This small scatter corresponds to the galaxies within each cluster as well as the dispersion when the clusters are compared among themselves. This small scatter would push the formation epoch of the stars in these elliptical galaxies up to $z \sim 3$, assuming there is 1 Gyr dispersion in the assembly of these galaxies. Similarly to this study, Standford et al. (1998) using optical and infrared photometry found that the scatter remains small (although with large error bars) and nearly constant up to $z=0.9$.

In this chapter we present an effort to determine the intrinsic dispersion in the colour-magnitude relation for elliptical galaxies in our sample of eight clusters at $z=0.45$. We use this observed dispersion to study the star formation timescale in 
the galaxies within these clusters. Special attention is given to the cluster to cluster dispersion because the star formation history in different clusters should, in principle, be uncorrelated.

Before determining the dispersion and mean colour for the galaxies in each of the clusters it is necessary to define clearly the sample of galaxies being considered while computing these parameters. In order to make the results from this work completely comparable with the local and HST determinations we want to select the galaxies in these clusters based mainly on a morphological criteria. This is certainly challenging for ground based imaging and clusters at $z=0.45$. In addition to this, contamination of such a sample of galaxies is expected to include both galaxies of different morphological types in the cluster itself and galaxies of the general population at different redshifts being in the line of sight to each of the clusters. An effective selection procedure should be able to eliminate both contamination components from the elliptical sample. Membership information in these clusters is scarce, therefore we must include additional complementary constraints to refine the selection of the population of galaxies, in particular the ellipticals, in these clusters. Before discussing these additional criteria in detail we will summarize the membership information already available.

\subsection{Sample selection}

\subsubsection{Spectroscopic redshift sample}

From the eight clusters observed in the present work, seven of them have been selected from the Couch et al. (1991) catalog and the other one from Schindler et al. (1995). These two works typically provide redshift information for a couple of galaxies in each of the clusters. Furthermore, Couch et al. (1991) only published selected spectra for a few of the clusters with no comments on the properties of the galaxies observed. Schindler et al. (1995), on the other hand, presented the spectra for the two dominant galaxies in cluster 15 . 
Bower et al. (1997) performed a dynamical study of a sample of distant clusters, publishing their corresponding redshifts. Their cluster sample includes four clusters in our sample, which obviously increases significantly the membership information of our sample. They provide the individual galaxy redshifts and also flag the galaxies having emission lines (no details about these lines are given) with equivalent width larger than $7 \AA$. For galaxies already observed by Couch et al. (1991) they provide, in most cases, improved redshift determinations from the data taken by Couch et al. (1991).

The current status of the redshifts in the clusters of our sample is summarized below:

Cluster 4 (J2175.15TR) The two redshifts obtained by Couch et al. (1991) have been improved and 17 new redshifts have been added by Bower et al. (1997). Of these new redshifts 8 are cluster members and none of them has been flagged as an emission line galaxy.

Cluster 5 (J1556.15BL) Has only the three redshifts from Couch et al. (1991).

Cluster 6 (F1652.20CR) The two redshifts from Couch et al. (1991) are not modified. 20 new redshifts have been added by Bower et al. (1997), of which 10 are cluster members. Of these 10 members, 3 galaxies have been flagged as emission line galaxies.

Cluster 8 (J2001.21C) Has no additional information, keeping the three redshifts from Couch et al. (1991), although one of these galaxies is not identified in the finding chart. This effectively reduces the number of galaxies with redshifts in this cluster to only two, for the purposes of this work.

Cluster 11 (J1834.8BL) Has two unmodified redshifts from Couch et al. (1991).

Cluster 12 (F1835.22CR) The two redshifts from Couch et al. (1991) remain unmodified. 15 new redshifts have been added by Bower et al. (1997), of which only 7 are cluster members. Of these members, two galaxies have been flagged as emission line galaxies. 


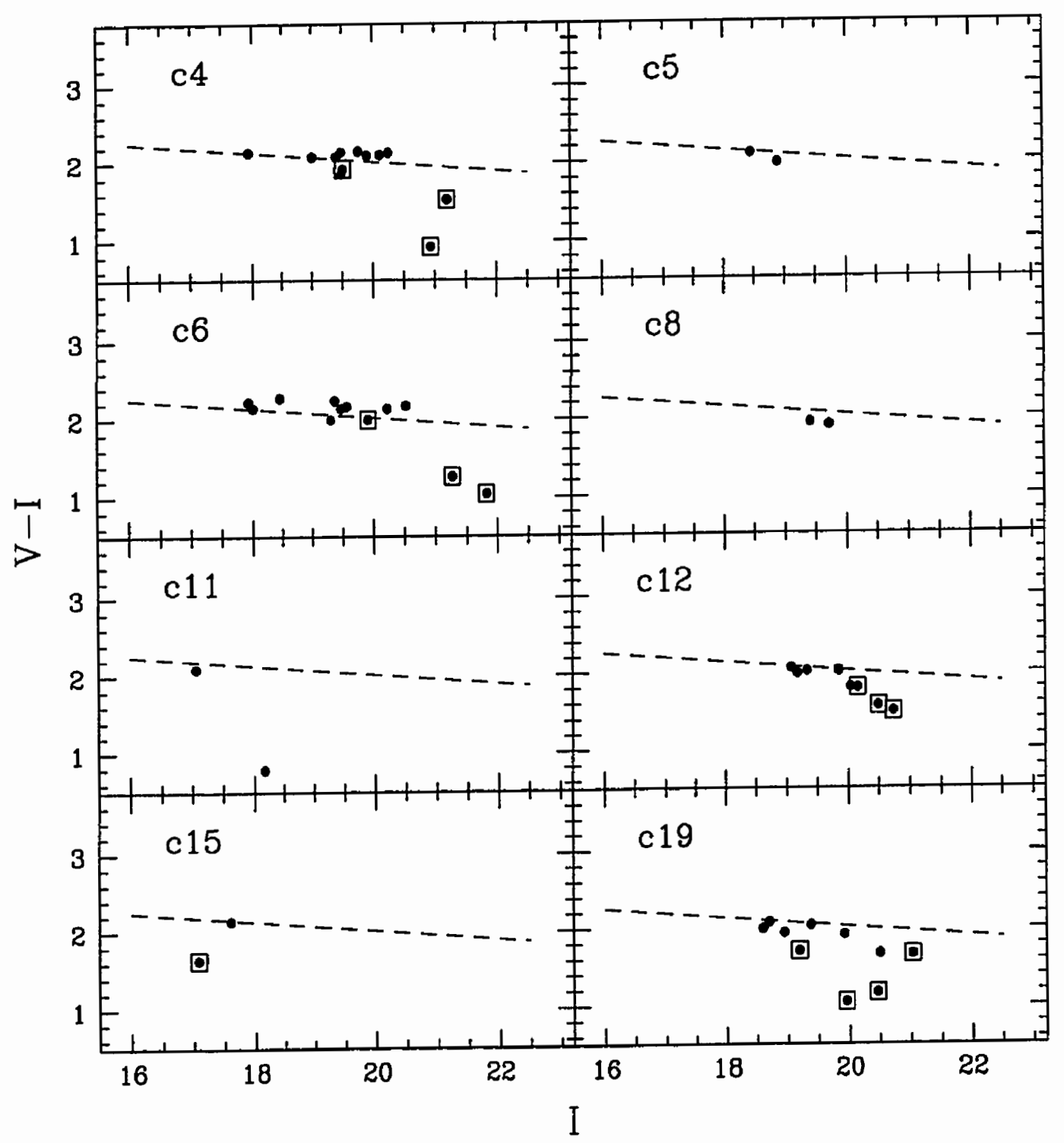

Figure 5.1: Optical colour-magnitude diagram for all the galaxies confirmed as cluster members. Colours have been determined using a fixed 3 arcsec aperture. The photometric errors are shown but they are smaller than the size of the dots. Open squares are galaxies flagged as emission line galaxies and/or for having a disk morphology. The broken line shows the relation for cluster 15. The membership information was compiled from Couch et al. (1991), Schindler et al. (1995) and Bower et al. (1997). 


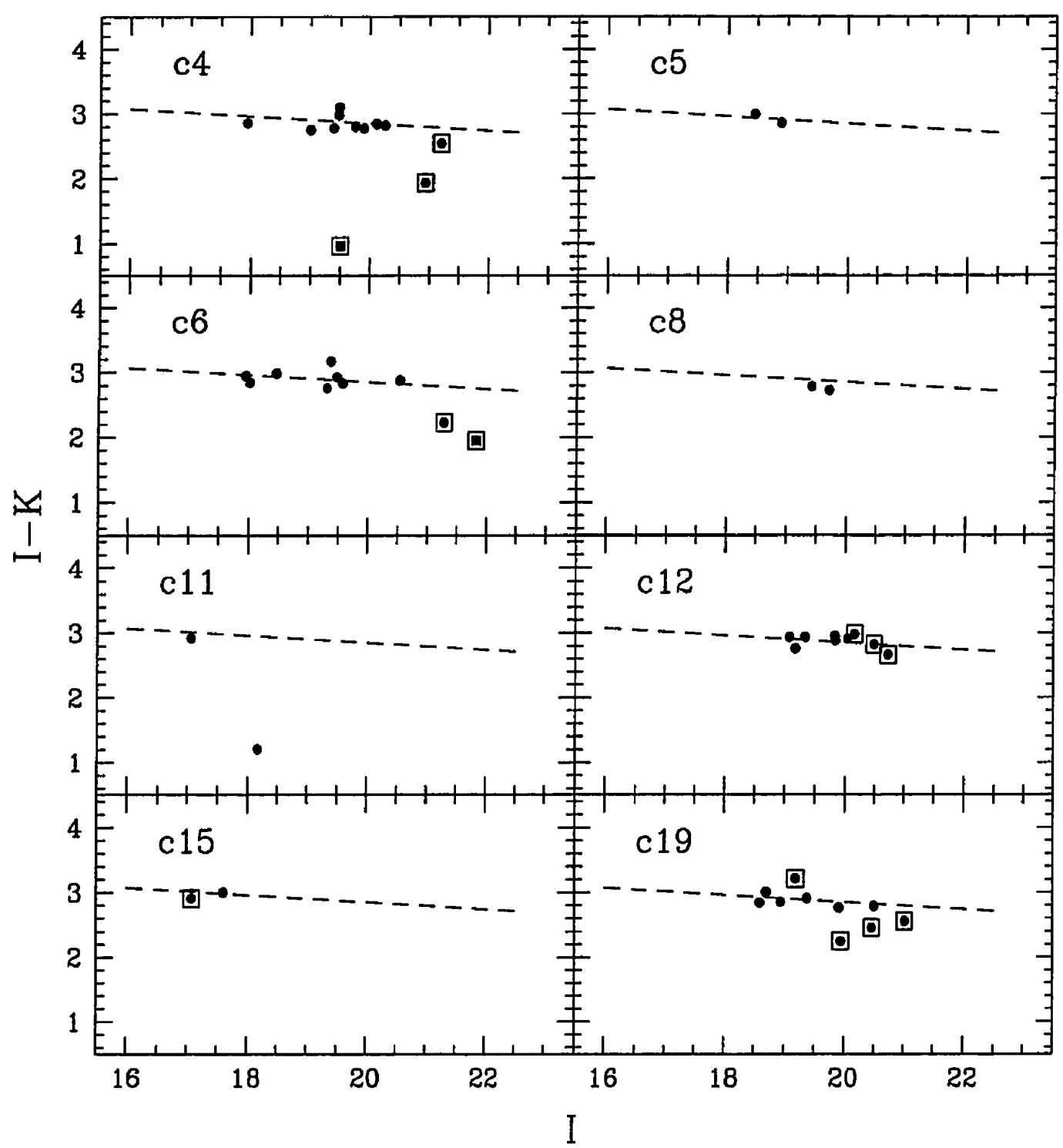

Figure 5.2: Infrared colour-magnitude diagram for all the galaxies confirmed as cluster members. Colours have been determined using a fixed 3 arcsec aperture. The photometric errors are shown but they are smaller than the size of the dots. Open squares are galaxies flagged as emission line galaxies and/or for having a disk morphology. The broken line shows the best fit to the total sample of 69 galaxies in all the clusters. The membership information was compiled from Couch et al. (1991), Schindler et al. (1995) and Bower et al. (1997). 
Cluster 15 (RX1347.5-11.45) The two brightest galaxies have their redshifts measured by Schindler et al. (1995). One of these galaxies clearly shows emission lines in its spectrum, while the other one has only absorption features.

Cluster 19 (F1637.23TL) This is the highest redshift cluster of the sample having three redshifts determined by Couch et al. (1991). These measurements have been revised by Bower et al. (1997). They also added 17 new redshifts in this field, of which 7 are cluster members. A total of 3 galaxies have been flagged as emission line galaxies.

All three studies included in this section provide finding charts for the galaxies with measured redshifts. This makes it possible to identify these galaxies with the objects in our photometric catalogs. The colour magnitude diagrams in the optical and infrared for these confirmed cluster member galaxies are shown in Figures 5.1 and 5.2, respectively. The galaxies generally follow the red sequence, except for those galaxies being flagged as emission line or disk dominated galaxies.

The redshift and emission line information for the galaxies in these clusters is certainly of great value, but it has to be complemented with a morphological classification in order to isolate the population of galaxies we are interested in. A detailed description of how the morphological classification for these cluster galaxies has been performed follows next.

\subsubsection{Morphological sample}

Elliptical galaxies in local clusters are the objects that define the tight sequence observed in the colour-magnitude diagram. Thus, if we want to compare our results with what is observed locally we need to select the galaxies in a similar fashion, i.e. to obtain morphological classification for our sample of galaxies.

Traditional morphological classification of galaxies uses a visual approach to segregate the galaxies into different types. There are several different schemes to classify the population of galaxies. These are usually based on the prominence of a smooth bulge, presence of a disk with or without spiral arms, and the presence of a bar. In 
this scheme an elliptical galaxy has a smooth light profile which lacks a disk and spiral arms. Quantitatively, the light profile of these galaxies is generally well represented by an $r^{1 / 4}$-law.

The visual inspection approach described above is certainly challenging to apply to galaxies located at cosmological distances and observed from the ground under 1 arcsec seeing conditions (representative of the observations in this work). Most of the galaxy features are heavily smoothed by the seeing, making them extremely hard to see. Therefore, if we require morphological information for these galaxies we need to take a different approach, in this case we decided to apply a light profile fitting technique in order to select the elliptical galaxies in these clusters.

This 2-dimensional quantitative approach is based on determining in what proportion a bulge and a disk are present in a given galaxy, with the bulge and the disk being represented by distinctive light profiles (an $r^{1 / 4}$-law for a bulge and an exponential function for a disk). The bulge, disk and bulge+disk models that best represent the galaxy are found by minimizing $\chi^{2}$, where $\chi^{2}$ is constructed from comparing the actual data with the model integrated over each pixel and then convolved with the appropriate seeing ${ }^{1}$. The model that best represents the light profile of the galaxy is then chosen from examining the residuals images created from subtracting the different models from the original data. An example of the different images involved in the fits for these galaxies is shown in Figure 5.1.2.

For the purposes of the present work a classification number in the range 1 to 7 was given to each galaxy after visual inspection of the residuals, according to the following criteria:

1 Bulge Galaxies are classified under this category if their residuals in the bulge subtracted image were clearly smaller than those produced by the disk and bulge+disk models. This classification was also given when the surface brightness of the disk was extremely low in the bulge+disk model, in spite of the fact that the residuals from these models were slightly better than the bulge model

\footnotetext{
${ }^{1} \mathrm{~A}$ more detailed explanation in how the software works is given in Chapter 3
} 
alone. The galaxies in this classification are considered to be ellipticals for the purpose of the present work.

2 Disk Galaxies for which their residuals from the disk subtracted model are smaller than those for the bulge and bulge+disk models belong to this category. Some galaxies having a large low surface brightness bulge component (lower than the disk's) in the bulge+disk model were also classified into this category.

3 B+D Galaxies having their residuals from the bulge+disk model clearly better than those produced from the subtraction of the single component models are included here.

4 Stellar If the residuals produced by all the models are poor, especially if the objects are isolated and there is no apparent reason for the program to fail in producing a good fit, the objects are classified here. The objects in this category generally show a much more concentrated light profile than the models under consideration. Most of these objects are points sources that were slightly outside of our initial criteria to be selected as stars. Only a few objects were classified into this category.

5 Undefined Galaxies for which their residuals do not show clearly that any of the models represent them better than the other models, follow under this category. Fainter galaxies are usually classified as undefined because their low signal-tonoise does not allow us to discriminate the best fit model.

6 Bad fit This category includes either galaxies that are not well represented by any of the fits described above or galaxies having close companions that the symmetrization process did not eliminate.

7 Bad data Misidentification of sources such as spikes near bright stars, galaxies too close to the edges, etc. are included under this category. Only a small fraction of the objects are included here. 

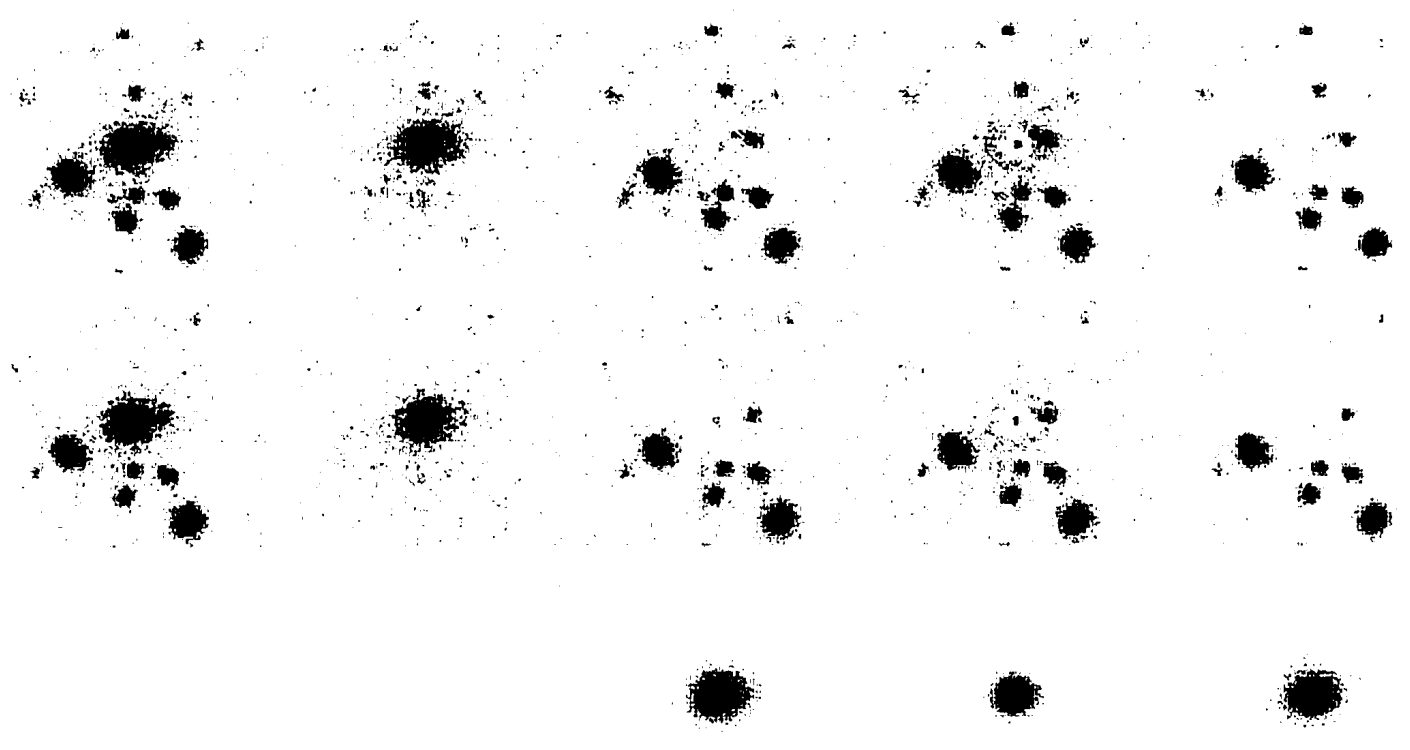

Figure 5.3: Example of the procedure to classify morphologically the galaxies in these clusters. All the fits are performed in an image of $64 \times 64$ pixels centered on the galaxy being fitted. The $I$-band image is shown in the upper left corner and the $V$-band image is shown just below it. Their corresponding symmetrized images are shown in the second column from the left. The residual images, i.e. the subtracted bulge, disk and bulge+disk models are shown in the subsequent columns, always being the $I$-band on the top and the $V$-band in the second line. The actual best individual models are shown in the last two lines, being the bulge, disk and bulge+disk models in the 3rd, 4th and 5th columns, respectively. In the last line the models corresponding to the bulge and the disk in the bulge+disk model are shown. This galaxy has been classified as an elliptical because the $r^{1 / 4}$-law represents best its light profile when compared to the other fits. The disk component in the bulge+disk model is almost non-nexistent. 
A total of approximately 2,000 galaxies (objects within an aperture of $3 \mathrm{Mpc}$ centered on the cluster fields down to $I=22.5$ ) were fitted and visually classified, according to their residuals from the fits, into the categories explained above,

The software to determine these galaxy fits allows us to model the galaxies simultaneously in two bands, and thus to obtain accurate colours for the different components involved. The magnitudes obtained from this procedure include essentially all the galaxy light because the galaxy is usually fitted in a large aperture, and moreover these magnitudes are in principle not affected by seeing. The magnitudes determined using this procedure are also much less affected by crowding, presumably important in clusters of galaxies, than aperture magnitudes because the galaxies are subjected to a symmetrization process before being fitted. The symmetrization procedure consists of rotating the galaxy image in $180^{\circ}$, subtracting it from itself and clipping the negative values at a $2 \sigma$ level, and then subtracting this clipped image from its original image to remove all the non-symmetric components from the image. Note that close companions are also removed by this procedure.

The point spread functions (PSFs) to be included in the galaxy fits were determined empirically using DAOPHOT (available in IRAF v2.10), and individually for all the cluster images, in both optical bands. These PSFs were computed using approximately 50 stars located homogeneously all over the CCD, and they were set to vary linearly along both of the axes of the CCD.

The software to perform the fits was written by David Schade. The interface to use it within the IRAF environment and all the scripts to automatically carry on these fits were written by myself.

\subsubsection{Colour sample}

A total of 50 redshifts are available for the galaxies in the eight clusters of this sample. These redshifts are concentrated in four clusters, leaving the other four clusters with only two redshifts per cluster. From this, it is clear that in addition to the morphological classification for the galaxies in these fields, it is necessary to incorporate another criteria to successfully select the elliptical population within 


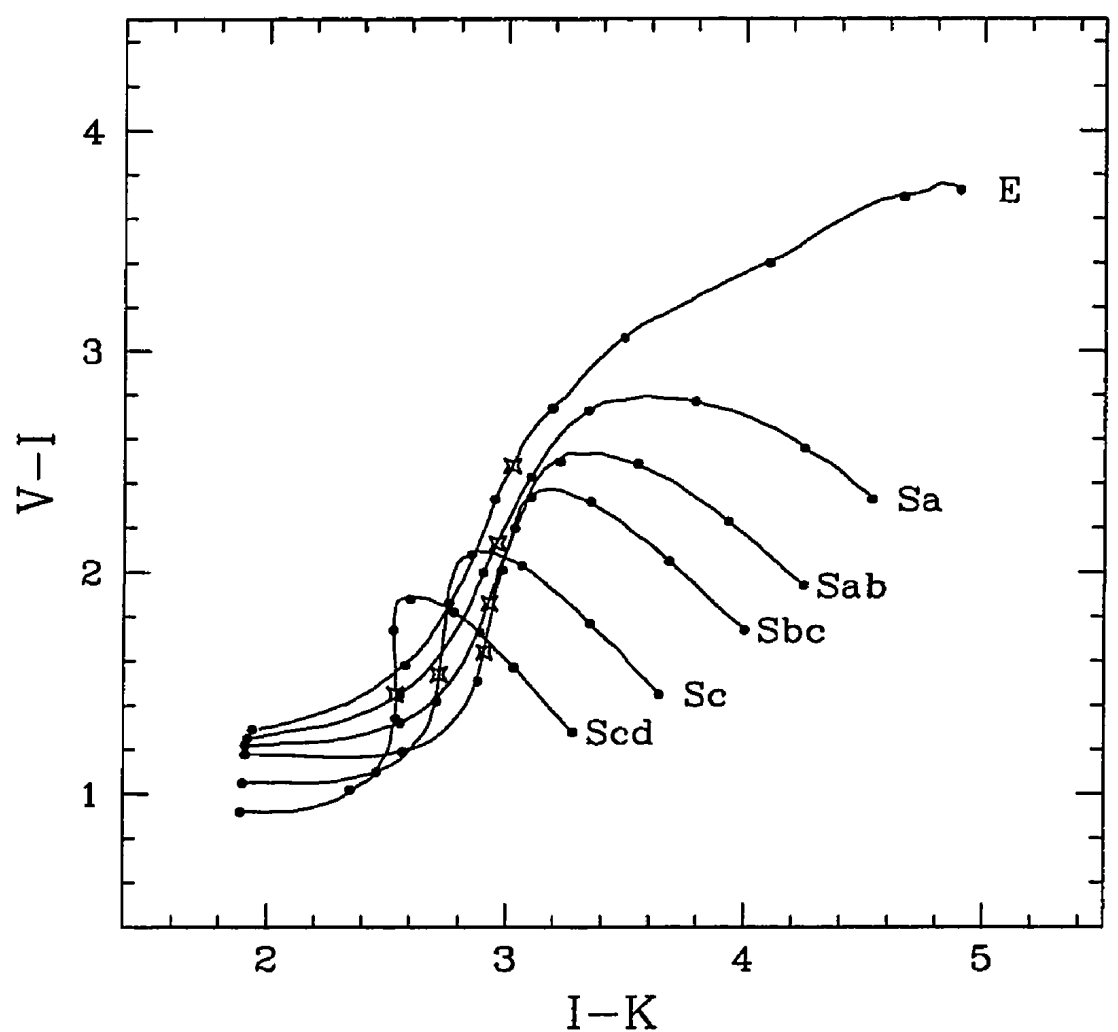

Figure 5.4: Expected colour-colour tracks for galaxies with different SEDs. The solid dots denote the galaxies at different redshifts, from $z=0$ to $z=1.4$ in steps of $\Delta z=0.2$, while the stars denote the galaxies at $z=0.45$. These tracks have been computed using the non-evolving spectral energy distributions from Coleman et al. (1980), extended into the infrared and interpolated to add two intermediate SEDs. When considering only the $(V-I)$ colour to select the elliptical galaxies at $z=0.45$ there is the possibility of contamination by later galaxy types at higher redshifts.

these clusters. This additional criteria should not affect the intrinsic dispersion in the observed $(V-I)$ colour we are trying to determine. Following this line, we decide to incorporate the colour $(I-K)$ as a complementary method to select the red population in these clusters.

While the observed colour $(V-I)$, which translates approximately into restframe $(U-V)$, is sensitive to small amounts of star formation, the observed colour $(I-K)$ remains on the red side of the $4000 \AA$ break, being therefore much less sensitive to any younger population and consequently more dominated by the older stellar population. 


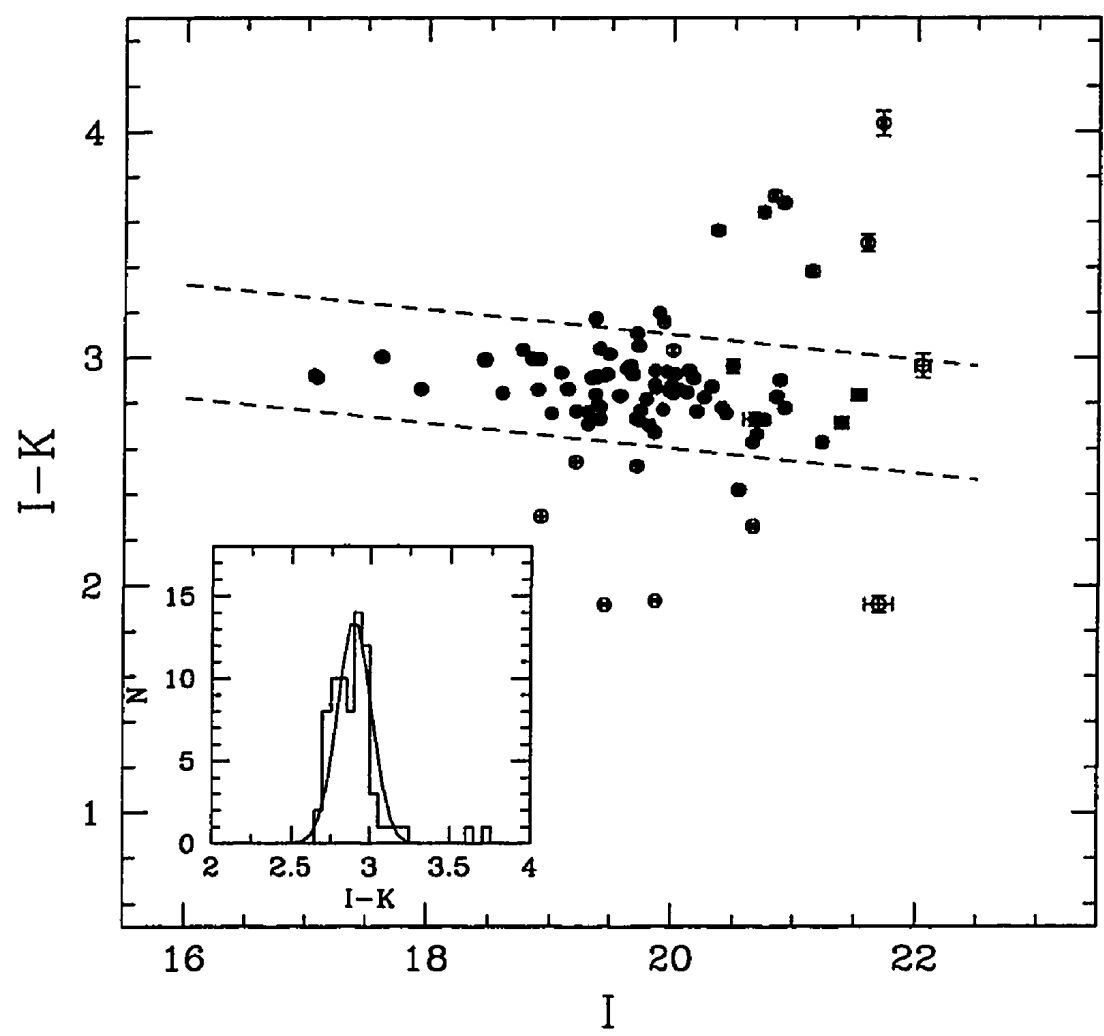

Figure 5.5: Infrared colour-magnitude diagram for all the elliptical galaxies in the central $1 \mathrm{Mpc}$ of the eight clusters. These galaxies have been selected by their morphology alone (open+filled circles -90 galaxies) and for having additionally a $\Delta(V-I) \leq 0.5$ mag from the observed relation in cluster 15 (filled circles -69 galaxies). These galaxies follow a well defined relation with an observed scatter of only 0.107 mag. The broken lines are at $0.25 \mathrm{mag}$ from the best line fit in the $(I-K) / I$ plane and they denote the additional criteria to be applied to isolate the ellipticals in these clusters.

This effect can be easily seen in Figure 5.4 where galaxies of different spectral types at $z \sim 0.45$ (denoted by stars) are spread in about 1 magnitude in $(V-I)$, while the same galaxies only span about $0.5 \mathrm{mag}$ in $(I-K)$. This effect can also be seen within our own data. Galaxies flagged as emission line galaxies in Figures 5.1 and 5.2 are closer to the mean relation in the $(I-K) / I$ plane than in the $(V-I) / I$ one.

Figure 5.4 also shows that galaxies of later types at higher redshifts can potentially contaminate the $(V-I)$ colour distribution if membership is not secure. As an aside, this figure also shows that galaxies dominated by their old populations can be 
segregated from the rest of the population if they are at a high enough redshift, i.e. $z \sim 1$.

To include the $(I-K)$ colour as a constraint to select the elliptical galaxies in these cluster we decided to take an empirical approach. To define this colour criteria we plot all the galaxies selected morphologically as ellipticals in an aperture of 1 Mpc centered on the clusters. These galaxies are shown as filled and open symbols in Figure 5.5. As we are only interested in the galaxies defining the red sequence in the colour magnitude diagram, we select all the galaxies -in all the clusterswithin a band of width $\Delta(V-I)=1$ magnitude around the $(V-I) / I$ sequence for cluster 15 (this is the richest cluster in the sample and its red sequence is very well defined). The galaxies selected using this additional criteria (69 in total) are shown as solid symbols in Figure 5.5. These galaxies are well represented by the linear fit: $(I-K)=-0.055 I+3.951$, with a dispersion of only $\sigma_{I-K}=0.107 \mathrm{mag}$. The distribution of the residuals (corrected to $I=19.4$ ) is shown as an insert in Figure 5.5. The two broken lines define our complementary colour criteria to select the elliptical galaxies in these clusters. The lines are located at $\Delta(I-K)= \pm 0.25$ mag from the linear fit, and they enclose all the galaxies selected morphologically and from the $(V-I)$ relation.

Once the samples of galaxies are clearly defined it is possible to determine the mean colours and dispersion for the galaxies in each of the clusters of the sample.

\subsection{Dispersion within clusters}

The red sequence defined by the elliptical galaxies in these clusters can be characterized by intrinsic colour dispersions and by the colour (at a given magnitude). To determine these parameters we build the final samples using the membership information, the morphological information and/or the $(I-K)$ colour selection, and we fit them using robust statistical techniques. In this section I describe the different samples, the different photometric techniques and the algorithms used. 


\subsubsection{Measurement of colours}

In this work we have determined the galaxy colours using two independent techniques. We have determined them using a fixed 3 arcsec aperture and by using the light profile of the galaxies in the morphology code. In this high precision photometry study these two techniques are affected by different systematics and uncertainties that would have a different impact in the mean colours and in the dispersions. Therefore, before computing the mean colour and dispersions we need to consider:

1. Our fixed 3 arcsec aperture colours are obtained from a much smaller area than the colours from our galaxy profile fitting routine, which are less affected by random errors.

2. The fixed aperture colours have the advantage that their errors have been carefully determined by applying the same techniques, and comparing their magnitudes, to both of the images created from half of the number of images used to produce each final cluster image. This procedure has been done for both optical bands for each of the clusters.

3. The fixed aperture colours have the drawback that they have been determined by using a fixed small aperture and they are of course susceptible to a systematic error product of any mismatch in the seeing of the optical images. We have carefully minimized this effect by convolving the "best seeing" images with a Gaussian kernel to match the "worst seeing" image for each cluster. As we will see later, this procedure clearly helps but it is insufficient to obtain the accuracy required in this work.

4. The colours obtained from the galaxy fits, in spite of being slightly more dominated by random errors, are much more representative of the true colour of the galaxy because they are free of any mismatch in the seeing. These colours are generally measured using a large radii.

5. In contrast to the situation for the errors determined for the fixed aperture colours, the errors in the colours obtained from the galaxy fits are poorly char- 
acterized to this point. Having a good determination of the observational errors is essential because it allows us to constrain the intrinsic colour dispersion for these galaxies.

With all these facts in mind, we decided to use one photometric technique to obtain the mean galaxy colour in each of the clusters, and another to obtain their dispersions. To obtain the mean colour of the galaxy population in each of the clusters we use the colours measured from the galaxy profile fitting algorithm, while for the dispersion around the mean relation we use the fixed 3 arcsec aperture colours. This approach ensures that both of the determinations will use the best available data for it. We also decided to use the "total" I magnitude computed using sextractor to ensure homogeneity in the data, independent of the technique used to determine the colour for each galaxy (the colour differences between one technique and the other are small after all). This also ensures that galaxies for which the profile fits have failed are included as well.

\subsubsection{Estimate of dispersion and mean colour}

Most of the clusters do not seem to be rich enough, and also our photometry do not span a wide enough baseline in magnitude, to determine the slope of the $(V-I) / I$ colour-magnitude relation with confidence (seen Figure 5.6). This is unfortunate because the slope of this relation also contains information about the evolutionary history of these galaxies, as shown by Gladders et al. (1998). We decided to apply the slope measured for cluster 15 to the rest of the sample in order to obtain the zero points and the dispersions. The assumption that all the cluster follow the same relation, i.e. same slope, does not present any real problem because: a) to compare the mean galaxy colours for different clusters it is necessary to adopt a single slope anyway, and b) the dispersion would be affected in the sense that it would increase if we adopt a wrong value, and we would be therefore overestimating it.

We have obtained $(V-I)=-0.059 I+3.195$ using 41 galaxies in cluster 15, selected morphologically as ellipticals and having $\Delta(I-K) \leq 0.25$ from the mean 
infrared relation. To fit this relation we used a robust method based in a Huber M-estimate (Birkes and Dodge 1993), that minimizes the influence of outliers. This value of the slope is in good agreement with $m_{V-I}=-0.042 \pm 0.004(z=0.33)$ and $m_{V-I}=-0.067 \pm 0.007(z=0.55)$, obtained from HST observations (Gladders et al. 1998). Once the cluster galaxy colours have been corrected by the adopted slope, we compute the mean colour and dispersion using a biweight algorithm (Beers et al. 1990), that minimizes the presence of outliers. We additionally, compute the mean colour and dispersion using a simple average, median and average, and median using a $3 \sigma$ clipping to estimate the effect of outliers in the final results. All the errors in the parameters were determined using a bootstrap technique, and they represent the $68 \%$ confidence interval.

To compare the mean colour of the galaxies in each of the clusters it is necessary to adopt a reference magnitude for which we measure its colour. Here, we decided to adopt an empirical approach again, and use a fixed apparent magnitude of $I=19.4$ corresponding to an $\mathrm{M}^{*}$ galaxy at $z=0.45$, as determined in Chapter 4 . In using a fixed apparent magnitude to measure the mean colour for each of the clusters, we are to first order neglecting the effect of having a non-zero slope in the colourmagnitude relation. This approach would produce a small colour bias because we are measuring the colour at a slightly different absolute magnitude due to the small redshift differences from cluster to cluster. Although this effect is small it will be discussed and corrected later in the context of the cluster to cluster dispersion.

The mean galaxy colours and dispersions determined using different samples and photometric methods are presented in Table 5.1. The different methods quoted in the 10th column of this table correspond to the following:

Method 1 Includes all the galaxies within the central $1 \mathrm{Mpc}$ classified as ellipticals and the colours have been determined from the galaxy profile fitting code. All the galaxies selected under this criteria are shown as solid symbols in Figure 5.6 .

Method 2 Uses the galaxies with confirmed redshifts, with no emission lines or 


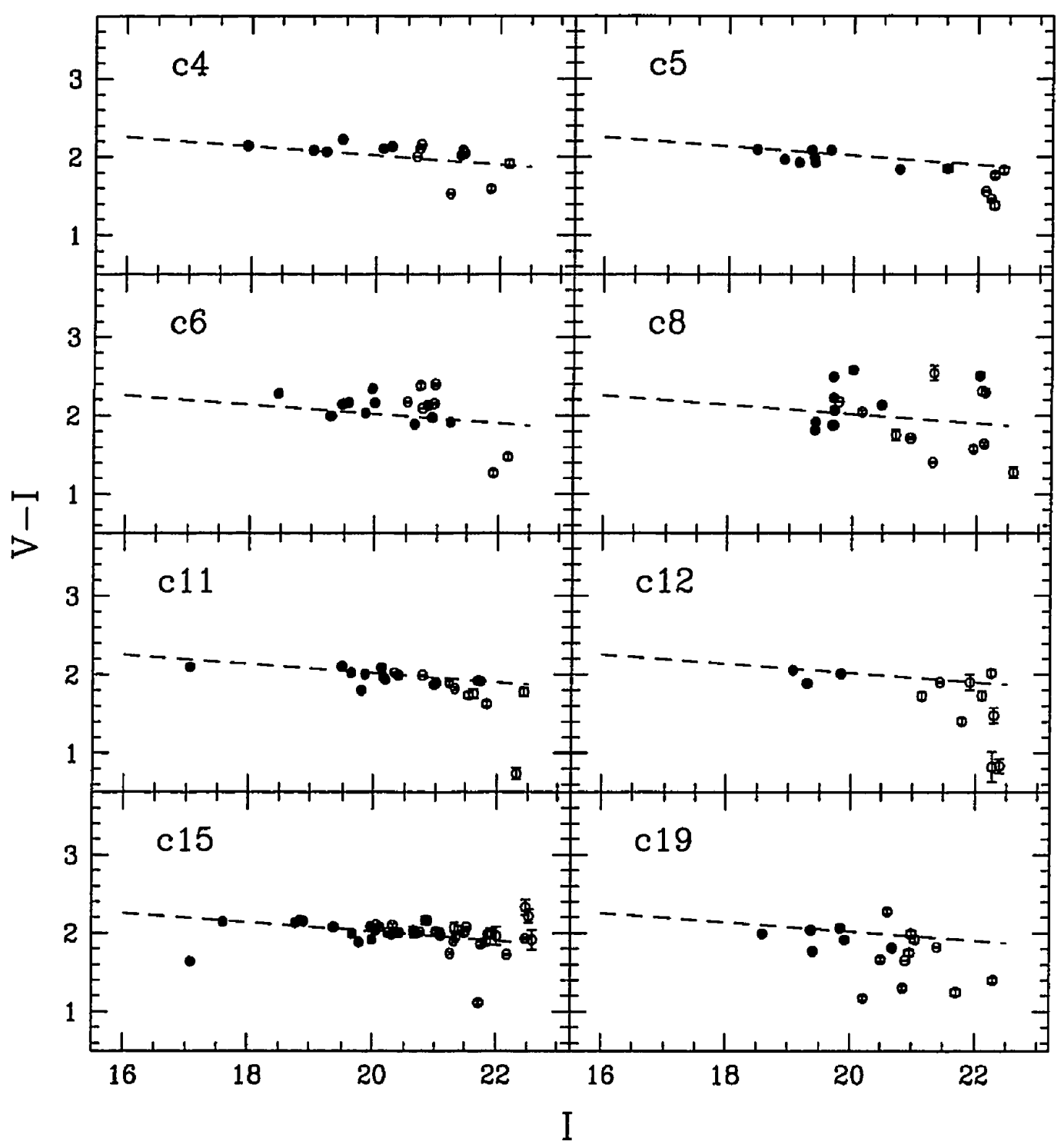

Figure 5.6: Optical colour-magnitude diagram for the elliptical galaxies in the central $1 \mathrm{Mpc}$ of the cluster fields. These galaxies have been also selected for having $\Delta(I-$ $K) \leq 0.25$ from their linear fit. Solid symbols show visually confirmed ellipticals, while open circles denote galaxies classified as undefined, i.e. they could be a bulge or a disk dominated galaxy. The photometric errors in the colours are shown, although they are usually smaller than the symbol size. The dashed line represents the fit for cluster 15. 
Table 5.1: Observed cluster mean colour and colour dispersions.

\begin{tabular}{|c|c|c|c|c|c|c|c|c|c|c|}
\hline Cluster & $(V-I)$ & $\bar{\sigma}_{\bar{V}-I}$ & $\sigma_{V-I}^{+}$ & $\delta$ & $\sigma_{\bar{\delta}}^{\bar{s}}$ & $\sigma_{\delta}^{+}$ & $\operatorname{err}_{V-I}$ & $\bar{N}$ & method & \\
\hline \multirow[t]{6}{*}{$\mathrm{c} 4$} & 1.911 & 0.059 & 0.031 & 0.072 & 0.032 & 0.015 & & 7 & 1 & $\bar{T}$ \\
\hline & 1.893 & 0.034 & 0.034 & 0.054 & 0.042 & 0.007 & & 8 & 2 & \\
\hline & 1.957 & 0.067 & 0.027 & 0.066 & 0.063 & 0.011 & 0.013 & 7 & 3 & \\
\hline & 1.959 & 0.026 & 0.019 & 0.050 & 0.025 & 0.006 & 0.006 & 8 & 4 & $\ddagger$ \\
\hline & 1.927 & 0.029 & 0.018 & 0.069 & 0.024 & 0.038 & & 14 & 5 & \\
\hline & 1.977 & 0.040 & 0.022 & 0.103 & 0.048 & 0.039 & 0.017 & 14 & 6 & \\
\hline \multirow[t]{4}{*}{ c5 } & 2.033 & 0.024 & 0.079 & 0.095 & 0.076 & 0.006 & & 9 & 1 & $\dagger$ \\
\hline & 2.002 & 0.056 & 0.027 & 0.069 & 0.042 & 0.015 & 0.017 & 9 & 3 & $\ddagger$ \\
\hline & 2.033 & 0.024 & 0.079 & 0.095 & 0.076 & 0.006 & & 9 & 5 & \\
\hline & 2.002 & 0.056 & 0.027 & 0.069 & 0.042 & 0.015 & 0.017 & 9 & 6 & \\
\hline \multirow[t]{6}{*}{$c 6$} & 1.955 & 0.050 & 0.045 & 0.129 & 0.033 & 0.022 & & 11 & 1 & $t$ \\
\hline & 1.991 & 0.065 & 0.033 & 0.091 & 0.061 & 0.026 & & 8 & 2 & \\
\hline & 2.014 & 0.046 & 0.050 & 0.122 & 0.034 & 0.023 & 0.015 & 11 & 3 & \\
\hline & 2.055 & 0.041 & 0.024 & 0.079 & 0.031 & 0.026 & 0.007 & 9 & 4 & $\ddagger$ \\
\hline & 2.001 & 0.027 & 0.036 & 0.168 & 0.060 & 0.027 & & 17 & 5 & \\
\hline & 2.065 & 0.032 & 0.043 & 0.170 & 0.054 & 0.029 & 0.019 & 17 & 6 & \\
\hline \multirow[t]{4}{*}{ c11 } & 2.046 & 0.030 & 0.027 & 0.078 & 0.031 & 0.018 & & 9 & 1 & $t$ \\
\hline & 2.014 & 0.037 & 0.012 & 0.079 & 0.038 & 0.028 & 0.012 & 9 & 3 & $\ddagger$ \\
\hline & 2.037 & 0.023 & 0.017 & 0.081 & 0.013 & 0.010 & & 20 & 5 & \\
\hline & 1.994 & 0.032 & 0.018 & 0.091 & 0.021 & 0.018 & 0.022 & 20 & 6 & \\
\hline \multirow[t]{6}{*}{ c12 } & 2.095 & 0.050 & 0.050 & 0.016 & 0.008 & 0.008 & & 3 & 1 & \\
\hline & 2.083 & 0.027 & 0.021 & 0.036 & 0.016 & 0.021 & & 5 & 2 & $\dagger$ \\
\hline & 2.089 & 0.001 & 0.001 & 0.007 & 0.001 & 0.001 & 0.006 & 3 & 3 & \\
\hline & 2.087 & 0.028 & 0.005 & 0.027 & 0.026 & 0.026 & 0.020 & 5 & 4 & $\ddagger$ \\
\hline & 2.097 & 0.142 & 0.003 & 0.057 & 0.048 & 0.092 & & 7 & 5 & \\
\hline & 2.088 & 0.139 & 0.004 & 0.070 & 0.061 & 0.096 & 0.046 & 7 & 6 & \\
\hline \multirow[t]{4}{*}{$\mathrm{c} 15$} & 2.111 & 0.017 & 0.021 & 0.081 & 0.026 & 0.018 & & 17 & 1 & $\dagger$ \\
\hline & 2.079 & 0.016 & 0.017 & 0.069 & 0.031 & 0.015 & 0.019 & 17 & 3 & $\ddagger$ \\
\hline & 2.119 & 0.011 & 0.014 & 0.070 & 0.012 & 0.010 & & 41 & 5 & \\
\hline & 2.097 & 0.014 & 0.008 & 0.070 & 0.012 & 0.014 & 0.028 & 41 & 6 & \\
\hline \multirow[t]{6}{*}{ c19 } & 2.101 & 0.064 & 0.050 & 0.107 & 0.047 & 0.041 & & 6 & 1 & $\dagger$ \\
\hline & 2.116 & 0.026 & 0.057 & 0.051 & 0.050 & 0.011 & & 5 & 2 & \\
\hline & 2.016 & 0.060 & 0.063 & 0.107 & 0.028 & 0.048 & 0.019 & 6 & 3 & \\
\hline & 2.032 & 0.000 & 0.000 & 0.063 & 0.004 & 0.004 & 0.018 & 5 & 4 & $\ddagger$ \\
\hline & 2.052 & 0.078 & 0.079 & 0.304 & 0.132 & 0.063 & & 16 & 5 & \\
\hline & 1.959 & 0.077 & 0.069 & 0.283 & 0.124 & 0.057 & 0.027 & 16 & 6 & \\
\hline
\end{tabular}

$(V-I)$ is the mean observed colour for a $M^{*}(I=19.4)$ galaxy at $z=0.45$ as determined using a biweight technique (Beers et al. 1990). $\delta$ is the observed scatter in the colour when the best mean relation is subtracted. The bootstrap errors in these two parameters are also shown. The total photometric error is denoted by $\operatorname{err}_{V-I}$, while $\mathrm{N}$ is the total number of galaxies used in the fit. The different methods used to compute the mean colour and dispersion are explained in the text. In the last column, $\dagger$ denotes the value chosen as representative of the mean cluster colour, and $\ddagger$ is the chosen value of the observed dispersion. 
confirmed disk dominated morphologies. The colours are from the galaxy profile fitting code for the best bulge fit.

Method 3 The sample is defined as all the morphology confirmed ellipticals within the central $1 \mathrm{Mpc}$. The colours are determined using the 3 arcsec fixed aperture.

Method 4 All the galaxies confirmed as cluster members that do not show emission lines or do not have a dominant disk morphology. The colours are determined from the fixed 3 arcsec aperture.

Method 5 This sample includes all the galaxies confirmed as ellipticals and also all those galaxies having a undefined morphology, i.e. they could be disk or bulge dominated galaxies. The colours are from the galaxy profile fitting code assuming a bulge dominated morphology. All the galaxies under the undefined category are shown as open symbols in Figure 5.6.

Method 6 The sample is selected in the same way as for method 5, but the colours are determined using the 3 arcsec aperture.

As discussed above, the mean galaxy colours for each of the clusters should make use of the colours from the galaxy fitting algorithm, i.e. methods 1,2 or 5 ; while the dispersion should be determined from the fixed 3 arcsec aperture photometry, i.e., methods 3,4 or 6 . As the intrinsic dispersion is proportionally more affected by outliers than the location estimates we decide to adopt the values from method 4 when possible. The values for the mean colour were taken from method I for all the clusters, except for cluster 12 where this method selected only three galaxies. All the adopted values for the mean colour and dispersion are denoted by a $\uparrow$ and $a \ddagger$ in Table 5.1, respectively.

Determining the mean colour value and dispersion for cluster 8 has proven to be an impossible task. The cluster has no additional membership information and its red sequence is not well populated. This cluster corresponds probably to the poorest of the sample when measured by its galaxy content and it is also the faintest in $\mathrm{x}$-rays. 
This cluster will not be included in the analysis, as we have not determined either its mean colour or its colour dispersion.

\subsubsection{Results}

The observed colour dispersion in the $(V-I) / I$ colour-magnitude relation for the clusters in the CTIO sample are $\delta_{o b s}<0.1 \mathrm{mag}$. As the uncertainties in the colours have been carefully determined they can be used to estimate the intrinsic colour dispersion for the galaxies in each cluster. The intrinsic dispersion is then computed from the observed dispersion and the observed photometric errors, in the following way (Akritas and Bershady 1996):

$$
\delta=\sqrt{\delta_{o b s}^{2}-\frac{1}{N} \sum \sigma_{i}^{2}}
$$

where $\delta_{o b s}$ corresponds to the observed dispersion, $\sigma_{i}$ is the photometric error in the $(V-I)$ colour for the $i$-th galaxy, and $N$ is the number of galaxies in the sample.

The intrinsic dispersion for the galaxies in the red sequence for each of the clusters computed in this way are presented in Table 5.2, and also shown in Figure 5.8. All the clusters in this sample are consistent with a small single intrinsic dispersion of $\delta=0.06 \pm 0.02 \mathrm{mag}$, in their observed $(V-I)$ colours. Note that the $(V-I)$ colour at $z=0.45$ corresponds nearly to restframe $(U-V)$.

\section{Observational result \#3}

The intrinsic restframe $(U-V)$ colour dispersion in seven clusters of the CTIO sample at $z=0.45$ is $\delta=0.06 \pm 0.02$.

The observed dispersion values measured here are consistent with those obtained for three clusters at $z=0.54$ (Ellis et al. 1997). The intrinsic dispersion is also consistent with $\delta \simeq 0.06 \pm 0.01$ for the $\mathrm{E} / \mathrm{S} 0$ galaxies in the cluster CL0016+16, from the same work.

The intrinsic dispersion determined here is also consistent with those of Standford et al. (1998) for a sample of clusters at $0.3<z<0.9$, although their uncertainties 
are considerably larger.

\subsection{Dispersion from cluster to cluster}

Before computing the dispersion from cluster to cluster we need to correct the observed mean colours for three different effects. The clusters are at different redshifts, therefore we need to compute accurate $k$-corrections as our photometric system is sampling slightly different spectral regions for the galaxies in these clusters. Also, as mentioned above, the use of a single apparent magnitude to estimate the mean galaxy colour in a cluster introduces a bias that needs to be taken into account. And finally, while most of the clusters are at high galactic latitude having small or null galactic extinction, some of them need to be corrected to make the comparison possible.

\subsubsection{Corrections to the mean colours}

A key point of this work is that the sample of galaxy clusters was chosen to be in a small redshift range in order to minimize the differential $k$-corrections. This has a clear effect here, where we correct the colours and magnitudes to the reference redshift $z=0.45$, i.e. a cluster at $z=0.45$ has no corrections applied. We computed the differential $k$-corrections using the trasmition curves for our filter system and the spectral energy distributions for an elliptical galaxy by Coleman et al. (1980). To estimate their uncertainties we also compute the $k$-corrections including the spectral energy distributions by Bruzual and Charlot (1993) for simple stellar populations of different ages. The differences in the differential corrections for our small redshift range are negligible. The effect of the $k$-correction is shown in the upper panel in Figure 5.7. It is very impressive how the $k$-correction can actually take most of the effect of the colour difference between clusters.

In addition to the $k$-correction, we have to evaluate the effect of determining the mean colour of the galaxies in a cluster by using a fixed apparent magnitude as a reference. This correction depends on two quantities: a) the slope of the colour-magnitude relation, and b) the difference in absolute magnitude in this redshift range. The slope 


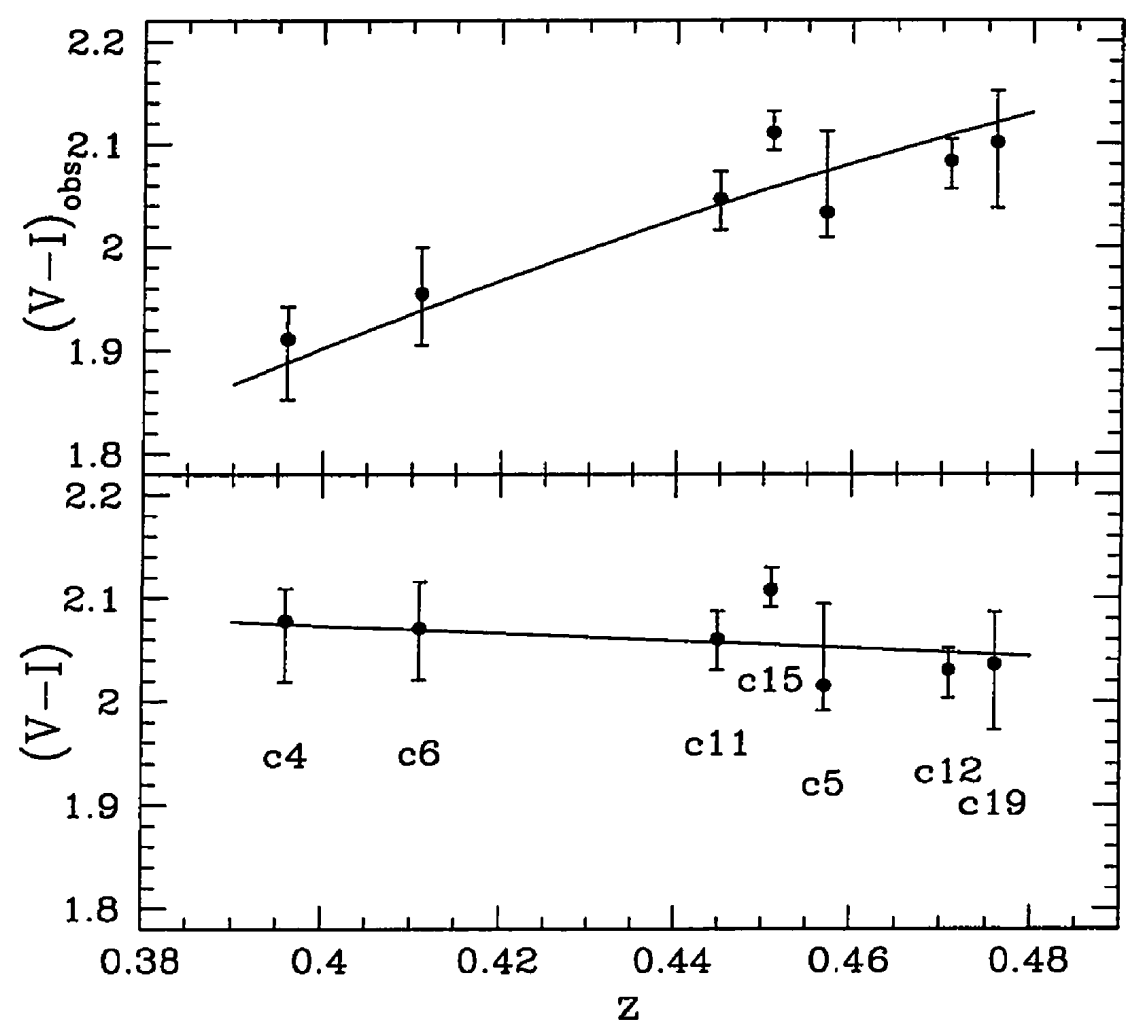

Figure 5.7: Colour corrections. The upper panel shows the observed mean colour for a $I=19.4$ galaxy, for these clusters. The solid line shows the effect of the $k$-correction in the observed colour. The lower panel shows the $k$-corrected mean colours. The residual slope is produced by using a fix apparent magnitude in the determination of the colours. The solid line shows the expected value given the observed slope in the colour-magnitude relation. A galaxy at $z=0.45$ has been defined to have a null correction in both panels.

has been determined empirically from cluster 15, and the magnitude difference for this redshift range depends slightly on the cosmology (there is only $\Delta M=0.03$ mag when changing $q_{0}=0.5$ to $q_{0}=0.1$ ).

When fitting the slope for the relation between the $k$-corrected colours and the differential magnitudes (from the observed redshift to $z=0.45$ ) we obtain $m=$ $-0.091 \pm 0.065$, which is certainly consistent with the value $m=-0.055$ we have been using in the colour and dispersion determinations. These colour corrections, computed using a slope $m=-0.055$, are shown in the lower panel of Figure 5.7. 
Table 5.2: Mean cluster $(V-I)$ colour and intrinsic colour dispersion for the elliptical galaxies for the clusters in the sample.

\begin{tabular}{ccccccc}
\hline \hline Cluster & $(V-I)$ & $\sigma_{V-I}^{-}$ & $\sigma_{V-I}^{+}$ & $\delta$ & $\sigma_{\delta}^{-}$ & $\sigma_{\delta}^{+}$ \\
\hline c4 & 2.058 & 0.059 & 0.031 & 0.050 & 0.025 & 0.006 \\
c5 & 2.018 & 0.024 & 0.079 & 0.067 & 0.042 & 0.015 \\
c6 & 2.057 & 0.050 & 0.045 & 0.079 & 0.031 & 0.026 \\
c11 & 2.058 & 0.030 & 0.027 & 0.078 & 0.038 & 0.028 \\
c12 & 2.038 & 0.027 & 0.021 & 0.018 & 0.026 & 0.026 \\
c15 & 2.108 & 0.017 & 0.021 & 0.066 & 0.031 & 0.015 \\
c19 & 2.046 & 0.064 & 0.050 & 0.060 & 0.004 & 0.004 \\
\hline
\end{tabular}

The $(V-I)$ colour correspond to a $M^{*}$ galaxy $(I=19.4)$ at $z=0.45$. These values are consistent with a single colour of $(V-I)=2.055 \pm$ 0.028 , and a single intrinsic dispersion $\delta=0.060 \pm 0.020$.

The final correction to the colours of the galaxies in these clusters is the galactic extinction. Most of the clusters are located at high galactic latitude and therefore the extinction towards them is low, or null. Clusters $6,11,12$ and 15 have a non-zero value of $A_{B}$ (taken from $\mathrm{NED}^{2}$ ) being $0.06,0.04,0.06$ and $0.13 \mathrm{mag}$, respectively. These values of $A_{B}$ transform into $\mathrm{E}_{(V-I)}$ of $0.024,0.016,0.024$ and 0.052 by using $\mathrm{E}_{(V-I)}=0.4 A_{B}$ (Mihalas and Binney 1981).

Once the extinction, slope and $k$-corrections are applied, we obtain the fully corrected mean colours for the galaxies in each of the clusters, presented in Table 5.2 and plotted in Figure 5.8.

\subsubsection{Estimate of dispersion between clusters}

Figure 5.8 shows that the mean colours for the elliptical galaxies, normalized to an $L^{*}$ galaxy at $z=0.45$, in the clusters of the CTIO sample are consistent with a single mean $(V-I)$ colour. The average of the observed mean $(V-I)$ colours for these clusters corresponds to $(V-I)=2.055 \pm 0.028$. Note that the observed $(V-I)$ colour at $z=0.45$ corresponds approximately to restframe $(U-V)$.

The small uncertainty in the average of the mean cluster colours is completely accounted for by observational uncertainties, and therefore it is consistent with a

\footnotetext{
${ }^{2}$ NASA Extragalactic Database
} 


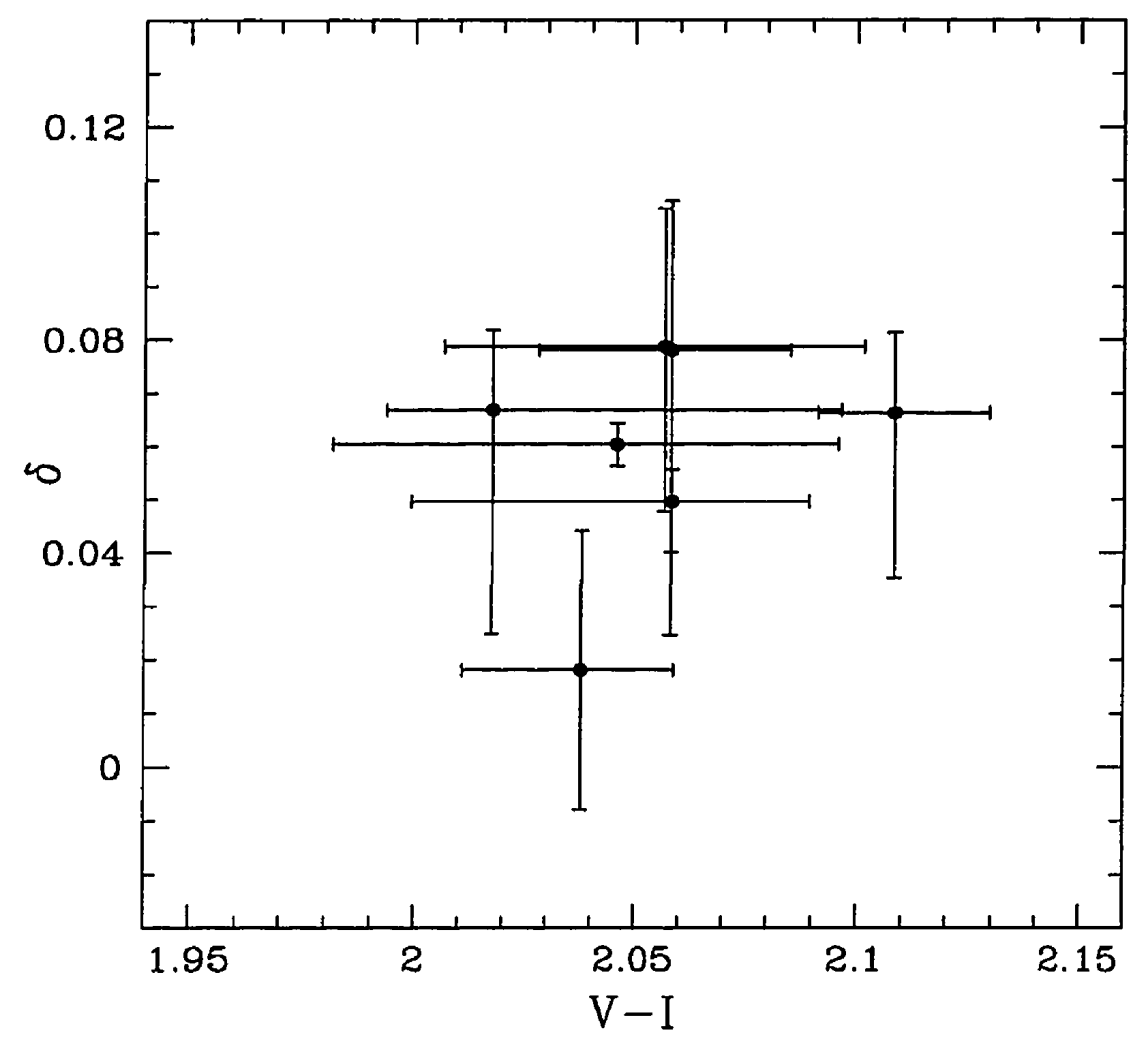

Figure 5.8: Mean $(V-I)$ colour and intrinsic dispersion for the galaxies in each of the clusters of the sample. These colours have been transformed to $z=0.45$. All the clusters are consistent with a single colour $(V-I)=2.055 \pm 0.028$ and with a single intrinsic dispersion $\delta=0.060 \pm 0.020 \mathrm{mag}$.

single mean colour for all the clusters in this sample. Moreover, given the small number of galaxies per cluster to determine the mean cluster colour and the dispersion in the measurement, the small error in the average of the mean cluster colours is accounted for by just sampling errors.

\section{Observational result \#4}

The restframe $(U-V)$ mean colour dispersion between the clusters of the CTIO sample at $z=0.45$ is consistent with zero and lower than $0.028 \mathrm{mag}$.

Ellis et al. (1997) find that the dispersion in the observed mean $(V-I)$ colour for 
three clusters at $z=0.54$ observed with HST is lower than 0.03 mag. This result is consistent with our determination for seven clusters at $z \sim 0.45$.

It is also interesting to note that the richest and most $\mathrm{X}$-ray luminous cluster in the CTIO sample is also the reddest, although the mean colours are consistent with a single value. Unfortunately, this cluster is also the lowest in galactic latitude and has the largest and most uncertain galactic extinction.

\subsection{Discussion - light-weighted ages}

To explore the consequences of the observed small intrinsic dispersions on the evolutionary histories of these galaxies we need to include models of the colour evolution for elliptical galaxies. Figure 5.9 show the expected colour evolution for elliptical galaxies in the $(U-V)$ colour, corresponding nearly to restframe colour for our $(V-I)$ photometry for the galaxies at $z=0.45$. The models shown here are from Bruzual and Charlot (1996), Tantalo et al. (1996) and Worthey (1994), and include the effects of different metallicities. From this figure it is clear that these different models agree fairly well in their overall predictions, while having differences at the level of 0.1-0.2 mag, at most. This figure also shows that the models can be approximated by a linear function in the colour-log $t$ plane, with different slopes for galaxies having different metallicities. This means that we can write,

$$
(U-V)=a \log t+b
$$

By using the linear form and fitting all the models in the time range between 1 and $10 \mathrm{Gyr}$, and comparing the models with approximately the same metallicity we obtain a slope for each metallicity model, including an estimate of its error. We use this error as a measure in the uncertainty introduced by the models in the later analysis. We obtain a value for the slope $a=0.87 \pm 0.07, a=0.70 \pm 0.07$, and $a=0.53 \pm 0.06$, for a high-metallicity $(\mathrm{Z}=0.050)$, solar metallicity $(\mathrm{Z}=0.020)$, and a low-metallicity model $(\mathrm{Z}=0.004)$, respectively. 


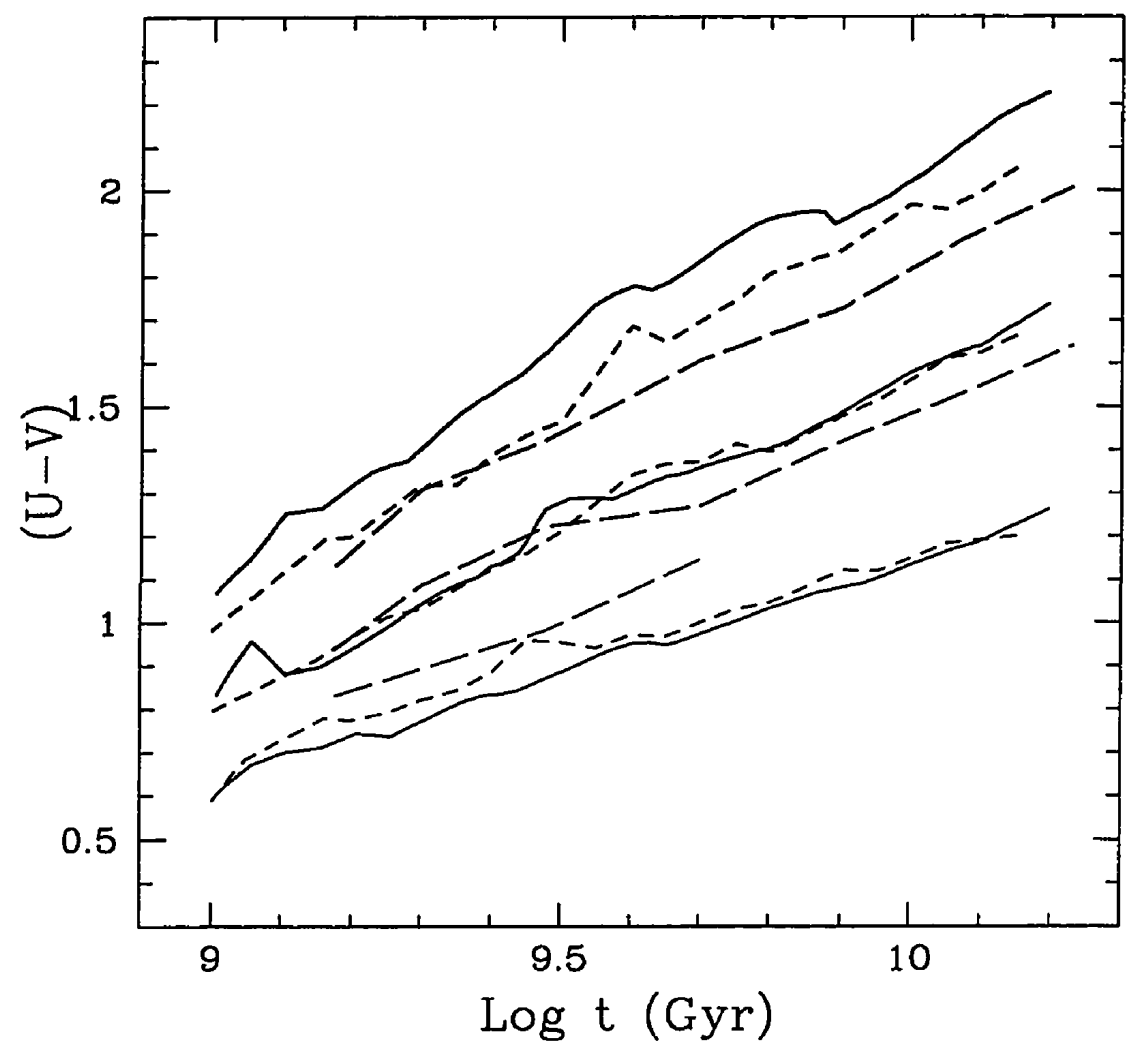

Figure 5.9: Colour evolution of a simple stellar population. The solid line models correspond to Bruzual and Charlot (1996), short-dashed line to Tantalo et al. (1996), and the long-dashed line to Worthey (1994). Light, intermediate and heavy lines corresponding to models with different metallicities, being $\mathrm{Z}=0.004,0.020$ and 0.050 for Bruzual and Charlot (1996) and Tantalo et al. (1996); and $[\mathrm{Fe} / \mathrm{H}]=-0.225,0.000$ and 0.250 for Worthey (1994), respectively.

To see how the dispersion in the $(U-V)$ colour relates to the dispersion in the formation timescale we differentiate the previous expression to obtain

$$
\delta(U-V)=a(\log e) \frac{\delta t}{t}
$$

where $\delta(U-V)$ can be identified with the colour dispersions measured previously, and $(\delta t / t)$ with the time between the single bursts of star formation in two different galaxies, when compared with their mean age.

In this highly idealized scenario the ages of the galaxies correspond unmistakably 
to the ages of the bursts. In real galaxies, stars have been formed over a time period and, since the integrated light we are observing is a mixture of all these stars, we can obtain only a light-weighted age, i.e. the equivalent age of the galaxy when all the stellar population ages have been weighted by their relative contributions to the integrated light.

A slightly more complicated scenario than a single burst is that of assembling the stellar population of the galaxies in two major episodes in the form of single bursts with different masses involved in each of the episodes. We can see the effect of the second burst in timescale formation by modeling the luminosity evolution of each of the bands in the $(U-V)$ colour by an exponential function which is decaying in time, with the difference between their corresponding exponents constrained by the slopes determined from the models in Figure 5.9. If the first burst occurs at $t=0$ and its amplitude is $(1-f)$, and the second burst ocurrs at $t=\Delta t$ (assumed to be $\Delta t<<t$ ) with an ampitude $f$, then the evolution of the mean colour of this galaxy is given by:

$$
(U-V)_{2 b}=a\left(\log t-f \frac{\Delta t}{t}\right)+b
$$

where $\mathrm{b}$ is an arbitrary constant. The light-weighted age of this double burst galaxy corresponds to $t_{2 b}=t(1-f \Delta t / t)$, where $t$ is the age of the first of the two bursts. If the two bursts have the same amplitude, then the correction to the age becomes $\frac{1}{2} \Delta t / t$ which can be seen as a time average between the two bursts ages, weighted by the inverse of the their age.

In this simple model the dispersion in age of the galaxies, the dispersion in the separarion of the two bursts, and the relative strength of the bursts affect the colour dispersion in the following way:

$$
\delta^{2}(U-V)_{2 b}=(a \log e)^{2}\left\{\left(\frac{\delta t}{t}\right)^{2}+\left(f \frac{\delta(\Delta t)}{t}\right)^{2}+\left(\Delta f \frac{\Delta t}{t}\right)^{2}\right\}
$$

As we can see, the second burst has the effect of increasing the observed colour variance produced by the dispersion in time from the initial burst. The variance in 
the colour increases with an increasing variance in the elapsed time between bursts and also increases with the variance in the amplitude of the second burst.

All the treatment above can be generalized for $n$ bursts of the same amplitude, equally spaced in time. The bursts have the same amplitude in order to make the example simpler. In this case the colour is given by

$$
(U-V)_{n b}=a\left(\log t-\frac{(n-1)}{2} \frac{\Delta t}{t}\right)+b
$$

where $\mathrm{b}$ is an arbitrary constant, and $\Delta t$ is the elapsed time between the bursts. The light-weighted age for this galaxy is $t_{n b}=t\left(1-\frac{(n-1)}{2} \Delta t / t\right)$. For this model the colour variance is given by

$$
\delta^{2}(U-V)_{n b}=(a \log e)^{2}\left\{\left(\frac{\delta t}{t}\right)^{2}+\left(\frac{n-1}{2}\right)^{2}\left(\frac{\delta(\Delta t)}{t}\right)^{2}\right\}
$$

And, just as in the previous model, the colour variance is increased by including the $(n-1)$ extra bursts of star formation. The increase in the dispersion is proportional to the numbers of bursts involved and also to the period between the bursts, being always weighted by the inverse of their age.

\subsection{Interpretation}

As shown above, including additional star formation bursts to the galaxies has the effect of reducing the light-weighted age of the galaxies (as expected), and also increases the observed colour dispersion between galaxies relative that of single burst galaxies. Then, by using the single burst galaxy models we obtain an upper limit for the $(\delta t / t)$ ratio. By substituting our measured intrinsic dispersions for the galaxies in each of the clusters, and the slopes from the models we get: $(\delta t / t)=0.16 \pm 0.05$, $(\delta t / t)=0.20 \pm 0.07$, and $(\delta t / t)=0.26 \pm 0.09$ for the high-metallicity, solar metallicity, and low-metallicity galaxies, respectively. Therefore, if the star formation in these galaxies can be approximated by a single burst, then the elliptical galaxies in these 
clusters formed in approximately $20 \%$ of their age at $z=0.45$.

Assuming that these galaxies formed very early in the history of the universe we can estimate the maximum age spread for the formation of these galaxies. If the formation process of these galaxies was not synchronized, then the galaxies have a maximum light weighted-age spread of $10 \pm 3 \%, 13 \pm 4 \%$, and $17 \pm 6 \%$, of the present age of the universe $\left(q_{0}=0.1\right)$, for the high, solar and low metallicity models. These values translates into $\sim 1-2 \mathrm{Gyr}$ for $H_{0} \mathrm{~km} \mathrm{~s}^{-1} \mathrm{Mpc}^{-1}$.

Alternatively, the $(\delta t / t)$ constraint can obviously turn into an age constraint if we know how the age spread in star formation from galaxy to galaxy. If we assume that these galaxies formed under a dissipative gravitational collapse from primeval gas clouds, then the early evolution should be governed by their free fall time, implying that galaxies of $10^{11} M_{\odot}$ should have formed in $\sim 1$ Gyr (Buzzoni 1995). This spread in age translates into an absolute age of 4-6 Gyr, with the higher limit for metal rich galaxies and the lower limit for lower-metallicity galaxies.

Kuntschner and Davies (1998) have determined the metallicities for a sample of galaxies in the Fornax cluster. They find that most of the galaxies there have their metallicities in the range $[\mathrm{Fe} / \mathrm{H}]=0.00,0.50$. This result implies that, if the galaxy formation timescale is $\sim 1 \mathrm{Gyr}$, the bulk of star formation in these galaxies has an age of 5-6 Gyr at $z=0.45$. If the look-back time to $z=0.45$ is about $5 \mathrm{Gyr}$, then these galaxies have a present age of at least 10-11 Gyr.

\subsection{Summary}

In this chapter we have studied the dispersion in the restframe $(U-V)$ colours for the red population of galaxies in the CTIO sample of clusters. We have segregated the galaxies in these clusters by determining their morphology using a 2-dimensional light profile fitting code and supplemented this data with the $(I-K)$ colours. All the clusters, except cluster 8 , have their red sequences clearly defined in the $(V-I) / I$ plane. The dispersions around the colour-magnitude relations for the ellipticals in these clusters are consistent with a single small intrinsic dispersion in restframe $(U-$ 
$V)$ of $\delta=0.06 \pm 0.02$. When comparing the mean colour for the galaxies in each of the clusters, this is consistent with a single value for which the dispersion is smaller than $0.028 \mathrm{mag}$, and completely accounted for by sampling errors.

With the help of spectral synthesis models and by assuming that the stars in these galaxies formed all in a single burst, the intrinsic dispersion in the colours of the galaxies within each cluster is consistent with the galaxies having formed in a fractional timescale $(\delta t / t)=0.16 \pm 0.05,(\delta t / t)=0.20 \pm 0.07$, and $(\delta t / t)=0.26 \pm 0.09$ for a high-metallicity, solar metallicity, and low-metallicity galaxy model, respectively. This fractional timescale can be decreased by using models that include multiple bursts of star formation. 


\section{Chapter 6}

\section{Colour Evolution}

Elliptical galaxies, if they are passively evolving objects, constitute the natural stepping-stone into galaxy evolution from the well-studied globular clusters in the Galaxy. These galaxies, therefore, define a simple and unique laboratory to study the evolution of old stellar populations and thus they can be used to set constraints for the current stellar population synthesis models.

In this chapter we assume that the red galaxy population observed in the clusters of our CTIO sample at $z \sim 0.45$ corresponds to the same population of galaxies seen in local clusters today. This assumption allows us to observe a well defined subsample of the general galaxy population at two widely separated cosmic epochs, and thus to measure the changes in their stellar populations.

\subsection{Galaxy colours at $z=0.45$}

As we have seen in Chapter 5, the elliptical galaxies in the clusters of the CTIO sample form a very homogeneous population in their optical and infrared colours. These galaxies follow a well defined sequence, with very small intrinsic scatter, in the colour-magnitude plane. This sequence has a small negative slope, brighter galaxies being slightly redder than their fainter counterparts.

Our observed $I, V$ and $K$-band correspond nearly to restframe $U, V$ and $H$-band for the galaxies in the clusters at $z=0.45$. This photometry, together with local data, 
allows us to construct the evolutionary vector, in the $(U-V) /(V-H)$ plane, that elliptical galaxies follow from the epoch at $z=0.45$ to the present. Correcting for the slope in the colour-magnitude relation and normalizing to an $\mathrm{L}^{*}$ galaxy $(I=19.4$ for the galaxies at $z=0.45$ as determined from Chapter 4), we obtain the observed mean colours $(V-I)=2.055 \pm 0.028$, and $(I-K)=2.874 \pm 0.016$. In order to take these observed colours to restframe $(U-V)$ and $(V-H)$ we need to apply several small corrections, which we describe below.

Our $K$-band photometry has been calibrated using the UKIRT standards (Casali and Hawarden 1992), and the data for the local reference galaxies has been calibrated into the Caltech photometric system (CIT). This difference in the calibration introduces the colour correction $K_{C I T}=K_{U K I R T}-0.018(J-K)$ (Casali and Hawarden 1992). Elliptical galaxies at $z \sim 0.45$ have $(J-K) \sim 1.6$ (Stanford et al. 1995; Standford et al. 1998), making the correction for our observed $K$-band into the CIT system $-0.030 \mathrm{mag}$, and therefore we obtain the observed colour $\left(I-K_{C I T}\right)=2.904 \pm 0.016$.

We transform our observed $(V-I)$ and $(V-K)$ colours to restframe $(U-V)$ and $(V-H)$ in the following way: 1 ) transform these observed colours first into the $\mathrm{AB}$ system (Oke and Gunn 1983), then 2) correct for the small mismatch between the observed $V, I$ and $K$ filters and $U, V$ and $H$ using known spectral energy distributions for ellipticals, and finally 3) transform the photometry back in the $\alpha$ Lyr system for the restframe $U, V$ and $H$. To transform between the $A B$ and $\alpha$ Lyr systems we use: $U_{A B}=U+(0.735 \pm 0.025), V_{A B}=V+(0.006 \pm 0.006), I_{A B}=I+(0.444 \pm$ $0.013), H_{A B}=H+(1.360 \pm 0.020)$, and $K_{A B}=K+(1.860 \pm 0.020)$, where the optical zero points have been taken from Fukugita et al. (1995) and Binney and Merrifield (1998), and the infrared zero points from Wamsteker (1981) and Binney and Merrifield (1998). The errors have been determined from the difference between the values from the two references. We use the filter transmission curves and several different spectra representing elliptical galaxies to determine the corrections, and their associated errors, for the mismatch between bands. These corrections are:

$$
\left(U_{z=0.00}-V_{z=0.45}\right)_{A B}=+(0.400 \pm 0.032)
$$




$$
\begin{aligned}
\left(V_{z=0.00}-I_{z=0.45}\right)_{A B} & =+(0.047 \pm 0.005) \\
\left(H_{z=0.00}-K_{z=0.45}\right)_{A B} & =-(0.104 \pm 0.024)
\end{aligned}
$$

where the under subscript $z=0.45$ denotes the observed filter band at $z=0.45$. The uncertainties are determined by using different spectral energy distributions for elliptical galaxies.

The spectra included in the computation of these small colour corrections, and their corresponding uncertainties, are those for an elliptical galaxy from Coleman et al. (1980), the models for 6 and 15 Gyr-old SSP from Bruzual and Charlot (1996), and a 15 Gyr spectrum from Worthey (1994). The spectrum from Coleman et al. (1980) includes only the optical region, and therefore it could not be included in the correction for the IR bands.

When including all the transformations and corrections, our observed $V, I$ and $K$ values relate to restframe $U, V$ and $K$ as follows:

$$
\begin{aligned}
(U-V)_{z=0.0} & =(V-I)_{z=0.45}-(0.814 \pm 0.044) \\
(V-H)_{z=0.0} & =(I-K)_{z=0.45}+(0.089 \pm 0.041)
\end{aligned}
$$

Before computing the final colours for these galaxies there is one other small correction to apply. When comparing the photometry for bright stars for the same cluster fields for two different observing runs, we found a photometric zero point difference of $0.038 \mathrm{mag}$ for the $I$-band. We corrected this difference by taking the brightest dataset to $0.038 \mathrm{mag}$ fainter, a procedure that introduces the possibility of a systematic error. To account for the uncertainty in the photometric zero point we correct the $I$-band values by $\Delta I=-0.019 \pm 0.019$.

Applying all the transformations and corrections described above, the restframe colours for an $\mathrm{L}^{*}$ galaxy at $z=0.45$ become: $(U-V)=1.260 \pm 0.055$ and $(V-H)=$ $2.974 \pm 0.048$ (CIT system). 


\subsection{Local ellipticals}

The local reference to compare the restframe colours for the galaxies at $z=0.45$ is defined by the E/SO galaxies in the Coma cluster. This cluster is probably the most well-studied cluster of galaxies having large amounts of data already available in the literature. We use as reference the work done by Bower et al. (1992b) to obtain optical and IR colours, and Recillas-Cruz et al. (1990) for an additional source of IR colours.

Bower et al. (1992b) and Bower et al. (1992a) have obtained high precision photometry for the galaxies in Coma. They found an extremely small scatter in the colour-magnitude relation for the galaxies selected morphologically as ellipticals, and the scatter remains small even when they include the S0s. They estimate their systematic errors as 0.02 and $0.03 \mathrm{mag}$ in $(U-V)$ and $(V-K)$, respectively. $\mathrm{By}$ correcting their data for their computed slopes for their $(U-V) / V$ and $(V-K) / V$ relations $\left(m_{(U-V) / V}=-0.0819 \pm 0.008\right.$, and $\left.m_{(V-K) / V}=-0.0743 \pm 0.011\right)$, and reanalyzing it, we find $(U-V)=1.472 \pm 0.010$ and $(V-K)=3.160 \pm 0.015$. These values represent the E/S0 population, and they are normalized to an $\mathrm{L}^{*}$ galaxy corresponding to $V=14$, using the colour $(V-R)=0.80$ and $R^{*}=13.20$ (López-Cruz 1997). Then, we transform their observed $(V-K)$ colour into $(V-H)$ by using $(H-K)=0.22$ (de Propis et al. 1998), which yields to $(V-H)=2.940 \pm 0.018$. Including the systematic errors in their colours, these transform to the final values of $(U-V)=1.472 \pm 0.022$ and $(V-H)=2.940 \pm 0.036$ (assuming an error of $0.01 \mathrm{mag}$ in $H-K)$.

Recillas-Cruz et al. (1990) have also published infrared photometry for the galaxies in the Coma cluster. Reanalyzing the data for the $\mathrm{E} / \mathrm{S} 0$ population and correcting for the slope in the colour-magnitude relation $\left(m_{(V-K) / K}=0.0978\right)$, we find $(V-K)=$ $3.218 \pm 0.029$ for an $\mathrm{L}^{*}$ galaxy corresponding to $K=11.08$ (de Propis et al. 1998). The $K$-band data of this work has been calibrated using the San Pedro Martir photometric system (OAN) (Carrasco et al. 1991), which transforms into the CIT system by $\left(K_{C I T}-K_{O A N}\right)=(0.0021 \pm 0.0052)+(0.0056 \pm 0.0089)(J-K)$. And therefore, 
Table 6.1: Summary of the restframe colours for the E/S0 galaxies in this study.

\begin{tabular}{lccc}
\hline \multicolumn{1}{c}{ Cluster/Source } & $z$ & $(U-V)$ & $(V-H)$ \\
\hline Coma/Bower et al. (1992b) & 0.023 & $1.472(0.022)$ & $2.940(0.036)$ \\
Coma/Recillas-Cruz et al. (1990) & 0.023 & & $2.989(0.034)$ \\
Coma/mean & 0.023 & $1.472(0.022)$ & $2.965(0.025)$ \\
CTIO sample/this work & 0.450 & $1.260(0.055)$ & $2.974(0.048)$ \\
difference & & $-0.212(0.060)$ & $0.009(0.054)$ \\
\hline
\end{tabular}

Mean galaxy colours for an $\mathrm{L}^{*}$ galaxy in Coma and the CTIO clusters in this study. The mean colours have been computed using a biweight algorithm and their errors using a bootstrap technique.

by using $(H-K)=0.22$, we obtain $(V-H)=2.989 \pm 0.034$ in the CIT system. This value is in agreement with that determined from using the data in Bower et al. (1992b).

The restframe $(U-V)$ and $(V-H)$ colours for the elliptical galaxies in the clusters of the CTIO sample and Coma are summarized in Table 6.1 and they are shown in Figure 6.1. We have used the $(U-V)$ value from Bower et al. (1992b) to plot in conjunction with the $(V-H)$ from Recillas-Cruz et al. (1990). Note that the apparent mistmatch between the observed colours and those predicted by the models could be minimized if we use a galaxy brighter than $L^{*}$ to determine the colours. Given the colour-magnitude relation for the galaxies in Coma, the colours of a galaxy 2 magnitudes brighter than $L^{*}$ would approximate the colours of a 14 Gyr-old galaxy.

\subsection{Observed colour evolution}

Assuming the galaxies observed in the clusters at $z=0.45$ evolved into the galaxies seen in Coma, implies that their broadband colours have changed, and therefore their stellar populations have also changed. This change manifests itself mainly in the blue region of the spectra as $\Delta(U-V)=-0.212 \pm 0.060$, while the visual-infrared colour is consistent with no change at all, $\Delta(V-H)=0.009 \pm 0.054$ (using the mean of both local determinations as a reference). 


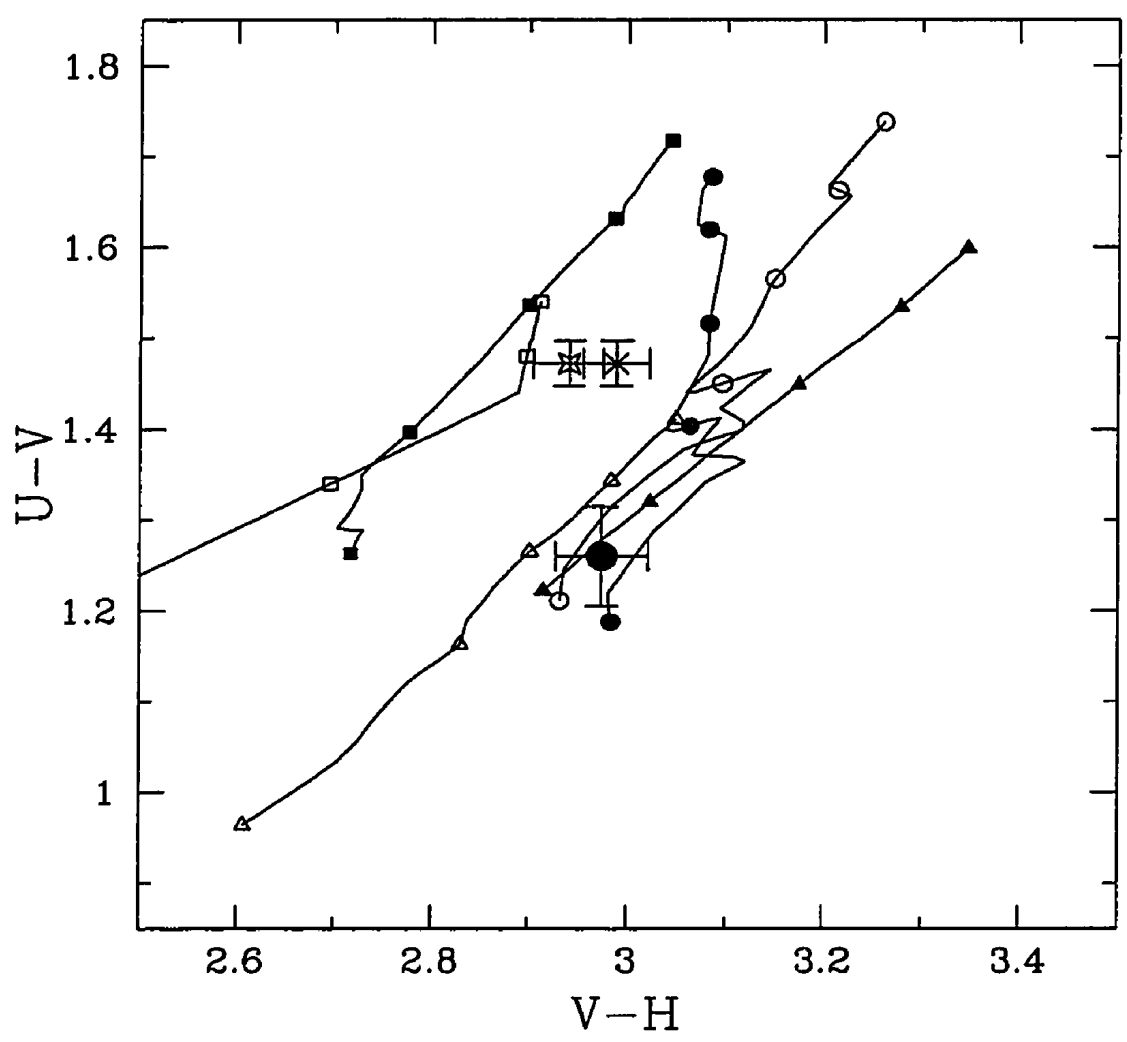

Figure 6.1: $(V-H) /(U-V)$ colour evolution predicted for different SSP models. The models correspond to : open circles for Bressan et al. (1994), solid circles for Tantalo et al. (1996), open triangles for Fioc and Rocca-Volmerange (1997), solid triangles for Worthey (1994), open squares for Arimoto and Yoshii (1987), and solid squares for Bruzual and Charlot (1996). The symbols are located at the ages: 3, 6, 9, 12 and 15 Gyr from the initial burst of star formation. The reddest symbols denotes the colour of the galaxy at $\tau=15$ Gyr. The data points correspond to: star for Bower et al. (1992a), cross for Recillas-Cruz et al. (1990), and filled large circle for this work. The data point for Recillas-Cruz et al. (1990) did not have a $(U-V)$ value, so we adopted the value from Bower et al. (1992a). 
Table 6.2: Colour evolution of elliptical galaxies from the literature and this work.

\begin{tabular}{lclll}
\hline \multicolumn{1}{c}{ Source } & $z$ & colour & change & comment \\
\hline Couch and Shanks (1985) & 0.57 & $\Delta(V-I)$ & $=-0.40 \pm 0.14$ & observed \\
Lilly (1987) & 0.41 & $\Delta(U-V)$ & $=-0.12 \pm 0.11$ & restframe \\
Aragón-Salamanca et al. (1993) & 0.56 & $\Delta(V-I)$ & $=-0.29 \pm 0.06$ & observed \\
Oke et al. (1996) & 0.50 & $\Delta(g-i)$ & $=-0.18$ & observed \\
Ellis et al. (1997) & 0.54 & $\Delta(V-I)$ & $=-0.37 \pm 0.06$ & observed \\
this work & 0.45 & $\Delta(U-V)$ & $=-0.21 \pm 0.06$ & restframe \\
Lilly (1987) & 0.41 & $\Delta(V-H)$ & $=+0.08 \pm 0.13$ & restframe \\
Aragón-Salamanca et al. (1993) & 0.56 & $\Delta(I-K)$ & $=-0.09 \pm 0.03$ & observed \\
Stanford et al. (1995) & 0.40 & $\Delta(I-K)$ & $=-0.16 \pm 0.11$ & observed \\
this work & 0.45 & $\Delta(V-H)$ & $=+0.01 \pm 0.05$ & restframe \\
\hline
\end{tabular}

Different independent determinations of colour evolution of the early-type galaxies in clusters. This table shows the amount of evolution detected at the redshift of the study.

\section{Observational result \#5}

$$
\begin{aligned}
& \text { The evolution colour vector between } z=0.45 \text { and } z=0 \text { is } \\
& \Delta(U-V)=-0.212 \pm 0.060, \text { and } \Delta(V-H)=0.009 \pm 0.054
\end{aligned}
$$

This observed colour evolution is consistent with previous determinations, particularly in the optical $(U-V)$ colour where there are several independent measurements. The change in the $(V-H)$ colour is certainly smaller than the change observed in $(U-V)$. Although different determinations for the evolution in the $(V-H)$ colour roughly agree, there is a large dispersion among the different determinations. A summary of the colour evolution detections are presented in Table 6.2.

\subsection{Spectral synthesis population models}

The observed colour evolution for the elliptical galaxies in clusters can be used to constrain current spectral synthesis models that predict the integrated colours of their stellar populations as function of its age.

Spectral synthesis models use most of our knowledge of stellar evolution by including: 1) stellar evolution tracks of one or more metallicities, 2) spectral libraries 
that could be empirical or from theoretical model atmospheres, and 3) some set of rules to transform the theoretical data into observables. These models make also use of adjustables parameters such as the initial mass function (IMF), the star formation rate (SFR) and some chemical enrichment law. In some of the models, this later parameter is kept fixed during the evolution of the stellar population, or it could be varied according to dynamical arguments or to some predefined prescription. The output of the models are some observables that can be tested through observations. These observables include typically: 1) galaxy spectral energy distributions, 2) galaxy colours and magnitude as a function of time, and 3) line strength and other spectral indexes as function of age.

Some of these spectral synthesis models, for which we are testing our observational results, are briefly described below, and their most important input parameters are summarized in Table 6.3.

Arimoto and Yoshii (1987), AY87: This model includes galactic chemical evolution for a timescale that is governed by the mass of the galaxy. Chemical enrichment ceases when the gas is heated by supernova explosions and then driven out of the galaxy. The authors interpret the colour-magnitude relation as a metallicity effect, and the dispersion around this relation as the intrinsic dispersion in the star formation rate and/or contamination by young stars.

Bressan et al. (1994), BCF94: This is a chemo-spectrophotometric model for elliptical galaxies which includes an initial period of activity followed by quiescence after the onset of the galactic winds, whose duration depends on the galactic mass. This model is in the same line of that of AY87. They adopt a closed-box description for the chemical evolution.

Bruzual and Charlot (1996), BC96: This is probably the most popular of the models. It is based on synthesizing galaxy spectra by combining single star formation bursts according to a given prescription for its star formation history. In the present work we use the single burst models as representative of the 
Table 6.3: Summary of input parameters for the evolutionary models in this study.

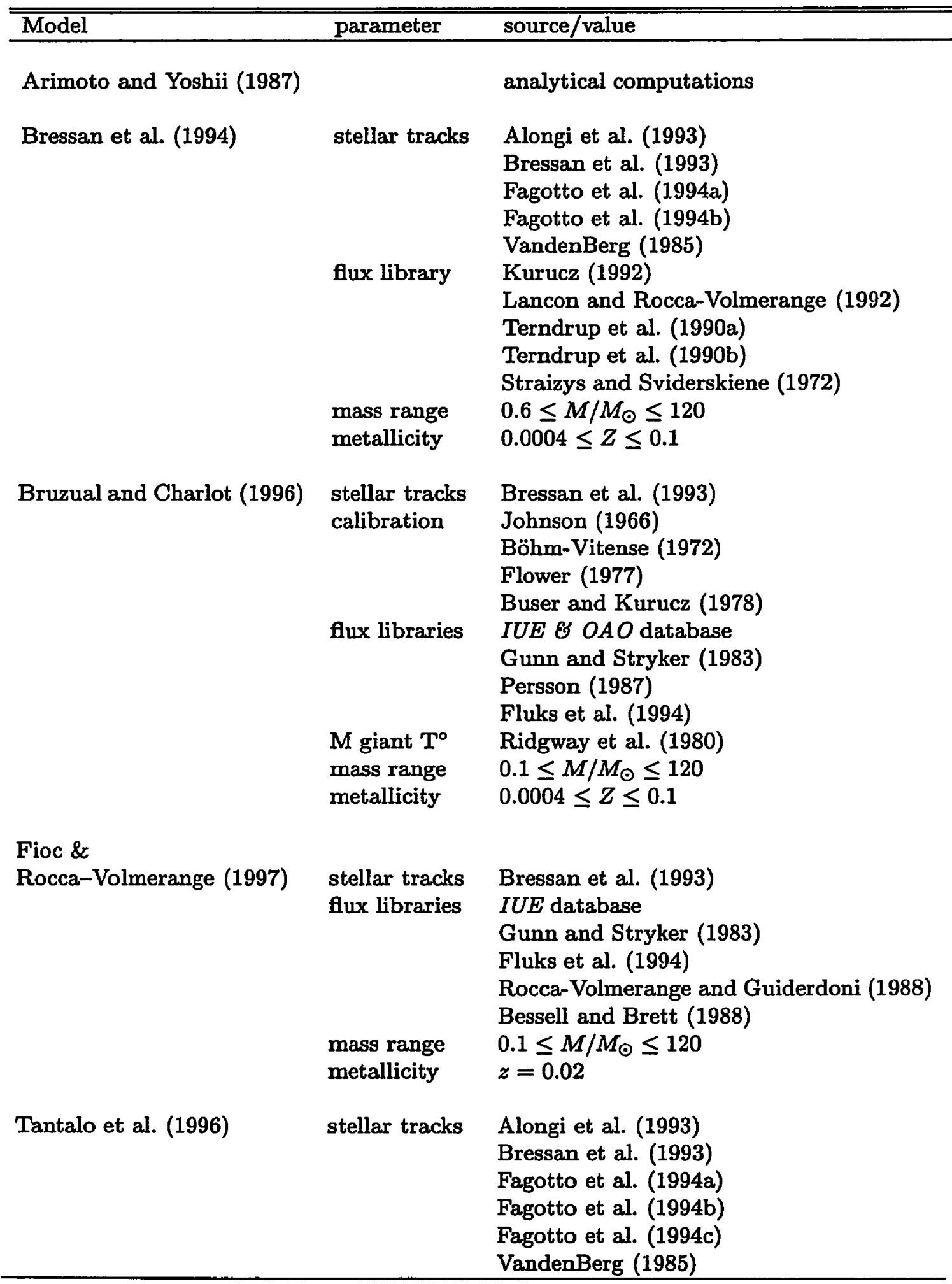


Table 6.3: Continued

\begin{tabular}{lll}
\hline \hline Model & parameter & source/value \\
\hline flux library & Kurucz (1992) \\
& Fluks et al. (1994) \\
mass range & $0.15 \leq M / M_{\odot} \leq 120$ \\
metallicity & $0.0004 \leq Z \leq 0.1$ \\
stellar tracks & VandenBerg (1985) \\
& VandenBerg and Bell (1985) \\
& VandenBerg and Laskarides (1987) \\
& Green et al. (1987) \\
& flux library & Kurucz (1992) \\
& Gunn and Stryker (1983) \\
& Bessell et al. (1989) \\
& Ridgway et al. (1980) \\
& giant T $\mathrm{T}^{\circ}$ & Ridgwey \\
& mass range & $0.1 \leq M / M_{\odot} \leq 2$ \\
metallicity & $0.0001 \leq Z \leq 0.1$ \\
\hline
\end{tabular}

Different input parameters (and their sources) for the models included in the present work.

stellar population of elliptical galaxies. This model does not include chemical evolution but does include a range of metallicities.

Fioc and Rocca-Volmerange (1997), FR97: This model is an updated version of that of Guiderdoni and Rocca-Volmerange (1987). It uses a similar approach of that of $\mathrm{BC} 96$ in synthesizing the colours of ellipticals by using a recipe for their star formation. One of the main features of this model is that it has been extended into the NIR.

Tantalo et al. (1996), TCF96: This is a modified version of the model by BCF94 that includes infall of primordial gas while the stellar population is being assembled. This is aimed to simulate the collapse of a galaxy which is made of two components, luminous and dark matter. This model is constrained to match the properties of local galaxies such as: the colour-magnitude relation, the $U V$ excess, the mass-to-light ratio, broad band colours, and some spectral indexes. This model includes the interpretation of the colour-magnitude relation as a metallicity effect.

Worthey (1994), W94: This model follows the line of that of BC96 where elliptical galaxies are the product of a single burst of star formation. This model 


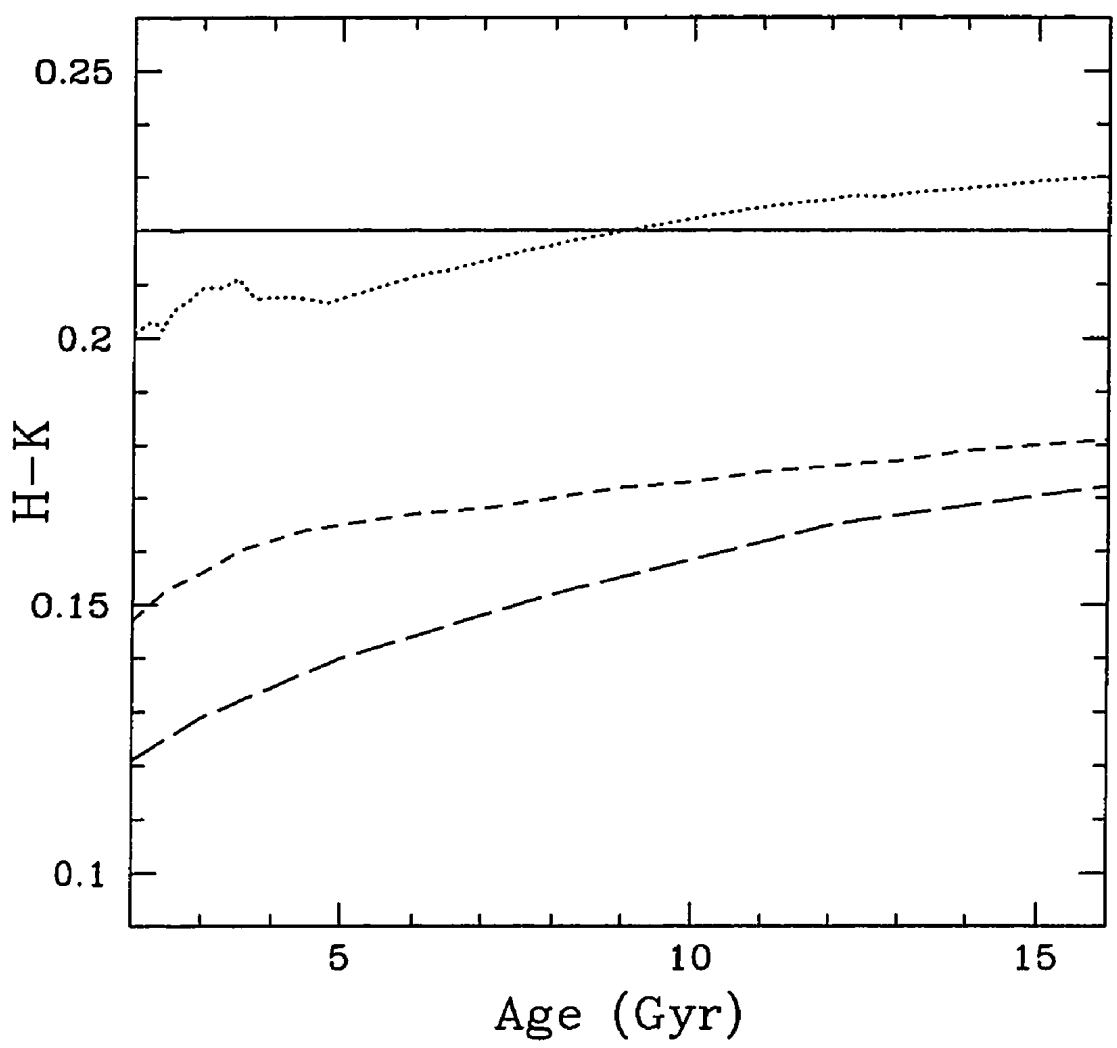

Figure 6.2: $(H-K)$ colour evolution of a simple stellar system predicted by different models: dotted line for Bruzual and Charlot (1996), short-dash line for Fioc and Rocca-Volmerange (1997), long-dash for Worthey (1994). The solid line shows the observed value for elliptical galaxies in Coma (de Propis et al. 1998). This colour remains nearly constant during the lifetime of the galaxy. The maximum predicted change amounts to approximately 0.04 mags for the model by Worthey (1994).

make clear predictions of many spectral features in the spectra, but it is not particularly suited for the IR.

The models by AY87, BCF94 and TCF96, described above, do not include the IR photometric $H$-band in their tables. To transform their published $(V-K)$ values into $(V-H)$ we correct them by using $(H-K)=0.22$, taken from the galaxies in Coma (de Propis et al. 1998). The assumption of a non-evolving ( $H-K$ ) is not a major problem, as it is shown in Figure 6.2, where the prediction of the models show a maximum colour change of $0.04 \mathrm{mag}$ in the period between 3 and $15 \mathrm{Gyr}$. 
Table 6.4: Elapsed time between $z=0.45$ and $z=0.00$ and age of the universe for different cosmological models.

\begin{tabular}{ccccccc}
\hline \hline & \multicolumn{2}{c}{$q_{0}=0.0$} & \multicolumn{2}{c}{$q_{0}=0.1$} & \multicolumn{2}{c}{$q_{0}=0.5$} \\
\cline { 2 - 7 }$H_{0}$ & $\Delta \tau$ & $\tau_{0}$ & $\Delta \tau$ & $\tau_{0}$ & $\Delta \tau$ & $\tau_{0}$ \\
$\mathrm{~km} \mathrm{~s}^{-1} \mathrm{Mpc}^{-1}$ & $\mathrm{Gyr}$ & $\mathrm{Gyr}$ & $\mathrm{Gyr}$ & $\mathrm{Gyr}$ & $\mathrm{Gyr}$ & $\mathrm{Gyr}$ \\
\hline 50 & 6.1 & 19.6 & 6.0 & 16.6 & 5.6 & 13.1 \\
65 & 4.7 & 15.1 & 4.6 & 12.7 & 4.3 & 10.0 \\
75 & 4.0 & 13.1 & 4.0 & 11.0 & 3.7 & 8.7 \\
100 & 3.0 & 9.8 & 3.0 & 8.3 & 2.8 & 6.5 \\
\hline
\end{tabular}

$\Delta \tau$ is the elapsed time (in Gyr) between $z=0.45$ and $z=0.00$ for a given value of $\mathrm{H}_{0}$ and $q_{0}$.

$\tau_{0}$ is the the age of the universe (in Gyr) for the corresponding cosmology.

When comparing the colours for the galaxies in Coma and in the clusters at $z=0.45$ to the prediction of the models, as shown in Figure 6.1, it is clear that none of the models accounts for the colours in an absolute sense, unless we are willing to consider extremely young galaxies at $z=0.45$. The model by FR97 is probably the one that approximates the colours the best, although is not very satisfactory. This disagreement between the observations and the models is not very difficult to understand when we think that there is real lack of good calibration data, especially in the NIR (Worthey 1994).

More interesting than testing the absolute calibration of the models is to see if they can reproduce the evolutionary vector in the $(U-V) /(V-H)$ plane (assuming that we are observing the population of elliptical galaxies at two widely separated epochs in their lifetime). To compare our observations to the spectral synthesis models we normalize the colours of the models to those at 15 and $12 \mathrm{Gyr}$, and similarly we normalize our colours at $z=0.45$ to the colours for the galaxies in Coma. The evolutionary tracks and the observed colours are shown in Figures 6.3, 6.4, 6.5, where the upper panel corresponds to the normalization at $15 \mathrm{Gyr}$ and the lower panel to the normalization at $12 \mathrm{Gyr}$.

From these figures it is clear that not all of the models reproduce the trend observed in the data. Most of the models predict a similar amount of evolution in both colours, while we have found that the change in the $(V-H)$ colour for these galaxies 

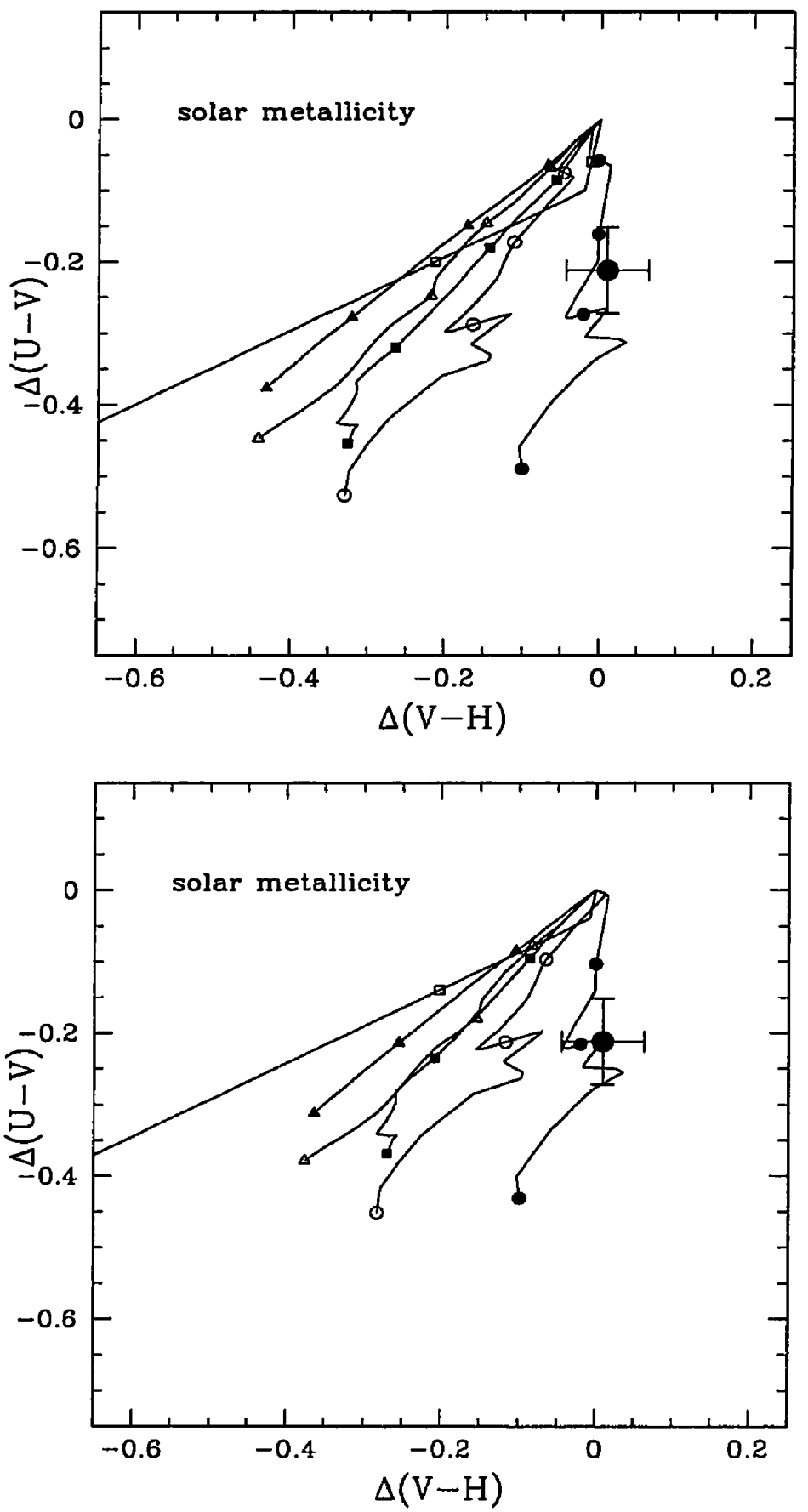

Figure 6.3: Colour evolution normalized to the local determinations. The models are normalized to their values at $15 \mathrm{Gyr}$ (upper panel), and to their values at $12 \mathrm{Gyr}$ (lower panel). The symbols correspond to the same as in Figure 6.1. The model by Arimoto and Yoshii (1987) shown here corresponds to a $10^{11} \mathrm{M}_{\odot}$ galaxy. 

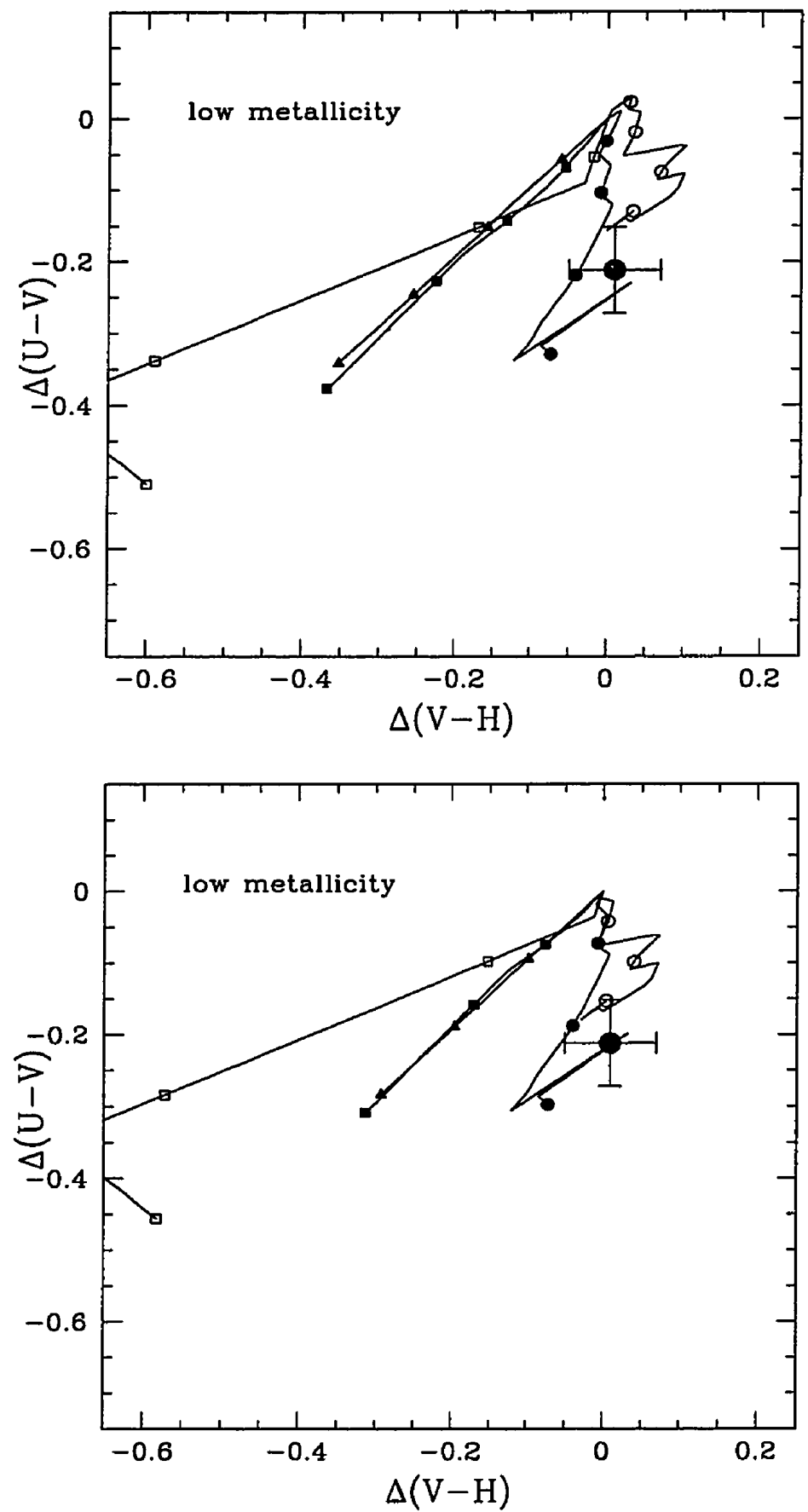

Figure 6.4: Same as in Figure 6.3 for lower metallicity models. The metallicities are: $\mathrm{Z}=0.001$ for Bressan et al. (1994), $\mathrm{Z}=0.004$ for Bruzual and Charlot (1996), $\mathrm{Z}=0.008$ for Tantalo et al. (1996), and $[\mathrm{Fe} / \mathrm{H}]=-0.25$ for Worthey (1994). We also present here the model for a $10^{10} \mathrm{M}_{\odot}$ galaxy for Arimoto and Yoshii (1987). 

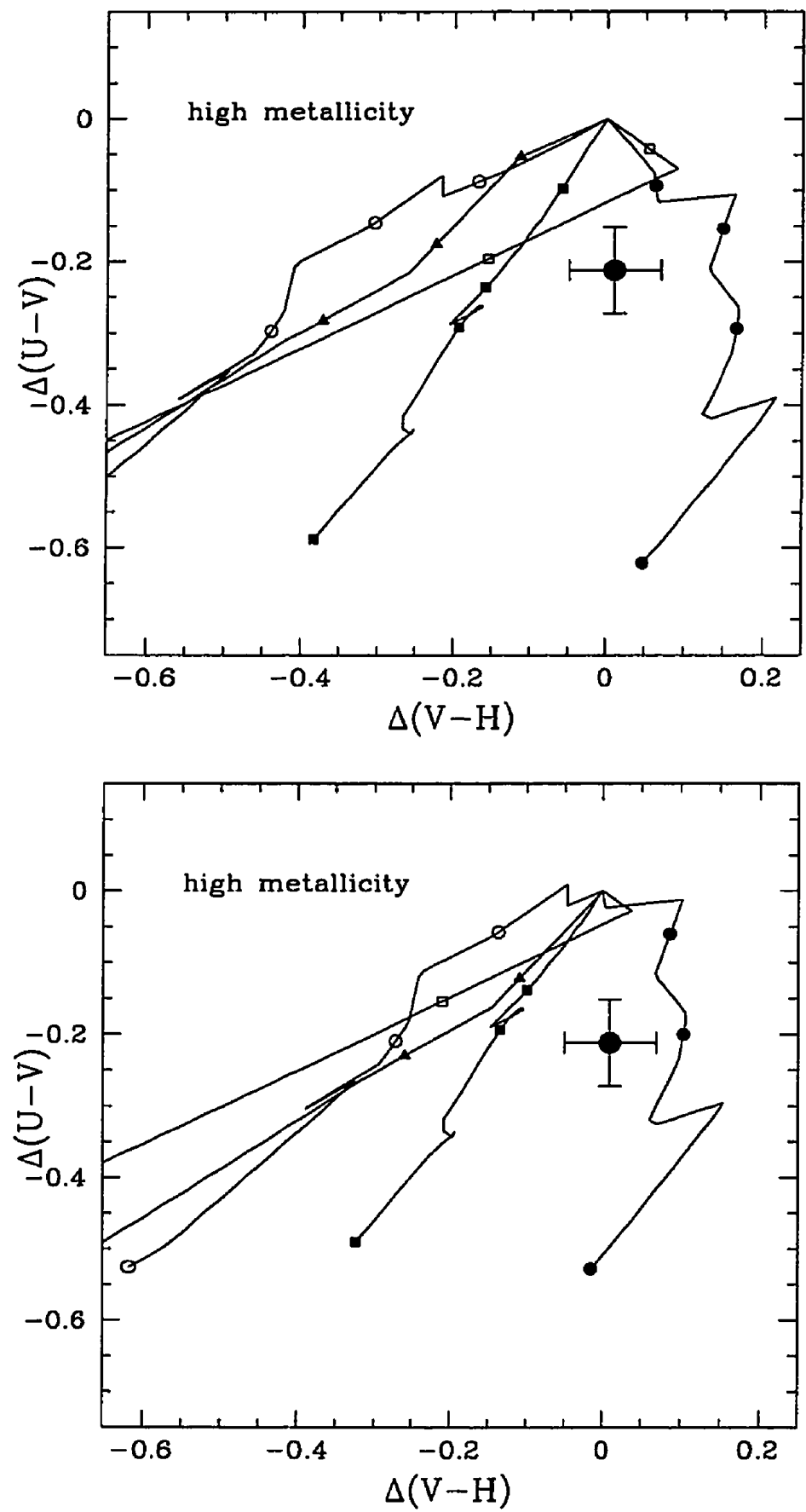

Figure 6.5: Same as in Figure 6.3 for higher metallicity models. The metallicities are: $\mathrm{Z}=0.050$ for Bressan et al. (1994), $\mathrm{Z}=0.050$ for Bruzual and Charlot (1996), $\mathrm{Z}=0.050$ for Tantalo et al. (1996), and $[\mathrm{Fe} / \mathrm{H}]=0.50$ for Worthey (1994). We also include here the model for a $10^{12} \mathrm{M}_{\odot}$ galaxy for Arimoto and Yoshii (1987). 
is consistent with little, or no evolution at all. The TCF96 model with solar or low metallicity reproduce the observed trend very nicely, and it is the only one that is satisfactory. It is interesting to note that this model is an extension of the model by BCF94, sharing most of the stellar libraries and input parameters. Although, the main differences between these two model are: 1) the assumption of initial gas infall in TCF96, while BCF94 use a closed-box approximation, 2) TCF96 includes an improved scale for $T_{\text {eff }}$ as a function of metallicity and new spectra for $M$ stars (Tantalo 1998), and 3) a problem in BCF94, were they gave a wrong temperature to the first point in the HB producing too bluer colours at old ages and high metallicities (Charlot et al. 1996). Unfortunately the impact of this problem is difficult to estimate. The problem in BCF94 can be clearly seen by comparing Figures 6.3, 6.4, and 6.5.

In contrast to the situation for the $(V-H)$ colour, the models agree relatively well in their predictions for the evolution in the $(U-V)$ colour, even for different metallicities, as we saw already in Chapter 5.

The exact nature of the difference between the simple stellar population models included here falls beyond the scope of this thesis. Although, it is clear that the calibrations in the NIR for some of these models are poorly determined leaving room for substantial improvement.

\subsection{Ages for ellipticals}

As we have seen in Chapter 5, and here, the models agree in their predictions for the evolution in the $(U-V)$ colour. By using our observed colour change between $z=0.45$ and $z=0$ and the predicted evolution from the models, we can estimate the light-weighted ages for the stellar populations in these galaxies. We saw that the evolution of the $(U-V)$ colour can be expressed as:

$$
(U-V)=a \log t+b
$$


Table 6.5: Predicted $(U-V)$ colour evolution from the models

\begin{tabular}{cccc}
\hline \hline & $q_{0}=0.0$ & $q_{0}=0.1$ & $q_{0}=0.5$ \\
metallicity & $\Delta(U-V)$ & $\Delta(U-V)$ & $\Delta(U-V)$ \\
\hline 0.050 & $-0.14 \pm 0.01$ & $-0.17 \pm 0.01$ & $-0.21 \pm 0.02$ \\
0.020 & $-0.11 \pm 0.01$ & $-0.14 \pm 0.01$ & $-0.17 \pm 0.02$ \\
0.004 & $-0.09 \pm 0.01$ & $-0.10 \pm 0.01$ & $-0.13 \pm 0.01$ \\
\hline$t_{1} / t_{2}$ & 0.69 & 0.64 & 0.57 \\
\hline
\end{tabular}

The change in the $(U-V)$ colour corresponds to the predicted change between $z=0.45$ and $z=0$, assuming the galaxies formed immediately after the big bang. The ratio $\left(t_{1} / t_{2}\right)$ corresponds to the ratio between the age of the universe at $z=0.45$ and at $z=0$. This colour change compares to $\Delta(U-V)=$ $-0.21 \pm 0.06$ obtained from comparing the observations for the ellipticals at $z=0.45$ with those in Coma.

and therefore, the $(U-V)$ colour change between two different times in the lifetime of these galaxies is:

$$
\Delta(U-V)=a \log \left(t_{1} / t_{2}\right)=a \log \frac{t_{0.45}-t_{f}}{t_{0}-t_{f}}
$$

where $t_{1}$ and $t_{2}$ are the different ages being considered. These ages can be expressed in terms of the age of the universe at $z=0.45\left(t_{0.45}\right), z=0\left(t_{0}\right)$, and the time when these galaxies formed $\left(t_{f}\right)$. Note that in the equation above, the colour change depends only on the cosmology and not on the Hubble constant.

The value of the slope in the equations above depend on the metallicity of the stellar population, being $a=0.87 \pm 0.07, a=0.70 \pm 0.07$, and $a=0.53 \pm 0.06$, for a high metallicity $(Z=0.050)$, solar metallicity $(Z=0.02)$, and low metallicity model $(Z=0.004)$, respectively.

\subsubsection{Consequence of assuming very early formation}

If we assume that these galaxies formed immediately after the big bang, then $t_{f}=0$ and we can compute the color change between $z=0.45$ and $z=0$. These values, for the different metallicity models, are presented in Table 6.5.

With the assumption that the galaxies formed immediately after the big bang, we 
see that low metallicity galaxies in a low density universe have problems to reproduce the observed colour change. On the other hand, solar or high metallicity models can account for it. Although, we have to keep in mind that galaxies must have delay their formation, i.e. $t_{f}>0$, and this has the effect of reducing the ratio $t_{1} / t_{2}$, accommodating a lower density universe better.

\subsubsection{Consequence of adopting low $\Omega$}

If instead of assuming that these galaxies formed immediately after the big bag we use the fact that current studies favor a low value for the matter density in the universe (e.g., $\Omega_{0}=0.24 \pm 0.05 \pm 0.09$, Carlberg et al. 1996) we can estime the epoch, and therefore the age, when these galaxies formed. By using the observed $\Delta(U-V)=-0.21 \pm 0.06$ and the slope values for the different metallicity models, we obtain for the light weighted ages ratio: $\left(t_{1} / t_{2}\right)=0.58 \pm 0.09,\left(t_{1} / t_{2}\right)=0.50 \pm 0.10$, $\left(t_{1} / t_{2}\right)=0.40 \pm 0.11$, for the high, solar and low metallicity models, respectively. Then, if we define:

$$
k=\frac{t_{1}}{t_{2}}=\frac{t_{0.45}-t_{f}}{t_{0}-t_{f}}
$$

we get,

$$
\frac{t_{f}}{t_{0}}=\frac{\left(t_{0.45} / t_{0}\right)-k}{1-k}
$$

where $\left(t_{f} / t_{0}\right)$ corresponds to the fractional time for the formation of these galaxies after the big bang.

By adopting $q_{0}=0.1$, we obtain $\left(t_{f} / t_{0}\right)=0.14 \pm 0.22,\left(t_{f} / t_{0}\right)=0.28 \pm 0.28$, $\left(t_{f} / t_{0}\right)=0.40 \pm 0.39$, for the high, solar and low metallicity models, respectively. By also adopting a relatively low value $\left(\sim 65 \mathrm{~km} \mathrm{~s}^{-1} \mathrm{Mpc}^{-1}\right)$ for the Hubble constant (e.g., Hamuy et al. 1996), we can estimate the ages of these galaxies. These values of the cosmological parameters imply an approximate age of $12.7 \mathrm{Gyr}$ for the universe and about 4.6 Gyr for the elapsed time between $z=0.45$ and $z=0$. Then, the ages of these galaxies are: $10.9 \pm 2.8,9.1 \pm 3.6$, and 7.6 \pm 5.0 Gyr, for high, solar and low metallicity galaxies, respectively. These ages and the cosmology above imply a formation redshift for these galaxies of: $z_{f}=3.7_{-2.4}^{+\infty}, z_{f}=1.8_{-1.2}^{+\infty}$, and $z_{f}=$ 


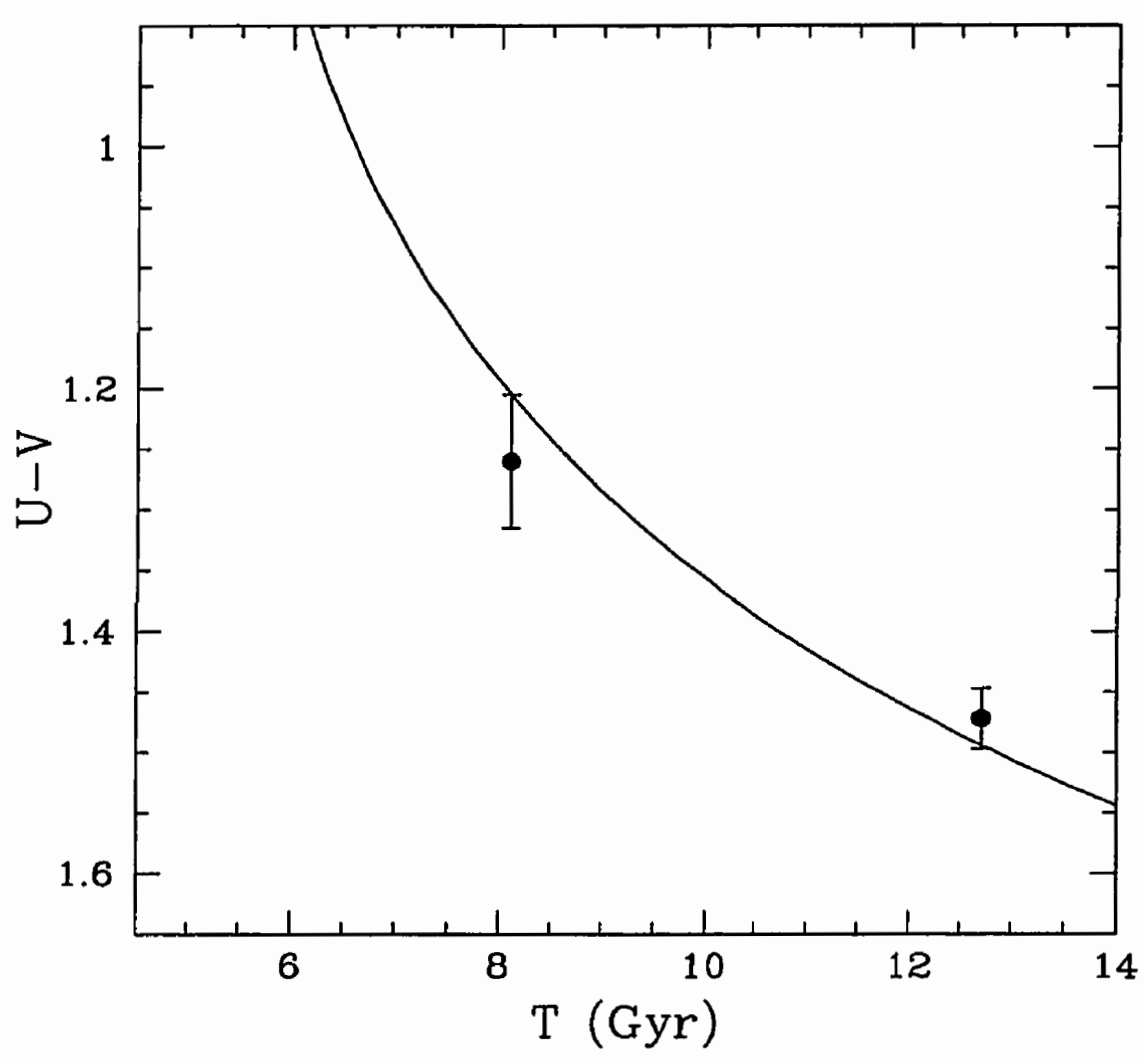

Figure 6.6: Constraint on the ages of these galaxies using the absolute colours. The lowest age for these galaxies is given by the highest metallicity galaxy that can satisfy the observational data and their uncertainties. The lowest present age allowed is 7 Gyr, implying $z_{f}>1$ for a low density universe.

$1.1_{-0.65}^{+\infty}$, respectively. Although, given the local constraints on the metallicities of these galaxies (Kuntschner and Davies 1998), a present age for these galaxies of at least $\sim 6$ Gyr is required.

The ages for these galaxies can also be estimated from the absolute colours and the colour change between the two epochs. Using the colour for only one epoch to estimate the ages of these galaxies would be insufficient because of the well known age-metallicity degeneracy. Worthey (1994) showed that the observed galaxy colours could be equally well matched by two galaxies having different ages and metallicities (see Figure 5.9), constrained to $\Delta \mathrm{age} / \Delta Z \approx 3 / 2$ (i.e. a fractional change of three in the age of a galaxy has the same effect on the colours as a fractional change of two 
in its metallicity). On the other hand, having the colours at two widely separated epochs breaks in part this degeneracy and also sets a joint constraint for the age of the galaxy and its metallicity.

Figure 6.6 shows the youngest galaxy that satisfies the observations, the observed colours and their change between the two epochs. As seen in Figure 5.9, the high metallicity galaxies experience larger changes in the colours than those of lower metallicity. This means that the youngest galaxy satisfying this joint constraint would correspond to that with the highestallowed metallicity. Using the models by BC96, TCF96 and W94 we find that these galaxies must have a present age $t_{\text {gal }}>7 \mathrm{Gyr}$, or $z_{f} \gtrsim 1$, for $q_{0}=0.1$ and $H_{0}=65 \mathrm{~km} \mathrm{~s}^{-1} \mathrm{Mpc}^{-1}$.

\subsection{Summary}

In this chapter we have determined the restframe mean colours $(U-V)=1.260 \pm$ 0.055 and $(V-H)=2.974 \pm 0.048$ for the elliptical galaxies in the clusters at $z=0.45$. If we assume that the galaxies in these clusterss evolve into the same galaxies in Coma, then we observe a colour change $\Delta(U-V)=-0.21 \pm 0.06$ and $\Delta(V-H)=0.01 \pm 0.05$, that is consistent with previous determinations. Most of the spectral synthesis population models of ellipticals included here fail to reproduce this evolutionary vector. Only one of them (Tantalo et al. 1996) predicts small, or no, change in the $(V-H)$ colour. This particular model includes infall of primordial gas and chemical enrichment during the assembly of the stellar population, and also includes improved stellar tracks and calibrations.

By using the spectral synthesis models and the fact the evolution in the $(U-V)$ colour depends on the age of the stellar population we estimate the ages of these galaxies. For a universe having $q_{0}=0.1$ and $H_{0}=65 \mathrm{~km} \mathrm{~s}^{-1} \mathrm{Mpc}^{-1}$, these galaxies are: $10.9 \pm 2.8(Z=0.050)$, and $9.1 \pm 3.6(Z=0.020)$, if we only consider the colour change between $z=0.45$ and $z=0$. On the other hand, if the we also include the absolute colours, then these galaxies should be at least 7 Gyr-old at the present epoch. 


\section{Chapter 7}

\section{Summary and Conclusions}

\subsection{Summary}

In this thesis I present the results from a comprehensive study of the elliptical galaxies in the central regions of galaxy clusters. The observations in this work include deep $V, I$ and $K$-band CCD imaging for eight clusters at $0.39<z<0.48$, taken using the CTIO $4 \mathrm{~m}$, and archival HST imaging for ten clusters of galaxies at $0.2<z<1.2$. All the ground based data has been taken using the same setup, i.e. telescope, filter set, and CCD detector. To obtain high precision photometry for the galaxies in these clusters, we have paid special attention to the observing strategy, reduction, and calibration of the data. We have also taken advantage of already existing software to obtain accurate colours and morphological information.

Through this study we have computed surface brightness for the galaxies, the galaxy luminosity functions, accurate colours and their associated uncertainties. We have studied the colour-magnitude relation for the elliptical galaxies in these clusters and the dispersion in this relation, for the galaxies within clusters and between clusters. This intrinsic colour dispersion contains information about the homogeneity of the elliptical population. We have included published data for local galaxies as reference for our observations, and from the comparison between these two datasets we have tested several popular simple stellar population models. 
Through this thesis we determined five fundamental observational results for the population of elliptical galaxies in clusters. These quantities are listed below:

- The observed luminosity evolution in elliptical galaxies from a surface brightness analysis at $z=1.206$ is $\Delta M_{B}=-1.21 \pm 0.18$.

- The luminosity evolution between $z=0.45$ and $z=0$ from the galaxy luminosity function in clusters is $\Delta M_{V}=-0.57 \pm 0.35\left(q_{0}=0.1\right)$ or $\Delta M_{V}=-0.38 \pm 0.35$ $\left(q_{0}=0.5\right)$.

- The intrinsic restframe $(U-V)$ colour dispersion for the elliptical galaxies in the clusters of the CTIO sample at $z=0.45$ is $\delta=0.06 \pm 0.02 \mathrm{mag}$.

- The restframe $(U-V)$ mean colour dispersion between the clusters of the CTIO sample at $z=0.45$ is consistent with zero and lower than 0.028 mag.

- The evolution colour vector for elliptical galaxies between $z=0.45$ and $z=0$ is $\Delta(U-V)=-0.212 \pm 0.060$, and $\Delta(V-H)=0.009 \pm 0.054$.

These basic observations can be used to constrain the cosmology, models of simple stellar populations and the evolutionary histories of elliptical galaxies in clusters.

Note that all through this thesis work we have defined the elliptical galaxies in the clusters as those objects whose light profile are well represented by an $r^{1 / 4}$-law. This definition generally included the brightest cluster galaxies in the analyzed samples, in spite these galaxies could have a different origin as that of the giant ellipticals we are focusing our study on. The extreme case of these galaxies is that of cluster c15 where there is evidence for a cooling flow (Schindler et al. 1997) and signatures of star formation (Schindler et al. 1995). But, although this galaxy is included in the sample, its impact in the colour dispersion is minimized by the robust statistical techniques applied.

\subsection{Evolutionary history of cluster ellipticals}

In this work we have seen that the morphologically selected elliptical galaxies in 
a sample of galaxy clusters at $z \sim 0.45$ define a very homogeneous colour population. We have seen that the intrinsic dispersion in their restframe $(U-V)$ colours within the clusters is only $\delta=0.06 \pm 0.02$, and this value represents all the clusters in the sample. This small value for the intrinsic dispersion, obtained at a lookback time of about $40 \%$ of the age of the universe, is comparable to that observed in the Coma cluster ( $\delta<0.04 \mathrm{mag}$ for ellipticals only). Additionally, we have also seen that the dispersion in the mean colour from cluster to cluster is lower than 0.028 mag and consistent with zero dispersion.

With the help of simple stellar population models we translate the measured intrinsic dispersion into a timescale constraint for the formation process of these galaxies. We find that the age dispersion over the age ratio for these galaxies at $z \sim 0.45$ is only $(\delta t / t)=0.16 \pm 0.05,(\delta t / t)=0.20 \pm 0.07,(\delta t / t)=0.26 \pm 0.09$ when using a high, solar, and low metallicity model, respectively. Observations of local ellipticals indicate that elliptical galaxies have metallicities between solar and three times solar (Kuntschner and Davies 1998), implying a $(\delta t / t)$ ratio of $\sim 20 \%$ when observed from $z=0.45$. This is quite remarkable because for a universe having a present age of $13 \mathrm{Gyr}$, the $(\delta t / t)$ ratio translates into a maximum age spread of $\sim 1.7$ Gyr. This age dispersion can be reduced by invoking a synchronized galaxy formation process.

The ages of these galaxies can also be estimated by using the observed colour evolution between $z=0.45$ and $z=0$. We know the relative colours of these galaxies at two widely separated epochs and the colour evolution from the simple stellar population models. Then, by adopting a value of $q_{0}$, we determine their relative ages to $z=0.45$ and $z=0$, allowing us to solve for the age of these galaxies. From this approach we obtain the present day ages for these galaxies: $10.9 \pm 2.8(Z=0.050)$ and $9.1 \pm 3.6(Z=0.020)$, adopting $H_{0}=65 \mathrm{~km} \mathrm{~s}^{-1} \mathrm{Mpc}^{-1}$. With the ages computed from the colours and $(\delta t / t)$ ratio, we obtain an age dispersion from galaxy to galaxy of $\sim 1$ Gyr. The ages for these galaxies and our adopted cosmology imply the formation redshifts for these galaxies: $z_{f}=3.7_{-2.4}^{+\infty}(Z=0.050)$ and $z_{f}=1.8_{-1.2}^{+\infty}(Z=0.020)$, and $z_{f} \gtrsim .1$ if we also include the absolute colours in the estimate of the ages. 
We have to note that the ages we are measuring correspond to those of the stellar content in these galaxies (and they really are light-weighted ages) and not necessarily to that of the assembly of the material. In this respect, hierarchical merging models have been able to reproduce the tightness and slope of the colour-magnitude relation, assembling the galaxies at much later times than their stellar content (which is already in place in the smaller units that will make up the galaxy). These models require us to invoke a bias in the selection of the clusters to explain the evolution and the small dispersion in the galaxy colours seen at higher redshifts. Clusters observed at higher redshifts are rare objects that have "turned around" at much earlier epochs than local clusters, and therefore they would mimic the passive evolution observed. To test this bias we would need to study a complete sample of clusters at a given redshift. In our sample, which is not complete but it contains seven clusters at $z=0.45$, we do not see any difference in the mean elliptical galaxy colours for the different clusters, in spite of spanning a wide range in $\mathrm{X}$-ray luminosity. We found that the dispersion in the mean galaxy colours is consistent with zero and smaller than 0.028 mag. Using the same approach to the dispersion within clusters, this observed dispersion translates into $(\delta t / t)<0.1$, that for a low density universe implies a maximum age spread of 1.3 Gyr. This evidence, again, argues in favour of a very synchronized process and/or an early formation of these galaxies. Although the mean colour for the galaxies in different clusters is consistent with no dispersion, there is evidence that the most $\mathrm{X}$-ray luminous and richest cluster in the sample is slightly redder, unfortunately the galactic extinction correction for this cluster is also the most uncertain and it could account for the observed difference.

\subsection{Bottom line}

The results of this thesis have shown that the elliptical galaxies in the central regions of clusters at $z=0.45$ form a remarkably homogeneous population as defined by their stellar populations. Furthermore, the observed colour and luminosity evolution from $z=0.45$ to $z=0$ is consistent with that expected from an old population, 
in standard cosmologies, evolving passively (i.e. with no continuing star formation).

These observations strongly suggest that these galaxies are old systems formed at high redshift. However, the observations do not rule out scenarios involving formation at lower redshifts $(z \sim 1)$, given the quantitative uncertainties in the observations, the spectral population models, the cosmology, and uncertainties in how much synchronicity in formation is reasonable. In this sense, the actual quantitative analysis in this work, which is based on data as good as any up to now, does not support the claims in the literature that these galaxies must be old systems that must have been formed at $z \geq 3$.

\subsection{Future work}

Star formation in galaxies that reside in clusters is advanced with respect to the field, and therefore, testing the predictions of hierarchical structure formation using cluster ellipticals requires us to observe these galaxies at relatively higher redshifts. In a cluster having a velocity dispersion of $1000 \mathrm{~km} \mathrm{~s}^{-1}$, half of the stars are already in place by $z \sim 2$, while for the corresponding field galaxies this happen at $z \sim 1$ (Baugh et al. 1998). The current searches for high redshift clusters together with the $8 \mathrm{~m}$-class telescope and their improved infrared arrays will allow to extent this work into higher redshifts.

An alternative approach is to use the field ellipticals to constrain these models. This method requires a deep field survey with large enough area to detect these galaxies. In this respect, I am already part of collaboration using optical and infrared data to search for these objects up to $\sim 3$.

In addition, the data used in this thesis work has not been fully exploited yet. The data was taken under good seeing conditions and therefore it is possible to carry on a weak lensing analysis of these clusters. This would help to characterize the clusters beyond their galaxy contents. In a similar line, when visually inspecting the residuals from the galaxy profile fits it was noted that several of the brighter ellipticals have residuals resembling those multiple images produced by strong lensing. To study the 
nature of these possible lensed galaxies is an interesting follow up project that I would like to pursue. Finally, in the infrared observations of two of these clusters there are two candidates for high redshift clusters that, according to their colours should be at $z \sim 0.8-1$. Verifying spectroscopically the existence of these clusters will be undertaken. 


\section{Appendix A}

\section{Catalogs}

The following tables present the photometric data for the galaxies in the clusters of the CTIO sample analyzed in this work. These catalogs include all the galaxies in the central $1 \mathrm{Mpc}$ of the cluster fields, down to $I=22.5$, and having $\Delta(I-K) \leq$ 0.25 from the relation $(I-K)=-0.055 I+3.951$, obtained from all the cluster fields. These catalogs show the galaxy positions (RA and DEC), the observed $I$ band magnitude computed using sextractor, the $(V-I)_{3}$ colour computed in a 3 arcsec diameter aperture, the $(V-I)_{f i t}$ colour obtained from the light galaxy profile fitting technique, and the morphological classification as explained in Chapter 5. The colours are observed values, and they have not been corrected by galactic extinction.

\section{Photometry and morphology for cluster 4}

\begin{tabular}{cccccccc}
\hline \hline $\mathrm{RA}(2000)$ & $\operatorname{Dec}(2000)$ & $I$ & $\operatorname{err}_{I}$ & $(V-I)_{3}$ & $\operatorname{err}_{(V-I)_{3}}$ & $(V-I)_{\text {fit }}$ & Morph \\
\hline $3: 34: 20.08$ & $-38: 53: 59.7$ & 17.944 & 0.002 & 1.976 & 0.006 & 1.898 & 1 \\
$3: 34: 24.46$ & $-38: 53: 54.4$ & 19.001 & 0.004 & 1.917 & 0.005 & 1.868 & 1 \\
$3: 34: 17.63$ & $-38: 54: 34.5$ & 19.204 & 0.004 & 1.900 & 0.005 & 1.869 & 1 \\
$3: 34: 20.41$ & $-38: 53: 17.9$ & 19.395 & 0.004 & 1.919 & 0.007 & 1.851 & 3 \\
$3: 34: 21.05$ & $-38: 53: 50.2$ & 19.440 & 0.005 & 2.054 & 0.009 & 1.999 & 1 \\
$3: 34: 19.74$ & $-38: 54: 09.9$ & 19.487 & 0.006 & 1.703 & 0.003 & 1.663 & 2 \\
$3: 34: 20.04$ & $-38: 54: 04.8$ & 19.900 & 0.006 & 1.933 & 0.002 & 1.839 & 6
\end{tabular}




\begin{tabular}{|c|c|c|c|c|c|c|c|}
\hline 3:34:21.91 & $-38: 54: 34.3$ & 20.112 & 0.007 & 1.938 & 0.001 & 1.892 & 1 \\
\hline 3:34:23.76 & $-38: 53: 08.2$ & 20.249 & 0.008 & 1.967 & 0.005 & 1.916 & 1 \\
\hline $3: 34: 22.93$ & $-38: 54: 14.1$ & 20.553 & 0.010 & 1.514 & 0.018 & 1.439 & 2 \\
\hline $3: 34: 24.36$ & $-38: 53: 41.4$ & 20.663 & 0.015 & 1.838 & 0.007 & 1.815 & 5 \\
\hline 3:34:21.11 & $-38: 54: 06.5$ & 21.033 & 0.026 & 1.826 & 0.012 & 1.663 & 6 \\
\hline 3:34:23.90 & $-38: 54: 49.2$ & 20.711 & 0.012 & 1.943 & 0.017 & 1.889 & 5 \\
\hline $3: 34: 22.82$ & $-38: 54: 01.4$ & 20.734 & 0.016 & 1.989 & 0.009 & 1.857 & 5 \\
\hline 3:34:17.53 & $-38: 54: 02.6$ & 20.796 & 0.014 & 1.438 & 0.003 & 1.373 & 2 \\
\hline $3: 34: 16.59$ & $-38: 54: 00.1$ & 20.845 & 0.012 & 1.296 & 0.003 & 1.246 & 2 \\
\hline $3: 34: 19.84$ & $-38: 53: 48.7$ & 22.015 & 0.060 & 0.864 & 0.135 & 1.228 & 7 \\
\hline $3: 34: 22.57$ & $-38: 54: 21.9$ & 21.197 & 0.017 & 1.365 & 0.013 & 1.327 & 5 \\
\hline 3:34:18.06 & $-38: 54: 00.0$ & 21.382 & 0.021 & 1.853 & 0.031 & 1.823 & 1 \\
\hline 3:34:23.38 & $-38: 54: 16.0$ & 21.808 & 0.040 & 1.392 & 0.039 & 1.361 & 7 \\
\hline $3: 34: 21.62$ & $-38: 54: 04.5$ & 21.411 & 0.022 & 1.915 & 0.030 & 1.875 & 5 \\
\hline 3:34:18.19 & $-38: 54: 07.0$ & 21.439 & 0.024 & 1.877 & 0.019 & 1.822 & 5 \\
\hline 3:34:19.68 & $-38: 53: 23.0$ & 21.996 & 0.035 & 1.812 & 0.031 & 1.669 & 2 \\
\hline 3:34:17.47 & $-38: 53: 41.1$ & 22.526 & 0.063 & 1.354 & 0.064 & 1.322 & 7 \\
\hline 3:34:16.54 & $-38: 53: 55.7$ & 21.849 & 0.025 & 1.426 & 0.031 & 1.388 & 5 \\
\hline 3:34:17.24 & $-38: 55: 02.3$ & 21.885 & 0.026 & 1.430 & 0.016 & 1.367 & 2 \\
\hline 3:34:20.89 & $-38: 54: 02.3$ & 22.338 & 0.046 & 1.529 & 0.046 & 1.266 & 7 \\
\hline $3: 34: 16.65$ & $-38: 53: 49.2$ & 22.147 & 0.037 & 1.748 & 0.047 & 1.665 & 5 \\
\hline $3: 34: 23.56$ & $-38: 54: 21.0$ & 22.938 & 0.087 & 0.968 & 0.007 & 1.020 & 7 \\
\hline 3:34:18.06 & $-38: 54: 00.0$ & 21.382 & 0.021 & 1.853 & 0.031 & 2.172 & 6 \\
\hline $3: 34: 22.93$ & $-38: 54: 14.1$ & 20.553 & 0.010 & 1.514 & 0.018 & 1.042 & 2 \\
\hline $3: 34: 23.36$ & $-38: 53: 43.5$ & 22.371 & 0.045 & 1.622 & 0.017 & 1.371 & 6 \\
\hline $3: 34: 19.84$ & $-38: 53: 34.4$ & 22.476 & 0.057 & 1.646 & 0.056 & 1.575 & 6 \\
\hline
\end{tabular}




\section{Photometry and morphology for cluster 5}

\begin{tabular}{|c|c|c|c|c|c|c|c|}
\hline $\mathrm{RA}(2000)$ & $\operatorname{Dec}(2000)$ & $I$ & $\operatorname{err}_{I}$ & $(V-I)_{3}$ & $\operatorname{err}_{(V-I)_{3}}$ & $(V-I)_{f i t}$ & Morph \\
\hline 3:39:25.64 & $-35: 29: 16.2$ & 18.454 & 0.003 & 2.112 & 0.008 & 2.166 & 1 \\
\hline 3:39:25.64 & $-35: 29: 37.6$ & 18.889 & 0.004 & 1.988 & 0.003 & 2.047 & 1 \\
\hline 3:39:21.48 & $-35: 29: 21.6$ & 19.134 & 0.006 & 1.948 & 0.012 & 2.014 & 1 \\
\hline 3:39:26.31 & $-35: 28: 38.9$ & 19.228 & 0.005 & 1.512 & 0.006 & 1.580 & 2 \\
\hline $3: 39: 25.32$ & $-35: 28: 38.2$ & 19.363 & 0.004 & 2.009 & 0.006 & 2.117 & 1 \\
\hline 3:39:25.55 & $-35: 29: 02.6$ & 19.329 & 0.007 & 2.101 & 0.006 & 2.183 & 1 \\
\hline 3:39:26.35 & $-35: 29: 03.1$ & 19.379 & 0.007 & 1.946 & 0.002 & 1.986 & 1 \\
\hline $3: 39: 25.85$ & $-35: 29: 42.6$ & 19.641 & 0.007 & 2.107 & 0.011 & 2.219 & 1 \\
\hline $3: 39: 25.69$ & $-35: 29: 10.3$ & 19.720 & 0.007 & 1.877 & 0.004 & 1.945 & 4 \\
\hline $3: 39: 25.54$ & $-35: 28: 53.8$ & 20.112 & 0.010 & 1.434 & 0.012 & 1.333 & 4 \\
\hline $3: 39: 30.12$ & $-35: 28: 57.0$ & 20.246 & 0.010 & 1.585 & 0.005 & 1.659 & 2 \\
\hline $3: 39: 27.09$ & $-35: 29: 42.2$ & 20.756 & 0.014 & 1.861 & 0.011 & 1.932 & 1 \\
\hline $3: 39: 25.33$ & $-35: 29: 34.9$ & 21.086 & 0.017 & 1.845 & 0.016 & 2.025 & 6 \\
\hline $3: 39: 25.10$ & $-35: 28: 20.3$ & 20.953 & 0.017 & 3.230 & 0.024 & 3.137 & 7 \\
\hline $3: 39: 26.73$ & $-35: 29: 32.9$ & 21.151 & 0.018 & 1.602 & 0.007 & 1.680 & 2 \\
\hline $3: 39: 29.89$ & $-35: 29: 06.1$ & 21.164 & 0.021 & 1.257 & 0.010 & 1.376 & 6 \\
\hline $3: 39: 21.54$ & $-35: 29: 17.0$ & 21.522 & 0.026 & 1.873 & 0.044 & 1.895 & 1 \\
\hline $3: 39: 25.08$ & $-35: 28: 10.4$ & 21.722 & 0.029 & 1.299 & 0.015 & 1.366 & 2 \\
\hline $3: 39: 29.16$ & $-35: 28: 42.3$ & 22.130 & 0.034 & 1.580 & 0.012 & 1.660 & 5 \\
\hline $3: 39: 21.87$ & $-35: 29: 53.2$ & 22.217 & 0.043 & 1.482 & 0.016 & 1.486 & 5 \\
\hline $3: 39: 21.48$ & $-35: 29: 21.6$ & 19.134 & 0.006 & 1.948 & 0.012 & 3.181 & 7 \\
\hline $3: 39: 26.50$ & $-35: 28: 15.1$ & 22.270 & 0.048 & 1.403 & 0.044 & 1.510 & 5 \\
\hline $3: 39: 25.79$ & $-35: 30: 10.5$ & 22.273 & 0.038 & 1.785 & 0.027 & 1.853 & 5 \\
\hline 3:39:26.80 & $-35: 29: 45.4$ & 22.413 & 0.044 & 1.852 & 0.032 & 1.982 & 5 \\
\hline
\end{tabular}




\section{Photometry and morphology for cluster 6}

\begin{tabular}{|c|c|c|c|c|c|c|c|}
\hline $\mathrm{RA}(2000)$ & $\operatorname{Dec}(2000)$ & $I$ & $\operatorname{err}_{I}$ & $(V-I)_{3}$ & $\operatorname{err}_{(V-I)_{3}}$ & $(V-I)_{f i t}$ & Morph \\
\hline $4: 48: 02.31$ & $-20: 37: 35.7$ & 17.936 & 0.003 & 2.136 & 0.005 & 2.087 & 3 \\
\hline 4:48:04.05 & $-20: 36: 48.5$ & 17.992 & 0.002 & 1.505 & 0.003 & 1.422 & 2 \\
\hline $4: 47: 57.62$ & $-20: 37: 28.5$ & 18.003 & 0.003 & 2.058 & 0.001 & 1.955 & 6 \\
\hline 4:48:01.97 & $-20: 37: 25.8$ & 18.455 & 0.004 & 2.188 & 0.008 & 2.137 & 1 \\
\hline $4: 48: 02.51$ & $-20: 36: 27.7$ & 18.667 & 0.004 & 1.689 & 0.003 & 1.523 & 6 \\
\hline $4: 47: 58.68$ & $-20: 36: 44.9$ & 19.080 & 0.004 & 1.895 & 0.004 & 1.841 & 2 \\
\hline 4:48:04.35 & $-20: 36: 47.5$ & 19.201 & 0.004 & 1.556 & 0.010 & 1.450 & 6 \\
\hline $4: 48: 03.43$ & $-20: 38: 36.4$ & 19.296 & 0.006 & 1.904 & 0.002 & 1.840 & 1 \\
\hline 4:48:01.16 & $-20: 38: 02.3$ & 19.327 & 0.005 & 2.083 & 0.010 & 2.010 & 2 \\
\hline $4: 47: 58.21$ & $-20: 37: 35.5$ & 19.467 & 0.006 & 2.051 & 0.004 & 2.021 & 1 \\
\hline 4:48:01.03 & $-20: 37: 54.9$ & 19.564 & 0.005 & 2.073 & 0.002 & 2.004 & 1 \\
\hline $4: 47: 57.81$ & $-20: 37: 39.2$ & 19.597 & 0.007 & 2.081 & 0.009 & 1.920 & 6 \\
\hline $4: 48: 01.40$ & $-20: 37: 16.8$ & 19.794 & 0.011 & 1.683 & 0.011 & 1.598 & 6 \\
\hline $4: 48: 01.56$ & $-20: 36: 58.2$ & 19.844 & 0.013 & 1.944 & 0.021 & 1.882 & 1 \\
\hline 4:48:01.33 & $-20: 36: 27.1$ & 19.953 & 0.010 & 2.249 & 0.008 & 2.187 & 1 \\
\hline 4:48:02.48 & $-20: 37: 56.1$ & 20.003 & 0.010 & 2.070 & 0.009 & 1.991 & 1 \\
\hline 4:48:01.81 & $-20: 37: 32.0$ & 20.184 & 0.008 & 2.072 & 0.011 & 2.050 & 6 \\
\hline 4:48:02.63 & $-20: 36: 47.1$ & 20.516 & 0.018 & 1.800 & 0.010 & -0.003 & 7 \\
\hline $4: 48: 04.54$ & $-20: 38: 14.1$ & 20.379 & 0.011 & 1.708 & 0.010 & 0.702 & 7 \\
\hline $4: 47: 58.58$ & $-20: 36: 47.7$ & 20.326 & 0.009 & 1.707 & 0.004 & 1.645 & 2 \\
\hline $4: 47: 57.31$ & $-20: 37: 35.3$ & 20.526 & 0.012 & 2.082 & 0.011 & 2.000 & 5 \\
\hline $4: 47: 58.89$ & $-20: 37: 04.5$ & 20.603 & 0.012 & 2.052 & 0.014 & 1.992 & 2 \\
\hline $4: 48: 01.03$ & $-20: 36: 28.0$ & 20.735 & 0.014 & 2.288 & 0.035 & 2.208 & 5 \\
\hline $4: 48: 02.36$ & $-20: 37: 41.8$ & 20.729 & 0.013 & 1.971 & 0.008 & 1.981 & 6 \\
\hline $4: 47: 58.73$ & $-20: 37: 38.5$ & 20.649 & 0.014 & 1.802 & 0.007 & 1.735 & 1 \\
\hline $4: 48: 02.43$ & $-20: 38: 19.5$ & 20.781 & 0.013 & 2.001 & 0.002 & 1.938 & 5 \\
\hline $4: 47: 59.21$ & $-20: 37: 30.3$ & 20.857 & 0.016 & 2.039 & 0.023 & 1.973 & 1 \\
\hline
\end{tabular}




\begin{tabular}{llllllll}
$4: 47: 57.33$ & $-20: 37: 56.2$ & 20.921 & 0.017 & 1.886 & 0.008 & 1.814 & 1 \\
$4: 48: 00.85$ & $-20: 37: 30.2$ & 20.952 & 0.015 & 2.063 & 0.016 & 1.973 & 5 \\
$4: 48: 03.48$ & $-20: 37: 41.0$ & 20.964 & 0.017 & 2.307 & 0.018 & 2.243 & 5 \\
$4: 48: 04.69$ & $-20: 38: 04.1$ & 21.225 & 0.020 & 1.826 & 0.036 & 1.753 & 1 \\
$4: 48: 01.73$ & $-20: 37: 29.5$ & 21.437 & 0.015 & 1.978 & 0.014 & 1.847 & 7 \\
$4: 48: 04.06$ & $-20: 38: 16.1$ & 21.455 & 0.019 & 1.698 & 0.022 & -1.612 & 7 \\
$4: 48: 00.68$ & $-20: 36: 27.1$ & 21.917 & 0.048 & 1.175 & 0.036 & 1.043 & 5 \\
$4: 47: 59.27$ & $-20: 37: 33.4$ & 21.774 & 0.027 & 1.938 & 0.004 & 1.793 & 6 \\
$4: 48: 05.68$ & $-20: 38: 30.9$ & 22.155 & 0.047 & 1.384 & 0.040 & 1.288 & 5 \\
$4: 47: 58.21$ & $-20: 37: 35.5$ & 19.467 & 0.006 & 2.051 & 0.004 & 1.375 & 7 \\
\hline
\end{tabular}

\section{Photometry and morphology for cluster 8}

\begin{tabular}{cccccccc}
\hline \hline $\operatorname{RA}(2000)$ & $\operatorname{Dec}(2000)$ & $I$ & $\operatorname{err}_{I}$ & $(V-I)_{3}$ & $\operatorname{err}_{(V-I)_{3}}$ & $(V-I)_{f i t}$ & Morph \\
\hline $5: 13: 30.68$ & $-48: 17: 32.5$ & 19.402 & 0.008 & 1.710 & 0.016 & 1.697 & 1 \\
$5: 13: 37.20$ & $-48: 18: 23.7$ & 19.408 & 0.008 & 1.811 & 0.004 & 1.802 & 1 \\
$5: 13: 34.80$ & $-48: 19: 40.9$ & 20.716 & 0.040 & 1.648 & 0.065 & 1.546 & 5 \\
$5: 13: 27.96$ & $-48: 19: 26.3$ & 19.690 & 0.011 & 1.774 & 0.010 & 1.755 & 1 \\
$5: 13: 34.14$ & $-48: 18: 15.6$ & 19.695 & 0.011 & 2.384 & 0.012 & 2.373 & 1 \\
$5: 13: 33.34$ & $-48: 18: 16.7$ & 19.708 & 0.011 & 2.118 & 0.007 & 2.036 & 1 \\
$5: 13: 30.22$ & $-48: 18: 48.9$ & 19.722 & 0.010 & 1.963 & 0.009 & 1.939 & 1 \\
$5: 13: 34.44$ & $-48: 18: 30.3$ & 19.710 & 0.009 & 1.773 & 0.017 & 1.738 & 1 \\
$5: 13: 30.41$ & $-48: 18: 55.6$ & 19.790 & 0.009 & 2.069 & 0.008 & 2.056 & 5 \\
$5: 13: 37.48$ & $-48: 18: 30.5$ & 19.885 & 0.011 & 1.895 & 0.003 & 1.762 & 6 \\
$5: 13: 35.44$ & $-48: 18: 56.9$ & 20.004 & 0.013 & 2.473 & 0.030 & 2.463 & 1 \\
$5: 13: 31.18$ & $-48: 18: 31.3$ & 20.160 & 0.013 & 1.939 & 0.020 & 1.919 & 5 \\
$5: 13: 34.04$ & $-48: 18: 59.7$ & 20.259 & 0.016 & 1.286 & 0.017 & 1.180 & 2 \\
$5: 13: 28.68$ & $-48: 18: 03.1$ & 21.243 & 0.063 & 1.821 & 0.049 & 1.905 & 7 \\
$5: 13: 32.55$ & $-48: 18: 16.9$ & 20.421 & 0.017 & 1.921 & 0.022 & 1.895 & 2 \\
$5: 13: 36.12$ & $-48: 19: 27.7$ & 20.483 & 0.029 & 2.026 & 0.006 & 2.008 & 1
\end{tabular}




\begin{tabular}{llllllll}
$5: 13: 30.97$ & $-48: 17: 33.9$ & 20.583 & 0.018 & 1.781 & 0.003 & 1.850 & 6 \\
$5: 13: 33.23$ & $-48: 18: 37.1$ & 20.945 & 0.035 & 1.609 & 0.015 & 1.572 & 5 \\
$5: 13: 37.96$ & $-48: 18: 38.9$ & 21.041 & 0.068 & 0.348 & 0.017 & -0.964 & 7 \\
$5: 13: 36.41$ & $-48: 17: 42.5$ & 21.305 & 0.030 & 1.298 & 0.010 & 1.318 & 5 \\
$5: 13: 33.34$ & $-48: 19: 14.3$ & 21.317 & 0.062 & 2.435 & 0.093 & 2.264 & 5 \\
$5: 13: 39.14$ & $-48: 18: 40.8$ & 21.484 & 0.071 & 0.587 & 0.025 & -0.951 & 7 \\
$5: 13: 35.21$ & $-48: 18: 43.4$ & 21.948 & 0.052 & 1.471 & 0.030 & 1.503 & 5 \\
$5: 13: 34.63$ & $-48: 18: 17.8$ & 22.087 & 0.064 & 2.204 & 0.043 & 2.281 & 5 \\
$5: 13: 28.13$ & $-48: 19: 01.4$ & 22.044 & 0.053 & 2.399 & 0.041 & 2.430 & 1 \\
$5: 13: 32.77$ & $-48: 18: 34.6$ & 22.125 & 0.057 & 1.533 & 0.021 & 1.552 & 5 \\
$5: 13: 30.97$ & $-48: 17: 33.9$ & 20.583 & 0.018 & 1.781 & 0.003 & 1.156 & 6 \\
$5: 13: 33.96$ & $-48: 17: 45.8$ & 22.143 & 0.060 & 2.187 & 0.017 & 2.093 & 5 \\
$5: 13: 36.12$ & $-48: 19: 27.7$ & 20.483 & 0.029 & 2.026 & 0.006 & 1.990 & 5 \\
$5: 13: 35.98$ & $-48: 19: 38.7$ & 22.590 & 0.084 & 1.169 & 0.070 & 0.985 & 5 \\
\hline
\end{tabular}

Photometry and morphology for cluster 11

\begin{tabular}{cccccccc}
\hline \hline $\mathrm{RA}(2000)$ & $\operatorname{Dec}(2000)$ & $I$ & $\operatorname{err}_{I}$ & $(V-I)_{3}$ & $\operatorname{err}_{(V-I)_{3}}$ & $(V-I)_{\text {fit }}$ & Morph \\
\hline $10: 45: 52.38$ & $0: 01: 42.9$ & 17.061 & 0.002 & 2.101 & 0.001 & 2.101 & 1 \\
$10: 45: 53.80$ & $0: 01: 12.4$ & 17.909 & 0.003 & 1.517 & 0.002 & 1.552 & 2 \\
$10: 45: 52.50$ & $0: 01: 17.1$ & 19.479 & 0.007 & 2.105 & 0.008 & 2.132 & 1 \\
$10: 45: 50.71$ & $0: 01: 52.1$ & 19.551 & 0.009 & 1.971 & 0.007 & 2.065 & 6 \\
$10: 45: 51.59$ & $0: 01: 45.6$ & 19.631 & 0.006 & 2.029 & 0.009 & 2.082 & 6 \\
$10: 45: 51.42$ & $0: 01: 54.1$ & 19.794 & 0.013 & 1.802 & 0.004 & 1.880 & 1 \\
$10: 45: 51.63$ & $0: 01: 29.6$ & 19.622 & 0.009 & 2.024 & 0.004 & 2.065 & 1 \\
$10: 45: 55.92$ & $0: 01: 06.7$ & 19.649 & 0.006 & 2.044 & 0.009 & 2.086 & 3 \\
$10: 45: 52.78$ & $0: 01: 47.6$ & 19.751 & 0.009 & 2.077 & 0.008 & 2.120 & 6 \\
$10: 45: 48.94$ & $0: 01: 33.9$ & 19.854 & 0.007 & 2.006 & 0.004 & 2.031 & 1 \\
$10: 45: 52.72$ & $0: 01: 51.8$ & 20.455 & 0.022 & 1.990 & 0.006 & 2.054 & 6 \\
$10: 45: 51.82$ & $0: 01: 44.8$ & 20.201 & 0.008 & 1.938 & 0.009 & 2.045 & 6
\end{tabular}




\begin{tabular}{|c|c|c|c|c|c|c|c|}
\hline $10: 45: 51.67$ & 0:02:33.1 & 20.128 & 0.008 & 2.084 & 0.010 & 2.079 & 1 \\
\hline $10: 45: 53.62$ & $0: 01: 48.0$ & 20.164 & 0.013 & 1.968 & 0.027 & 2.104 & 1 \\
\hline $10: 45: 47.80$ & $0: 02: 06.7$ & 20.192 & 0.011 & 1.936 & 0.014 & 1.982 & 1 \\
\hline $10: 45: 50.47$ & 0:01:38.3 & 20.338 & 0.012 & 2.022 & 0.010 & 2.053 & 5 \\
\hline $10: 45: 56.13$ & $0: 00: 58.0$ & 20.393 & 0.011 & 1.992 & 0.010 & 2.006 & 1 \\
\hline $10: 45: 52.58$ & $0: 01: 34.0$ & 20.688 & 0.024 & 1.885 & 0.026 & 1.876 & 6 \\
\hline $10: 45: 50.87$ & $0: 01: 40.1$ & 20.545 & 0.017 & 1.783 & 0.008 & 1.809 & 2 \\
\hline $10: 45: 52.22$ & $0: 01: 30.2$ & 21.421 & 0.051 & 1.750 & 0.127 & 1.861 & 7 \\
\hline $10: 45: 52.68$ & $0: 01: 37.8$ & 20.684 & 0.012 & 2.004 & 0.013 & 2.012 & 7 \\
\hline $10: 45: 51.59$ & $0: 01: 45.6$ & 19.631 & 0.006 & 2.029 & 0.009 & 1.681 & 6 \\
\hline $10: 45: 52.45$ & $0: 01: 38.3$ & 20.946 & 0.009 & 1.994 & 0.005 & 2.061 & 7 \\
\hline $10: 45: 55.19$ & $0: 01: 25.4$ & 20.797 & 0.014 & 1.991 & 0.005 & 2.028 & 5 \\
\hline $10: 45: 50.35$ & $0: 02: 05.8$ & 20.977 & 0.020 & 1.877 & 0.017 & 1.929 & 5 \\
\hline $10: 45: 54.90$ & $0: 01: 05.8$ & 21.004 & 0.021 & 1.899 & 0.018 & 1.927 & 5 \\
\hline $10: 45: 50.13$ & $0: 01: 38.3$ & 21.227 & 0.022 & 1.896 & 0.023 & 1.981 & 5 \\
\hline $10: 45: 55.50$ & $0: 01: 51.2$ & 21.325 & 0.026 & 1.821 & 0.017 & 1.847 & 5 \\
\hline $10: 45: 51.92$ & $0: 01: 58.5$ & 21.945 & 0.043 & 1.901 & 0.036 & 2.119 & 6 \\
\hline $10: 45: 51.43$ & $0: 01: 20.2$ & 21.619 & 0.031 & 1.761 & 0.059 & 1.823 & 5 \\
\hline $10: 45: 50.98$ & $0: 02: 07.4$ & 21.555 & 0.029 & 1.738 & 0.035 & 1.788 & 5 \\
\hline $10: 45: 51.42$ & $0: 01: 54.1$ & 19.794 & 0.013 & 1.802 & 0.004 & 1.287 & 7 \\
\hline $10: 45: 54.18$ & $0: 01: 42.5$ & 21.831 & 0.037 & 1.629 & 0.033 & 1.137 & 5 \\
\hline $10: 45: 55.23$ & $0: 01: 05.2$ & 21.699 & 0.040 & 1.923 & 0.028 & 2.001 & 5 \\
\hline $10: 45: 54.46$ & $0: 00: 50.2$ & 21.731 & 0.032 & 1.917 & 0.015 & 1.936 & 5 \\
\hline $10: 45: 50.66$ & $0: 01: 47.1$ & 22.369 & 0.059 & 1.625 & 0.066 & 1.761 & 6 \\
\hline $10: 45: 52.50$ & $0: 01: 17.1$ & 19.479 & 0.007 & 2.105 & 0.008 & 0.691 & 6 \\
\hline $10: 45: 52.94$ & $0: 01: 08.1$ & 22.317 & 0.088 & 0.740 & 0.071 & 0.852 & 5 \\
\hline $10: 45: 52.69$ & $0: 02: 18.6$ & 22.434 & 0.052 & 1.781 & 0.051 & 1.784 & 5 \\
\hline $10: 45: 53.64$ & $0: 01: 53.2$ & 22.448 & 0.094 & 1.900 & 0.067 & 1.925 & 6 \\
\hline
\end{tabular}


Photometry and morphology for cluster 12

\begin{tabular}{|c|c|c|c|c|c|c|c|}
\hline $\mathrm{RA}(2000)$ & $\operatorname{Dec}(2000)$ & $I$ & $\operatorname{err}_{I}$ & $(V-I)_{3}$ & $\operatorname{err}_{(V-I)_{3}}$ & $(V-I)_{f i t}$ & Morph \\
\hline $10: 46: 58.20$ & $-0: 27: 06.1$ & 19.893 & 0.023 & 1.720 & 0.050 & 1.964 & 2 \\
\hline $10: 47: 02.04$ & $-0: 27: 06.1$ & 19.080 & 0.004 & 2.132 & 0.004 & 2.146 & 1 \\
\hline $10: 47: 02.67$ & $-0: 27: 18.3$ & 19.022 & 0.006 & 1.403 & 0.014 & 1.422 & 2 \\
\hline $10: 47: 02.05$ & $-0: 27: 10.4$ & 19.326 & 0.005 & 2.091 & 0.015 & 2.078 & 6 \\
\hline $10: 47: 03.97$ & $-0: 27: 45.8$ & 19.172 & 0.005 & 2.061 & 0.008 & 2.063 & 2 \\
\hline $10: 47: 03.73$ & $-0: 27: 10.2$ & 19.301 & 0.004 & 1.964 & 0.009 & 1.990 & 1 \\
\hline $10: 47: 01.77$ & $-0: 27: 08.6$ & 19.843 & 0.007 & 2.088 & 0.004 & 2.086 & 1 \\
\hline $10: 47: 02.97$ & $-0: 27: 30.7$ & 19.836 & 0.008 & 2.099 & 0.040 & 2.103 & 2 \\
\hline $10: 47: 01.85$ & $-0: 26: 59.8$ & 20.071 & 0.012 & 2.111 & 0.019 & 2.142 & 2 \\
\hline $10: 47: 00.56$ & $-0: 26: 17.6$ & 20.038 & 0.007 & 1.881 & 0.024 & 1.903 & 2 \\
\hline $10: 46: 59.66$ & $-0: 26: 55.3$ & 20.159 & 0.010 & 1.873 & 0.021 & 1.859 & 2 \\
\hline $10: 47: 02.38$ & $-0: 26: 02.2$ & 20.463 & 0.011 & 2.023 & 0.002 & 1.955 & 6 \\
\hline $10: 47: 02.30$ & $-0: 27: 12.3$ & 20.618 & 0.011 & 1.708 & 0.017 & 1.768 & 2 \\
\hline $10: 47: 04.17$ & $-0: 28: 09.3$ & 20.729 & 0.013 & 1.569 & 0.014 & 1.632 & 2 \\
\hline $10: 47: 01.94$ & $-0: 26: 56.1$ & 20.891 & 0.015 & 2.167 & 0.026 & 2.203 & 2 \\
\hline $10: 47: 01.91$ & $-0: 26: 50.7$ & 20.919 & 0.015 & 2.131 & 0.030 & 2.175 & 6 \\
\hline $10: 47: 04.84$ & $-0: 27: 38.8$ & 21.060 & 0.018 & 2.411 & 0.031 & 2.496 & 2 \\
\hline $10: 47: 00.28$ & $-0: 26: 30.8$ & 21.142 & 0.017 & 1.805 & 0.050 & 1.863 & 5 \\
\hline $10: 47: 06.12$ & $-0: 27: 45.8$ & 21.252 & 0.018 & 1.643 & 0.039 & 1.636 & 2 \\
\hline $10: 47: 01.85$ & $-0: 26: 35.1$ & 21.446 & 0.022 & 1.979 & 0.011 & 1.993 & 5 \\
\hline $10: 47: 01.49$ & $-0: 27: 14.2$ & 21.907 & 0.035 & 1.978 & 0.102 & 1.985 & 5 \\
\hline $10: 47: 02.52$ & $-0: 27: 31.6$ & 21.779 & 0.043 & 1.483 & 0.039 & 1.522 & 5 \\
\hline $10: 47: 04.59$ & $-0: 27: 43.5$ & 23.044 & 0.099 & 2.615 & 0.157 & 2.079 & 7 \\
\hline $10: 47: 03.61$ & $-0: 27: 21.6$ & 22.110 & 0.044 & 1.812 & 0.055 & 1.834 & 5 \\
\hline $10: 47: 00.98$ & $-0: 27: 17.8$ & 22.276 & 0.072 & 0.901 & 0.191 & 0.892 & 5 \\
\hline $10: 47: 03.74$ & $-0: 26: 15.8$ & 22.302 & 0.046 & 1.556 & 0.098 & 1.577 & 5 \\
\hline $10: 47: 01.65$ & $-0: 26: 48.8$ & 22.257 & 0.043 & 2.095 & 0.041 & 2.148 & 5 \\
\hline
\end{tabular}




\begin{tabular}{llllllll}
$10: 47: 04.31$ & $-0: 27: 32.1$ & 22.389 & 0.088 & 0.913 & 0.091 & 1.014 & 5 \\
\hline
\end{tabular}

\section{Photometry and morphology for cluster 15}

\begin{tabular}{|c|c|c|c|c|c|c|c|}
\hline $\mathrm{RA}(2000)$ & $\operatorname{Dec}(2000)$ & $I$ & $\operatorname{err}_{I}$ & $(V-I)_{3}$ & $\operatorname{err}(V-I)_{3}$ & $(V-I)_{f i t}$ & Morph \\
\hline $13: 47: 30.65$ & $-11: 45: 09.5$ & 17.082 & 0.002 & 1.695 & 0.004 & 1.742 & 1 \\
\hline $13: 47: 31.87$ & $-11: 45: 11.4$ & 17.610 & 0.002 & 2.202 & 0.005 & 2.228 & 1 \\
\hline 13:47:30.05 & $-11: 45: 41.9$ & 18.764 & 0.005 & 2.186 & 0.007 & 2.220 & 1 \\
\hline $13: 47: 27.27$ & $-11: 45: 43.4$ & 18.841 & 0.004 & 2.220 & 0.007 & 2.251 & 1 \\
\hline $13: 47: 29.46$ & $-11: 46: 02.7$ & 18.901 & 0.005 & 2.207 & 0.002 & 2.226 & 1 \\
\hline $13: 47: 27.88$ & $-11: 45: 53.8$ & 19.245 & 0.004 & 1.998 & 0.011 & 1.990 & 6 \\
\hline $13: 47: 32.18$ & $-11: 45: 18.2$ & 19.401 & 0.007 & 2.084 & 0.003 & 2.091 & 2 \\
\hline $13: 47: 32.46$ & $-11: 45: 04.2$ & 19.374 & 0.006 & 2.130 & 0.009 & 2.154 & 1 \\
\hline $13: 47: 28.05$ & $-11: 45: 51.6$ & 19.470 & 0.005 & 2.102 & 0.015 & 2.123 & 6 \\
\hline $13: 47: 31.89$ & $-11: 45: 06.9$ & 19.767 & 0.004 & 2.130 & 0.002 & 2.098 & 6 \\
\hline $13: 47: 33.29$ & $-11: 44: 21.5$ & 19.660 & 0.006 & 2.045 & 0.007 & 2.066 & 1 \\
\hline $13: 47: 30.47$ & $-11: 44: 49.6$ & 20.664 & 0.029 & 2.087 & 0.054 & 2.151 & 1 \\
\hline $13: 47: 28.08$ & $-11: 44: 18.8$ & 19.786 & 0.008 & 2.069 & 0.007 & 2.112 & 2 \\
\hline $13: 47: 29.09$ & $-11: 46: 00.5$ & 19.772 & 0.007 & 1.940 & 0.005 & 2.000 & 1 \\
\hline $13: 47: 31.83$ & $-11: 45: 23.2$ & 20.554 & 0.025 & 2.026 & 0.055 & 2.072 & 6 \\
\hline $13: 47: 30.68$ & $-11: 44: 58.5$ & 20.058 & 0.012 & 2.047 & 0.030 & 2.074 & 6 \\
\hline $13: 47: 30.01$ & $-11: 45: 19.7$ & 19.966 & 0.008 & 2.135 & 0.009 & 2.156 & 1 \\
\hline $13: 47: 32.79$ & $-11: 46: 14.8$ & 19.990 & 0.008 & 1.974 & 0.013 & 2.035 & 1 \\
\hline $13: 47: 31.24$ & $-11: 45: 30.3$ & 20.647 & 0.023 & 2.132 & 0.028 & 2.120 & 6 \\
\hline $13: 47: 34.80$ & $-11: 45: 01.5$ & 20.023 & 0.007 & 2.116 & 0.022 & 2.159 & 1 \\
\hline $13: 47: 27.43$ & $-11: 45: 56.6$ & 20.037 & 0.008 & 2.113 & 0.009 & 2.300 & 1 \\
\hline $13: 47: 33.55$ & $-11: 44: 33.7$ & 20.056 & 0.008 & 2.087 & 0.013 & 2.117 & 1 \\
\hline $13: 47: 26.91$ & $-11: 44: 24.4$ & 20.061 & 0.007 & 2.157 & 0.010 & 2.150 & 5 \\
\hline $13: 47: 30.85$ & $-11: 45: 26.6$ & 20.077 & 0.015 & 2.125 & 0.027 & 2.158 & 6 \\
\hline $13: 47: 31.47$ & $-11: 45: 04.9$ & 20.123 & 0.012 & 2.103 & 0.033 & 2.133 & 2 \\
\hline
\end{tabular}




\begin{tabular}{|c|c|c|c|c|c|c|}
\hline $13: 47: 26.10$ & $-11: 45: 21.4$ & 20.114 & 0.007 & 2.130 & 0.010 & 2.153 \\
\hline $13: 47: 32.18$ & $-11: 45: 55.5$ & 20.144 & 0.008 & 2.092 & 0.013 & 2.144 \\
\hline $13: 47: 30.71$ & $-11: 45: 40.5$ & 20.203 & 0.012 & 2.062 & 0.015 & 2.077 \\
\hline $13: 47: 32.55$ & $-11: 44: 56.9$ & 20.520 & 0.020 & 2.093 & 0.022 & 2.170 \\
\hline $13: 47: 34.05$ & $-11: 44: 28.5$ & 20.207 & 0.009 & 2.106 & 0.024 & 2.073 \\
\hline $13: 47: 29.90$ & $-11: 44: 56.8$ & 20.325 & 0.013 & 2.152 & 0.023 & 2.164 \\
\hline $13: 47: 29.71$ & $-11: 44: 01.0$ & 20.250 & 0.011 & 2.059 & 0.013 & 2.041 \\
\hline $13: 47: 28.02$ & $-11: 44: 54.7$ & 20.309 & 0.009 & 2.039 & 0.032 & 2.089 \\
\hline $13: 47: 31.71$ & $-11: 45: 01.2$ & 20.322 & 0.018 & 2.022 & 0.045 & 2.038 \\
\hline $13: 47: 29.60$ & $-11: 44: 50.1$ & 20.346 & 0.011 & 2.063 & 0.022 & 2.072 \\
\hline 13:47:30.61 & $-11: 45: 53.1$ & 20.427 & 0.012 & 2.061 & 0.016 & 2.075 \\
\hline 13:47:33.19 & $-11: 45: 40.7$ & 20.365 & 0.009 & 2.028 & 0.018 & 2.055 \\
\hline $13: 47: 31.55$ & $-11: 45: 17.3$ & 20.692 & 0.009 & 2.098 & 0.028 & 2.020 \\
\hline $13: 47: 35.08$ & $-11: 45: 13.4$ & 20.521 & 0.012 & 1.780 & 0.022 & 1.825 \\
\hline $13: 47: 32.76$ & $-11: 45: 13.1$ & 20.568 & 0.016 & 2.188 & 0.030 & 2.179 \\
\hline 13:47:30.31 & $-11: 45: 36.9$ & 20.507 & 0.013 & 2.244 & 0.024 & 2.247 \\
\hline $13: 47: 31.59$ & $-11: 44: 52.8$ & 20.870 & 0.021 & 2.217 & 0.049 & 2.175 \\
\hline $13: 47: 29.45$ & $-11: 45: 01.8$ & 20.627 & 0.013 & 2.083 & 0.030 & 2.156 \\
\hline $13: 47: 28.72$ & $-11: 45: 51.9$ & 20.682 & 0.015 & 2.057 & 0.054 & 2.073 \\
\hline 13:47:32.37 & $-11: 45: 34.4$ & 20.683 & 0.011 & 2.119 & 0.013 & 2.096 \\
\hline $13: 47: 31.03$ & $-11: 45: 41.6$ & 20.718 & 0.013 & 2.053 & 0.018 & 2.086 \\
\hline $13: 47: 32.37$ & $-11: 44: 50.1$ & 21.169 & 0.029 & 2.042 & 0.035 & 2.089 \\
\hline $13: 47: 29.33$ & $-11: 45: 54.7$ & 22.219 & 0.077 & 1.698 & 0.151 & 1.899 \\
\hline 13:47:30.07 & $-11: 45: 01.5$ & 20.965 & 0.028 & 2.253 & 0.091 & 2.298 \\
\hline 13:47:31.51 & $-11: 45: 58.1$ & 20.772 & 0.013 & 2.070 & 0.010 & 2.077 \\
\hline $13: 47: 28.47$ & $-11: 45: 52.5$ & 21.064 & 0.024 & 2.237 & 0.062 & 2.235 \\
\hline 13:47:30.39 & $-11: 46: 09.6$ & 20.866 & 0.013 & 2.041 & 0.024 & 1.974 \\
\hline $13: 47: 27.92$ & $-11: 45: 08.7$ & 21.059 & 0.023 & 0.782 & 0.051 & 1.303 \\
\hline $13: 47: 31.55$ & $-11: 45: 17.3$ & 20.692 & 0.009 & 2.098 & 0.028 & 1.810 \\
\hline 13:47:31.15 & $-11: 45: 47.7$ & 20.883 & 0.014 & 2.220 & 0.021 & 2.210 \\
\hline
\end{tabular}




\begin{tabular}{|c|c|c|c|c|c|c|}
\hline $13: 47: 31.89$ & $-11: 46: 16.2$ & 21.025 & 0.015 & 2.075 & 0.020 & 2.125 \\
\hline $13: 47: 33.79$ & $-11: 45: 45.9$ & 21.083 & 0.016 & 2.062 & 0.019 & 2.110 \\
\hline $13: 47: 32.94$ & $-11: 45: 15.1$ & 21.240 & 0.023 & 2.212 & 0.123 & 2.225 \\
\hline $13: 47: 30.34$ & $-11: 44: 40.1$ & 21.098 & 0.022 & 2.025 & 0.024 & 2.066 \\
\hline 13:47:30.39 & $-11: 46: 09.6$ & 20.866 & 0.013 & 2.041 & 0.024 & 2.096 \\
\hline $13: 47: 31.25$ & $-11: 45: 27.6$ & 21.416 & 0.028 & 2.065 & 0.021 & 2.055 \\
\hline $13: 47: 30.01$ & $-11: 44: 34.8$ & 21.223 & 0.019 & 1.411 & 0.033 & 1.412 \\
\hline $13: 47: 28.67$ & $-11: 45: 17.3$ & 21.261 & 0.019 & 1.898 & 0.069 & 1.921 \\
\hline $13: 47: 34.14$ & $-11: 44: 52.6$ & 21.255 & 0.021 & 1.795 & 0.020 & 2.027 \\
\hline $13: 47: 31.51$ & $-11: 45: 10.8$ & 21.377 & 0.010 & 2.092 & 0.010 & 2.000 \\
\hline $13: 47: 30.01$ & $-11: 45: 19.7$ & 19.966 & 0.008 & 2.135 & 0.009 & 2.112 \\
\hline $13: 47: 32.00$ & $-11: 45: 21.6$ & 21.547 & 0.036 & 2.170 & 0.063 & 2.371 \\
\hline $13: 47: 29.49$ & $-11: 45: 26.3$ & 21.325 & 0.030 & 1.953 & 0.018 & 1.973 \\
\hline $13: 47: 30.05$ & $-11: 46: 04.5$ & 21.334 & 0.021 & 2.126 & 0.063 & 2.216 \\
\hline $13: 47: 30.92$ & $-11: 46: 06.1$ & 21.362 & 0.019 & 2.001 & 0.031 & 2.037 \\
\hline $13: 47: 31.51$ & $-11: 45: 10.8$ & 21.377 & 0.010 & 2.092 & 0.010 & 2.009 \\
\hline $13: 47: 28.51$ & $-11: 44: 18.3$ & 21.399 & 0.019 & 2.093 & 0.049 & 2.096 \\
\hline $13: 47: 26.91$ & $-11: 44: 24.4$ & 20.061 & 0.007 & 2.157 & 0.010 & 2.151 \\
\hline $13: 47: 30.62$ & $-11: 45: 47.3$ & 22.260 & 0.050 & 2.164 & 0.079 & 2.099 \\
\hline $13: 47: 30.67$ & $-11: 44: 33.9$ & 21.496 & 0.027 & 2.059 & 0.033 & 2.071 \\
\hline $13: 47: 29.18$ & $-11: 44: 41.6$ & 21.534 & 0.022 & 2.128 & 0.008 & 2.173 \\
\hline $13: 47: 34.53$ & $-11: 45: 49.7$ & 21.526 & 0.022 & 1.390 & 0.050 & 1.401 \\
\hline $13: 47: 31.55$ & $-11: 45: 17.3$ & 20.692 & 0.009 & 2.098 & 0.028 & 2.067 \\
\hline $13: 47: 27.88$ & $-11: 45: 53.8$ & 19.245 & 0.004 & 1.998 & 0.011 & 3.424 \\
\hline $13: 47: 34.98$ & $-11: 45: 05.3$ & 21.524 & 0.022 & 1.508 & 0.077 & 1.547 \\
\hline $13: 47: 32.80$ & $-11: 45: 06.9$ & 22.209 & 0.058 & 2.024 & 0.071 & 2.235 \\
\hline $13: 47: 32.55$ & $-11: 44: 56.9$ & 20.520 & 0.020 & 2.093 & 0.022 & 2.194 \\
\hline $13: 47: 28.12$ & $-11: 45: 29.9$ & 21.847 & 0.028 & 1.948 & 0.034 & 1.997 \\
\hline $13: 47: 29.18$ & $-11: 46: 11.7$ & 21.721 & 0.035 & 1.169 & 0.024 & 1.144 \\
\hline $13: 47: 33.86$ & $-11: 45: 07.4$ & 21.758 & 0.025 & 1.916 & 0.028 & 1.922 \\
\hline
\end{tabular}




\begin{tabular}{llllllll}
$13: 47: 29.25$ & $-11: 44: 13.6$ & 21.914 & 0.033 & 2.054 & 0.061 & 2.053 & 5 \\
$13: 47: 26.84$ & $-11: 45: 46.0$ & 21.873 & 0.031 & 2.038 & 0.041 & 2.032 & 5 \\
$13: 47: 28.40$ & $-11: 45: 21.0$ & 22.099 & 0.034 & 1.944 & 0.088 & 0.530 & 6 \\
$13: 47: 29.10$ & $-11: 44: 57.9$ & 22.008 & 0.036 & 2.015 & 0.115 & 2.130 & 5 \\
$13: 47: 29.92$ & $-11: 45: 17.6$ & 21.386 & 0.015 & 2.106 & 0.052 & 2.257 & 6 \\
$13: 47: 32.99$ & $-11: 44: 42.7$ & 22.526 & 0.049 & 2.271 & 0.088 & 2.172 & 5 \\
$13: 47: 28.05$ & $-11: 45: 51.6$ & 19.470 & 0.005 & 2.102 & 0.015 & 1.688 & 7 \\
$13: 47: 32.55$ & $-11: 44: 56.9$ & 20.520 & 0.020 & 2.093 & 0.022 & 2.431 & 6 \\
$13: 47: 31.86$ & $-11: 44: 27.1$ & 22.178 & 0.052 & 1.782 & 0.008 & 1.849 & 5 \\
$13: 47: 32.37$ & $-11: 45: 34.4$ & 20.683 & 0.011 & 2.119 & 0.013 & 2.081 & 6 \\
$13: 47: 30.59$ & $-11: 45: 24.2$ & 22.313 & 0.045 & 2.106 & 0.110 & 2.161 & 7 \\
$13: 47: 34.49$ & $-11: 45: 01.8$ & 22.381 & 0.047 & 1.829 & 0.037 & 2.000 & 6 \\
$13: 47: 31.47$ & $-11: 44: 18.7$ & 22.433 & 0.058 & 1.315 & 0.053 & 1.225 & 2 \\
$13: 47: 28.20$ & $-11: 45: 20.0$ & 22.583 & 0.052 & 1.968 & 0.125 & 2.052 & 5 \\
$13: 47: 33.28$ & $-11: 45: 48.7$ & 22.473 & 0.045 & 1.985 & 0.016 & 2.002 & 5 \\
$13: 47: 31.97$ & $-11: 44: 36.8$ & 22.474 & 0.081 & 2.383 & 0.099 & 2.167 & 5 \\
\hline
\end{tabular}

Photometry and morphology for cluster 19

\begin{tabular}{cccccccc}
\hline \hline $\operatorname{RA}(2000)$ & $\operatorname{Dec}(2000)$ & $I$ & $\operatorname{err}_{I}$ & $(V-I)_{3}$ & $\operatorname{err}_{(V-I)_{3}}$ & $(V-I)_{f i t}$ & Morph \\
\hline $23: 59: 16.72$ & $-32: 17: 18.9$ & 18.598 & 0.004 & 2.060 & 0.016 & 2.139 & 1 \\
$23: 59: 19.58$ & $-32: 18: 08.7$ & 19.375 & 0.006 & 2.105 & 0.018 & 2.175 & 1 \\
$23: 59: 14.05$ & $-32: 17: 13.4$ & 19.400 & 0.006 & 1.836 & 0.009 & 1.909 & 1 \\
$23: 59: 17.48$ & $-32: 16: 52.8$ & 19.563 & 0.007 & 1.666 & 0.017 & 1.738 & 6 \\
$23: 59: 17.45$ & $-32: 17: 23.1$ & 19.915 & 0.009 & 1.982 & 0.028 & 2.070 & 1 \\
$23: 59: 17.03$ & $-32: 17: 41.4$ & 19.846 & 0.010 & 2.132 & 0.007 & 2.214 & 1 \\
$23: 59: 17.27$ & $-32: 17: 12.5$ & 20.133 & 0.014 & 1.999 & 0.012 & 2.111 & 2 \\
$23: 59: 17.26$ & $-32: 17: 26.3$ & 20.234 & 0.011 & 2.202 & 0.016 & 2.226 & 6 \\
$23: 59: 13.86$ & $-32: 18: 01.4$ & 20.209 & 0.009 & 1.239 & 0.024 & 1.304 & 5 \\
$23: 59: 18.97$ & $-32: 16: 26.0$ & 20.238 & 0.011 & 1.763 & 0.024 & 1.830 & 6
\end{tabular}




\begin{tabular}{|c|c|c|c|c|c|c|c|}
\hline $23: 59: 15.25$ & $-32: 17: 33.1$ & 20.503 & 0.015 & 1.732 & 0.025 & 1.823 & 5 \\
\hline 23:59:13.11 & $-32: 16: 56.2$ & 20.607 & 0.016 & 2.342 & 0.025 & 2.503 & 5 \\
\hline $23: 59: 16.70$ & $-32: 16: 51.0$ & 20.582 & 0.015 & 1.800 & 0.015 & 1.898 & 2 \\
\hline 23:59:18.66 & $-32: 17: 19.2$ & 20.688 & 0.015 & 1.880 & 0.028 & 1.974 & 1 \\
\hline 23:59:15.18 & $-32: 17: 22.7$ & 20.859 & 0.024 & 1.369 & 0.026 & 1.443 & 5 \\
\hline $23: 59: 20.09$ & $-32: 16: 38.2$ & 20.900 & 0.020 & 1.722 & 0.008 & 1.851 & 5 \\
\hline $23: 59: 15.73$ & $-32: 17: 40.5$ & 20.965 & 0.020 & 1.818 & 0.037 & 1.888 & 5 \\
\hline $23: 59: 20.18$ & $-32: 18: 09.1$ & 20.990 & 0.019 & 2.056 & 0.042 & 2.162 & 5 \\
\hline $23: 59: 18.65$ & $-32: 17: 48.0$ & 21.049 & 0.019 & 1.987 & 0.043 & 2.150 & 5 \\
\hline $23: 59: 17.18$ & $-32: 17: 20.8$ & 21.070 & 0.014 & 1.998 & 0.028 & 1.997 & 6 \\
\hline $23: 59: 21.25$ & $-32: 17: 17.0$ & 21.212 & 0.024 & 1.404 & 0.011 & 1.499 & 2 \\
\hline 23:59:17.86 & $-32: 17: 48.1$ & 21.284 & 0.027 & 1.801 & 0.077 & 1.850 & 6 \\
\hline $23: 59: 16.78$ & $-32: 17: 11.4$ & 21.363 & 0.032 & 1.840 & 0.004 & 1.725 & 6 \\
\hline $23: 59: 14.86$ & $-32: 17: 00.2$ & 21.411 & 0.032 & 1.890 & 0.009 & 2.015 & 5 \\
\hline 23:59:17.64 & $-32: 17: 43.6$ & 21.630 & 0.034 & 2.023 & 0.023 & 2.216 & 2 \\
\hline 23:59:21.77 & $-32: 17: 08.5$ & 21.701 & 0.031 & 1.313 & 0.043 & 1.353 & 5 \\
\hline $23: 59: 21.63$ & $-32: 16: 40.2$ & 21.825 & 0.053 & 0.927 & 0.064 & 1.332 & 7 \\
\hline 23:59:17.93 & $-32: 17: 21.6$ & 22.168 & 0.066 & 0.633 & 0.055 & 1.414 & 6 \\
\hline $23: 59: 16.72$ & $-32: 16: 57.4$ & 22.228 & 0.045 & 1.469 & 0.038 & 2.067 & 6 \\
\hline $23: 59: 14.86$ & $-32: 17: 00.2$ & 21.411 & 0.032 & 1.890 & 0.009 & 1.777 & 6 \\
\hline $23: 59: 16.72$ & $-32: 17: 18.9$ & 18.598 & 0.004 & 2.060 & 0.016 & 3.562 & 7 \\
\hline 23:59:16.72 & $-32: 17: 18.9$ & 18.598 & 0.004 & 2.060 & 0.016 & 1.970 & 7 \\
\hline 23:59:13.91 & $-32: 17: 39.4$ & 22.299 & 0.068 & 1.467 & 0.031 & 1.585 & 5 \\
\hline 23:59:17.48 & $-32: 17: 41.2$ & 22.677 & 0.074 & 1.584 & 0.110 & 1.835 & 7 \\
\hline
\end{tabular}




\section{References}

Akritas, M. and Bershady, M. 1996. Astrophys. J. 470, 706.

Alongi, M., Bertelli, G., Bressan, A., Chiosi, C., Fagotto, F., Greggio, L., and Nasi, E. 1993. Astron. Astrophys. Suppl. 97, 851.

Aragón-Salamanca, A., Ellis, R., Couch, W., and Carter, D. 1993. Mon. Not. R. Astron. Soc. 262, 764.

Arimoto, N. and Yoshii, Y. 1987. Astron. Astrophys. 173, 23. (AY87).

Barger, A., Aragón-Salamanca, A., Smail, I., Ellis, R., Couch, W., Dressler, A., Oemler, A., and Poggianti, B. 1998. Astrophys. J. 501, 522.

Barrientos, L. F., Schade, D., and López-Cruz, O. 1996. Astrophys. J. Lett. 460, L89.

Baugh, C. M., Cole, S., and Frenk, C. S. 1996. Mon. Not. R. Astron. Soc. 283, 1361. Baugh, C. M., Cole, S., Frenk, C.S., and Benson, A. J. 1998. In To appear in Star Formation in Early-type galaxies, P. Carral and J. Cepa (eds.), Asp. Conf. Ser.

Beers, T. C., Flynn, K., and Gebhardt, K. 1990. Astron. J. 100, 32.

Bender, R., Ziegler, B., and Bruzual, G. 1996. Astrophys. J. 463, 51.

Bertin, E. and Arnouts, S. 1996. Astron. Astrophys. Suppl. 117, 393.

Bessell, M. S. and Brett, J. M. 1988. Publ. Astron. Soc. Pac. 100, 1134.

Bessell, M. S., Brett, J. M., Scholz, M., and Wood, P. R. 1989. Astron. Astrophys. Suppl. 77, 1.

Binney, J. and Merrifield, M. 1998. Galactic Astronomy. Princeton Series in Astrophysics, New Jersey: Princeton University Press.

Birkes, D. and Dodge, Y. 1993. Alternative Methods of Regression. New York: John Wiley \& sons, Inc.

Böhm-Vitense, E. 1972. Astron. Astrophys. 17, 335.

Bower, R., Lucey, J., and Ellis, R. 1992a. Mon. Not. R. Astron. Soc. 254, 601.

Bower, R., Lucey, J., and Ellis, R. 1992b. Mon. Not. R. Astron. Soc. 254, 589.

Bower, R., Böhringer, H., Briel, U., Ellis, R., Castander, F., and Couch, W. 1994. Mon. Not. R. Astron. Soc. 268, 345. 
Bower, R., Castander, F., Couch, W., Ellis, R., and Böhringer, H. 1997. Mon. Not. R. Astron. Soc. $291,353$.

Bressan, A., Fagotto, F., Bertelli, G., and Chiosi, C. 1993. Astron. Astrophys. Suppl. $100,647$.

Bressan, A., Chiosi, C., and Fagotto, F. 1994. Astrophys. J. Suppl. 94, 63. (BCF94).

Bruzual, G. and Charlot, S. 1993. Astrophys. J. 405, 538.

Bruzual, G. and Charlot, S. 1996. (BC96), in preparation.

Burstein, D. and Heiles, C. 1982. Astron. J. 887, 1165.

Buser, R. and Kurucz, R. L. 1978. Astron. Astrophys. 70, 555.

Buzzoni, A. 1995. Astrophys. J. Suppl. 98, 69.

Carlberg, R., Yee, H., Ellingson, E., Abraham, R., Gravel, P., Morris, S., and Pritchet, C. 1996. Astrophys. J. 462, 32.

Carrasco, L., Recillas-Cruz, E., García-Barreto, A., Cruz-González, I., and Serrano, A. 1991. Publ. Astron. Soc. Pac. 103, 987.

Casali, M. and Hawarden, T. 1992. JCMT-UKIRT Newsletter 4, 33.

Charlot, S., Worthey, G., and Bressan, A. 1996. Astrophys. J. 457, 625.

Coleman, G., Wu, C., and Weedman, D. 1980. Astrophys. J. Suppl. 43, 393.

Couch, W. and Shanks, T. 1985. Mon. Not. R. Astron. Soc. 213, 215.

Couch, W., Ellis, R., Malin, D., and MacLaren, I. 1991. Mon. Not. R. Astron. Soc. 249, 606.

de Propis, R., Eisenhardt, P., Standford, S., and Dickinson, M. 1998. Astrophys. J. Lett. 503, L45.

Dickinson, M. 1995. Astron. Soc. Pac. Conf. Series 86, 283.

Djorgovski, G. and Davis, M. 1987. Astrophys. J. 313, 59.

Dressler, A., Lynden-Bell, D., Burstein, D., Davies, R., Faber, S., Terlevich, R., and Wegner, G. 1987. Astrophys. J. 313, 42.

Ellis, R., Smail, I., Dressler, A., Couch, W., Oemler, A., Butcher, H., and Sharples, R. 1997. Astrophys. J. 483, 582.

Elson, D., van P. Dokkum, Franx, M., Illingworth, G., and Fabricant, D. 1997. Astrophys. J. Lett. 478, L13. 
Fagotto, F., Bressan, A., Bertelli, G., and Chiosi, C. 1994a. Astron. Astrophys. Suppl. 100, 647.

Fagotto, F., Bressan, A., Bertelli, G., and Chiosi, C. 1994b. Astron. Astrophys. Suppl. 104, 647.

Fagotto, F., Bressan, A., Bertelli, G., and Chiosi, C. 1994c. Astron. Astrophys. Suppl. 105, 39.

Feigelson, E. and Babu, G. 1992. Astrophys. J. 397, 55.

Fioc, M. and Rocca-Volmerange, B. 1997. Astron. Astrophys. 362, 950. (FR97).

Flower, P. J. 1977. Astron. Astrophys. 54, 31.

Fluks, M. A., Plez, B., Thé, P. S., de Winter, D., Westerlund, B. E., and Steenman, H. C. 1994. Astron. Astrophys. 285, 751.

Fukugita, M., Shimasaku, K., and Ichikawa, T. 1995. Publ. Astron. Soc. Pac. 107, 945.

Gladders, M., López-Cruz, O., Yee, H., and Kodama, T. 1998. Astrophys. J. 501, 571.

Green, E. M., Demarque, P., and King, C. R. 1987. The Revised Yale Isochrones and Luminosity Functions, Yale University Observatory.

Guiderdoni, B. and Rocca-Volmerange, B. 1987. Astron. Astrophys. 186, 1.

Gunn, J. E. and Stryker, L. L. 1983. Astrophys. J. Suppl. 52, 121.

Gunn, J., Hoessel, J., and Oke, B. 1986. Astrophys. J. 306, 30.

Hamuy, M., Phillips, M., Suntzeff, N., Schommer, B., Maza, J., and Aviles, R. 1996. Astron. J. 112, 239.

Im, M., Griffiths, R., Ratnatunga, K., and Sarajedini, V. 1996. Astrophys. J. Lett. $461,79$.

Johnson, H. 1966. Annu. Rev. Astron. Astrophys. 4, 193.

Joyce, R. 1992. Astron. Soc. Pac. Conf. Series 23, 258.

Kauffmann, G. and Charlot, S. 1998. Mon. Not. R. Astron. Soc. 294, 705.

Kauffmann, G. 1996. Mon. Not. R. Astron. Soc. 281, 487.

Kodama, T. and Arimoto, N. 1997. Astron. Astrophys. 320, 41.

Kormendy, J. 1977. Astrophys. J. 218, 333. 
Kuntschner, H. and Davies, R. 1998. Mon. Not. R. Astron. Soc. 295, L29.

Kurucz, R. L. 1992. In The Stellar Populations of Galaxies, B. Barbuy, A. Renzini, Kluwer, and Dordrecht (eds.), IAU Symp. 149, page 225.

Lancon, A. and Rocca-Volmerange, B. 1992. Astron. Astrophys. Suppl. 96, 593.

Landolt, A. 1992. Astron. J. 104, 340.

Lilly, S., Cowie, L., and Gardner, J. 1991. Astrophys. J. 369, 79.

Lilly, S., Tresse, L., Hammer, F., Crampton, D., and Fevre, O. Le. 1995. Astrophys. J. 455, 108.

Lilly, S. 1987. Mon. Not. R. Astron. Soc. 229, 573.

López-Cruz, O. 1997. PhD thesis, University of Toronto.

Marzke, R., da Costa, L. N., Pellegrini, P., Willmer, C., and Geller, M. 1998. Astrophys. J. 503, 617.

Mihalas, D. and Binney, J. 1981. Galactic Astronomy. New York: W. H. Freeman and Company, 2nd edition.

Oemler, G. 1992. In Clusters and Superclusters of Galaxies, A. C. Fabian (ed.), page 29 .

Oke, J. B. and Gunn, J. 1983. Astrophys. J. 266, 713.

Oke, J. B., Gunn, J., and Hoessel, J. 1996. Astron. J. 111, 29.

Pahre, M., Djorgovski, G., and de Carvalho, R. 1996. Astrophys. J. Lett. 456, 79.

Persson, S. E. 1987. unpublished.

Postman, M., Lubin, L., and Oke, B. 1998. Astron. J. 116, 583.

Press, W., Teukolsky, S., Vetterling, W., and Flannery, B. 1992. Numerical Recipes. Cambridge: Cambridge University Press.

Rakos, K. and Schombert, J. 1995. Astrophys. J. 439, 47.

Recillas-Cruz, E., Carrasco, L., Serrano, A., and Cruz-González, I. 1990. Astron. Astrophys. 229, 64.

Ridgway, S. T., Joyce, R. R., White, N. M., and Wing, R. F. 1980. Astrophys. J. 235, 126.

Rocca-Volmerange, B. and Guiderdoni, B. 1988. Astron. Astrophys. Suppl. 75, 93.

Roche, N., Ratnatunga, K., Griffiths, R., Im, M., and Naim, A. 1998. Mon. Not. R. 
Astron. Soc. 293, 157.

Sandage, A. and Visvanathan, N. 1978. Astrophys. J. 225, 742.

Sawicki, M., Lin, H., and Yee, H. 1997. Astron. J. 113, 1.

Schade, D., Barrientos, L. F., and López-Cruz, O. 1997. Astrophys. J. Lett. 477, L17.

Schechter, P. 1976. Astrophys. J. 203, 297.

Schindler, S., Guzzo, L., Ebeling, H., Böhringer, H., Chincarini, G., Collins, C., de Grandi, S., Neumann, D., Briel, U., Shaver, P., and Vettolani, G. 1995. Astron. Astrophys. 299, L9.

Schindler, S., Hattori, M., Neumann, D., and Böhringer, H. 1997. Astron. Astrophys. 317, 646.

Standford, S., Eisenhardt, P., and Dickinson, M. 1998. Astrophys. J. 492, 461.

Stanford, S., Eisenhardt, P., and Dickinson, M. 1995. Astrophys. J. 450, 512.

Steidel, C. and Hamilton, D. 1993. Astron. J. 105, 2017.

Stetson, P. 1987. Publ. Astron. Soc. Pac. 99, 191.

Straizys, V. and Sviderskiene, Z. 1972. Bull Vilnius Obs. 35, 3.

Tantalo, R., Chiosi, C., and Fagotto, F. 1996. Astron. Astrophys. 311, 361. (TCF96).

Tantalo, R. 1998. private communication.

Terndrup, D. M., Frogel, J. A., and Withford, A. E. 1990a. Astrophys. J. 357, 453.

Terndrup, D. M., Frogel, J. A., and Withford, A. E. 1990b. Astrophys. J. 378, 742. Tyson, J. A. 1986. J. Opt. Soc. Am. 3, 2131.

van Dokkum, P., Franx, M., Kelston, D., and Illingworth, G. 1998. Astrophys. J. Lett. 504, L17.

VandenBerg, D. A. and Bell, R. A. 1985. Astrophys. J. Suppl. 58, 561.

VandenBerg, D. A. and Laskarides, P. G. 1987. Astrophys. J. Suppl. 64, 103.

VandenBerg, D. A. 1985. Astrophys. J. Suppl. 58, 711.

Wamsteker, W. 1981. Astron. Astrophys. 97, 329.

Whitmore, B. 1995. Photometry with the WFPC2. Space Telescope Science Institute. (available on their WWW pages).

Wilson, G., Smail, I., Ellis, R., and Couch, W. 1997. Mon. Not. R. Astron. Soc. 284, 
915.

Worthey, G. 1994. Astrophys. J. Suppl. 95, 107. (W94). 